\title{
Investigating the build-up of precedence effect using reflection masking
}

\author{
Hartcher-O'Brien, Jessica; Buchholz, Jörg
}

Published in:

Journal of the Acoustical Society of America

Publication date:

2006

Document Version

Publisher's PDF, also known as Version of record

Link back to DTU Orbit

Citation (APA):

Hartcher-O'Brien, J., \& Buchholz, J. (2006). Investigating the build-up of precedence effect using reflection masking. In Journal of the Acoustical Society of America (Vol. 120). Acoustical Society of America.

\section{General rights}

Copyright and moral rights for the publications made accessible in the public portal are retained by the authors and/or other copyright owners and it is a condition of accessing publications that users recognise and abide by the legal requirements associated with these rights.

- Users may download and print one copy of any publication from the public portal for the purpose of private study or research.

- You may not further distribute the material or use it for any profit-making activity or commercial gain

- You may freely distribute the URL identifying the publication in the public portal

If you believe that this document breaches copyright please contact us providing details, and we will remove access to the work immediately and investigate your claim. 


\title{
Session 2aAA
}

\section{Architectural Acoustics: Acoustics of Large Indoor and Outdoor Performance Spaces I}

\author{
Daniel R. Raichel, Cochair \\ 2727 Moore Ln., Fort Collins, CO 80526 \\ Toshiki Hanyu, Cochair \\ Nihon Univ., Narashinodai, Funabashi, Chiba 274-8501, Japan
}

Chair's Introduction-7:30

Invited Papers

$7: 35$

2aAA1. Differences in dealing with the acoustics of large outdoor and indoor listening spaces. Daniel R. Raichel (Eilar Assoc. and the Graudate Ctr., CUNY, 2727 Moore Ln., Fort Collins, CO 80526, draichel@ comcast.net)

It was perhaps fortuitous that Wallace Clement Sabine's first consulting assignment from the Harvard Board of Overseers, the execution of which proved to be seminal in the history of architectural acoustics, entailed an indoor facility. The problems of dealing with outdoor performance areas are considerably greater than those encountered in indoor spaces. Indoor spaces provide surface reflections and absorption of sound, both of which can be manipulated through proper design to yield the desired results with respect to reverberation time and sound dispersion. In the case of outdoor performing areas, there are no reflective surfaces (except in the cases of certain amphitheaters), and the need for sound amplification is often necessitated. A reflective bandshell is generally required to direct the program material toward the audience. The lack of wall reflections tends to result in a lack of musical sonority that is usually present in a well-designed indoor auditorium. A number of specific problems with interior and outdoor spaces and modern approaches toward resolving acoustical problems of large listening areas will be discussed.

$7: 55$

2aAA2. Prediction of reverberation by stochastic processes in a forest: Creation of reverberation in an outdoor performance space. Toshiki Hanyu and Katsuaki Sekiguchi (Nihon Univ., Narashinodai, Funabashi, 274-8501, Chiba, Japan, hanyu@arch.jcn.nihon-u.ac.jp)

A forest is an outdoor space. For that reason, reverberation formulas of indoor spaces cannot be used when predicting forest reverberation. As a matter of fact, because the level decay curve of the reverberant sound produced in a forest is nonlinear, the concept of reverberation time is inapplicable. We clarified that reverberation of a forest is expressible as a Wiener process (Brownian motion), which is a stochastic process, and we derived the formula of reverberation of a forest based on that fact. In this study, Monte Carlo simulation was also carried out. Results clarified the following. (1) The energy decay curve of the reverberation sound of a forest becomes a straight line on the logarithmic scale of time and energy; its line gradient is -2. (2) At a point far from a sound source, the reverberant sound energy increases first and begins to decrease after some period. (3) When the mean-free path is short, the sound level of reverberation becomes high. Furthermore, we devised some methods of generating reverberation in outdoor space using the forest reverberation mechanism. For example, a proposed reverberation wall can produce reverberations using only one wall incorporating sound scatterers.

\section{8:15}

2aAA3. Large outdoor performance venues: Acoustical lessons learned. William J. Cavanaugh and K. Anthony Hoover (327F Boston Post Rd., Sudbury, MA 01776)

The Tweeter Center for the Performing Arts (formerly Great Woods) in Mansfield, MA, off Interstate 495, midway between Boston and Providence, accommodates 19900 patrons and offers 75 to 85 performances each year between 15 May and 15 October. The Pittsburgh Symphony Orchestra inaugurated Tweeter Center's first concert season in June 1986, and The Center hosted the PSO as its summer home for three additional seasons. The Center has hosted performances from solo comedians to symphonic orchestras and rock bands, featuring such diverse acts as the Boston Pops, James Taylor, Phish, and The Kinks. The acoustical lessons learned over two decades of operation are described. The Center has served as a model for the design and operation of facilities in the 20000 -capacity range, including the often difficult issues of controlling excessive amplified sound transmission to residential neighbors, as well as assuring good listening conditions for patrons and performers alike. 
2aAA4. Design of public address system for emergency evacuation in a tunnel. Sakae Yokoyama, Hideki Tachibana (Chiba Inst. of Technol., 2-17-1 Tsudanuma, Narashino-city, Chiba, 275-0016, Japan, sakae@iis.u-tokyo.ac.jp), Shinichi Sakamoto (Univ. of Tokyo, Meguro-ku, Tokyo, 153-8505, Japan), and Seiya Tazawa (Metropolitan Expressway Co. Ltd., Chiyoda-ku, Tokyo, 100-8930, Japan)

As a means to improve speech intelligibility of a public address PA system for emergency evacuation announcement in a tunnel, the authors have been investigating the application of the successive time-delay technique and have performed the experiments in an actual tunnel. In the experiments, the effect of the technique has been examined by comparing the difference of speech intelligibility between the conditions with/without time delay. For the design of the PA system in a reverberant tunnel, four kinds of psychoacoustical experiments on speech intelligibility were performed in an anechoic room using the six-channel recording/reproduction technique and the following results have been obtained: (1) the successive time-delay technique is very effective to improve the speech intelligibility in a tunnel, (2) the system is considerably robust against the error in the delay time, (3) the speech intelligibility improves with the increase of the speech-rate but it should be suitable for such a tense situation as fire emergency, and (4) in order to further improve the speech intelligibility, it is necessary to control the reverberation in the tunnel by any sound absorption treatment. In this paper, the outline of the field experiment and the results of subjective experiments are introduced.

\section{8:55}

2aAA5. The Jay Pritzker Music Pavilion at Millennium Park, part 1: Design and construction. Richard Talaske (TALASKE, 105 N. Oak Park Ave., Oak Park, IL 60301)

The acoustic and audio system design for the Pritzker Pavilion represents a leap forward in the outdoor listening experience. The venue offers superior sound experiences to audiences of 11000 using audio reinforcement and acoustic enhancement systems to support all types of musical performances, particularly for the resident Grant Park Music Festival orchestra. With reliance on the audio systems to project and envelop the audience in sound, the design of the stage enclosure was able to focus on the acoustic quality for the ensemble, a rare opportunity in outdoor facilities. The stage enclosure design includes variable acoustic devices and has an emphasis on intraensemble communication. This presentation will discuss the design of the stage enclosure, the trellis-supported audio systems, and the ways in which the facility is used by resident and visiting musical performers.

\section{9:15}

2aAA6. The Jay Pritzker Music Pavilion at Millennium Park, part 2: Research and testing. Gregory Miller, Richard Talaske, Byron Harrison, Evelyn Way (TALASKE, 105 N. Oak Park Ave., Oak Park, IL 60301), Lily Wang, David Bradley, and Craig Johnson (Univ. of Nebraska-Lincoln, Omaha, NE 68182-0681)

The design of the Pritzker Pavilion includes a number of innovative acoustic and audio elements. Two of these elements, the resilient orchestra riser system and the acoustic enhancement system, will be discussed in this presentation. Research previously presented to the ASA [Hoffman et.al., 141st meeting of the ASA) was used in the design of the orchestra riser system at the Pritzker Pavilion. This presentation discusses the field tests that were conducted to assess vibration transfer across the surface of the riser system. Comparison is made to previous research and anticipated results. Subjective evaluations by orchestra members will also be discussed. The acoustic enhancement system is a specialized audio system, separate from the main reinforcement audio system, based on LARES digital signal processing and a distributed loudspeaker approach. Different settings have been developed over time to provide varying decay signatures that are employed on a music-program-dependent basis. Impulse response measurements were taken of unamplified sound, the reinforcement system, and the combination of the reinforcement system with the enhancement system. Testing was conducted with faculty and students from the University of Nebraska.

\section{9:35}

2aAA7. Acoustical design of the "Tenseishinbikai Hakko Shinden" Shrine in Shiga. Masayuki Ikegami (Dept. of Acoust Eng., Tech. Res. Inst., Obayashi Corp., 4-640 Simokiyoto, Kiyose City, Tokyo 208-8558, Japan)

A shrine called the "Tenseishinbikai Hakko Shinden" was designed and constructed. It covers a large area and has seating for 3690 people. It is used mainly for religious ceremonies and occasionally for concerts. This report outlines its acoustical design and noise control measures. The acoustics design specifically addressed reverberation control appropriate for its use, in addition to echo-nuisance prevention. The flat fan shape of the building often engenders insufficient initial sound reflection and generates echoes. To avoid these problems, in consultation with the designer, an irregular reflective surface was used for the side walls, a rib-shaped acoustic absorption surface, and a folding-screen shaped diffusion surface was used for the rear wall. In addition to conventional machine room noise control, particular attention had to be devoted to glass walls, rain noise, etc. For the glass walls, the effects on the outside (sound generated in the event room) and the effects on the inside (outdoor traffic noise) had to be controlled. To control rain noise, the sound generated by rain on the metal roof had to be predicted; a suitable roof material was selected.

\section{9:55}

2aAA8. Sound around the New England Patriots' stadia. Anthony K. Hoover, Andrew Carballeira, and Matthew Moore (Cavanaugh Tocci Assoc., Inc., 327 F Boston Post Rd., Sudbury, MA 01776)

The New England Patriots wanted to build a new football stadium in Foxboro, MA, adjacent to the old one which would be demolished after the new one was opened. The Environmental Impact Statement (EIS) indicated that one benefit would be a 3.34-dB reduction of stadium noise at the nearest town line because the distance would increase by $980 \mathrm{ft}$. A procedure to test the resultant noise reduction was developed with keen interest from the Massachusetts Department of Environmental Protection. Touring rock 
concert sound systems would output high levels of pink noise, twice during sound-checks in the old stadium and then twice in the new stadium; resultant levels would be measured at several locations around the old stadium and then again around the new stadium. This paper will summarize the results of the measurements and will discuss interesting aspects of a test procedure designed by committee, including the importance of declining to be responsible for certain expectations of an EIS.

10:15-10:35 Break

\section{$10: 35$}

2aAA9. Sound system design for acoustically challenging spaces. Akira Mochimaru and Soichiro Hayashi (Bose K.K., 28-3-Maruyama-Cho, Shibuya YT Bldg., Shibuya-Ku, Tokyo, Japan)

Sound reinforcement (SR) systems take a critical part in controlling acoustic performance in large venues. Loudspeaker system design including consideration of room acoustics becomes crucial in providing audio information with good intelligibility and natural tonal balance throughout the audience area. There are many elements that can spoil acoustic performance of the sound system in large spaces. Relatively long reverberation time, number of speaker clusters with distances in between, size of each speaker cluster that creates unexpected phase cancellations and so on. Neither the single speaker product nor sound system design based on experiences can provide a perfect solution for these kinds of spaces. On the other hand, an advanced acoustic simulation system including consideration of relative phase between sound sources with capability of listening to the simulated results is now available. By using this technology with academically proven accuracy, speaker array design methodology called bass-array was produced. Bass-array allows the sound system designer to control radiation directivity characteristics of low frequency. Design of custom line-array to suit room configuration of the space is another deliverable of the technology. This technology in association with bass-array or line-array has been successfully applied for providing appropriate acoustic solutions in a number of acoustically difficult spaces.

\section{$10: 55$}

2aAA10. Recording in an outdoor performance space. Pamela Harght (Berklee College of Music, 150 Massachusetts Ave., Boston, MA 02115)

For both audio engineers and musicians, dealing with indoor and outdoor spaces poses several types of problems for recording and performance purposes. While recording indoors poses many problems, most of them (pertaining to room design, ventilation, placement of musicians) are controllable. However, dealing with outdoor spaces creates many issues entailing uncontrollable situations, specifically those entailing the environment. During the summer of 2006, the author spent 8 weeks at the Tanglewood Music Center in Lenox, MA on an audio engineering fellowship. Tanglewood is the summer home for the Boston Symphony Orchestra; it has both an outdoor performance space and a concert hall that opens for additional lawn seating. This paper discusses and compares the many issues of recording classical music outdoors as well as indoors, based upon experience, research, and interviews with musicians and members of the audio department.

\section{1:15}

2aAA11. Acoustics for the one percenter. Scott Pfeiffer (Threshold Acoust. LLC, 954 West Washington, 5th Fl., Chicago, IL 60607)

GMC adopted the one percenter from outlaw bikers who took pride in identifying themselves as the $1 \%$ of bikers that were making a bad name for the rest of the $99 \%$ that were law abiding. GMC and the outlaw bikers had the right idea. We all like to identify ourselves with the few, the proud, those that appreciate the special, the esoteric, the pursuit of greatness. Large venue projects present a special challenge to reaching these goals as the issues of getting 5000 or more people in and out begins to drive the design, natural acoustics become challenging or impossible, and the distribution of audio and video begins to depend on delay clusters and secondary distribution, which all begin as compromises. Doubt enters as the question is raised of how many patrons will notice. It is in these moments when finding the design that serves the one percenter makes all of the difference. Special examples of one percenters will be presented as a retrospective of large venues honoring the most successful projects in this type.

\section{1:35}

2aAA12. Improving sound qualities at the seats under deep balcony overhangs. Wei-hwa Chiang, Wei Lin, and Kun-tai Wu (Dept. of Architecture, Natl. Taiwan Univ. of Sci. and Technol., \#43, Sec.4, Keelung Rd., Taipei, 106, Taiwan, edchiang1224@hotmail.com)

Source-receiver distances in a large hall could be reduced by increasing the balcony overhangs, which, however, may cause insufficient acoustical energy for the under-balcony seats. Besides, the reduction in the sense of reverberation cannot be easily mitigated. In this research various design strategies, including utilizing the technique of a flying balcony, were examined in a 1550 -seat hall with deep balcony overhangs. Both BEM simulation and scale model measurements were performed. Optimizing the profile of the under-balcony space and utilizing ceiling and side reflectors near the platform were effective in enhancing early reflection. This is especially meaningful for high-frequency string sounds that radiate against the ceiling. Opening the rear part of the balcony that formed a partially flying balcony could compensate late reflections. Preliminary scale modeling results showed that approximately $15 \%$ increase in early decay time can be achieved with carefully designed ceiling geometry when the opening area was only $7 \%$ of the overhang area. 


\title{
Session 2aAB
}

\section{Animal Bioacoustics: Fish Bioacoustics: A Tribute to Arthur Myrberg I}

\author{
Arthur N. Popper, Cochair \\ Univ. of Maryland, Dept. of Biology, College Park, MD 20742 \\ Richard R. Fay, Cochair \\ Loyola Univ. Chicago, Parmly Hearing Inst., 6525 N. Sheridan Rd., Chicago, IL 60626
}

Chair's Introduction-8:00

Invited Papers

8:05

2aAB1. A tribute to Art Myrberg. John H. Stadler (Natl. Marine Fisheries Service, 801 Lilly Rd. NE, Lacey, WA 98503) and Samuel L. Gruber (Univ. of Miami, Miami, FL 33149)

Arthur A. Myrberg, Jr. passed away in April, 2005, and will be remembered by many as a pioneer in the fields of fish bioacoustics and behavior. His distinguished career spanned more than 40 years, and saw him become a world-recognized expert in these fields, publishing more than 90 peer-reviewed articles, some of which are considered seminal. During more than 35 years as a professor of Marine Science at the Rosenstiel School of Marine and Atmospheric Sciences, University of Miami, he was a well-respected and admired scientist, teacher, lecturer, mentor, and advisor to 24 graduate students. This presentation will trace Art's life from his days as a youth in Lulenecks, Illinois, through his college career and his work with Nobel laureate Konrad Lorenz at the Max Planck Institute, Seiweisen, Germany, culminating in his long career at the University of Miami.

8:25

2aAB2. A history of fish bioacoustic studies. Arthur N. Popper (Dept. of Biol., Univ. of Maryland, College Park, MD 20742, apopper@umd.edu), Richard R. Fay (Loyola Univ. of Chicago, Chicago, IL 60626), and William N. Tavolga (Mote Marine Lab., Sarasota, FL 34236)

Interest in fish sound production and hearing dates back to the writings of Aristotle (384-322 BCE) and Pliny the Elder (23-79 $\mathrm{CE})$, both of whom noted not only that fish make sounds, but also speculated that fish must be able to hear despite their not having apparent ears. The fact that fish could hear was first demonstrated with the work of G. H. Parker in 1903 and later by Karl von Frisch. Studies on sound production were sporadic in the early part of the 20th century and often based on anecdotes until technology derived from antisubmarine warfare became available after World War II. This equipment enabled investigators to listen to fish and correlate sounds with behavior. It was not until the early 1960s that intensive investigations started on fish hearing. This paper will review the history of the study of fish bioacoustics and discuss the contributions of a large number of investigators including those of Arthur Myrberg. Indeed, Art's research stands as a landmark in the field, as well as a fine example of true scholarship. Furthermore, his charismatic presentations of his work were instrumental in stimulating the investigations of other scientists.

8:45

2aAB3. Art Myrberg's contribution to the study of hearing in fishes. Richard R. Fay (Parmly Hearing Inst. and Dept. of Psych., Loyola Univ. Chicago, 6525 N. Sheridan Rd., Chicago, IL 60626, rfay@luc.edu)

Art Myrberg was trained to study behavior from an ethological perspective. When it came to studying the hearing of the vocal and communicating damselfish species of the genus Eupomacentrus, he surprisingly decided to use conditioning methods in combination with the best technology then known to produce an excellently designed animal psychoacoustic (laboratory) study that remains today, 26 years later, an exemplary model for students in this field [A. A. Myrberg and J. Y. Spires, J. Comp. Physiol. 140, 135-144 (1980)]. First, Myrberg well understood that sensitivity to acoustic particle motion was possible for these species, so he produced or simulated sound in the far, free field using a long tube with sound absorbers at the opposite end, and measured both sound pressure and acoustic particle motion. He documented that Eupomacentrus was sensitive to particle motion at $100 \mathrm{~Hz}$, but was sound-pressure-sensitive at higher frequencies. He also understood that ambient noise was an important issue in hearing studies, and he did all that he could to measure and reduce it. The result was a "V"-shaped audiogram with greatest sensitivity at $500 \mathrm{~Hz}$, and almost no interspecific variation. We can have great confidence in these audiograms today as valid measurements of hearing ability.

\section{9:05}

2aAB4. Art Myrberg's contributions to fish acoustic communication. Michael L. Fine (Dept. Biol., Virginia Commonwealth Univ., Richmond, VA 23284-2012)

Arthur Myrberg was an ichthyologist and ethologist who trained with the Nobel laureate Konrad Lorenz. Art's work exceeded the boundaries of communication (a signal produced by an animal for its own benefit) to include related phenomena such as acoustic attraction and repulsion of sharks, effects of noise, and the creation of the term interception to describe a communication signal picked 
up (e.g., intercepted) by another receiver for its own benefit. His work included a wide range of species (sharks, surgeonfish, damselfish, cichlids, gobies, and even invertebrates and marine mammals). He worked at sea and utilized diving for observation and experimental manipulation and then set up lab colonies for further observation and experimentation. What is perhaps his signal contribution to acoustic communication followed this paradigm in an investigation in four related Eupomacentrus species that produce similar chirps during a courtship dip. By playbacks of natural and altered sounds he and his students separated the subtle cues used by these fishes to permit intraspecific communication. This rather dry abstract fails to capture Art's love of science, his ability to make outstanding presentations, and my value of his friendship.

\section{9:25}

2aAB5. Recent advances in fish acoustic behavior. Timothy Tricas (Dept. of Zoology and Hawaii Inst. of Marine Biol., Univ. of Hawaii at Manoa, Honolulu, HI 96822, tricas@ hawaii.edu)

Sounds produced by fish provide important signals or cues in many behavioral contexts. Mechanisms of sound production are diverse and best described in fishes with specialized anatomical structures, but remain tentative for many species. The coproduction of low-frequency hydrodynamic and acoustic stimuli during behavioral interactions can excite the inner ear, lateral line, or both to provide complex acoustico-lateralis information. Recent behavior studies show that acoustic signals function in behavioral displays of aggression, species recognition, courtship, mate attraction, and spawning; in some cases it can function as a single modality signal. Sound stimuli often overlap with the hearing band of highest sensitivity (100-1000 Hz for most species), but in some species these do not match. Much data now exist on the diversity of sounds produced by fishes and environmental acoustic cues, but more evaluation is needed within the context of the animals natural environment. Examples include acoustic cues for recruitment of pelagic fish larvae to reefs, use of ultrasound to avoid predators, and infrasound stimuli. Comparative work on both sound production and sensory reception in fishes can be assessed in relation to their ecology to better understand the evolution of fish acoustic behavior.

\section{9:45}

2aAB6. Lateral line research: Recent advances and new opportunities. Sheryl Coombs (Dept. of Biological Sci., Bowling Green State Univ., Bowling Green, OH 43403) and Horst Bleckmann (Univ. of Bonn, 53115 Bonn, Germany)

Computational fluid dynamics, digital particle imaging velocimetry, and engineered micro flow sensors, along with emerging collaborations between neurobiologists and engineers, have opened up new vistas for lateral line research in recent years. Engineering advances have increased our ability to measure the spatiotemporal characteristics of biologically significant, more complex flow signals and how these signals are sensed and processed by the lateral line. Following in the rich tradition of Art Myrberg's work on sound communication in fishes, studies on hydrodynamic signal production and use by fish during interactive social behaviors are now feasible. In addition to these new opportunities, several recent studies have enriched the lateral line landscape. The lateral line sensing and encoding of vortex trails shed by swimming fish or obstacles in a stream have been investigated, along with associated behaviors (e.g., vortex trail following and obstacle entrainment). Blind Mexican cavefish have been shown to form cognitive maps of their surroundings using lateral-line based, hydrodynamic cues. Finally, the responses of goldfish auditory and lateral line systems to the same stimulus source have been documented to reveal how these two closely related systems might work in concert to extract complementary but different types of information from nearby hydroacoustic sources.

\section{0:05-10:20 Break}

\section{Contributed Papers}

\section{0:20}

2aAB7. Year-round changes of ambient noise in freshwater habitats and temperature effects on hearing in fishes. Sonja Amoser and Friedrich Ladich (Dept. of Neurobiology and Behavioral Sci., Univ. of Vienna, Althanstr. 14, 1090 Vienna, Austria)

Fish get crucial information for their survival from their acoustic environment. Therefore, changes in ambient noise as well as temperature over the year potentially impose great challenges for fishes, especially in freshwater habitats in temperate climates. Noise levels vary more in stagnant habitats such as lakes, backwaters, and streams than in large rivers. The spectral profiles change in stagnant habitats due to biotic noise sources in summer (e.g., stridulating insects), whereas in small streams spectral composition differs due to small changes in water volume, e.g., after snow melt. To investigate possible seasonal effects on fishes, we studied the hearing capabilities of a hearing specialist (Common carp) and a hearing nonspecialist (European perch) at two different temperatures $\left(12{ }^{\circ} \mathrm{C}\right.$ and $\left.22{ }^{\circ} \mathrm{C}\right)$ using the AEP recording technique. In the carp the hearing sensitivity decreased up to $12 \mathrm{~dB}$ (mean decrease: $4 \mathrm{~dB}$ ) at $12{ }^{\circ} \mathrm{C}$, whereas no such change was observed in the perch. Latencies of auditory evoked potentials became longer by approximately $0.8 \mathrm{~ms}$ at lower temperatures in the carp. The results indicate that auditory sensitivity in hearing specialists is affected by changes in temperature as well as ambient noise throughout the year. [Work supported by FWF.]

\section{0:35}

2aAB8. The influence of ambient temperature and thermal acclimation on hearing in an eurythermal hearing specialized fish. Lidia E. Wysocki and Arthur N. Popper (Dept. of Biol., Univ. of Maryland, College Park, MD 20742, 1wysocki@umd.edu)

Many fish species live in habitats whose water temperature underlies seasonal and diurnal fluctuations. Being ectothermic animals, their body temperature depends on the temperature of the ambient water. Since the speed of all metabolic processes depends on body temperature, the ambient temperature might affect various physiological processes including all sensory systems. However, the maintenance of sensory functions in a changing environment can be crucial for an animal's survival. Several fish species strongly rely on their auditory abilities for acoustic orientation, communication, detecting predators and prey, and finding mates. Channel catfish (Ictalurus punctatus) was chosen as a model for an eurytherm species with a relatively high hearing sensitivity and bandwidth to investigate the influence of ambient temperature and thermal acclimation on the auditory system. Hearing sensitivity was measured at different water temperatures to which the animals were either unacclimated or acclimated by recording auditory-evoked potentials. The ambient water temperature significantly influenced hearing thresholds and the shape of the auditoryevoked potentials, especially at higher frequencies. Hearing sensitivity remained decreased at low water temperatures even after an acclimation period of one month, indicating that certain physical limits on sensory processes cannot be compensated for by thermal acclimation. 
these studies indicate that the fundamental role in efferent modulation of afferent input is likely conserved in both nonmammalian vertebrates and mammals. [Work supported by NIH/NIDCD Grant R01DC003275.]

2aAB9. Investigating hearing in sturgeon: Getting closer to the origin of hearing in vertebrates. Michaela Meyer, Arthur N. Popper (Dept. of Biol., Univ. of Maryland, College Park, MD 20742), and Richard R. Fay (Loyola Univ. of Chicago, Chicago, IL 60626)

It has been hypothesized that hearing arose early in vertebrate evolution to use sound to glean information about the environment, including the presence of predators and prey. The importance of sound to sense the acoustic scene is especially true underwater where the visual horizon is limited. To create an acoustic scene from sound, an animal needs to localize and identify sound sources. We hypothesize that the sound localization capabilities of primitive bony fishes such as sturgeon are as capable as those of modern bony fishes such as goldfish and, perhaps, many terrestrial vertebrates. We present results from experiments investigating the neural mechanisms of directional hearing and frequency analysis in the auditory periphery of the sturgeon. Extracellular single units were recorded from the eighth nerve during stimulation with a three-axis shaker system that subjects the fish ear to linear accelerations in known directions at various frequencies. This system enables the characterization of both spatial and frequency tuning of the afferents innervating the ear. The results show that similar neural mechanisms for encoding sound direction and frequency exist in the periphery of modern and primitive bony fish, thereby supporting the hypothesis that basic auditory functions arose very early in vertebrate history.

\section{1:05}

2aAB10. Recent advances in the fish auditory efferent system. Zhongmin Lu (Dept. of Biol., Univ. of Miami, 1301 Memorial Dr., Coral Gables, FL 33146, zlu@miami.edu) and Seth Tomchik (Univ. of Miami, Miami, FL 33101)

The octavolateral efferent neurons in fish project axons to innervate the ear and lateral line organs. However, the function of the octavolateral efferent system is not well understood. This review focuses on recent progress in structure and function of octavolateral efferent system in a teleost, the sleeper goby (Dormitator latifrons). The sleeper goby has a single octavolateral efferent nucleus that is composed of about 19 large, densely packed somata in the medulla. Most efferent neurons have bilateral dendrites and single axons innervating ipsilateral peripheral octavolateral organs. The efferent neurons have distinctive characteristics of frequency, temporal, directional, and dynamic responses, which differ from those of otolithic organ afferents. How efferent stimulation affects afferent response is investigated by electrically stimulating efferent somata and recording compound action potentials from afferents innervating the saccule, the main auditory organ in this species. There are different efferent modulatory effects on afferent response, depending on the level of background noise. Efferent activation inhibits afferent response in quiet conditions but can enhance signal detection in noisy environments. Results of

\section{$11: 20$} hearing. Charlotte W. Kotas, Peter H. Rogers, and Minami Yoda (Mech.

The fish inner ear contains a dense, bony otolith that oscillates in response to acoustic excitation. This relative motion between the otolith and its surrounding fluid generates both steady streaming and unsteady flows in the fluid which in turn displaces the hair cells on the macular membrane. Fish "hear" by interpreting these hair cell displacements. However, most of the hair cells are located near a groove along the otolith (the sulcus), making it likely that the irregular geometric features of the otolith will affect how fish hear by influencing the motions of these hair cells. To understand these acoustically induced flows, magnified otolith models were constructed based upon three-dimensional computer tomography scans of actual otoliths. The model otoliths were sinusoidally oscillated so that steady streaming and unsteady flows could be experimentally studied using particle-image velocimetry. The effect of geometric features like the sulcus was studied near the model surface at various oscillation frequencies, $\omega$, and amplitudes, $s$. Results will be presented for Reynolds number $\operatorname{Re} \equiv \omega L^{2} / \nu \approx 1-10^{2}$ and amplitude ratios $\epsilon \equiv s / L=0.05--0.2$, where $L$ is a representative otolith length scale and $\nu$ is the kinematic viscosity of the fluid. [Work supported by ONR.]

\section{1:35}

2aAB12. Particle motion perception in cephalopods. Kenzo Kaifu, Susumu Segawa (Tokyo Univ. of Marine Sci. and Technol. 4-5-7, Kounan, Minato-ku, Tokyo, Japan), and Tomonari Akamatsu (Fisheries Res. Agency, Kamisu, Ibaraki, Japan)

In the 1980s, there still had been the argument whether cephalopods could detect sound stimulus or not. The ability of sound detecting (hearing ability or an ability to detect vibration) in cephalopods was proved around 1990, however, there have been few studies about sound detection in cephalopods afterwards. Based on morphological and behavioral studies, it seems that cephalopods do not perceive sound pressure. Provided that compressional waves do not stimulate the nervous systems of the animals, they do response to sound waves due to water particle movement. In this study, the ability to detect sound waves in cephalopods was investigated from the standpoint of water particle movement perception. An object would be well vibrated by sound waves when the length of the object is much smaller relative to the wavelength; consequently, when an animal detects water particle movement, the smaller the animal is, the higher frequency and weaker stimulus it would detect.
2aAB11. Flows near model otoliths and their implications for fish Eng. Dept., Georgia Inst. of Technol., Atlanta, GA 30332-0405) 


\title{
Session 2aAOa
}

\section{Acoustical Oceanography and Underwater Acoustics: Acoustic Scattering by Aquatic Organisms III}

\author{
Kenneth G. Foote, Cochair \\ Woods Hole Oceanographic Inst., Woods Hole, MA 02543 \\ Masahiko Furusawa, Cochair \\ Tokyo Univ. of Marine Science and Technology, 4-5-7 Konan, Minato, Tokyo 108-8477, Japan
}

Chair's Introduction-8:00

Invited Papers

8:05

2aAOa1. Fish population and behavior revealed by instantaneous continental-shelf scale imaging. Nicholas C. Makris (Dept. of Mech. Eng., MIT, 77 Massachusetts Ave., Cambridge MA 02139), Purnima Ratilal (Dept. of Elec. and Comput. Eng., Northeastern Univ.), Deanelle T. Symonds, Srinivasan Jagannathan, Sunwoong Lee (Dept. of Mech. Eng., MIT, Cambridge, MA), and Redwood W. Nero (Naval Res. Lab., Stennis Space Ctr., MS)

Fish populations in continental shelf environments are instantaneously imaged over thousands of square kilometers and continuously monitored by a remote sensing technique in which the ocean acts as an acoustic waveguide [Science 311, 660-663 (2006)]. The technique, ocean acoustic waveguide remote sensing (OAWRS), has revealed the instantaneous horizontal structural characteristics and volatile short-term behavior of very large fish shoals, containing tens of millions of fish and stretching for many kilometers. The former follows a fractal or power-law spectral process, indicative of structural similarity at all scales. The latter features compressional waves of fish population density that travel roughly an order of magnitude faster than fish can swim. Here we present an overview of OAWRS imagery documenting fish activity over a 2-week period in the Spring of 2003 at the edge of the continental shelf, roughly $200 \mathrm{~km}$ south of Long Island, NY. Until now, continental shelf environments have been monitored with highly localized line-transect methods from slow-moving research vessels. These methods significantly undersample fish populations in time and space, leaving an incomplete and ambiguous record of abundance and behavior.

8:25

2aAOa2. Acoustical imaging of nonbladdered animals using underwater acoustic camera. Kohji Iida, Rika Takahashi, Tohru Mukai (Fac. Fish., Hokkaido Univ., 3-1-1 Minato, Hakodate, Hokkaido, 0418611 Japan, iidacs@ fish.hokudai.ac.jp), Yong Tang (Dalian Fish. Univ., Dalian 116023 China), and Masanori Sato (Honda Electron. Co., Ltd., Toyohashi, Aichi, 4413193 Japan)

An underwater acoustic camera using 3.5-MHz ultrasonic waves enclosed in a pressure-resistant case was constructed to observe underwater marine animals. This enabled the measurement of the size, shape, and behavior of living marine animals including internal organs by B-mode images in the detection range up to $240 \mathrm{~cm}$. Observations were conducted for captive animals in a water tank and for natural animals in a field. The captive animals, including fish, squid, and jellyfish, were observed, and a three-dimensional internal structure of animals was reconstructed using multiple acoustical images. The most important contributors of acoustic scattering were the vertebra for nonbladdered fish, and the liver and reproductive organs for invertebrate animals. In a field experiment, the shape, size, and swimming behavior of wild animals were observed. The possibilities and limitations of the underwater acoustic camera for fishery applications were discussed. [Work supported by JSPS Grant-In-Aid for Scientific Research.]

\section{$8: 45$}

2aAOa3. Simultaneous observations of zooplankton distribution and density using net sampling, a laser-optical plankton counter, and acoustics. Tohru Mukai, Yasuzumi Fujimori, Jun-ichiro Kishi, and Kohji Iida (Faculty of Fisheries Sci., Hokkaido Univ., 3-1-1 Minato, Hakodate, Hokkaido 0418611, Japan)

Conventional plankton sampling methods are based on net tows, which are often poorly suited to capturing spatiotemporal variability. Various automatic plankton samplers based on optical principles have been developed recently. The laser-optical plankton counter (LOPC) was developed as the next generation OPC. In addition, acoustic technology has been used extensively to survey zooplankton distribution and abundance. This study compared the density and size spectra determined using net contents, LOPC data, and acoustic data. Surveys were conducted for 3 years around Hokkaido, Japan. The sound-scattering layer (SSL) was recorded on the echogram of an echosounder. Biological sampling was conducted using a $1-\mathrm{m}^{2}$ frame-type mid-water trawl (FMT; mesh size $1.5 \mathrm{~mm}$ ) and a NORPAC net (mesh size 500 microns). An LOPC was mounted inside the mouth of these nets. Net sampling was carried out at dusk, when the SSL moves toward the surface (ca. 10-35 m). Acoustic data were derived using a Simrad EK60 echosounder operating at 38, 120, and $200 \mathrm{kHz}$ simultaneously. A comparison of the use of acoustic data, LOPC data, and net contents to assess zooplankton distribution and density will be presented and discussed. [Work supported by JSPS Grants-In-Aid for Scientific Research.] 


\section{Contributed Papers}

2aAOa4. Riding the wave: Scale-dependent descriptions of walleye pollock distributions. Julian M. Burgos, John K. Horne (School of Aquatic and Fishery Sci., Univ. of Washington, 1122 NE Boat St., Seattle, WA 98105, jmburgos@u.washington.edu), and John K. Horne (Natl. Marine Fisheries Service, NOAA, Seattle, WA 98115)

Wavelet analysis was used to describe scale-dependent distributional variability of walleye pollock (Theragra chalcogramma) in the eastern Bering Sea shelf between 2000 and 2002. Area backscattering strength $(\mathrm{ABC})$, a measure of acoustic density, was calculated at 20-m horizontal intervals in five echo-integration trawl surveys. The maximum overlap discrete wavelet transform was used to partition the $\mathrm{ABC}$ variance into scales ranging from 0.04 to $10.24 \mathrm{~km}$. Wavelet variance was estimated for each transect after denoising with phase plane thresholding. Wavelet spectra can be characterized by large variances at small scales (less than 0.64 $\mathrm{km}$ ), decreasing when scale increased to $1.28 \mathrm{~km}$, and increasing variance at larger scales. The fraction of the total variance assigned to small scales was negatively correlated with the total acoustic density. This is attributed to the aggregative behavior of walleye pollock. Peaks in wavelet spectra, indicating the presence of coherent features (i.e., schools or shoals), were observed in $48 \%$ of the transects at scales ranging from 1.28 to $10.24 \mathrm{~km}$. Peaks at larger scales were more common in winter transects, when walleye pollock form large spawning aggregations.

\section{9:20}

2aAOa5. An overview of the theoretical framework for measuring fish swimming speed by a sector-scanning sonar. Akira Hamano (Natl. Fisheries Univ., 2-7-1, Nagata-honmachi, Shimonoseki, Japan 759-6595, ahamano@fish-u.ac.jp), Jennifer Miksis-Olds, Christopher Jakubiak, and Ernest Bernard (Univ. of Massachusetts Dartmouth, New Bedford, MA 02744-1221)

Two identical high-frequency sector-scanning sonars, mounted on a stationary acoustical-optical platform (AOP), were used to study the swimming speed of fishes. The first phase of this study consisted of a motion calibration performed in an acoustic test tank using a standard target sphere (WC $38.1 \mathrm{~mm}$ ). The sonars are a $200-\mathrm{kHz}$ sector-scanning system employing an 8-element array to receive echo signals over a $90^{\circ}$ arc. An overview of the theoretical framework for the motion calibration of the sector-scanning sonar is presented. The second phase of the study related the calibration data to the observed movement of fish. The twodimensional swimming tracks were identified from the digitized sonar data recorded while the AOP was deployed on the seafloor. These tracks were compared with the movement of the target sphere to make estimates of fish swimming speed. Optical data, when available, were used to confirm the results of acoustic data. The limitations and precision of swimming speed measurements using this system are discussed. The application of this methodology to studies of fine-scale fish behavior has the potential to make previously intractable questions possible. [Work supported by NOAA.]

\section{9:35}

2aAOa6. Depth- and size-dependent rhythms of animal emergence in an estuary. Mei Sato and Peter A. Jumars (Darling Marine Ctr., Univ. of Maine, 193 Clark's Cove Rd., Walpole, ME 04573, mei_sato@umit.maine.edu)

Among migrating crustaceans, some species inhabiting shallow waters stay very close to the bottom or burrow into the sediments during the day and come up to the surface at night. This behavior, called emergence, was monitored by the Tracor Acoustic Profiling System (TAPS) with six frequencies $(265-3000 \mathrm{kHz})$ to clarify the mechanisms of benthic-pelagic coupling. Instead of one emergence event up to the surface at night, data with high spatial resolution indicate multiple emergence events varying with both the size of the organisms and distance above the bottom, suggesting that for some species there is a target depth beyond which upward migration is limited. In the Damariscotta River estuary, ME, nocturnal emergence events of mysid shrimp, Neomysis americana, occur from late June through early November. Mysid shrimp are known to be important food sources for fishes such as longhorn sculpins, smelt, alewives, and Atlantic cod. The timing of their emergence near the bottom corresponds to the maximum flood tide, suggesting enhancing retention in the estuary. Smaller organisms, on the other hand, emerge primarily during periods of slack tide, but reach the surface only at slack low, also avoiding washout.

\section{9:50}

2aAOa7. Combining acoustics and video to image fish in the acoustic dead zone. Jennifer L. Miksis-Olds, Ernest Bernard, Christopher J. Jakubiak, and D. V. Holliday (School for Marine Sci. and Technol., Univ. of Massachusetts, 838 S. Rodney French Blvd., New Bedford, MA 02744, jmiksis@umassd.edu)

A stationary acoustical-optical platform (AOP) has been developed to detect groundfish presence and to estimate biomass. The AOP is a new approach to fisheries science because sampling from a platform on the ocean floor overcomes limitations of traditional surface acoustic surveys, where echoes from groundfish near the seafloor are obscured by stronger reflections from the ocean bottom. The AOP capitalizes on the complementary strengths of acoustical and optical sampling techniques by using acoustics for fish enumeration and optics for species identification and measurements of fish length. Each AOP is equipped with four video cameras, two 200-kHz sonar transducers, and a suite of environmental sensors. One sonar face is mounted in the horizontal direction to image the area directly above the seafloor, whereas the second transducer is oriented vertically to sample the water column above the platform. Acoustic backscatter, target species, size, and observation angle were quantified when fish were simultaneously detected by the sonar and optical sensors. Calculations of target strengths were then related to fish size and orientation, for incorporation into biomass estimates. The AOP is designed to be used in an observation system with multiple platforms elements, so fish populations can be monitored over large spatial scales. [Work supported by NOAA.]

\section{0:05-10:20 Break}

\section{$10: 20$}

2aAOa8. Acoustic and optic characterization of forage fish distribution in the Bering Sea. Sandra Parker Stetter, John Horne (School of Aquatic and Fishery Sci., Univ. of Washington, Seattle, WA 98195-5020), and James Churnside (Natl. Oceanograph. and Atmospheric Administration Environ. Technol. Lab., Boulder, CO 80305)

Mesopelagic forage fish species are important components of the Bering Sea ecosystem, but information on species distribution and identity is limited. Using acoustics and optics [LIght Detection And Ranging (LIDAR)], this study compared distribution patterns detected by the two gears. Forage species distribution and quantity differed between shelf (6$100 \mathrm{~m}$ ) and slope $(6-100 \mathrm{~m}, 100-300 \mathrm{~m}, 300 \mathrm{~m}$ to bottom) regions. Acoustic results suggested that shallow and deep depth zones contained dispersed backscatter while the middle slope layer contained patchy schools associated with the shelf break. Variogram results for repeated LIDAR surveys of the shelf and slope regions indicated that backscatter distribution between 6 and $30 \mathrm{~m}$ was dynamic at the scale of days. This result was expected given the strong frontal nature of the area. When LIDAR results were compared with coincident acoustic transects on the shelf and slope, differences were found in gear detection of backscatter. Based on acoustic results, 25\%-63\% of forage fish in the shelf and slope regions were deeper than the LIDAR detection range. Although both LIDAR and acoustics are constrained to portions of the water column, the utility of remote sampling technologies is dependent on survey objectives. [Funding was provided by the North Pacific Research Board.] 
mechanics and the velocity field of fish groups previously estimated from OAWRS imagery using acoustic flow [Jagannathan et al., J. Acoust. Soc. Am. 119, 3435 (2006)]. These environmental stress fields describe the driving mechanism behind the dynamic behavior of large fish groups such as expansion, contraction, and translation. Regions of high stress are correlated with regions of high areal fish density. They are also correlated with density waves that convey information across a fish group. Our estimates of environmental stress on fish groups can be correlated with environmetal parameters such as plankton, temperature, and current distributions through physical and biological driving mechanisms.

\section{1:05}

2aAOa11. Sonar mapping for coral reef conservation. Lisa Zurk, Joe Lotz, Tim Ellis, James McNames (Elec. and Comput. Eng. Dept., Portland State Univ., P.O. Box 751-ECE, Portland, OR 97207, zurkl@cecs.pdx.edu), and Jean-Louis Ecochard (The Nature Conservancy, Arlington, VA 22203)

Many conservation efforts require an inexpensive and easy-to-use method of mapping and monitoring coral reef structure. A desirable product is a $3 \mathrm{D}$ map that effectively portrays the reef relief (and potentially the sub-structure) or features such as fish locations and fish spawning areas. Given field site limitations associated with the reefs, the mapping technique should be portable, rugged, and easy to use. One solution, developed at the Nature Conservancy (TNC), is to utilize a Lowrance fish finding sonar with a GPS system to estimate the top of the coral. This approach has been employed for TNC mapping exercises to generate ArcGIS maps of conservation areas. However, these maps are often negatively affected by sampling and processing artifacts such as sparse collection geometries (causing interpolation errors), distortion due to beam shape, and changes in boat (transducer) position due to wave motion. In this presentation we discuss recent work to address these effects, as well as enhancements to the system by processing acoustic returns from the coral substructure or within the water column for detection of fish populations. The presentation will provide a description of future research, including the intent of making the finished system available as a conservation tool. continental-shelf scale OAWRS images of areal fish population density [Science 311, 660-663 (2006)]. The calculation is based on continuum

\section{Invited Papers}

2aAOa12. Robust analysis of single echo trace obtained by split beam sounder. Masahiko Furusawa (Tokyo Univ. of Marine Sci. and Technol., 4-5-7 Konan, Minato Tokyo 108-8477 Japan, frsw@ s.kaiyodai.ac.jp) and Kazuo Amakasu (Natl. Res. Inst. of Fisheries Eng., Kamisu Ibaraki 314-0408 Japan)

The theory and method of the echo trace analysis of single echoes (ETA), which simultaneously analyze the shape and level of single echoes, are almost established. But, the method has not been extensively used. The major reasons are high-quality echoes with a high signal-to-noise ratio (SNR) necessary for the analysis are some times difficult to obtain; the error introduced by transducer motion is large; and conditions for the theory sometimes could not be fulfilled. Therefore, we apply the ETA to the split beam method, which is most advantageous for the ETA, and make the method robust by introducing the following: careful echo selection considering SNR, correction of the effect of transducer motion, smoothing of the trace by regression analyses, introduction of indexes checking reliability, etc. The useful results of our ETA are the track, the swimming speed and orientation, the target strength as a function of tilt angle, and the estimated size of individual fish. The robust ETA was applied to echoes of fish near a sea bottom and of Antarctic krill to confirm the effectiveness of the method.

\section{1:40}

2aAOa13. Comparing acoustic model estimations and in situ target strength measurements of juvenile walleye pollock (Theragra chalcogramma). Kazuhiro Sadayasu (Natl. Res. Inst. of Fisheries Eng., Fisheries Res. Agency, 7620-7 Hasaki, Kamisu, Ibaraki 314-0408, Japan), Tohru Mukai, and Kohji Iida (Hokkaido Univ., Hakodate, Hokkaido 041-8611, Japan)

Recently, many acoustic surveys for the juvenile walleye pollock have been done around the Hokkaido, Japan. Dependence of target strength on fish length is a most important factor in the conversion of acoustic data to fish biomass. However, the relation between target strength and length of juvenile walleye pollock is not well investigated. This study examined the target strength length relationship $(3-10 \mathrm{~cm})$ for the echo integration measurements of the juvenile walleye pollock at $38 \mathrm{kHz}$ from acoustic theoretical model predictions and in situ measurements. A prolate spheroid model was used to compute the target strength of the fish relative to its orientation. Swimbladder morphologies of live juvenile walleye pollock caught by set net were obtained from transmission of light or soft x-ray, and converted their morphological parameters. Biological samplings using framed mid-water trawl were carried out near 
the spawning area of walleye pollock. During fish samplings, in situ target strength measurements were conducted using quantitative echo sounder with split beam transducer. While predicted mean target strengths matched mean in situ measurements at small length, there was not agreement at the large length. This discrepancy is a result of avoidance of large fishes from a net mouth.

\title{
Session 2aAOb
}

\section{Acoustical Oceanography and Signal Processing in Acoustics: Acoustic Tomography for Coastal and Deep Water Applications III}

\author{
James A. Mercer, Cochair \\ Univ. of Washington, Applied Physics Lab., 1013 NE 40th St., Seattle, WA 98105-6698 \\ Arata Kaneko, Cochair \\ Hiroshima Univ., Graduate School of Engineering, 1-4-1 Kagamiyama, Higashi-Hiroshima, Hiroshima 739-8527, Japan
}

Chair's Introduction-7:45

\section{Contributed Papers}

\section{$7: 50$}

2aAOb1. Moving ship thermometry. James A. Mercer, Nick Alger, Bruce M. Howe, Rex K. Andrew (Appl. Phys. Lab., Univ. of Washington, 1013 NE 40th St., Seattle, WA 98105, mercer@apl.washington.edu), and John A. Colosi (Naval Postgraduate School, Monterey, CA 93943)

The long-range ocean acoustic propagation experiment (LOAPEX), conducted in the NE Pacific Ocean, provided acoustic transmissions from a ship-suspended source at seven stations spanning a near zonal path of $3200 \mathrm{~km}$. The transmissions were received on several bottom-mounted horizontal hydrophone arrays distributed about the NE Pacific Ocean Basin. The experiment and a simple inverse method are described and a preliminary thermal "snapshot" of the ocean basin is presented. [Work supported by ONR.]

\section{8:05}

2aAOb2. Anisotropy in the spatial distribution of the arrival points of a ray after a fixed travel time. Stanley M. Flatté (Phys. Dept., Univ. California Santa Cruz, Santa Cruz, CA 95064, sflatte@ucsc.edu) and John A. Colosi (Naval Postgraduate School, Monterey, CA 93943)

An acoustic pulse emitted from a point source arrives on a welldefined wavefront after a fixed travel time. In the geometrical-acoustics approximation, this behavior is understood by following rays. Two simple ray models are presented to treat the scattering of rays by internal waves, and to evaluate the ratio of movements in the ray end points within and perpendicular to the wavefront. Assuming weak forward scattering, and no waveguide, the model predicts that the end points move along the wavefront over much larger distances than perpendicular to the wavefront. [Work supported by ONR.]

\section{$8: 20$}

2aAOb3. Fresnel zones in inhomogeneous media. Irina I. Rypina and Michael G. Brown (Univ. of Miami, RSMAS-AMP, 4600 Rickenbacker Cswy., Miami, FL 33149)

A simple general expression for the width of a Fresnel zone in a smooth inhomogeneous environment is derived. In a stratified environment at long range, the general expression is shown to reduce approximately to one that is proportional to $\sqrt{|\alpha| r(R-r) / f}$, where $\alpha$ is the ray stability parameter, $f$ is the acoustic frequency, $r$ is the range to the field point of interest, and $R$ is the source-to-receiver range. In a stratified environment on which a weak perturbation is superimposed, at long range the width of a bundle of micromultipaths is shown to be approximately proportional to $|\alpha| \sqrt{r(R-r)}$. Thus, at long range both the diffractive and scattering contributions to "the width of a ray" are proportional to $\sqrt{r(R-r)}$ and increase with increasing $|\alpha|$. Theoretical predictions are shown to agree well with travel time sensitivity kernel calculations. [Work supported by ONR.]

\section{$8: 35$}

2aAOb4. Moving ship tomography from the Basin Acoustic Seamount Scattering Experiment (BASSEX-04). Kevin D. Heaney (OASIS Inc., 11006 Clara Barton Dr., Fairfax Station, VA 22039), Arthur B. Baggeroer (MIT, Cambridge, MA 02139), Peter F. Worcester, and Matthew A. Dzieciuch (Univ. of California at San Diego, La Jolla, CA 92093)

During the Basin Acoustic Seamount Scattering EXperiment (BASSEX-2004) a set of 32 acoustic transmissions of four moored sources in the central Pacific were made. Broadband transmissions $(100 \mathrm{~Hz}$ bandwidth, $250 \mathrm{~Hz}$ center frequency, $12.276 \mathrm{~s}$ period length) from two sources suspended (800 $\mathrm{m}$ depth) were received on a towed array $(350 \mathrm{~m}$ depth, $200 \mathrm{~m}$ aperture) as it passed between the two sources at a range of $250 \mathrm{~km}$. This data set provides the opportunity for examining the feasibility of underway moving ship tomography (MST). The primary oceanographic feature is the variability of the mixed layer over a $500 \times 500-\mathrm{km}^{2}$ region. Accurate ocean acoustic tomography requires the development of an $N$ $\times 2 \mathrm{D}$ inversion scheme as well as techniques to perform array element localization to within $10 \mathrm{~m}$. Results on applying a Kalman-filter-based technique to the array sensor data (depth, pitch, roll) and the measured ADCP will be presented. With the receiving ship transecting the path between the two source moorings, each transmission has two paths that pass through different bodies of water.

\section{$8: 50$}

2aAOb5. Vertical coherence of low-frequency sound waves propagating through a fluctuating ocean. Alexander G. Voronovich and Vladimir E. Ostashev (NOAA, Earth System Res. Lab., PSD4, 325 Broadway, Boulder, CO 80305)

Low-frequency sound waves propagating over large distances in the ocean exhibit significant scattering due to internal waves. Studies of lowfrequency sound propagation through a fluctuating ocean are important for many practical concerns such as communication, acoustic tomography, and source detection, ranging, and recognition. In a recent work [A. G. Voronovich and V. E. Ostashev, J. Acoust. Soc. Am. 63, 1406-1419 
(2006)] a 3-D modal theory of sound propagation through a fluctuating ocean was developed. Closed equations for the first two statistical moments of a sound field were derived using the Chernov method and assuming that the spectrum of internal waves is given by the Garret-Munk spectrum. In the present paper, we numerically solve the derived equation for the second moment. The results obtained are used to study the dependence of the vertical coherence of a sound field on several parameters of the problem: range, frequency, source and receiver depths, and parameters that characterize the Garret-Munk spectrum. [Work supported by ONR.]

\section{9:05}

2aAOb6. A partial theory of phase coherence loss in deep ocean propagation. Francisco J. Beron-Vera and Michael G. Brown (RSMAS-AMP, Univ. of Miami, 4600 Rickenbacker Cswy., Miami, FL 33149)

In a fluctuating ocean, coherently adding multiple receptions of repeated transmissions of a signal generally results in a processing gain relative to the background noise only for some finite duration owing to a gradual loss of wave-field phase coherence. In this talk a theoretical framework for understanding some elements of phase coherence loss in transient wave fields in deep ocean environments is presented. The theory predicts that phase coherence loss is pathdependent and very different at short range than at long range. PE simulations of transient wave-field phase coherence loss in an idealized time-varying ocean are presented and shown to be in good qualitative agreement with theoretical predictions. [Work supported by ONR.]

\section{9:20}

2aAOb7. Vertical structure and arrival times of the axial wave based upon conductivity, temperature, and depth data acquired during the Long-range Ocean Acoustic Propagation Experiment. Natalie S. Grigorieva and Gregory M. Fridman (Dept. of Appl. Math., St. Petersburg State Marine Tech. Univ., 3 Lotsmanskaya Str., St. Petersburg, 190008, Russia,nsgrig@natalie.spb.su)

In the LOAPEX experiment at some distances from VLA the sound source was deployed to depths quite close to the sound-channel axis. This took place at stations T1000, T500, T250, and T50 where, in particular, the source was deployed to $800 \mathrm{~m}$ and the depth of the sound-channel axis at these ranges is about $680 \mathrm{~m}$. The interference of the wave fields corresponding to near-axial rays leads to formation of a complex interference wave ("the axial wave") that propagates along the axis being a component of the axial crescendo. The talk will present the results on simulation of the axial wave as a function of depth of the receiver and the calculation of its arrival time at frequencies $56.25-93.75 \mathrm{~Hz}$. In the time domain the pulse propagation modeling is carried out for the broadband acoustic signals with the pulse center frequency of $75-$ and $37.5-\mathrm{Hz}$ bandwidth. The obtained results are compared with those for the lowest mode in the adiabatic approximation. Calculations are made for the range-dependent ocean model based upon LOAPEX CTD data and use the integral representation of the axial wave in a local coordinate system introduced in the vicinity of the range-variable sound-channel axis. [Work supported by ONR.]

\section{9:35}

2aAOb8. Evolution of a downslope propagating acoustic pulse. Kevin D. Heaney (OASIS, Inc., 11006 Clara Barton Dr., Fairfax Station, VA 22039), Arthur B. Baggeroer (MIT, Cambridge, MA 02139), Peter F. Worcester, Matthew A. Dzieciuch (Univ. of California at San Diego, La Jolla, CA 92093), James A. Mercer, Bruce M. Howe, and Rex K. Andrew (Univ. of Washington, Seattle, WA 98105)

During the North Pacific Acoustics Laboratory (NPAL-04) experiment broadband transmissions ( $50 \mathrm{~Hz}$ bandwidth, $75 \mathrm{~Hz}$ center frequency, 27.28 s period length) from a bottom-mounted source off the island of Kauai were received on a towed array ( $350 \mathrm{~m}$ depth, $200 \mathrm{~m}$ aperture) near the island and a large aperture vertical line array (VLA) at a range of 2469.5 km. Previous work [Heaney, J. Acoust. Soc. Am. 117(3), 1635-1642 (2005)] has demonstrated that bottom interaction near the source is a fun- damental process in the evolution of the wave-train. In this paper results from eight receptions at ranges from 2 to $300 \mathrm{~km}$ and the reception on the distant VLA is used to examine the wavefront evolution as it propagates into deep water. Results from a local geo-acoustic inversion will be presented as well as comparison of data with broadband parabolic equation simulations. The initial conclusion is that the first-order bottom bounce, off the seamount near the source, acts as an image source, contributing to a complex arrival pattern even at great distances.

\section{$9: 50$}

2aAOb9. Evolution of second-order statistics of low-order acoustic modes. Tarun K. Chandrayadula, Kathleen E. Wage (George Mason Univ., 4400 Univ. Dr., MSN 1G5, Fairfax, VA 22030, tchandra@gmu.edu), James A. Mercer, Bruce M. Howe, Rex K. Andrew (Univ. of Washington, Seattle, WA 98195), Peter F. Worcester, and Matthew A. Dzieciuch (UCSD, La Jolla, CA 92093)

Low-mode signals measured during long range tomography experiments, such as the Acoustic Thermometry of Ocean Climate (ATOC) and the North Pacific Acoustic Laboratory experiments, have a random arrival structure due to internal waves. At megameter ranges, the narrow-band mode amplitude is predicted to be Gaussian and uncorrelated with other modes [Dozier and Tappert, J. Acoust. Soc. Am. 63, 353-365 (1978)]. Wage et al. measured the centroid, frequency coherence, time spread, and time coherence for the broadband ATOC mode signals received at ranges exceeding $3000 \mathrm{~km}$ [Wage et al., J. Acoust. Soc. Am. 117, 1565-1581 (2005)]. The 2004 Long Range Acoustic Propagation EXperiment (LOAPEX) provided an opportunity to observe how the mode statistics evolves with range. This talk investigates the mean, temporal covariance, and intermodal correlation of the low modes at ranges between 50 and $3200 \mathrm{~km}$ using LOAPEX data. Broadband parabolic equation simulations were performed to model internal wave effects on the low-mode signals at the various LOAPEX stations. A kurtosis measure is used to study the amount of cross-modal scattering with respect to range. Statistics of the measured and simulated mode signals are compared. [Work supported by ONR.]

\section{0:05-10:20 Break}

\section{0:20}

2aAOb10. Modal group time spreads in weakly range-dependent deep ocean. Ilya A Udovydchenkov and Michael G Brown (RSMAS, AMP, Univ. of Miami, 4600 Rickenbacker Cswy., Miami, FL 33149)

Modal group time spreads in deep ocean environments are investigated. The environments considered consist of a range-independent background on which a range-dependent perturbation, due for example to internal waves, is superimposed. Modal group time spreads are defined to be the temporal spread of acoustic energy with a fixed mode number across a nonvanishing frequency band. It is shown that there are three contributions to modal group time spreads that combine approximately in quadrature. These contributions are (1) the recriprocal bandwidth, (2) a deterministic dispersive contribution, and (3) a scattering-induced contribution. Contributions 2 and 3 are shown to be controlled by the waveguide invariant $\beta(m, f)$, a property of the background sound speed structure. Numerical simulations are shown to be in good agreement with theoretical predictions. [Work supported by ONR.]

\section{0:35}

2aAOb11. Long-range ocean acoustic propagation experiment (LOAPEX) acoustic modeling analysis using measured environmental data. Michael Wolfson and Frank Henyey (Appl. Phys. Lab., Univ. of Washington, 1013 NE 40th St., Seattle, WA 98105-6698)

One objective of LOAPEX concerns the range dependence of multiple forward scattering for broadband, long-range acoustic transmissions. Using environmental data extracted during the experiment, a sound speed field is constructed, which includes sound speed fluctuations due to neu- 
trally buoyant intrusions as well as internal waves. This sound speed field is used as input to a full wave simulation model. Range-dependent results from simulations regarding the coherent field and depth scattering below lower turning point caustics are presented.

\section{0:50}

2aAOb12. A comparison of experimental and theoretical forward scattering by the Kermit-Roosevelt Seamount. Arthur Baggeroer, Henrik Schmidt, Joseph SikoraIII, Hyun Joe Kim (MIT, 77 Massachusetts Ave., Cambridge, MA 02139), Edward Scheer (Woods Hole Oceanograph. Inst., Woods Hole, MA 02543), Peter F. Worcester, Matthew A. Dzieciuch (Univ. of California at San Diego, La Jolla, CA 92093), and Kevin D. Heaney (OASIS Inc., Fairfax Station, VA 22039)

The SPICEX-LOAPEX-BASSEX experiments measured forward scattering from the Kermit-Roosevelt seamounts. LFM and M-sequence signals from $250-\mathrm{Hz}$ moored sources and a $75-\mathrm{Hz}$ ship-deployed source were received with Penn State University's 162-element FORA array. Receptions were obtained from these sources at ranges of hundreds to $1600 \mathrm{~km}$. Previous work [J. Acoust. Soc. Am. 117, (2549)] discussed the BASSEX forward shadow and patterns of horizontal refraction around the seamount. Additional work [J. Acoust. Soc. Am. 118, (1936)] compared experimental results with theory using a conical section model of a seamount. This presentation concerns the pulse compressed and array processed time series of $M$-sequence signals in the forward scattered field of the seamounts. Both travel times and angles of arrival are reconciled with 2-D ray-trace models. Results are comparable to refracted rays, which mainly propagate over the seamount. These, however, do not explain additional echoes, which appear in the experiment, possibly due to reflection and/or diffraction. Normal mode and PE methods are used to confirm ray models and identify additional echoes.

\section{1:05}

2aAOb13. On the forward problem of mode coupling through shallow-water nonlinear internal waves. John Colosi and Aaron Young (Dept. of Oceanogr., Naval Posgraduate School, Monterey, CA 93943)

Using the Dyson series, we have developed a 2-D (depth and range) theory to predict the coupling of low-frequency acoustic modes propagating through shallow water nonlinear internal wave fields. Parabolic equation numerical simulations with typical shallow water internal wave fields show that the theory can accurately predict mode coupling up to an acoustic frequency of about $300 \mathrm{~Hz}$. Second-order theory may extend the results to higher frequency and larger amplitude internal waves. Application of the theory to $3-\mathrm{D}$ problems in which azimuthal coupling is weak will be discussed.

\section{1:20}

2aAOb14. High-frequency current tomography in coastal regions. Mohsen Badiey and Entin Karjadi (College of Marine and Earth Studies, Ocean Acoust. Lab., Univ. of Delaware, Newark, DE 19716)

In deep-water tomography refracted ray paths can provide accurate account of travel time for the inverse problem. However, in shallow coastal regions, due to the geometry of the source-receiver as well as the waveguide dimensions, most acoustic energy is a multiple reflection from sea surface and sea bottom. This makes the forward problem which deals with resolving the multipath structure of the arriving signals difficult. In this paper, we discuss the limits of reciprocal acoustic transmission as a function of waveguide temporal and spatial parameters for the purpose of tomography application in coastal regions. Results from a high-frequency $(0.5-24 \mathrm{kHz})$ quasi-reciprocal tomography experiment in a very shallowwater coastal region are presented. Using these data, the accuracy and limit of current tomography for variable sea states are examined for different center frequencies and bandwidths. [Work supported by ONR.]

\section{$11: 35$}

2aAOb15. A multistation coastal acoustic tomography system with coherent operation. Hong Zheng (S.E.A. Corp., IU Bldg. 4F, 2-23 Shiohama, Ichikawa, Chiba 272-0127, Japan), Arata Kaneko, and Noriaki Gohda (Hiroshima Univ., Higashi-Hiroshima, Hiroshima 739-8527, Japan)

A multistation coastal ocean acoustic tomography (CAT) system with coherent operation has been developed and applied to field experiments over ten times in the last 8 years. The system is designed to measure both temperature and current velocity fields in the coastal ocean. As is well known, an ocean acoustic tomography is generally based on travel time measurements. In the CAT system, the clock accuracy for travel time measurement is most important to realize coherent transmission from multistations and to measure especially current velocity. For highly accurate coherent transmission from multistations, each station is equipped with a timing module synchronized by the clock signals of global positioning system (GPS). As a result, time error is maintained less than $0.1 \mu \mathrm{s}$. In the sound transmission from multistations, the code division multiple access (CDMA) and spread spectrum technology are applied. The coded signals also improved remarkably the signal-to-noise ratio of the CAT system. With detecting not only the amplitude, but also the phase of received signals, the accuracy of travel time measurement is improved within a sampling interval of data. This multistation CAT system served to map 2-D time-varying tidal current structures in Tokyo Bay (Kaneko et al., 2005).

\section{1:50}

2aAOb16. Nonlinear optimization for beamforming a geometrically deficient vertical line array: Application to sediment tomography. Steven E. Crocker (Naval Undersea Warfare Ctr., Newport, RI 02841), James H. Miller, Gopu R. Potty (Univ. of Rhode Island, Narragansett, RI 02882), and James F. Lynch (Woods Hole Oceanogr. Inst., Woods Hole, MA 02543)

The 1996 Shelfbreak PRIMER experiment included broadband acoustic transmissions in the vicinity of a shelfbreak front collected using a geometrically deficient vertical line array (VLA). The broadband signals were used to tomographically image sediment sound speed and attenuation. The VLA spanned the lower half of the water column. Hence, the orthonormal properties of the underlying normal modes cannot be guaranteed for any replica vector based on the local modal structure. This produces modal sidelobes in the output of the geometrically deficient VLA. Various methods of extracting the normal modes from VLA data have been developed including a generalized least squares method shown to improve the performance of a geometrically deficient VLA processing phase encoded tomography signals [Chiu et al., IEEE J. Ocean. Eng. 22, 522-533 (2005)]. The present study seeks to enhance the modal resolution for the reception of high-amplitude, impulsive signals transmitted over a wide variety of source receiver paths in shallow water where ensemble averaging is not an option. The method uses nonlinear optimization techniques to compute a set of array element coefficients that enhance suppression of modal sidelobes, while maintaining adequate spatial matching with the desired mode. [Work supported by Office of Naval Research.] 


\title{
Session 2aBB
}

\section{Biomedical Ultrasound/Bioresponse to Vibration: Topical Meeting on Shock Wave Therapy I}

\author{
Robin O. Cleveland, Cochair \\ Boston Univ., Aerospace and Mechanical Engineering, 110 Cummington St., Boston, MA 02215 \\ S. H. R. Hosseni, Cochair \\ Tohou Univ., Inst. of Fluid Science, 2-1-1 Katahira, Aoba, Sendai 980-8577, Japan
}

Invited Papers

\begin{abstract}
8:00
2aBB1. Extracorporeal cardiac shock wave therapy ameliorates myocardial ischemia in animals and humans. Hiroaki Shimokawa (Grad. School of Medicine, Tohoku Univ., Sendai 980-8574, Japan)

The number of patients with end-stage coronary artery disease without indication of coronary intervention or coronary artery bypass surgery has been increasing. Gene and/or cell angiogenic therapies are currently under investigation for those patients, however, the therapies are invasive in nature and their effectiveness and safety have not been established yet. We here report our successful development of noninvasive extracorporeal cardiac shock wave therapy (CSWT) for the treatment of myocardial ischemia. We first confirmed that endothelial expression of VEGF and its receptor Flt-1 peaks with a low level of SW at $0.09 \mathrm{~mJ} / \mathrm{mm}^{2}$ in vitro. We then demonstrated that CSWT effectively ameliorates myocardial ischemia in a porcine model of effort angina without any adverse effects. Finally, we were able to demonstrate that CSWT effectively ameliorates myocardial ischemia and related symptoms in patients with end-stage coronary artery disease without any adverse effects. Myocardial perfusion was improved with CSWT only in the ischemic region where we treated with the therapy. We are currently performing a double-blind clinical study to further confirm the usefulness and safety of our CSWT. [Work supported in part by grants from the Japanese Ministry of Health, Labor and Welfare.]
\end{abstract}

2aBB2. Various old and new shock-wave applications require different shock-wave beam characteristics. Reiner Schultheiss (Tissue Regeneration Technologies, LLC, 110 Arnold Mill Park, Ste. 400, Woodstock, GA 30188)

Four different medical applications of shock waves and the appropriate pressure field will be discussed. Lithotripsy requires maximum shock-wave energy at the stone. High-pressure amplitudes and the total acoustical energy are the preferable parameters discussed for the disintegration effect. Improved kidney stone disintegration coincident with reduced side effect on the nephritic tissue was realized by a low-angle focusing ellipsoid (LithoGold). In the treatment of soft tissue, unfocused shock waves allow the mechanical parameters like peak pressure and the energy density to be lowered dramatically. Activation of the body's healing mechanisms are found after low pressure/ energy application by the release of growth factors, increased number of blood vessels, and the formation of new true tissue instead of scar tissue. For the treatment of diabetic skin ulcers an unfocused shock head is in controlled clinical studies within the USA. The last application relates to treating open heart muscle and requires a very small shock-wave head. A very short focal distance $(11 \mathrm{~mm})$ is used. New blood vessels and the formation of new heart muscle cells are found after treatment. Technical and medical reasons for the different shock-wave beam characteristics are provided.

\section{8:40}

2aBB3. Shock waves in orthopedics. Takashi Saisu (Div. of Orthopaedic Surgery, Chiba Children's Hospital, 579-1 Heta-chou, Midori-ku, Chiba 266-0007, Japan, h-saisu@dc4.so-net.ne.jp)

Extracorporeal shock wave therapy (ESWT) in the orthopedic field was first reported in 1991 by Valchanou, for the treatment of pseudoarthrosis after fracture. Since 1995, many authors have reported on ESWT for the treatment of painful disease such as calcifying tendinitis of the shoulder, tennis elbow, or planter fasciitis. Now, it has become one of the major treatment methods in orthopedics and veterinary medicine, especially for racehorses. The shock wave research group at Chiba University has been studying ESWT since 1993. We have been elucidating mechanisms of action to the bone and the neural system, performing basic studies using rabbits and rats. A shock wave has three different effects, which are bone induction after multifocal microfracture, perforation of calcified tissue, and induction of degeneration of free nerve ending. Each effect has been utilized for the treatment of pseudoarthrosis, calcifying tendinitis of the shoulder, and enthesopathy such as tennis elbow or planter fasciitis, respectively. Considering our experiences in animal studies, future applications of ESWT would be noninvasive thickening and lengthening of bone and nonsurgical acetabular plasty to prevent osteoarthritis of the hip. Recently, we have been trying nonviral gene transfer assisted by shock waves and we have succeeded in a preliminary animal study. 
2aBB4. Influence of focusing and signal shape on the efficiency of stone fragmentation in vitro under equal energy conditions. Rainer E. Riedlinger, Marko Liebler, and Thomas Dreyer (Univ. Karlsruhe, IHE-Akustik, Kaiserstr. 12, D-76131 Karlsruhe, Germany, rainer.riedlinger@ihe.uka.de)

To investigate the mechanisms of fragmentation in lithotripsy, simulations and experiments were performed. Wave shapes deduced from measurements near the focus of a piezoelectric source were used as input signals for finite element calculations of stresses and strains in spherical stones of different sizes. The effects of strongly and weakly focused sound fields were compared to those of a plane wave assumption representing lithotripters with large focal widths. The energies of the fields were matched. In targets with diameters less than $6 \mathrm{~mm}$ the simulated tensile stresses induced by strong and weak focusing exceed those of the plane wave assumption. For in vitro experiments a double-layered self-focusing piezoelectric source was used. To represent lithotripters with different sound fields the signal shape was varied by changing the interpulse delay but maintaining the energy. The total number of pulses needed for fragmentation into particles smaller than $2 \mathrm{~mm}$ diameter was counted. At usually applied energies experimental results show that the numbers of pulses needed for complete fragmentation are almost independent of the signal shape. In conclusion, the results indicate that strongly focused lithotripters are in principle more effective than those with wide focal widths in breakage of small stones.

9:20

2aBB5. Using shock waves and micro-nanostructures for gene and drug delivery. Mark Kendall (Australian Inst. for Bioengineering and Nanotechnology (AIBN), Univ. of Queensland, Level 6, Queensland Bioscience Precinct (Bldg. 80), Brisbane, Brisbane Qd 4072 Australia)

Efficiently and safely delivering biomolecules to the skin's immunologically sensitive cells holds the promise of advancing radical new drugs, vaccines, and immunotherapies for major diseases [D. Chen et al., Expert Rev Vaccines 1(3): 89-100 (2002)]. Here I present research into using shock waves and micro-nanostructures to meet this goal. First, I analyze the physiology and immunology of the skin target and then introduce current physical approaches for targeting these cells for the immunotherapy [M. A. F. Kendall, Vaccine May 22; 24(21): 4651-656 (2006)]. Then, I focus on biolistics-accelerating with a helium flow micro-particles coated in DNA to ballistically penetrate the human skin in order to achieve an immunological effect [M.A.F. Kendall, Shock Waves J., 12(1), 22-30 (2002)]. I present key findings on the engineering of these devices, skin physical interactions induced by micro-particle impact and the resultant local biological responses. Finally, I introduce a micro-nanoprojection array patch technology, highlighting key advantages over biolistics, including the prevention of cell-death from shock impact with key cells.

\section{Contributed Papers}

9:40

2aBB6. Measurement of reduced stress in model kidney stones with increased rate of shock wave delivery in lithotripsy. Teiichiro Ikeda, Michael R. Bailey, Brian MacConaghy, Lawrence A. Crum (Ctr. for Industrial and Med. Ultrasound, Appl. Phys. Lab., Univ. of Washington, 1013 NE 40th St., Seattle, WA 98105), and Yoichiro Matsumoto (The Univ. of Tokyo, Bunkyo-ku, Tokyo 113-8656, Japan)

Slow clinical shock wave rates more effectively comminute stones. Higher rates create more cavitation bubbles along the focusing axis. Bubble clouds potentially reflect or attenuate the shock wave and also may collapse less energetically. Here, high-speed photo-elastography was used to visualize the dynamic stress distribution inside a transparent model stone. Photo-elastography records constant-stress lines, making quantification possible. PVDF sensors $(4 \mathrm{~mm}$ diameter) measured force on the proximal face of the stones. The impulsive force of the shock wave and the cloud collapse at various clinical rates (single shocks, $1 \mathrm{~Hz}, 2 \mathrm{~Hz}, 3$ $\mathrm{Hz}$ ) in degassed and non-degassed water were calculated from the measurements. Impulse forces from the shock wave and cavitation collapse were comparable in the range $4-7 \times 10^{-4} \mathrm{Ns}$. At clinical rates in gassaturated water, the stress fringes of the tensile component of the shock wave were reduced; the observable maximum stress was decreased; and impulsive force from the shock and the cavitation were decreased over single shocks. The results are evidence of reduced stress in the stone at higher rates due to attenuation by bubbles and less so to softened cavitation collapse. [Work supported by Grant Nos. NIH DK43881 and NSBRI SMS00402.]
9:55-10:10 Break

10:10

2aBB7. Numerical study of the collapse of a bubble subjected to a lithotripter pulse. Eric Johnsen and Tim Colonius (Div. of Eng. and Appl. Sci., California Inst. of Technol., 1200 E. California Blvd., Pasadena, CA 91125, ejohnsen@ caltech.edu)

In shock wave lithotripsy, the combined effect of focused shock waves and cavitation pulverizes kidney stones. Although cavitation has been shown to play an important role in the stone comminution process, the underlying mechanism has yet to be fully understood. The goal of the present study is to quantify the potential damage caused by the collapse of a single bubble near a solid surface. Using a high-order accurate quasiconservative, shock- and interface-capturing scheme [E. Johnsen and T. Colonius, J. Comput. Phys., in press (2006)], the response of an air bubble subjected to a lithotripter pulse is considered. In particular, quantities important in cavitation erosion, such as wall stresses and reentrant jet velocity, are measured as functions of the properties of the pulse (amplitude, wavelength) and the geometry of the problem (stand-off distance from the wall, presence of neighboring bubbles). Preliminary two-dimensional results for a "cylindrical bubble" show that a large water-hammer pressure is measured along the wall. This pressure increases for smaller stand-off distances and longer wavelengths. Further results for spherical bubbles will be presented at the meeting. [This work was supported by NIH Grant PO1 DK043881.] 
obtained. As a preliminary experiment, the device was applied to evaluate the enhancement of chemotherapeutic effects of shock wave in vitro, using

2aBB8. The effects of plane shock wave on the endothelial cells in vitro and the numerical analysis of pressure wave in the cell. Masaaki Tamagawa, Seiya Iwakura, Shiro Suetsugu, and Jun Fujii (Kyushu Inst. of Technol., Hibikino 2-4, Wakamatsu-ku, Kitakyushu, Fukuoka 808-0196, Japan, tama@life.kyutech.ac.jp)

Shock wave phenomena in living tissues are being applied in the fields of tissue engineering. It is expected that the elastic cells such as endothelial cells and cardio cells have a potential to regenerate or grow quickly by using high pressure wave and short duration time from $1 \mu \mathrm{s}$ to $100 \mathrm{~ms}$. There are two mechanisms for this, one is the effect of shock waves and the other is the effect of cavitation bubbles. In this paper, to investigate only the effects of plane shock waves on the endothelial cells in vitro, the cells worked by shock waves are observed by microscope, and FEM numerical simulation is done to analyze the mechanism. For experiments, the shock tube apparatus is used that makes plane waves and has several tens of ms duration time. After working shock waves (peak pressure 0.4 MPa) on suspended cells, $10 \%$ of them are disintegrated, but the others increase faster than control cells in vitro. The growth of cells by plane shock waves is also confirmed by the fact that activated gene expression is enhanced. Numerical simulation results show the gradient of the shear stress wave is an important factor.

\section{0:40}

2aBB9. Acoustic coupling can be problematic in shock wave lithotripsy. Yuri A. Pishchalnikov, James A. McAteer, Joshua S. Neucks, R. Jason VonDerHaar, Irina V. Pishchalnikova, and James C. Williams, Jr. (Dept. of Anatomy and Cell Biol., School of Medicine, Indiana Univ., 635 Barnhill Dr., Indianapolis, IN 46202-5120, yura@ anatomy.iupui.edu)

Research to improve lithotripsy has yielded considerable progress in determining mechanisms of SW-action, has led to development of innovative SW-delivery systems, and has offered practical strategies to enhance stone breakage and minimize renal injury. Little attention has been paid to how SWs are coupled to the body. In vitro tests were performed to assess the quality of coupling with a clinical Dornier DoLi-50 lithotripter. A test tank with a Mylar window was coupled to the treatment head using lithotripsy gel. Application of gel as performed in patient treatment always trapped air pockets at the interface, and it took considerable manipulation to eliminate them. Stone breakage was less efficient when air pockets were present, with a linear correlation between percent area of air-pocket coverage and reduction in breakage $(p<0.001)$. Breaking contact and recoupling —as can also occur in patient treatment-reduced stone breakage to $\sim 10 \%$ of air-free coupling. Air pockets created when gel was applied to both surfaces, or with coupling and recoupling, reduced the energy density of the pulse to $\sim 30 \%-50 \%$ of air-free. Thus, even under controlled conditions, acoustic coupling in SWL can be inefficient. Poor acoustic coupling could be a significant source of variability in clinical shock wave lithotripsy.

\section{0:55}

2aBB10. Experimental application of Holmium:yttrium-aluminumgarnet laser-induced shock wave as a drug delivery system. Tomohiro Ohki, S. H. R. Hosseini, Kazuyoshi Takayama (Biomed. Eng. Res. Organization, Tohoku Univ., Sendai, Japan), Junichi Sato, Atsuhiro Nakagawa, Teiji Tominaga (Tohoku Univ. Grad. School of Medicine, Sendai, Japan), Mariko Kambe (Senseki Hospital, Ishinomaki, Japan), and Mingu Sun (Tohoku Univ., Sendai, Japan)

One of the possible applications of shock waves is enhancement of drug delivery in the central nervous system. To achieve such a goal, it is necessary to develop a suitable shock wave source with the ability to be integrated with an endoscope or a catheter. We have developed a shock wave generator using a pulsed Ho:YAG laser as the energy source; this device is remarkable for its ability to expose shock wave to target tissue in a localized area. The physical feature and characteristics of the generator were clarified. The overpressures of Ho:YAG laser-induced cavitational shock waves were measured by using a PVDF needle hydrophone. Maximum overpressure of $19 \mathrm{MPa}$ at 4-mm distance from tip of the device was human gastric cancer cell (GCIY cell) line and an anticancer drug (Bleomycin: BLM). Proliferation rate of the cells decreased to $90 \%$ and $45 \%$, after application of 4000 and 6000 shots of shock waves at the BLM concentration of $1 \mathrm{mic}-\mathrm{g} / \mathrm{ml}$. Inhibitions in proliferation rate were observed in accordance with the number of shock wave applications and BLM concentrations.

\section{1:10}

2aBB11. Experimental study of micro shock wave focusing for precise medical applications. S. H. R. Hosseini, Tomohiro Ohki, and Kazuyoshi Takayama (Biomed. Eng. Res. Organization, Tohoku Univ., 2-1-1 Katahira, Aoba, Sendai 980-8577, Japan)

For applying shock waves to sensitive medical procedures like cranioplasty in a close vicinity of the brain or treatment of myocardial dysfunction, generation of micro underwater shock waves plays an important role. Such delicate applications make limits on usage of conventional shock wave (SW) sources. In the present research a half-ellipsoidal cavity with 20.0-mm minor diameter and the ratio of major to minor diameters of 1.41 was designed as a compact extracorporeal SW source. Silver azide $\mathrm{AgN}_{3}$ pellets ranging from 1.0 to $20 \mu \mathrm{g}$ with their energy ranging from 1.9 to $38 \mathrm{~mJ}$ were used to generate shock waves at the first focal point $F 1$ inside the reflector. Irradiation of a Q-switched Nd:YAG laser beam through a $400-\mu \mathrm{m}$ optical fiber was used to ignite the pellet. The whole sequences of the shock wave generation, propagation, and focusing were visualized by quantitative double pulse holographic interferometry and time-resolved high speed shadowgraph methods. Pressure histories were measured at the shock wave focusing area $F 2$ by using a fiber optic probe hydrophone. It is concluded that the compact ESW source has suitable characteristics for medical applications.

\section{$11: 25$}

2aBB12. Photo-elastic, high-speed images of stress induced in cylindrical model kidney stones by lithotripsy. Brian MacConaghy, Teiichiro Ikeda, Michael R. Bailey, Adam D. Maxwell (Ctr. for Industrial and Medical Ultrasound, Appl. Phys. Lab., Univ. of Washington, 1013 NE 40th St., Seattle, WA 98105), and Oleg A. Sapozhnikov (Moscow State Univ., Moscow, 119992, Russia)

Recent experiments and calculations show that a focusing shear wave generated by the shock wave traveling along the length of a cylindrical stone creates the dominant stress and causes fracture of cylindrical model stones. A small disk placed on the proximal face of the stone suppressed the longitudinal wave responsible for spallation in calculations yet had little effect on the number of shock waves required to fracture the stone in experiment. However, a disk placed around the stone blocked the shock wave traveling along the stone in calculations and suppressed fracture in measurements. The conclusion was that so-called dynamic squeezing was a dominant mechanism to spallation. Here, photo-elastic, high-speed imaging was used to observe the suppression of various waves in cylindrical stones made of acrylic. Glycerol was used to avoid artifact from the curvature of the stone. Comparison was made to calculations using the elasticity equations for an isotropic medium. Agreement between measurement and calculation was excellent and supports dynamic squeezing. The results help validate the model, and the technique and modeling may help us understand where and how stress is created in other shock wave therapies. [Work supported by NIH DK43881 and NSBRI SMS00402.]

\section{1:40}

2aBB13. Shock-wave-based biolistic apparatus. Viren Menezes, S. H. R. Hosseini, Kazuyoshi Takayama (Biomed. Eng. Res. Organization, Tohoku Univ., Sendai, Japan), Mutsumi Nakada, and Akira Kanno (Tohoku Univ., Sendai, Japan)

A shock-wave-based biolistic device has been developed to deliver DNA/drug-coated micro-projectiles into soft living targets. The device consists of an $\mathrm{Nd}$ :yttrium aluminum garnet laser, equipped with a suitable 
optical setup and a thin aluminum (Al) foil (typically $100-\mu \mathrm{m}$ thick). The powdered vaccines to be delivered are deposited on the anterior surface of the foil and the posterior surface of the foil is ablated using the laser beam. The ablation launches a shock wave through the foil that imparts an impulse to the foil surface, due to which the deposited particles accelerate to high velocities that are sufficient to enable them to penetrate soft targets.
The device has been tested for in vivo DNA delivery by delivering plasmid-coated, 1- $\mu \mathrm{m}$ size, gold $(\mathrm{Au})$ particles into onion and tobacco cells. The GUS activity was detected in the onion and tobacco cells after the addition of an artificial substrate. The present device is totally nonintrusive in nature and has a potential to get miniaturized to suit the existing medical procedures for drug delivery.

\title{
Session 2aEA
}

\author{
Engineering Acoustics: Ultrasonic Functional Devices I \\ Stephen C. Thompson, Cochair
Pennsylvania State Univ., Applied Research Lab., P.O. Box 30, State College, PA 16804 \\ Minoru Kurosawa, Cochair \\ Tokyo Inst. of Technology, G2-32, 4259 Nagatsuta, Midori-ku, Yokohama 226-8502, Japan
}

Invited Papers

8:00

\begin{abstract}
2aEA1. Ultrasonic motors-breakthrough trials for the next generation. Yoshiro Tomikawa, Chiharu Kusakabe, Hideki Tamura, Seiji Hirose (Dept. of Elec. Eng., Yamagata Univ., Yonezawa, Yamagata 992-8510, Japan, tomikawa@yz.yamagata-u.ac.jp), Takehiro Takano (Tohoku Inst. of Tech., Sendai, Miyagi 982-8577, Japan), and Manabu Aoyagi (Muroran Inst. of Tech., Muroran, Hokkaido 050-8585, Japan)

Twenty years and more have passed since real research of ultrasonic motors was started and now their practical applications are performed in some engineering fields. Therefore, such a problem has happened now as to what kinds of breakthrough ultrasonic motors must be researched for the next generation. For this reason, we are challenging the breakthrough research of the ultrasonic motors. This paper deals with such breakthrough motors. In the first part, we deal with thin-form ultrasonic motors of high revolution speed for their use as HDD motors, applying lead-free piezoelectric single crystal of $\mathrm{LiNbO}_{3}$. The second part contains some gyro-moment motors, devised by us originally. The motors can be driven electromagnetically and, moreover, electrostatically in theory. Therefore the motors are suited to ones fabricated by MEMS technology and might be applied as a micro-fun motor for heat radiation in electronic apparatuses, a windmill motor in fuel cells, and so on.
\end{abstract}

8:20

2aEA2. A constant beamwidth transducer for ultrasonic applications. Kim C. Benjamin, A. Lee Van Buren, Jeffrey A. Szelag, and Sheridan Petrie (Naval Undersea Warfare Ctr., 1176 Howell St., Newport, RI 02841)

A new class of transducers that produce uniform wide beams $(\sim 20 \mathrm{deg})$ at ultrasonic frequencies $(\sim 1-2 \mathrm{MHz})$ is being developed by the US Navy for ultrasonic applications. These transducers will provide a wide insonified field of view for twodimensional ultrasonic imaging arrays. The design features a larger volume of active material, relative to conventional planar designs, which allows the application of higher drive fields. The prototype described in this article was driven to 200 VRMS and produced a sound pressure of $203(\mathrm{~dB}$ re Pa at $1 \mathrm{~m}$ ) at a frequency of $2.0 \mathrm{MHz}$. The details of the ultrasonic constant beamwidth transducer (CBT) design, its fabrication, and measured performance are presented. The ultrasonic CBT described in this paper will soon be incorporated into the Navy's Underwater Sound Reference Division (USRD)'s Transducer Standards Loan Program.

\section{Contributed Papers}

$8: 40$

2aEA3. Traveling wave power in an energy circulation surface acoustic wave motor stator. Minoru Kurosawa, Yoshito Miyazaki, and Takashi Shigematsu (Tokyo Inst. of Technol., G2-32, 4259 Nagatsuta, Midori-ku, Yokohama 226-8502, Japan)

Surface acoustic wave (SAW) motors have been demonstrated their high performance as micro linear motor in speed, thrust, fine stepping motion, and so on. For application of the SAW motor to some equipment, however, the power consumption will be a problem in spite of the superior performance due to the limited power resource of a system. To avoid the large power consumption, an energy circulation driving method has been proposed and tested. The circulation factor will be discussed as concerns the performance at the energy circulation electrode and the phase shift in the system. For example, in case of the $90 \%$ circulation coefficient at the circulation electrode, eight times power of the driving power circulates as a traveling wave. But the 45 deg phase shift causes a reduction of onefourth of the traveling wave. The energy circulation of the SAW motor will be discussed quantitatively. [Research was supported by the Ministry of Education, Culture, Sports, Science and Technology, Grant-in-Aid for Science Research.] 
nol sensor that functions at high temperature. The SH-SAW propagation characteristics were calculated using a numerical calculation method considering temperature coefficients of the substrate and liquid. Calculation results indicate that the SH-SAW sensor can detect methanol solutions at temperature greater than $50^{\circ} \mathrm{C}$. Moreover, the calculated results suggest that the sensor sensitivity increases with increasing temperature. The methanol solutions were measured using the SH-SAW sensor. Concentration and temperature of the methanol solution were varied. Linear relationships between sensor responses and concentration were observed at each temperature. As the numerical calculation results suggest, the sensor sensitivity increases with increased temperature. Based on the experimental results, the detection limit of the sensor was estimated as $0.10 \%$ by weight at $60^{\circ} \mathrm{C}$.

\section{9:25}

2aEA6. An ultrasonic linear motor using traveling waves of twin ridge. Osamu Koyama, Daisuke Koyama, Kentaro Nakamura, and Sadayuki Ueha (P and I Lab., Tokyo Inst. of Technol, 4259-R2-25 Nagatsutacho, Midori-ku, Yokohama-shi, Kanagawa 226-8503, Japan, shukoyama@sonic.pi.titech.ac.jp)

An ultrasonic linear motor using ridge-mode traveling waves on a substrate is proposed for precision positioning. In our experiments, two parallel ridges are machined on an alumina ceramic substrate with the spacing of $19 \mathrm{~mm}$. The width, height, and length of the ridge are $3 \mathrm{~mm}, 9$ $\mathrm{mm}$, and $186 \mathrm{~mm}$, respectively. Two piezoelectric elements, $19 \mathrm{~mm}$ in length and $5 \times 5 \mathrm{~mm}^{2}$ in cross section, were installed at the position of 15 $\mathrm{mm}$ from each end of the ridge, and excited at $87.136 \mathrm{kHz}$ in their fundamental longitudinal mode to stimulate the fundamental ridge mode. Elliptical particle motions are formed, due to traveling waves along the ridge waveguides by the phased drive of the two transducers. An alumina ceramic slider pressed to the two waveguides is moved at $13 \mathrm{~mm} / \mathrm{s}$ in the opposite direction to the traveling wave through the friction forces. The moving direction can be switched by changing the propagation direction of the traveling wave. The detailed characteristics are experimentally studied. $\mathrm{LiTaO} 3$ is a shear horizontal $(\mathrm{SH})$ mode, the liquid-phase SAW sensor is realized using a crystal. In this paper, we present a SH-SAW-based metha-

\section{Invited Papers}

2aEA7. An application of surface acoustic wave for micro fluid systems. Hiroki Kuwano, Masato Sato, Jooohyng Bae, and Sumito Nagasawa (Grad. School, Tohoku Univ., 6-6-01 Aza-Aoba, Aramaki Aoba-ku, Sendai, Japan)

This paper describes an application of surface acoustic wave for micro fluid systems. Owing to its high sensitivity, fabrication easiness, and actuation capacity, current SAW devices are widely used as a chemical sensor and linear motor [Takahashi et al. IEEE Trans. Ultrason. Ferroelectri. Frequ. Control 43, 901 (1996)] as well as SAW devices including filters and SAW-Tag in the field of mobile communication. Furthermore, its applications for actuators in liquid such as liquid streaming [Shiokawa et al., Jpn. J. Appl. Phys. 28(28-1), 126-128 (1989)] and atomizer [Kurosawa et al., Sensors Actuators A 50, 69 (1995)] are very attractive for micro fluid systems such as a micro injector, a micro total analysis system, and a lab-on-chip. In this paper, we propose and evaluate a novel fluid actuator device for micro fluid systems using Rayleigh waves. One of the important issues is a micro pump and fluidic friction of the micro channel for TAS. The surface acoustic wave is one solution for the issue. Some of the fluid dynamics will be discussed.

10:00-10:20 Break

10:20

2aEA8. Phased arrays and devices for high-intensity focused ultrasound. Greg T. Clement (Harvard Med. School, 221 Longwood Ave., Boston, MA 02115)

Focused ultrasound (FUS) is rapidly attracting attention as an alternative to surgery for the treatment of tumors and other disorders. Such therapeutic procedures were first proposed over 60 years ago, but have only recently become clinically feasible. These new successes can be attributed primarily to two advances: improvement in noninvasive monitoring techniques and the development of high-power ultrasound arrays. As the field progresses, there is a demand for increased transducer bandwidth, allowing tissuespecific frequency optimization, and even imaging, while maintaining high acoustic power outputs. These arrays have been made possible via dice-and-fill manufactured 1-3 piezocomposite arrays. Transducers with over 500 elements have been tested in the clinic, 
and designs have been proposed for at least 5000 elements. Meanwhile, the rapid development of high-power, broadband IC technology has made it possible to build electronic components for amplifiers and switching at a practical cost (under $\$ 50 /$ channel). This talk will present several devices being developed in the Harvard/BWH FUS lab, in conjunction with the newly formed National Center for Image Guided Therapy. The challenges and limitations of operating at high driving intensities will also be addressed, with particular attention to nonlinear restoring behaviors of arrays driven off-resonance. [Work supported by NIH Grant U41 RR019703.]

\section{Contributed Papers}

10:40

2aEA9. Development of miniature ultrasonic probe with piezoelectric poly-crystalline film deposited by hydrothermal method. Tomohito Hasegawa, Akito Endo, Norimichi Kawashima, Shinichi Takeuchi (Toin Univ. of Yokohama, 1614 Kurogane-cho, Aoba-ku, Yokohama, Kanagawa 225-8502, Japan), Mutsuo Ishikawa, and Minoru Kurosawa (Tokyo Inst. of Technol., 4259 Nagatutamati, Midori-ku, Yokohama, Kanagawa 226-8502, Japan)

Recently, a PZT poly-crystalline film deposited on titanium substrate using the hydrothermal method was studied actively in our laboratory for development of an ultrasonic sensor. These PZT poly-crystalline films can form PZT poly-crystalline films easily on substrates of complex shape using hydrothermal method. We reported a needle-type miniature hydrophone and the ultrasonic transducer with hydrothermally synthesized PZT poly-crystalline films. Results confirmed that the hydrothermally synthesized PZT poly-crystalline films showed wideband frequency characteristics in the megahertz band of sensitivities. A miniature ultrasonic probe with wide frequency band characteristics of sensitivities is thought to be producible using hydrothermally synthesized PZT poly-crystalline films. Miniature ultrasonic probes were fabricated using hydrothermally deposited PZT poly-crystalline film on the end of 0.6-mm-diam titanium wires. Ultrasonic imaging experiments of a target in water were performed using a fabricated ultrasonic probe with hydrothermally synthesized PZT films. Consequently, it was confirmed that this probe has a center frequency of $16 \mathrm{MHz}$, a bandwidth of $80 \%$, and distance resolution more than $80 \mu \mathrm{m}$. We report the structure and performance of fabricated ultrasound probes with hydrothermally synthesized PZT poly-crystalline films, along with results of imaging experiments performed in water.

\section{0:55}

2aEA10. Study of a micro-tactile sensor with longitudinal vibrator in a smart micro-ultrasonic scalpel. Shunsuke Uchikawa and Minoru K. Kurosawa (Dept. of Information Processing, Tokyo Inst. of Technol., Mailbox: G2-32, 4259 Nagatsuta, Midori-ku, Yokohama, Japan)

A smart micro-ultrasonic scalpel using a hydrothermal PZT film vibrator has been proposed and fabricated. The micro-scalpel has two functions: in addition to a micro-ultrasonic scalpel, a micro-tactile sensor to indentify tumors. The tactile sensor function is studied. The resonance frequency of the vibrator changed when the vibrator tip made contact with a rubber sample. Irrespective of compliance of the sample, the resonance frequency increased up till about tens of micrometer sample. Then, against further sample deformation by the tip, the resonance frequency decreased. From the theoretical analysis using mechanical contact modeling [K. L. Johnson, Contact Mechanics (Cambridge University Press, Cambridge, 1985], it was found that the resonance frequency drop was caused by increase of the damping effect of the sample due to deeper stick of the vibrator tip. The Young's modulus of samples is able to be estimated from the resonance frequency curve. From the experimental result, there was a difference in the resonance frequency between a soft rubber sample and a hard one. The elastic characteristic of the sample will be quantified.

\section{1:10}

2aEA11. The TDK model for micromachined capacitive Lamb wave transducers. Li-Feng Ge (School of Electron. Sci.e and Technol., Anhui Univ., Hefei 230039, China)

Electrostatic or capacitive ultrasonic transducers (EUTs or CUTs) were originally developed from the condenser transmitters and microphones with grooved and roughened backplates in the 1930s. Significant progress in theoretical and experimental research has been made since the introduction of micromachining techniques at the end of the 1980s. We have seen the advancement not only from the roughened ultrasonic transducers to the 2-D array micromachined capacitive ultrasonic transducers (mCUTs), but also from the grooved ultrasonic transducers to the micromachined capacitive Lamb wave transducers (mCLWTs). Thus, the theoretical TDK model originally developed for grooved ultrasonic transducers [L.-F. Ge, Proceedings 16th Intl. Conf. Acoustics/135th ASA Meeting, 1081-1082, 1998; and Sciences in China (A), (in English), 42, 1308-1315 (1999)] can be used for mCLWTs. It is most important for a reliable model to be able to predict the transducer's natural frequencies, at which the lowest-order antisymmetric Lamb wave ( $A_{0}$ wave) is excited. As an example using a typical transducer [M. H. Badi et al., IEEE Trans. Ultrason. Ferroelectr. Freq. Control 50(9), 1191-1203 (2003)], the fundamental frequency predicted using the TDK model is $2.18 \mathrm{MHz}$, which agrees well with the measured result of 2.1 MHz. [Work supported by NSFC (60374044).] 


\title{
Session 2aED
}

\section{Education in Acoustics: Demonstrations and Tools in Acoustics Education}

\author{
Uwe J. Hansen, Cochair \\ 64 Heritage Dr., Terre Haute, IN 47803 \\ Fumiaki Satoh, Cochair \\ Chiba Inst. of Technology, Dept. of Architecture and Civil Engineering, 2-17-1 Tsudanuma, Narashino, \\ Chiba 275-0016, Japan \\ Chair's Introduction-9:00 \\ After the Chair's Introduction, the authors of each paper will briefly describe their demonstrations ( 2 minutes each). Authors will \\ then be at their demonstrations to interact with interested participants for the remainder of the session.
}

\section{Invited Papers}

2aED1. Visualization of acoustic resonance phenomena using Kundt's dust figure method. Shinichi Sakamoto, Takumi Asakura, Kanako Ueno (Inst. of Industrial Sci. Univ. of Tokyo, Komaba 4-6-1, Meguro-ku, Tokyo, 153-8505, Japan, sakamo@iis.u-tokyo.ac.jp), Yu Sakimoto, Fumiaki Satoh, and Hideki Tachibana (Chiba Inst. of Technol., Narashino-shi, Chiba, 275-0016, Japan)

It is very effective to visualize a sound field for intuitive understanding of various acoustic phenomena, especially for acoustic education. The most famous and classical visualization technique is the Kundt's dust-tube method contrived by August Adolph Kundt. He devised this experimental technique to determine the sound velocity in the air by observing the mode pattern of a standing wave excited in a glass tube. The technique can be applied to various other acoustic resonance phenomena. In Japan, Sato and Koyasu applied this technique to a two-dimensional room acoustic model experiment in which the effect of the shape of a reverberation room on the normal modes was examined. Referring to these experiments, the authors made experimental equipment to visualize acoustic resonance phenomena for an educational purpose. In our experiment, two types of two-dimensional boxes with hard surface were prepared. In these boxes, normal modes in a closed sound field and the Helmholtz resonance phenomena, which are essential and important for architectural acoustics, can be visualized. These physical experiments are visually impressive on students in architectural courses and therefore the experiment is efficiently used in architectural acoustic courses.

2aED2. Animations of different sound field decomposition methods. U. Peter Svensson and Bård Støfringsdal (Acoust. Group, Dept. of Electron. and Telecommunications, Norwegian Univ. of Sci. and Technol., NO-7491 Trondheim, Norway, svensson@iet.ntnu.no)

Many methods for solving sound-wave propagation problems are based on a decomposition of the sound field. Here, several such decomposition methods will be demonstrated and it will be illustrated how they can give the same final solution even if the partial solutions, or the solutions outside a limited domain, can be very different. Animations of harmonic and transient solutions will be used to illustrate sound-field synthesis based on a decomposition into plane waves, cylindrical harmonics, and geometrical acoustics plus edge-diffraction components. It will also be shown how a sound field that is measured in discrete points can be converted to different representations. These decompositions have pedagogical merits but can also be used in sound-field reproduction techniques. Examples will include free-field situations as well as indoor cases with a multitude of reflections. [This research was supported by the Research Council of Norway.]

2aED3. Introduction of sound material in living environment 2004 (SMILE 2004): A sound source database for educational and practical purposes. Keiji Kawai (Grad. School of Sci. and Technol., Kumamoto Univ. 2-39-1 Kurokami Kumamoto 860-8555, Japan, kawai@arch.kumamoto-u.ac.jp), Kazutoshi Fujimoto (Kyushu Univ.), Teruo Iwase (Niigata Univ.), Tetsuya Sakuma (The Univ. of Tokyo), Yoshito Hidaka (Tohwa Univ.), and Hirohito Yasuoka (The Ctr. for Better Living)

Sound sources are used in various settings of education, research, and business that are related to architectural acoustics. Especially in educational scenarios, they are very effective for students to learn theories together with the experience of listening to actual sounds related to them. Sound Material In Living Environment (SMILE 2004), a sound source database published in 2004 in DVD form (Japanese only), is the culmination of our project. SMILE 2004 is intended as a collection of sample sounds for use in lectures on architectural acoustics at universities or industrial high schools. In addition, it is useful for measurements or demonstrations for academic or practical purposes. The database contains 913 digital sound files of real sounds and impulse responses, which cover the 
study fields of acoustics, noise control, and soundscapes. Each sound is accompanied by such information as the 1/1 octave band sound pressure levels and a brief explanation and photographs of the situation in which the sound was recorded. In addition, sample presentations for lectures and practical demonstrations are attached as examples of the use of this database for educational and practical purposes. Details of all the above features will be introduced in a demonstration.

2aED4. A toy using vibrations. Kentaro Nakamura and Sadayuki Ueha (Precision and Intelligence Lab., Tokyo Inst. of Tech., R2-26, 4259 Nagatsuta, Midori-ku, Yokohama 226-8503, Tokyo, Japan)

This study is focused on a toy using vibrations. The toy is composed of a rectangular wooden bar with ten notches and a thin wooden plate at one end of the bar. The plate has a hole at its center and is loosely put at the bar end using a pin. The plate can rotate freely. If notches on the bar are scratched by another bar, the plate begins to rotate due to the vibrations excited on the rectangular bar. The interesting thing is that the rotation direction can be controlled if the way of rubbing is altered. The operation principle of the toy is revealed through experimental observations. The measurements of vibration locus of the pin help us to understand the rotation mechanism. According to these results, the method to control the rotation direction is explained and the analogy to the operation principle of ultrasonic motors is presented.

2aED5. The multimedia simulator of radiation sound field created by a vibration disc. Kohei Michigami, Takahiro Masuda, Hiroshi Suda, and Yoshiro Miida (Chiba Inst. of Technol., 2-17-1 Tsudanuma, Narashino_city, Chiba 275-0016, Japan, kohei@miida.net.it-chiba.ac.jp)

In the field of acoustic education, acoustic phenomena are represented in textbooks as sound models and complex numerical formulas. For that reason, it is difficult for students to visualize, intuitively, the relations between acoustic phenomena and complex numerical formulas. Sufficient understanding was not obtained using static teaching materials, such as sentences and figures in textbooks and notes on a blackboard. Therefore, we have produced multimedia teaching materials that render difficult phenomena as interactively visible. The multimedia teaching materials that have been produced comprise explanatory slides, relation movies, and simulator teaching materials. They show a radiation sound field created by a vibrating disc simulator. It is necessary to calculate many complex series to compute the sound field. Then, the sound field is changed complexly and remarkably by wavelength. Therefore, it is a difficult field to understand for students. Our multimedia teaching material expresses it using 3D-CG. Students can see details of the sound-field changing perspective and the wavelength and disc size ratio. Moreover, students can use it to verify the sound pressure level of a graphically selected point, just as with a measuring instrument.

2aED6. Technical listening training: Systematic training program designed to improve auditory sensitivity. Kazuhiko Kawahara, Masayuki Takada, Shin-ichiro Iwamiya, Yoshitaka Nakajima (Dept. of Acoust. Design, Faculty of Design, Kyushu Univ., 4-9-1 Shiobaru Minami-ku, Fukuoka, 815-8540, Japan, kawahara@design.kyushu-u.ac.jp), and Kazuo Ueda (Kyushu Univ., Fukuoka, 815-8540, Japan)

Technical listening training is a systematic training program designed to improve auditory sensitivity. Through this program, students can obtain the necessary auditory sensitivity before gaining work experience on the job. The type of auditory sensitivity acquired through experience depends of course on the occupation. In contrast, technical listening training provides wide varieties of experiences, so that students can obtain auditory sensitivities associated with various fields and easily adapt to new auditory environments. Technical listening training was developed at the Department of Acoustic Design of the Kyushu Institute of Design. The program has been improved and is still included as part of the curriculum of the Department of Acoustic Design. The present demonstration exhibits the concept of technical listening training as well as its practical application at the School of Design, Kyushu University.

2aED7. An integrated system for learning musical theories from western classical and popular music. Masanobu Miura (Dept. of Media Informatics, Faculty of Sci. and Technol., Ryukoku Univ., Ostu 520-2194, Japan), Norio Emura, and Masuzo Yanagida (Doshisha Univ., Japan)

Presented here is a support system for learning the theory of harmony, one of the main subjects in music education consisting of a set of forbidden progressions. By solving given tasks in the theory of harmony, students can learn forbidden progressions. Shown here is a system that generates all the allowable solutions for given bass tasks within triads, named "BDS" (Basse Donnée system), together with a checking system for answers given by students, named "VISAGE" (Validity Inspecting System for Answers of Given Exercises). VISAGE is designed to point out note allocations that violate forbidden progressions in an input answer if any. In case an answer of a given exercise does not violate forbidden progressions, the input answer is evaluated from a viewpoint of music. Also presented is "MAESTRO" (Musical Aesthetics Evaluation System for Tonal music in Regular and Orthodox style), which evaluates 
aesthetics of answers to given base tasks. [Work partly supported by Grant-in-Aid for Scientific Research (No. 16700154), MEXT, by High-tech Research Center promoted by Ryukoku University, by Academic Frontier Project promoted by Doshisha University, and by Knowledge Cluster Project promoted by MEXT, Japan.]

2aED8. Acoustic demonstrations for education in speech science. Takayuki Arai (Arai Lab., Dept. of Elec. and Electron. Eng., Sophia Univ., 7-1 Kioi-cho, Chiyoda-ku, Tokyo, 102-8554 Japan)

Acoustic demonstrations are highly effective for education in speech science. We recently developed two educational tools for acoustics. The first educational tool is a set of physical models of the human vocal tract and related models. It contains cylinder and plate-type models [T. Arai, J. Phonetic Soc. Jpn., 5(2), 31-38 (2001)], a sliding three-tube model [T. Arai, Acoust. Sci. Technol., to be published], lung models, an artificial larynx, and head-shaped models [T. Arai, Acoust. Sci. Technol., 27(2), 111-113 (2006)]. Each model has its own advantages and, if combined effectively, can produce a systematic and comprehensive education in speech production from the lungs to the head. The second educational tool is "Digital Pattern Playback (DPP)" [T. Arai et al., Acoust. Sci. Tech., to be published], which converts a spectrographic image of a speech signal back to sound by digital signal processing. A printed spectrogram on a sheet of paper can also be converted immediately after capturing the image from a camera, and we confirmed that this is more intuitive for learners than converting from an electronic image. [Work partially supported by JSPS.KAKENHI (17500603).]

2aED9. Tools for speech perception, production, and training studies: Web-based second language training system, and a speech resynthesis system. Reiko Akahane-Yamada, Takahiro Adachi (ATR, 2-2-2, Hikaridai, Seika-cho, Soraku-gun, Kyoto, 619-0288 Japan), and Hideki Kawahara (Faculty of Systems Eng., Wakayama Univ. 930 Sakaedani, Wakayama, 640-8510 Japan)

We describe a Web-based instruction system for second language (L2) learners, dubbed ATR CALL. This system is a collection of speech perception, production, and comprehension training tools, designed to examine L2 acquisition mechanisms, especially for native speakers of Japanese who are learning English as L2. Each training component focuses on the acoustic-phonetic, prosodic, lexical, or semantic decoding level of spoken language. The stimuli are selected and presented from a large speech database of 13000 English words and 6000 English sentences. In the pronunciation training component, learners' productions are scored using HMMbased evaluation algorithms, and automatic and immediate feedback about pronunciation goodness is provided. Speech analysis tools for language learners are also provided. We also describe a speech manipulation system, dubbed STRAIGHT [Kawahara et al., Speech Commun. (1999)]. This system resynthesizes stimuli with quality as high as the original stimulus even after manipulating multiple parameters. A speech morphing algorithm on this system enables us to manipulate various features independently [Kawahara et al., ICCASP 2003]. Thus, it generates stimulus continuum varying in supra-segmental domain and/or segmental domain. [Work supported by JSPS, and e-Society by MEXT.]

2aED10. Vowel synthesis tool based on transmission line model as a web application. Kohichi Ogata, Takuro Masuya, and Yuichiro Shin (Grad. School of Sci. and Technol., Kumamoto Univ., 2-39-1 Kurokami Kumamoto 860-8555, Japan, ogata@cs.kumamoto-u.ac.jp)

This paper introduces a web application for vowel synthesis in acoustics education. Synthesis systems that provide users with interactive operation for determining vocal tract configuration and vocal fold control parameters can be effective tools for intuitive understanding of vowel production for students. For example, differences in the size of the vocal tract and vocal folds between male and female speakers can be recognized as differences in formant and pitch frequencies in speech sounds. The realization of such a system as a web application is suitable for platform independent use, considering the recent progress in information and communication technology. In our developed system, interactive operation for determining vocal tract configuration and vocal fold control parameters is achieved on a web browser by using a Java applet. These articulatory parameters are transferred to a server computer via a client/server connection using socket programming, and synthetic speech is produced using calculation programs on the server computer. The speech synthesizer installed on the server is based on the hybrid speech production model proposed by Sondhi and Schroeter. A preliminary user test showed that the system demonstrated a certain applicability as a teaching tool.

2aED11. WWW-based multilingualized system for technical listening training. Akira Nishimura (Dept. of Media and Cultural Studies, Tokyo Univ. of Information Sci., 1200-2, Yato-cho, Wakaba-ku, Chiba, Japan)

A WWW-based system for technical listening training is proposed. Messages from the system shown in a WWW browser are multilingualized using the GNU gettext mechanism. A system administrator can easily set up the system in a user's language by translating the message catalog. The WWW-based training system is superior to previous training systems in the following respects: (1) software and hardware can be organized more easily; (2) modification and creation of the contents of training are simple; (3) individual training parameters can be set for users; (4) many users can use the system individually or simultaneously; (5) the results of training for users are saved and summed individually; and (6) difficulty of discrimination training is automatically set up individually. Training experiments of intensity discrimination and frequency discrimination were performed using a prototype of the training system for subjects who were naive to auditory experiments. Significant improvements in the abilities of both intensity and frequency 
discrimination were observed from results of these experiments. The system is guaranteed to be legal for use, distribution, and modification under the terms of the GNU General Public License. Demonstrations and software packages of the system are distributed at http://adlib.rsch.tuis.ac.jp/ akira/atsdemo/index_en.html.

2aED12. Wonders in perception and manipulation of speech. Makio Kashino (NTT Commun. Sci. Labs./ERATO Shimojo Project, JST/Tokyo Inst. Tech., Atsugi, Kanagawa 243-0198, Japan, kashino@avg.brl.ntt.co.jp), Hideki Kawahara (Wakayama Univ., Wakayama, Wakayama 640-8510, Japan), and Hiroshi Riquimaroux (Doshisha Univ., Kyotanabe, Kyoto 610-0321, Japan)

Under certain conditions, the auditory system can display a number of amazing behaviors. Some of these can be quite unique and may have significant importance for our understanding of the perception of sound. This presentation focuses on behaviors closely related to speech perception. One phenomenon that will be described is termed phonemic restoration. In it, sounds actually missing from a speech signal can be synthesized by the auditory system and clearly heard. Another phenomenon involves noise vocoded speech. In it, speech remains highly intelligible despite the fact that much of its spectral detail is absent. Audio demonstrations will be provided so that the phenomena can be directly experienced. Studying these phenomena will help us understand the function of the central auditory system and develop improved techniques for speech recognition and speech synthesis. This presentation will also contain a demonstration of STRAIGHT, a versatile tool allowing manipulation of various aspects of the speech signal. These demonstrations may be of interest not only to students, but also to teachers in physiology, psychology, and engineering.

2aED13. Amazing features of the auditory system. Kaoru Ashihara (AIST Tsukuba Central 6, 1-1-1 Higashi, Tsukuba, Ibaraki 3058566, Japan, ashihara-k@aist.go.jp), Yukio Iwaya (Tohoku Univ., Aoba-ku, Sendai 9808577, Japan), Kengo Ohgushi (Oheyama-cho Goryo, Nishikyo-ku, Kyoto, 6101102 Japan), and Yoshitaka Nakajima (Kyushu Univ., Minami-ku, Fukuoka 8158540, Japan)

Some audio and audiovisual demonstrations will be presented in order to review the unique nature of the auditory system. The demonstrations cover a wide range of topics: the gap transfer illusion, combination tone, interaction between auditory and visual information, and binaural recording and reproduction. The phenomena to be presented are closely related to the key features of hearing, such as the auditory scene analysis, speech perception, perception of the spatial information, and interaction with other modalities. Because these demonstrations are not only attractive but also of some scientific importance, they can be used as teaching materials in education of physics, physiology, psychology, and engineering. Studying these phenomena will give us insights into the functions of the peripheral and central auditory system.

2aED14. Inexpensive demonstration experiments in acoustics. Andrew Morrison (Dept. of Phys., Illinois Wesleyan Univ., P.O. Box 2900, Bloomington, IL 61702-2900) and Thomas Rossing (Stanford Univ., Stanford CA 94305)

We describe and demonstrate simple classroom demonstration experiments in acoustics using inexpensive equipment.

2aED15. Demonstration of wave synthesis and spectral analysis. Uwe J. Hansen (Dept. of Phys., Indiana State Univ., Terre Haute, IN 47809)

Many inexpensive keyboard synthesizers include a flute sound with overtone levels about $30 \mathrm{~dB}$ below the fundamental. Since these wave shapes are nearly perfect sine waves, they are ideally suited to introduce the concept of complex tone synthesis by adding harmonics. Such a keyboard will be available at the station for demonstration purposes and for viewer experimentation. Complex tone synthesis using amplitude control in a Cool-edit program will also be available.

2aED16. Animations, visualizations, and auralizations in architectural acoustics. Ralph T. Muehleisen (Civil and Architectural Eng., Illinois Inst. of Technol., Chicago, IL 60616, muehleisen@iit.edu)

A number of animations, visualizations, and auralizations developed for use in architectural acoustics classes are presented. Animations of plane waves, spherical waves, and standing waves were created using MATLAB software. Visualizations of modes in regular and arbitrary shaped enclosures were created in Comsol Multiphysics software. Auralizations of tonal and broadband noise, hearing loss, transmission loss, and classroom acoustics were created using Adobe Audition software.

\section{Contributed Papers}

2aED17. Sonification of sound: Auditory display for acoustics education. Densil Cabrera, Sam Ferguson, and Robert Maria (Faculty of Architecture, Univ. of Sydney, NSW 2006, Australia)

Sonification is concerned with data representation to the ear using nonspeech audio. While auralization is routinely used in architectural acoustics, the broader processes encompassed by sonification are not so commonly applied, but can enhance the appreciation of acoustical proper- ties of materials and structures, audio signals, and acoustic system measurements. We present examples of sonifications such as $(i)$ auditory graphs of reflection and transmission coefficients and reverberation times; (ii) auditory graphs of percentiles and moments, applied to time-varying sound levels and to magnitude spectra; (iii) conditioning of room impulse responses for sonification using techniques such as time stretching, signal reversal, filtering and auto-convolution/correlation; (iv) sonification of complex frequency response or signal spectra; and $(v)$ applications of the Hilbert transform for audio signal sonification. Compared to graphic or 
numeric representation, such sonifications have the advantage of providing an aural experience of the aspect of sound that is sonified, and so directly convey to a listener something of the meaning of the data.

2aED18. Rainfall estimation using SORFED (SOund Recording For EDucation). Lucas B. Lezamiz (Appl. Phys. Lab., Univ. of Washington, 1013 NE 40th St., Box 355640, Seattle, WA 98105, lucas@apl.washington.edu)

Underwater sound provides an abundance of information about the environment, both natural and man-made. Using an underwater hydrophone and a webcam above the water's surface, the SORFED website gathers and stores ambient noise from Lake Union 24 hours a day, 30 days at a time. Users can explore the sound signatures of different weather events and human activity on the lake by listening to audio clips side by side with video from the webcam. This website was used as the primary tool for teaching high school students in Seattle how to estimate rainfall by listening to underwater noise. Students worked in teams to calibrate underwater noise to rain rate, then used this relationship to estimate rain rate as a function of time on other days based on the noise level recorded in the lake. Total rainfall was then found by integration. Using similar techniques, students can explore other topics including shipping rate estimation, human behavior and activity, signal-to-noise ratio, and concepts in differentiation and integration.

2aED19. Beamforming with multiple acoustic sources in the classroom. Edward Tucholski (Phys. Dept., Chauvenet Hall, U.S. Naval Acad., 572C Holloway Rd., Annapolis, MD 21402, ejtuchol@usna.edu)

Superposition of multiple sources, constructive and destructive interference, and beamforming are common topics in undergraduate acoustics. While the mathematical models of these topics are fairly easily performed by students, particularly in the far field, the learning experience is enhanced with simple visualizations and by actually building the model with speakers and measuring the resulting amplitude at various angles with a microphone. While this experiment is often performed in an anechoic chamber for best agreement between theory and experiment, the apparatus will work in a typical classroom as a demonstration. The uncomfortable tonal noise in the classroom is eliminated if ultrasonic $(30-50-\mathrm{kHz})$ sources are used. Apparatuses to be demonstrated have been used successfully at the U.S. Naval Academy in both majors and basic physics classrooms, where the professional development of every officer requires an understanding of sensor beamforming.

2aED20. A MIDI (musical instrument digital interface) controller using a mat switch operable by a user in a wheelchair. Takuya Niikawa (Dept. of Biomed. Eng., Osaka Electro-Commun. Univ., 1130-70 Kiyotaki Shijo-Nawate, Osaka 575-0063, Japan), Ryosuke Kawachi, Kotaro Minato (Nara Inst. of Sci. and Technol., Nara 630-0192, Japan), Tatsuo Yoshihara, and Naoya Terayama (Osaka Electro-Commun. Univ., Osaka 575-0063, Japan)

In this study, we devised a MIDI controller that a user with severe physical dysfunction was able to operate by depressing a mat switch with an electric wheelchair, and proposed a new method for musical education and therapy employing the system. This system comprises a mat switch resistant to $100 \mathrm{~N}$ of a movement load in a $500 \times 700 \mathrm{~mm}^{2}$ area, an A/D converter, a PC, and a MIDI device. Signal output from the mat switch is converted to MIDI signals by the PC and transmitted to the MIDI device. By pushing down ten mat switches arranged in a matrix pattern, a user can manipulate all elements necessary to control MIDI signals, such as volume and musical sounds. As a result, it was confirmed that a user in an electric wheelchair was able to control musical notes, volume, tunes, and scales.
Furthermore, since it was possible to use it for a hand bell and a musical game mimicking the card game "Concentration," it may be applicable for musical education.

2aED21. An online virtual acoustic guitar laboratory. Nelson Lee and Julius O. SmithIII (Ctr. for Comput. Res. in Music and Acoust. (CCRMA), The Knoll, 660 Lomita, Stanford, CA 94305-8180, nalee@stanford.edu)

We present a "virtual acoustic guitar laboratory" in which students can bring in their own acoustic guitars, record several notes, and follow a step-by-step procedure to create their own real-time computational model, suitable for use in a synth plugin in an electronic music studio. The guitar synthesis model is based on the commuted waveguide synthesis technique (http://ccrma.stanford.edu/ jos/cs.html). Online laboratory materials are presently available at (http://ccrma.stanford.edu/ jos), the project website (http://ccrma.stanford.edu/realsimple/), and are also being ported to Connexions (http://www.cnx.org/), an online repository of educational materials freely distributable under a Creative Commons license. Connexion's "modules" and "courses" (which are simply module sequences) are readily and freely accessible and modifiable to allow instructors to adapt the materials as appropriate for their own classroom needs. The online lab materials contain Web pointers to relevant portions of online textbooks covering theoretical aspects of the analysis and synthesis methods used, thereby offering the student a demand-driven deeper understanding within a motivating context. [This work was supported by the Wallenberg Global Learning Network.]

2aED22. Application of speech recognition to teaching English as a foreign language. Hiroyuki Obari (Aoyama Gakuin Univ., 4-4-25, Shibuya-ku, Shibuya-ku, Tokyo, 150-8366 Japan), Hiroaki Kojima, Hiroshi Oomura (Adv. Industrial Sci. and Technol., Tsukuba, Ibaraki, 305-8568 Japan), and Machi Okumura (PRONTEST, Tsukuba, Ibaraki, 305-8568 Japan)

English is considered to be an important common international language; therefore, in Japan English education plays an important role to make Japanese speakers of English intelligible enough to be properly understood for international communication. Intonation and the rhythm of the language and pronunciation of each word are crucial to comprehensive speech. So far the CALL system has not been well equipped with effective software for improving pronunciation. This is why PRONTEST and AIST (National Institute of Advanced Industrial Sciences and Technology) have been jointly developing special software for teaching English pronunciation using the technology of Speech Recognition System integrated into the CALL system. This software can automatically correct the crucial pronunciation of lower Japanese EFL learners and teach them how to improve its pronunciation with a good piece of advice after they pronounce several words with the computer. So far we have successfully developed the CALL system, in which we can deal with the following sounds: [r] [1] [s] [sh] [th] [z] [f] [v] [d] [b]. We carried out an experiment and found out that about $90 \%$ of the students were satisfied with correcting their pronunciation using this software at Aoyama Gakuin University.

2aED23. Manuscript preparation with LyXforJASA. John C. Burgess (Dept. of Mech. Eng., Univ. of Hawaii, 2540 Dole St., Honolulu, HI 96822)

LyXforJASA is a graphical software package for creating manuscripts that meet the submission requirements of the Peer-X-Press (PXP) system used by the Journal of the Acoustical Society of America (JASA). Its main features are graphical templates for superscript and author-year citation styles and for single figures. It is based on LyX, a screen-based graphical user interface (GUI) to the LaTeX typesetting language. Authors see manuscript content, not LaTeX code. LyX liberates authors from document bookkeeping, such as spacing, font selection, and section, equation, 
figure, table, and citation numbering. LyX has a comprehensive, point and click, GUI mathematics editor with an extensive list of symbols. It can export text in LaTeX, PDF, PostScript, and ASCII formats. It has been ported successfully to Linux, Macintosh OSX, and Windows XP. LyXforJASA packages can be found at http://ww2.hawaii.edu/ $\sim$ jcb/LyXforJASA/LyXforJASA.html

WEDNESDAY MORNING, 29 NOVEMBER 2006

MONARCH ROOM (ROYAL HAWAIIAN), 8:20 TO 11:35 A.M.

\title{
Session 2aMU
}

\section{Musical Acoustics: Comparing Asian and Western Instruments I}

\author{
Tomoyasu Taguti, Cochair \\ Konan Univ., 8-9-1 Okamoto, Higashi-ku, Kobe 658-8501, Japan \\ Thomas D. Rossing, Cochair \\ Stanford Univ., CCRMA, Dept. of Music, Stanford, CA 94305
}

Invited Papers

8:20

2aMU1. Acoustics of Eastern and Western bells, gongs, and drums. Thomas Rossing (CCRMA Dept. of Music, Stanford Univ., Stanford, CA 94305)

Bells, gongs, and drums have been part of most Eastern and Western cultures throughout history. We compare the acoustics of some examples of these types of percussion instruments, both contemporary and historical.

8:40

2aMU2. Acoustics of the Korean percussion instruments pyeongyeong and pyeonjong. Junehee Yoo (Dept. of Phys. Education, Seoul Natl. Univ., Seoul 151-742, Republic of Korea, yoo@snu.ac.kr) and Thomas D. Rossing (Stanford Univ., Stanford, CA 94305)

Pyeongyeong is a set of $16 \mathrm{~L}$-shaped chime stones and pyeonjong is a set of 16 oval chime bells. Vibrational mode shapes and mode frequencies of the pyeongyeong determined by holographic interferometry and by experimental modal testing agree very well with those calculated using finite-element methods. A model of the pyeongyeong in which the geometry can be varied shows that the actual shape is probably the one that gave the preferred sound. Vibrational mode shapes and frequencies of the pyeonjong, also determined by holographic interferometry and by experimental modal testing, are split into doublets due to the oval shape of the bells. The splitting is less than in Chinese two-tone bells but it gives rise to audible beats, as in large Korean temple bells.

\section{9:00}

2aMU3. Acoustics of the Tibetan long trumpet. Murray Campbell (School of Phys., Univ. of Edinburgh, Edinburgh EH9 3JZ, UK, d.m.campbell@ed.ac.uk) and Arnold Myers (Univ. of Edinburgh, Edinburgh EH8 9AG, UK)

The Tibetan long trumpet, or dung-chen, is a metal horn made in several telescoping conical sections. Dung-chen range in length from around 1.3 to over $3 \mathrm{~m}$; the integral mouthpiece is wide and very shallow. Identical pairs of dung-chen are used in rituals of Tibetan Buddhism, often in combination with other instruments. This paper describes bore profile and input impedance measurements on a number of examples of the dung-chen, and compares the acoustical and musical properties of these Asian brass instruments with those of Western European long trumpets and horns.

9:20

2aMU4. Sound production in Asian and Western free-reed instruments. James P. Cottingham (Phys. Dept., Coe College, Cedar Rapids, IA 52402)

There are two families of free-reed instruments. This paper summarizes some of the research on both done in recent years, with emphasis on differences in history and means of sound production. The Asian mouth-blown instruments are of ancient origin and use a free reed coupled to a pipe resonator. The sheng, sho, khaen, and bawu are typical examples. These reeds are approximately symmetric, typically cut from a single strip of material, and often operate on both directions of air flow. They behave as blown-open or outward-striking reeds, with playing frequency above both the resonant frequency of the pipe and the natural frequency of the reed. The Western free-reed family originated in Europe about 200 years ago and includes the reed organ, harmonium, harmonica, and the accordion-concertina family. In these the reed tongue is offset from the opening in the frame. This permits operation on only one direction of air flow, and pipe resonators are not required and generally not used. The playing frequency of the air-driven reeds is normally below the natural reed frequency and, if the reed is coupled with a pipe, below the resonant frequency of the pipe as well. 
2aMU5. Comparing the Hawaiian nose flute with the western concert flute. Lydia Ayers and Andrew Horner (Hong Kong Univ. of Sci. and Technol., Clear Water Bay, Kowloon, Hong Kong)

This presentation will compare the spectral and expressive characteristics of the Hawaiian nose flute with the western concert flute. The Hawaiian nose flute is a bamboo courtship flute. This presentation will give live demonstrations on several nose flutes and the western flute, along with brief descriptions of the musical and spectral characteristics of these instruments, and then describe the additive synthesis design template used to capture their subtle timbral characteristics using score data in Csound. In addition, the expressive design successfully uses spectral interpolation for the idiomatic ornamental phrasing for each instrument. A special amplitude/frequency function modulation method simulates the trills, which are also slurred within phrases of varying numbers of notes. [This work was supported by the RGC Competitive Earmarked Research Grant 613505, with special thanks to Rodney "Kala" Willis for making the nose flutes.]

\section{0:00-10:15 Break}

\section{0:15}

2aMU6. Cross-cultural comparison of flute acoustics and playing techniques. Peter L. Hoekje (Dept. of Phys. and Astron., Baldwin-Wallace College, Berea, OH 44017, phoekje@bw.edu) and Pedro Eustache (Castaic, CA 91384)

Examples of flutes from different cultures around the world will be demonstrated, including European flutes, Persian ney, Egyptian kawala, traditional Indian bansuri, custom King bansuri, Japanese shakuhachi, Chinese di, South American kena, and Polynesian nose flutes. The differences among these may in large part be classified according to the details of their sound production mechanism at the mouthpiece, and of the bore and toneholes that form the resonator. These features are closely linked with the sound of the instrument as well as the notes and music played on it, and the playing techniques and styles developed for it. A cross-cultural problem arises when a musician needs to play with the sound and style of a non-Western flute tradition in the context of Western instruments and music. Various solutions to this problem, involving adaptation of either the mouthpiece or the resonator, have been developed.

\section{Contributed Papers}

\section{0:35}

2aMU7. Tunings of historical Korean pyeongyeong and pyeonjong. Junehee Yoo, Suyoung Jin, Hyunsoo Kim (Dept. of Phys. Education, Seoul Natl. Univ., Seoul 151-742, Republic of Korea, yoo@snu.ac.kr), Koeng-mo Sung, Junguk Noh, and Hyun-woo Ko (Seoul Natl. Univ., Seoul 151-742, Republic of Korea)

In order to reconstruct the whangjongeum or scale in Korean traditional instruments, we have measured frequencies of historical pyeongyeong stones and pyeonjong bells mainly from the 14th to 19th centuries. Pyeongyeong consists of 16 chime stones tuned to a scale quite similar to the Western scale beginning with $\mathrm{C} 4$. Pyeongjong consists of 16 chime bells, tuned approximately an octave lower than pyeongyeong. We present frequency averages and standard deviations for 124 chime stones and 64 chime bells available in the National Center for Korean Traditional Performing Arts. The spread in frequencies appears to be greater in some periods in history than in others, but the average frequency is quite consistent.
10:50

2aMU8. Input impedance of asian free-reed mouth organs. Eric A. Dieckman (Truman State Univ., Kirksville, MO 63501) and James P. Cottingham (Coe College, Cedar Rapids, IA 52402)

The khaen, naw, bawu, and sheng are examples of Asian free-reed mouth organs, which incorporate free reeds coupled to pipe resonators. Previous research has shown that the reeds in these instruments behave as blown-open reeds: the playing frequency is above both the natural frequency of the reed and the first peak of the measured impedance curve. Detailed calculations of input impedance have been made for a variety of these instruments, taking into account the position of the reed along the pipe, tuning slots, finger holes, and noncircular cross sections. The details of these calculations are in good agreement with the measured impedances of the same instruments, even in the higher harmonics. A study of the coupling of the free reed and pipe resonator has provided additional details about the influence of the pipe impedance on the playing frequency and sound spectrum of the reed-pipe combination. [Research supported by NSF REU Grant PHY-0354058.]

11:05-11:35

Mini-concert of flute music by Pedro Eustache 


\title{
Session 2aPA
}

\section{Physical Acoustics and Noise: Sonic Boom From Supersonic Aircraft and Projectiles I}

\author{
Victor W. Sparrow, Cochair \\ Pennsylvania State Univ., Graduate Program in Acoustics, 316B Leonhard Bldg., University Park, PA 16802 \\ Kazuyoshi Takayama, Cochair \\ Tohoku Univ., Biomedical Engineering Research Organization, 2-1-1 Katahira, Aoba, Sendai 980-8577, Japan
}

Chair's Introduction-8:00

Invited Papers

8:05

2aPA1. Model experiments to study sonic boom propagation through turbulence. Philippe Blanc-Benon and Sebastien Ollivier (LMFA, UMR CNRS 5509, Ecole Centrale de Lyon, 69134 Ecully Cedex, France)

The aim of this paper is to present data from laboratory-scale experimental simulations of sonic boom propagation through a turbulent atmosphere. The source used to simulate sonic boom $\mathrm{N}$ waves is an electric spark source. In order to model turbulence, two setups have been used: a turbulent jet for kinematic turbulence and a heated grid for thermal turbulence. Acoustic measurements have been conducted for three configurations: free field; close to a plane surface in order to simulate ground effects; and close to a curved surface in order to investigate the propagation into a shadow zone. The surfaces were either smooth or rough. With turbulence, many waveforms have been recorded, then the statistics of various parameters, including the peak pressure and the rise time, have been analyzed. In the free field, data show that the increase of the mean rise time and the decrease of the mean peak pressure are linked to the probability of random focusing. The experiments have enabled separate assessment of the effects of turbulence and roughness in the shadow zone caused by the curved surface. Comparison with numerical simulations will be discussed. [This work was supported by European Commission Contract N:G4RD-CT-2000-00398 and French Ministere de la Recherche (Decision 00T0116).]

8:25

2aPA2. Sonic-boom research activities in the Japan Aerospace Exploration Agency (JAXA). Yoshikazu Makino (JAXA, 7-44-1 Jindaijihigashi-machi, Chofu, Tokyo 182-8522, Japan, makino.yoshikazu@jaxa.jp)

Some sonic-boom research activities conducted in the Japan Aerospace Exploration Agency (JAXA) are summarized. JAXA started a scaled supersonic experimental airplane program named NEXST-1 in 1996 in order to establish advanced CFD-based design technologies. The flight test of the NEXST-1 was conducted in October, 2005 at Woomera test range in Australia. The sonic-boom signatures of the NEXST-1 were measured on the ground in order to confirm the possibility of demostrating the low sonic-boom design technology with scaled experimental airplanes for a future low-boom demonstrator program. The measured sonic-boom signatures were compared to the signatures extrapolated from the near-field pressure signatures predicted by CFD and panel method or measured in the supersonic wind tunnel. Low-sonic-boom design technology using gradient-based optimization tool with CFD is developed and applied to a non-axisymmetrical fuselage design concept in order to reduce the drag penalty. JAXA works on a human acceptability study in cooperation with Kawasaki Heavy Industries (KHI) using their sonic-boom simulator. Some low-boom signatures in addition to some typical $\mathrm{N}$-shaped signatures with various peak pressure levels were simulated in the boom box and used for subjective test in order to collect the human acceptability data for future sonic-boom acceptability criteria.

\section{8:45}

2aPA3. Prediction and measurement of a weak sonic boom from an entry vehicle. Kenneth J. Plotkin (Wyle Labs., 2001 Jefferson Davis Hwy., Ste. 701, Arlington, VA 22202, kenneth.plotkin@wylelabs.com), Russell J. Franz, and Edward A. Haering, Jr. (NASA Dryden Flight Res. Ctr., Edwards, CA 93523)

There is a current interest in measuring low-amplitude sonic booms in the atmosphere. This interest is related to verifying the predicted loudness and structure of the shock waves. The reentry of the Stardust comet sample probe on 15 January 2006 provided an opportunity for such a measurement. PCBoom4 was used, together with Stardust's projected reentry trajectory, to predict the sonic boom footprint. A theory for the sonic boom of a drag-dominated blunt hypersonic vehicle had been developed by Tiegermann [Ph.D. thesis, Cornell University, 1975]. This theory provides an effective $F$-function based on energy dissipation, using a blast wave analogy and numeric solution. The inputs to that model are the vehicle drag and flight conditions. Tiegermann's model was implemented in PCBoom4. Drag was obtained by computing deceleration from the expected entry trajectory and multiplying by vehicle weight. A boom with peak overpressure of 0.0524 psf, arriving at 10:00:28.465 UTC, was predicted for a planned measurement site in Ruby Valley, NV, using an atmospheric profile from the upper air reporting station at nearby Elko, NV. The measured sonic boom had a peak overpressure of $0.052 \mathrm{psf}$ and arrived at 10:00:29.719 UTC. 
2aPA4. Toward silent supersonic transport-A fundamental study of supersonic biplane. Kazuhiro Kusunose (Tech. Res. and Development Inst., Japan Defense Agency, Sakae-cho 1-2-10, Tachikawa, Japan 190-8533), Daigo Maruyama, Kisa Matsushima, Kazuhiro Nakahashi (Tohoku Univ., Sendai 980-8579, Japan), Hiroshi Yamashita, Masahito Yonezawa, and Shigeru Obayashi (Tohoku Univ., Sendai, Japan 980-8577)

In this research, aerodynamic design of biplane airfoils in supersonic flight is discussed based on computational fluid dynamics (CFD). In supersonic flight, airfoils generate strong sonic booms and wave drags accompanied by shock waves. New airfoil geometries which significantly reduce shock waves using a biplane concept will be proposed. The background of this concept originates from Busemann biplane and Licher type biplane concepts. In order to focus on the shock-wave characteristics around biplane configuration, inviscid flow (Euler) analyses are performed (which are particularly suitable for wave drag analyses). For the evaluation of the reduction level of shock waves, the wave drag coefficient is used. The design Mach number is 1.7. The aerodynamic design is conducted using an iterative inverse design method that is newly implemented. A biplane configuration with a desired performance has been obtained. Having total maximum thickness ratio of 0.10, it has the lift-to-wave-drag ratio of 21.7 at a desired lift condition for supersonic flight. At the range of lift coefficient more than 0.14 this designed biplane has lower wave drag than that of a (zerothickness) single flat plate airfoil. This result will lead to the realization of silent supersonic transport.

9:25

2aPA5. Ballistic range simulation of sonic boom generation. Kazuyoshi Takayama (Biomed. Eng. Res. Organization, Tohoku Univ., Sendai, 980-8577, Japan), Ming-Yu Sun, and Takamasa Kikuchi (Tohoku Univ., Sendai, 980-8579, Japan)

A result of recent ballistic range experiments applied to a sonic boom simulation is reported. For flight simulations at transonic speed ranging from 340 to $400 \mathrm{~m} / \mathrm{s}$, we used a 51-mm bore gas gun launching 51-mm-diam sabot and projectile combinations, and for supersonic flight simulations exceeding $400 \mathrm{~m} / \mathrm{s}$ we used a $15-\mathrm{mm}$ bore two-stage light gas gun. Flow diagnostics were performed mainly by double-exposure holographic interferometry, shadowgraph recorded with a high-speed video camera, and pressure measurements. The present experimental setup is so versatile that we placed a water basin on the recovery tank floor and measured interactions between sonic booms and wavelets in the water basin. As one of the basic research topics related between shock dynamics and aeronautics, we launched 40-mm-diam spheres at high transonic speed of shock Mach number ranging from 1.01 to 1.1 at increments of every 0.01 , visualized quantitatively flows over spheres, and could determine shock standoff distances over spheres. The speed range we simulated is in the region hardly achievable in existing wind tunnels.

\section{9:45}

2aPA6. Atmospheric turbulence filter functions derived from high-fidelity measurements. Lance Locey and Victor Sparrow (The Pennyslvania State Univ. Grad. Program in Acoust., State College, PA 16803)

Efforts have been underway to develop filter functions suitable for adding turbulent atmospheric effects to theoretical low-boom waveforms. Filter functions have been created based on a measured input-output relationship. The input is a relatively clean sonic boom waveform measured at altitude by a glider, and the outputs are turbulized' waveforms measured on the ground. One input waveform and multiple output waveforms are used to represent multiple realizations of the atmosphere. Work presented in 2005 [Locey and Sparrow, Innovations in Nonlinear Acoustics, 17th International Symposium on Nonlinear Acoustics (American Institute of Physics, Melville, NY, 2006)] yielded an initial set of filter functions using one particular algorithm and data collected during the Shaped Sonic Boom Experiment (SSBE) in January of 2004. In this talk new results will be presented based on high-fidelity measurements made at NASA Dryden Flight Research Center in June of 2006 [T. Gabrielson et al., Proc. Internoise (2006)]. Time permitting, additional methods for obtaining filter functions will be discussed, including the use of existing sonic boom propagation codes modified to include atmospheric turbulence. [Work supported by the FAA/NASA/Transport-Canada PARTNER Center of Excellence for Aircraft Noise and Aviation Emissions Mitigation.]

\section{0:05-10:20 Break}

\section{0:20}

2aPA7. Atmospheric effects on sonic boom loudness. Anthony R. Pilon (Adv. Development Programs, Lockheed Martin Aeronautics Co., 1011 Lockheed Way, Palmdale, CA 93599, tony.pilon@1mco.com)

The effects of sonic boom propagation through nonstandard atmospheres have been studied. Variations in atmospheric temperature, pressure, humidity, and winds can have significant impact on the structure of the shock waves in the boom signal, as well as the propagation path of the boom. In the study, a hybrid time-frequency domain code was used to calculate finely resolved sonic boom signals for a variety of atmospheric conditions. Idealized near-field signals for low amplitude "N-wave" and "shaped" booms were used to calculate ground level signals. The perceived level metric of Stevens ["Perceived level of noise by Mark VII and decibels (E)," J. Acoust. Soc. Am. 51, 575-601 (1972)] was used to quantify the loudness of the calculated booms. Results of the study will be used to determine the impact of atmospheric variations on the acceptability of sonic booms from future supersonic cruise aircraft.

\section{0:40}

2aPA8. Discrete-time modeling of atmospheric turbulence with application to sonic booms. Joe Salamone (Gulfstream Aerosp. Corp., P.O. Box 2206 M/S R-01, Savannah, GA 31402-2206, joe.salamone@gulfstream.com)

A method is presented that computes a discrete-time model of the atmospheric turbulence effects on sonic booms. First, signal processing techniques are applied to sonic booms to extract the shock profiles from $N$ waves measured on the ground. Next, clean shock profiles are estimated from nonlinear acoustics theory using the measured sonic boom ground data as a reference. A discrete- 
time model is computed using a Wiener filtering scheme with the "clean" shock profile as the input to the scheme and the measured shock data as the output. The resulting system of equations required to compute a model is ill conditioned. Tikhonov regularization is implemented with the regularization parameter chosen based on inspection of the eigenvalue spectrum of the autocorrelation matrix. Multiple models are obtained for "low" to "high" amounts of turbulence from ground measurements recorded under various atmospheric conditions. Predicted $N$ waves and shaped sonic booms are digitally filtered by the turbulence models. The loudness levels of the sonic booms before and after application of the turbulence models are evaluated.

\section{1:00}

2aPA9. Extension of a sonic boom model to ellipsoidal Earth and 3-D atmosphere. Kenneth J. Plotkin (Wyle Labs., 2001 Jefferson Davis Hwy., Ste. 701, Arlington, VA 22202, kenneth.plotkin@wylelabs.com) and Edward A. Haering, Jr. (NASA Dryden Flight Res. Ctr., Edwards, CA 93523-0273)

Sonic boom ray tracing models generally use flat-earth geometry, with 3-D ray propagation through a horizontally stratified atmosphere. There has been recent interest in long-range sonic boom propagation, both for analysis of sonic boom carpet edges from high-altitude flight and for analysis of over-the-top booms. The sonic boom program PCBoom4 has been extended to incorporate ellipsoidal earth and full 3-D ray tracing through a three-dimensional atmosphere. Ellipsoidal geometry of the Earth is handled by the method of Sofair [J. Guidance Control, 23(2)], which defines a local vertical at given coordinates. A tangent reference plane is defined, and ray tracing is performed using the 3-D formulation of Schulten [NLR TP 97-374], in Cartesian coordinates. Once rays are traced, ray tube areas, signature aging, and focal zones are computed in the traditional manner. When over the top booms are analyzed, a starting signature for propagation after the caustic passage is generated from the recent numeric calculations by Kandil [AIAA Paper No. 2005-0013]. The new program, denoted PCBoom5, is expected to be available in 2007.

11:20

2aPA10. Supersonic aircraft: Shake, rattle and role. Sandy Liu (Federal Aviation Admin., Office of Environment and Energy, (AEE-100) Noise Div., Washington, DC)

There has been a renewed interest worldwide to focus on identifying technological advances that could satisfy the production of a new commercial supersonic aircraft. Although advances have been realized since the Concorde was developed, there is widespread agreement that many challenges remain. At a lesser risk, a supersonic business jet (SBJ) is seen as a technological stepping-stone in the overall tactic of advancing the speed of commercial aircraft. This presentation outlines some of the technological and environmental regulatory challenges facing such an aircraft. This includes a review of the established technical noise working group and activities that address supersonic aircraft environmental matters: sonic boom, aircraft community noise, and aircraft emissions. Federal Aviation Administration (FAA) maintains that a successful supersonic aircraft will depend on its stakeholders' ability to deal with these key issues. FAA finds it beneficial to work within the international arena for addressing these issues to ensure that all stakeholders' concerns are considered. Ultimately, successfully addressing concerns regarding sonic boom is a necessary initial condition for regulatory authorities to reconsider existing restrictions on supersonic operations, the key constraint to the business case in the revival to develop a civil supersonic aircraft for the future.

11:40

2aPA11. Flight demonstration project for low-sonic boom design technology. Akira Murakami (6-13-1 Osawa, Mitaka, Tokyo, 181-0015 Japan)

Japan Aerospace Exploration Agency (JAXA) has promoted a supersonic technology research program including flight demonstration since 1997. In this program, JAXA is now planning the new flight demonstration project "Silent Supersonic Technology Demonstrator Project (S3TD)." One of the targets of this project is to demonstrate the low sonic boom aircraft concept to reduce sonic boom intensity by $50 \%$ as compared with conventional configuration and to modify the sonic boom signature for not only front shock boom but also the rear shock one. JAXA will develop a new flight demonstrator that is an unmanned vehicle of approximately 3 tons in weight and $13 \mathrm{~m}$ in length, and then JAXA will make flight trials by 2012. Now JAXA is designing the base-line configuration, using the CAD-based Automatic Panel Analysis System that we have developed. The shock overpressure of the S3TD base-line configuration is estimated to be about $0.23 \mathrm{psf}$ for front shock and $0.21 \mathrm{psf}$ for rear shock, corresponding to about $50 \%$ intensity as compared with those of $N$-wave signature estimated by the first-cut method. The robustness of the low boom signature is also considered. The rear shock boom signature is so sensitive to flight condition that some small device is installed around the aft deck. 


\title{
Session 2aPP
}

\section{Psychological and Physiological Acoustics: Spatial Hearing and Temporal Processing (Poster Session)}

\author{
Eric W. Healy, Cochair \\ Univ. of South Carolina, Dept. of Communication Sciences and Disorders, Columbia, SC 29208 \\ Masashi Unoki, Cochair \\ JAIST, School of Information and Science, 1-1 Asahidai, Nomi, Ishikawa 923-1292, Japan
}

\section{Contributed Papers}

\begin{abstract}
All posters will be on display from 8:30 a.m. to 12:00 noon. To allow contributors an opportunity to see other posters, contributors of odd-numbered papers will be at their posters from 8:30 a.m. to 10:15 a.m. and contributors of even-numbered papers will be at their posters from 10:15 a.m. to 12:00 noon.
\end{abstract}

2aPP1. Motion perception of the target sound event under the discriminated two sound events. Tsuruyo Nishida (Gifu Natl. College of Tech., Motosu, Gifu 501-0495, Japan), Kazuhiko Kakehi, and Takamasa Kyutoku (Chukyo Univ., Toyota, Aichi 470-0393, Japan)

The correct percentage of motion direction for one sound event was about $90 \%$. In the case of two sound events, the correct percentage was lower than for one sound event. The sound event was presented through the headphone in these experiments. These two sound events were physically separated both in frequency and in onset time for the two sound events. It might be possible to perceive two sound events separately. The perception of onset time difference and the discrimination of different frequency sound events were investigated to clarify whether the subjects perceived two sound events distinctly. When IEOI (interevent onset interval) was more than $40 \mathrm{~ms}$, the subjects perceived the onset time difference and the order of two presented sound events. Results clarified that the subjects distinctly perceive two sound events separately with long IEOI. However, the correct percentage for the direction of motion perception of the target in two sound events was significantly lower than for one sound event. The target was strongly affected by the nontarget. Even when two sound events are perceived separately in terms of frequency and temporal order, it is difficult to perceive the motion direction of the target.

2aPP2. Perceptual movement of sounds moved up or down through multiway loudspeakers set up perpendicularly. Yu Agastuma and Eiichi Miyasaka (Musashi Inst. of Technol., 3-3-1 Ushikubo-Nishi, Tsuzuki-ku, Yokohama, Japan)

Perceptual movements of sounds fed through eight loudspeakers perpendicularly set up were investigated. The loudspeakers, which were mutually separated by $30 \mathrm{~cm}$, were set up immediately in front of 15 observers positioned $4.5 \mathrm{~m}$ distant from the loudspeakers. They were required to answer the start point of the loudspeaker through which they perceived that the sound was output at the start, and the end point of that loudspeaker. The results show that the ascension or descension of the sound images was perceived when $\frac{1}{3}$ octave bands of noises with center frequencies of 1,4 , and $8 \mathrm{kHz}$ were linearly moved up or down in the range from 60 to $150 \mathrm{~cm}$. The results also show that the perceived moving distance depends on the moving speed, becoming longer than the physical distance at speeds of 10 and $20 \mathrm{~cm} / \mathrm{s}$, and converging to the physical distance concomitant with higher moving speed, irrespective of the center frequencies. [Work supported by SCAT.]
2aPP3. Combined manipulations of the perceived location and spatial extent of the speech-target image predominantly affect speech-onspeech masking. Ying Huang, Xihong Wu, Qiang Huang, and Liang Li (Speech and Hearing Res. Ctr., Peking Univ., Beijing, China, 100871, liangli@pku.edu.cn)

Speech maskers contain both informational-masking and energeticmasking components. To fully understand speech masking, it is critical to separate these two types of masking components. This study investigated the effect of the inter-target-source delay (ITSD) on intelligibility of speech when both the speech target and masker were presented by each of the two spatially separated loudspeakers (located at -45 and +45 degrees, respectively). The masker was either two-voice speech (different contents between the two loudspeakers) or steady-state speech-spectrumnoise (uncorrelated between the two loudspeakers). The results show that as the ITSD was decreased from 64 to $0 \mathrm{~ms}$, the target image progressively became funneled into the region around the midline, and the intelligibility of the target was monotonically improved by over $40 \%$ when the masker was speech, but by only about $10 \%$ when the masker was noise. Under the quiet condition, however, the intelligibility was not affected by the change of ITSD. The results suggest that combined manipulations of perceived location and spatial extent of the speech-target image by changing the ITSD predominantly affect informational masking of speech. [Work supported by China NSF and Canadian IHR.]

2aPP4. Free-field sound localization with nonlinear hearing protection devices. Kimberly B. Lukas and William A. Ahroon (U.S. Army Aeromedical Res. Lab., Fort Rucker, AL 3636,)

Hearing enhancement and protection devices are being developed for both civilian and military markets that permit the wearer to maintain an awareness of the ambient sound environment and permit face-to-face communications while still protecting the user from high-level impulse noises or blast waves from, for example, handguns, rifles, and shotguns. The impulse noise protection for these devices can range from less than $9 \mathrm{~dB}$ peak pressure reduction to reductions of more than $40 \mathrm{~dB}$ peak. There has been little research, however, on the ability to use binaural cues to localize sound with such devices. An experiment to evaluate the ability of normalhearing listeners to localize broadband noise bursts while wearing various nonlinear hearing enhancement and protection systems was conducted in an anechoic environment. Subjects were asked to localize a 60-dB SPL, 200-ms broadband noise burst from a speaker in 1 of 12 different azimuth locations ( 0 to 330 deg in 30-deg steps) and five different elevations ( 0 , 
$\pm 15, \pm 30 \mathrm{deg}$ ). The azimuth and elevation errors were measured for each device using circular statistical techniques. Front/back reversals were corrected. The results show a 15- to 20-deg degradation in the wearer's ability to localize sound when using these hearing protectors.

2aPP5. Selection of attractor sounds for an audio-based navigation system for the visually impaired. Brad S. Salisbury, Koorosh Naghshineh (Western Michigan Univ., Kalamazoo, MI 49008-5343), and William Wiener (Marquette Univ., Milwaukee, WI 53233)

Research into human hearing has been very laboratory oriented in the sense that the test environments do not replicate most real world situations [J. C. Middlebrooks and D. M. Green, Annu. Rev. Psychol. 42, 135-159 (1991)]. While very useful information is gained from these types of tests, it is difficult to see how real world situations affect sound source localization, recognition, and navigation (walking/way finding) performance. Such information is especially important to people who are visually impaired and dependent on prior knowledge of the environment or audio cues for travel. The research reported here was conducted during the development of an audio-based navigation system. The question that arose was, what constitutes good attractor sounds versus bad sounds? A series of physical tests was developed to identify sounds that performed best from within a group of real-world attractor sounds. Testing was conducted with the aid of participants who were blind or visually impaired. Results of the experiments were consistent with those of Landau et al. [Asst. Technol. 17, $133-143$ (2005)].

2aPP6. A study of the equivalent perception between a visual stimulus with a feeling of depth and its associated sound. Hiroshi Hasegawa, Akimasa Suzuki, Ichiro Yuyama (Faculty of Eng., Utsunomiya Univ., 7-1-2 Yoto, Utsunomiya-shi, Tochigi-ken 321-8585, Japan), Hirotaka Ono, Masao Kasuga, and Miyoshi Ayama (Utsunomiya Univ., Utsunomiya-shi, Tochigi-ken 321-8585, Japan)

This study investigated the equivalent perception between a visual stimulus and its associated sound. Auditory-visual stimulus presentation experiments were performed using an audio-video clip of a man beating a drum on a road. The visual stimulus, with a perspective view of the road, imparted a depth sensation. Visual stimuli were projected onto a screen of 30.8 (W) (B 16.1 (H) deg. In all, 315 auditory-visual stimuli were produced with combinations of distance between the visual target (the drum) and the observer from (B00 m, the time delay between auditory and visual stimuli from $-8 \mathrm{~F}$ to $8 \mathrm{~F}\left(1 \mathrm{~F}=\frac{1}{30} \mathrm{~s}\right)$, and the sound pressure level of the sound stimulus from $(\mathrm{B} 12 \mathrm{~dB}$. The stimuli were presented to six subjects. In each presentation pattern, the sound pressure level to provide a perceptual strength equivalent to that of the visual target was determined: the equivalent sound pressure level (ESPL). Results show that the ESPL increased as the time delay between the auditory and visual stimuli increased, i.e., the ESPL tended to shift in the direction that the event was closer to the observer. The time delay shifted in the direction that the event was distant from the observer.

2aPP7. Localization of text-to-speech voices in a virtual speech display. Gordon Rubin (AT\&T Labs, Florham Park, NJ 07932, and Rensselaer Polytechnic Inst., Troy, NY 12180)

An auditory display using high-quality text-to-speech (TTS) voices is presented to a group of individuals. Subjects are asked for their impression of the source direction of a synthesized talker in a nonreverberant environment. Location is coded using a set of nonindividualized head-related transfer functions (HRTFs). Many years of research in binaural technology provide comparative data for the localization ambiguities inherent in such presentation (e.g., front-back confusion). These ambiguities are characterized for stimuli generated by a mature concatenative speech synthesis system. An initial attempt is made to quantify the perceptual significance realized by a binaural-model-motivated speech synthesizer.
2aPP8. Using localization cues to track one of two concurrent sound sequences. Marie Rivenez, Anne Guillaume, Lionel Pellieux, and Christelle Delor (Departement Sci. Cognit. 5 IMASSA, BP 73, 91223 Bretigny sur Orge Cedex, France)

This study examined how localization, via binaural cues, can help the tracking of a sound sequence, interleaved with another sound sequence, across time. The ability to track a sequence was investigated using a temporal irregularity detection task. The temporal irregularity was inserted into a regular isotone sequence (target sequence) interleaved with an irregular sequence of same frequency (masker sequence). Performances were at the chance level when there was no interaural difference between the sequences. In experiment 1 , large improvements of performances were found using several interaural level (ILD) or time (ITD) differences between the two sequences. Manipulating the masker sequence ILD and maintaining the signal-to-noise ratio constant in the best ear (experiment 2 ) or using a monaural presentation of the sequences (experiment 3), data showed that the effect of ILD could not be explained by a monaural processing (best or bad ear listening). Finally, experiment 4 revealed that the effect of binaural cues on tracking a sound sequence was mainly produced by the perceived localisation differences induced by the interaural differences rather than by the physical differences. These results suggested that binaural cues may influence sound sequence tracking via a spatial attention mechanism.

2aPP9. Comparison of pre- and post-cueing in a multiple-source sound localization task. Brian D. Simpson, Douglas S. Brungart (Air Force Res. Lab., 2610 Seventh St., Bldg. 441, Wright-Patterson AFB, OH 45433, brian.simpson@wpafb.af.mil), Robert H. Gilkey (Wright State Univ., Dayton, OH 45435), Nandini Iyer (Air Force Res. Lab., Wright-Patterson AFB, OH 45433), and James T. Hamil (General Dynam., AIS, Dayton, OH 45433)

Although most sound localization research has examined the ability of listeners to determine the location of a single sound presented in a quiet (typically anechoic) environment, most real-world listening situations are more complex, with multiple simultaneous sounds. Here, an initial experiment, designed to examine localization in multisource environments, is described. Listeners judged the location of a target signal (speech or environmental sound, presented normally or time-reversed) masked by up to four simultaneous sounds. In each block of trials, the observation interval was either preceded by, or followed by, a cueing interval, during which the stimulus to be localized was identified. It was expected that these two approaches would lead to different answers, as the associated tasks presumably address different listening strategies (i.e., analytic listening versus monitoring). The results indicate that, in all conditions, localization errors increase as the number of simultaneous sources increases. Moreover, performance degrades more rapidly in the post-cue condition than in the pre-cue condition. Surprisingly, this difference occurs for as few as two simultaneous sources, suggesting that there is a substantial cost when listeners are asked to remember what sounds were present and where those sounds were located in a complex auditory environment. [Work supported by AFOSR.]

2aPP10. Rate limitation and onset dominance in the discrimination of high-frequency click trains with dynamic envelope ITDs. G. Christopher Stecker (Dept. of Speech \& Hearing Sci., Univ. of Washington, 1417 NE 42nd St., Seattle, WA 98105, cstecker@u.washington.edu)

Several studies have shown that binaural processing at high carrier frequency is limited to relatively slow modulations, suggesting profound consequences for binaural processing of high-rate electrical stimulation by cochlear implants (CIs). In this study, we measured normal-hearingenvelope ITD thresholds for Gaussian impulses (clicks) with $4000 \mathrm{~Hz}$ carrier frequency and $2 \mathrm{~ms}$ total duration (BW approximately $900 \mathrm{~Hz}$ ) and for 16-click trains with rates of 100,200 , or 500 pps. For click trains, right-leading ITDs were static (condition RR), increased linearly from 
diotic (condition 0R), or decreased linearly toward diotic (condition R0). Thresholds were calculated from the largest ITD in each stimulus (i.e., the onset of R0 or offset of $\mathrm{OR}$ trains). Consistent with previous results for normal hearing and bilaterally implanted subjects, thresholds were strongly dependent on ICI. Low-rate (100 pps) thresholds improved for 16-click trains relative to single clicks in all conditions, while high-rate (500 pps) thresholds were similar to single clicks for RR and R0 stimuli. In condition $0 \mathrm{R}, 500 \mathrm{pps}$ ITD thresholds were elevated and comparable to monaural rate discrimination. The results suggest an inability to lateralize high-rate stimuli in the absence of onset ITDs and have important consequences for binaural information processing with cochlear implants.

2aPP11. Evidence for active suppression of masker locations in spatially separated multitalker conditions. Kachina Allen, Simon Carlile, David Alais (Dept. of Physiol., Univ. of Sydney, F13 Univ. of Sydney 2006, NSW Australia, kachina_allen@yahoo.com), and Karen Froud (Columbia Univ., New York, NY 10027)

Three experiments tested active suppression of masker locations. Using a nonword corpus, spatial release from masking (SRfM) of $3.1 \mathrm{~dB}$ was calculated using masked thresholds $(67 \%)$ of colocated and separated (20 deg) masker and target. Mirrored conditions were completed, with results pooled between hemispheres. In experiment $1,80 \%$ of trials were masked 20 deg left of the central target. Random (10\%) trials played targets at the expected masker location, and (10\%) at the symmetrically opposite side, with maskers $20 \mathrm{deg}$ laterally displaced. Thresholds revealed 2.6-dB reduction at the masker location compared to symmetrically opposite (null) locations. Experiment 2 used a central target with masker played 20 deg to the left in $80 \%$ of trials. Random (20\%) trials (unexpected) played the masker at the symmetrically opposite side. Target thresholds were reduced by $2.1 \mathrm{~dB}$ when masker location was unexpected. Both experiments indicate active suppression of expected masker locations, suggesting masker suppression may contribute to SRfM. Experiment 3 played $80 \%$ of trials with maskers 20 deg left of the central target. Random (10\%) trials played the target $40 \mathrm{deg}$ to the left of center, and (10\%) at the symmetrically opposite side, with the masker 20 deg laterally displaced. Reduced target thresholds $(2.0 \mathrm{~dB})$ indicated that masker suppression also extends laterally, beyond the expected masker location.

2aPP12. Lateralization of amplitude-modulated bone-conducted ultrasounds: Discrimination of interaural time and intensity differences. Takuya Hotehama and Seiji Nakagawa (Natl. Inst. of Adv. Industrial Sci. and Technol. (AIST), 1-8-31 Midorigaoka, Ikeda, Osaka 563-8577, Japan, t-hotehama@ aist.go.jp)

Ultrasonic vibration generates a sensation of sound via bone conduction; the phenomenon is called bone-conducted ultrasonic (BCU) hearing. Complex sounds also can be perceived by an amplitude-modulated BCU (AM-BCU). In this study, to investigate whether listeners can use an interaural time difference (ITD) in the amplitude envelope and an intensity difference (IID) as cues for lateralization, difference limens for an ITD (ITDL) and an IID (IIDL) for AM-BCU were evaluated. Thirty-kHz sine waves modulated by low-passed noises with cutoff frequencies of 250 , 500, 1000, 2000, and $4000 \mathrm{~Hz}$ were used as the AM-BCU stimuli. ITDLs and IIDLs for air-conducted (AC) low-passed noises which correspond to modulator of the AM-BCU were also evaluated. Both ITDLs and IIDLs for AM-BCU were observed; AM-BCU was larger in ITDL and smaller in IIDL than AC. The results suggest that it is possible to lateralize AM-BCU using ITD in the envelope and/or IID; however, IID is a more effective cue than ITD.
2aPP13. Modulation masking in monaural and binaural conditions. Monika Kordus (Inst. of Acoust., Adam Mickiewicz Univ., Umultowska 85, 61-614 Poznan, Poland)

The shape of hypothetical modulation filters was measured in monaural and binaural signal presentation. A sinusoidal 4-kHz carrier was amplitude modulated by low-low-noise (LNN) [A. Kohlrausch et al., Acta Acust. 83, 659-669 (1997)] and a probe tone of a frequency varying from 2 to $256 \mathrm{~Hz}$. Using a three-interval forced-choice (3IFC) procedure the threshold of modulation by the probe tone was determined for the LNN of $16-\mathrm{Hz}$ center frequency and $8-\mathrm{Hz}$ bandwidth, and $64-\mathrm{Hz}$ center frequency and 8-, 16-, or 32-Hz bandwidth. In the monaural condition, signals were presented to the right ear. In the binaural condition, modulation by the LNN was presented to the right ear, and modulation by the probe tone to the left ear. Results showed that masking in the modulation rate domain is characterized by a broad tuning with the highest values of about $17 \mathrm{~dB}$ for the monaural condition, and 4 to $9 \mathrm{~dB}$ in the binaural condition occurring for probe-tone frequency equal the center frequency of the LNN. The large effect in binaural condition confirms the idea that modulation detection by hypothetical modulation filter bank occurs on a higher level in the auditory system. [Work supported by the Polish Ministry of Education and Science, Grant No. 4T07B03230.]

2aPP14. Frequency dependency of illusory continuity in binaural hearing with respect to interaural time difference. Mamoru Iwaki and Hiroki Kitajima (Grad. School of Sci. and Technol., Niigata Univ., 8050 Ikarashi-2, Niigata, 950-2181, Japan, iwaki@bc.niigata-u.ac.jp)

Illusory continuity is a psychoacoustical phenomenon: when the absence portion of a tone is padded with sufficiently powerful white noise, an intermittent pure tone is perceived as though it were continuous. Many studies have examined this psychoacoustical illusion in monaural hearing, which supports that illusory continuity is observed when no clear evidence of discontinuity exists and when the power of the white noise is sufficiently large to mask the pure tone. In binaural hearing, the authors reported that illusory continuity was observed and that it was related with interaural time difference (ITD) for a single target pure tone of $500 \mathrm{~Hz}$ [M. Iwaki and N. Nakamura, INTERSPEECH/EUROSPEECH-2003 (2003) pp. 2065-2068]. This study investigated illusory continuity in binaural hearing for wider frequency ranges of $250-2000 \mathrm{~Hz}$. Results showed that in the lower frequency range, the threshold level of illusory continuity in binaural hearing depended on ITDs between the tone target and noise masker. The increase of the threshold level was minimal when the target and masker have the same ITD. In the higher frequency range, the illusory continuity was independent of differences of ITDs.

2aPP15. Localizing noise in rooms via steady-state interaural time differences. Brad Rakerd (Dept. of Communicative Sci. and Disord., Michigan State Univ., E. Lansing, MI 48824, rakerd@msu.edu), William M. Hartmann, and Eric Pepin (Michigan State Univ., E. Lansing, MI 48824)

Noise lateralization experiments with headphones show that the steady-state interaural time difference (ITD) is, or is not, a valuable cue for sound lateralization depending on the height of the peak of the interaural cross-correlation function (cc). To discover whether these headphone results could be extended to the localization of sound in rooms, binaural recordings were made of one-third-octave noises in diverse environments: a dry classroom, a laboratory, and a reverberation room. Sound sources and a KEMAR recording manikin were moved about in each room to vary the $\mathrm{cc}$ from 0.2 to 0.8 . The recordings, with ITDs digitally controlled, were presented to five listeners by headphones to measure listener sensitivity to ITD variations. It was found that sensitivity was almost entirely a function of $\mathrm{cc}$ with little weight attached to the actual room environment. ITD thresholds decreased monotonically with increasing cc. In agreement with earlier headphone studies using computed noises instead of room record- 
ings, typical thresholds were higher by a factor of about 6 for a $2800-\mathrm{Hz}$ band where envelope ITD is used compared to a $700-\mathrm{Hz}$ band where waveform ITD is used. [Work supported by the NIDCD.]

2aPP16. Investigating the buildup of precedence effect using reflection masking. Jess Hartcher-O'Brien, M. Buchholz (Macarthur Auditory Res. Ctr. Sydney (MARCS), Bldg. 5, Bankstown Campus, UWS, Locked Bag, 1797, Penrith South DC), and M. Buchholz (Tech. Univ. of Denmark, DK-2800 Kgs. Lyngby, Denmark)

The auditory processing level involved in the build-up of precedence [Freyman et al., J. Acoust. Soc. Am. 90, 874-884 (1991)] has been investigated here by employing reflection masked threshold (RMT) techniques. Given that RMT techniques are generally assumed to address lower levels of the auditory signal processing, such an approach represents a bottom-up approach to the buildup of precedence. Three conditioner configurations measuring a possible buildup of reflection suppression were compared to the baseline RMT for four reflection delays ranging from 2.5-15 ms. No buildup of reflection suppression was observed for any of the conditioner configurations. Buildup of template (decrease in RMT for two of the conditioners), on the other hand, was found to be delay dependent. For five of six listeners, with reflection delay $=2.5$ and $15 \mathrm{~ms}$, RMT decreased relative to the baseline. For 5- and 10-ms delay, no change in threshold was observed. It is concluded that the low-level auditory processing involved in RMT is not sufficient to realize a buildup of reflection suppression. This confirms suggestions that higher level processing is involved in PE buildup. The observed enhancement of reflection detection (RMT) may contribute to active suppression at higher processing levels.

2aPP17. Temporal processes involved in simultaneous reflection masking. Joerg M. Buchholz (CAHR, Acoust. Technol., Tech. Univ. of Denmark, Oersted Plads, Bldg. 352, DK-2800 Kgs. Lyngby, Denmark)

Reflection masking refers to the specific masking condition where a test reflection is masked by the direct sound. Employing reflection masking techniques, Buchholz [J. Acoust. Soc. Am. 117, 2484 (2005)] provided evidence that the binaural system suppresses the test reflection for very short reflection delays and enhances the test reflection for large delays. Employing a 200-ms-long broadband noise burst as input signal, the critical delay separating these two binaural phenomena was found to be 7-10 $\mathrm{ms}$. It was suggested that the critical delay refers to a temporal window that is employed by the auditory system to analyze the considered stimulus. In the present study, the dependency of this critical delay on the duration of the input signal (i.e., broadband noise) has been investigated. The psychoacoustical results revealed that the critical delay decreases with decreasing stimulus duration, resulting in a critical delay of about $2-3 \mathrm{~ms}$ for 20-ms-long stimuli. Hence, for very short stimuli the temporal window or critical delay exhibits values similar to the auditory temporal resolution as, for instance, observed in gap-detection tasks. It is suggested that the larger critical delay observed for longer stimuli is due to the auditory system focusing on lower signal frequencies.

2aPP18. Psychophysical estimates of cochlear tuning using simultaneous and nonsimultaneous masking. Masashi Unoki and Ryota Miyauchi (School of Information and Sci., JAIST, 1-1 Asahidai, Nomi, Ishikawa, 923-1292 Japan, unoki@ jaist.ac.jp)

This study attempts to estimate the tuning of an auditory filter, through both simultaneous and forward masking experiments with a notched-noise masker or a sinusoidal masker, to investigate how the effects of suppression affect estimates of frequency selectivity. In these experiments, masked thresholds of the short probe signal (5-ms ramps, no steady state) centered in the masker or delayed from the masker were measured for normal-hearing subjects as a function of the signal frequency $(0.5,1.0$, 2.0 , and $4.0 \mathrm{kHz})$, signal level $(10,20$, and $30 \mathrm{~dB}$ SL), signal delay, and notch-width conditions in the notched-noise masker, or the masker fre- quency in the sinusoidal masker. ERBs were calculated from derived auditory filters to compare the cochlear tuning. The estimated tunings became much sharper as the center frequency was increased. Moreover, the difference between the tunings of the filter at lower frequencies with either form of masking tended to be smaller than that at higher frequencies. The tuning may be affected by residual effects of suppression due to lower masker components below the signal frequency. [Work supported by special coordination funds for promoting science and technology of young researchers from the Japanese Ministry of Education.]

2aPP19. Practice effects in comodulation masking release. Cynthia M. Zettler and Marsha G. Clarkson (Dept. of Psych., Georgia State Univ., P.O. Box 5010, Atlanta, GA 30302, czettler@student.gsu.edu)

Substantial research has explored the acoustic factors influencing comodulation masking release (CMR), but the impact of practice has not been addressed. The current study evaluated the effect of experience on CMR thresholds. Ninety-eight adults listened to CMR stimuli through headphones once a week for 3 weeks. CMR was measured as the difference in threshold between a reference condition with one 20-Hz-wide amplitude-modulated noise band centered on a $1000-\mathrm{Hz}$, pure-tone signal, and a modulated masker condition having eight additional noise bands comodulated with the on-signal band. Thresholds were estimated in a single-interval, maximum-likelihood procedure. Hierarchical linear modeling tested the effect of practice on thresholds and CMR. Analyses indicated that thresholds improved significantly with practice $(p<0.001)$ for both masking conditions. On average, reference thresholds improved 5.41 $\mathrm{dB}$ per visit $(\mathrm{SE}=0.703)$, and modulated masker thresholds improved $3.15 \mathrm{~dB}$ per visit $(\mathrm{SE}=0.495)$. Total $\mathrm{CMR}$ decreased significantly $(p$ $<0.01)$ by $1.98 \mathrm{~dB}$ per visit $(\mathrm{SE}=0.701)$, most likely because performance in the reference condition improved more than performance in the modulated masker condition. Results indicate that practice improves performance in CMR masking conditions and that total CMR decreases with experience.

2aPP20. Perceptual judgment in synchronization of two complex tones and its relation to the cochlear delays. Eriko Aiba (Grad. School of Music, Kyoto-City Univ. of Arts, 13-6 Oe-kutsukake-cho, Nishikyo-ku, Kyoto, 610-1197 Japan, m06901@kcua.ac.jp) and Minoru Tsuzaki (Kyoto-City Univ. of Arts, Japan)

Onset synchrony of components is widely assumed to be an important cue for perceptual unification as a single tone. However, even if the components begin simultaneously, their temporal relation might not be preserved at the cochlear level. The cochlear delay shifts the arrival of a lower component slightly but systematically behind a higher component. This experiment uses two complex tones as a stimulus to investigate whether the cochlear delay imposes a systematic bias in judging the synchrony of two complex tones. The results are compared with those of our previous experiment using two sinusoidal signals, which indicated that the accuracy of synchrony judgment was worse when the higher component preceded the lower one than in the opposite case. These results will be compared with those of a pioneering study, which indicated that the region of judgment on synchrony of complex tones was greater than $30 \mathrm{~ms}$. [Work supported by the Grant-in-Aid for Scientific Research A, No.16200016, Japan Society for the Promotion of Science.]

2aPP21. Sequential amplitude-modulation interference across auditory channels and across ears. Magdalena Wojtczak, Melanie J. Gregan, and Neal F. Viemeister (Dept. of Psych., Univ. of Minnesota, 75 East River Rd., Minneapolis, MN 55455)

Forward masking has been demonstrated in the amplitude-modulation (AM) domain [M. Wojtczak and N. F. Viemeister, J. Acoust. Soc. Am. 118, 3198 (2005)] for masker and signal imposed on the same carrier. This study explores an effect of AM on detection of AM imposed sequentially 
on a different carrier. Data show that a $40-\mathrm{Hz}$ AM (interferer) imposed on a $2-\mathrm{kHz}$ carrier affects detection of a $40-\mathrm{Hz}$ AM (signal) imposed on a $5.5-\mathrm{kHz}$ carrier after the offset of the interferer AM. The effect is analogous (and similar in size for the shortest interferer-signal delays) to modulation detection interference for simultaneous interferer AM. In another condition, two independent noise samples were presented to opposite ears. In one ear, a $40-\mathrm{Hz}$ masker AM was imposed on the carrier. In the opposite ear, a 40- Hz signal was imposed on the carrier after the offset of the masker modulation. A forward-masking effect similar to but smaller in size than that in monaural presentation was observed. The data from both experiments suggest that the forward-masking effect in the modulation domain occurs at central stages of processing, where information is combined across peripheral channels and where binaural interactions occur. [Work supported by NIH Grants DC00683 and DC006804.]

2aPP22. The influence of broadened auditory tuning on acrossfrequency integration of speech. Eric W. Healy and Kimberly A. Carson (Dept. of Commun. Sci. and Disord., Arnold School of Public Health, Univ. of South Carolina, Columbia, SC 29208)

Previous work has suggested that hearing-impaired (HI) listeners may have a deficit in their ability to integrate speech information across different frequencies. Performance of HI listeners was poorer than that of normal-hearing $(\mathrm{NH})$ listeners on across-frequency tasks, and performance dropped more steeply when the signal was degraded through acrossfrequency asynchrony [E.W. Healy and S.P. Bacon, J. Speech Lang. Hear. Res. 45, 1262-1275 (2002)]. In the current study, the role of broadened auditory tuning in this deficit was investigated. Broad tuning was simulated in NH listeners using noise-vocoded sentences and manipulating the slopes of the amplitude-modulated carriers. It was found that intelligibility of synchronous carriers was poorest in the broadest slope conditions, suggesting that poor integration of synchronous cues may be at least partially attributable to broad tuning. Performance reductions as a function of asynchrony were also found to be greatest in the broadest conditions, but only when analysis bands matched presentation bands and not when analysis bands were held constant. It is concluded that broadened auditory tuning may account for at least a portion of the across-frequency deficit observed in HI listeners. [Work supported by NIH.]

2aPP23. Effect of flanker band number and excitation pattern symmetry on envelope comparisons in masking release. Ifat Yasin (Dept. of Exp. Psych., South Parks Rd., Univ. of Oxford, Oxford OX1 3UD, UK), Deborah A. Fantini (Univ. of Essex, Colchester CO4 3SQ, UK), and Christopher J. Plack (Lancaster Univ., Lancaster LA1 4YF, UK)

Greater masking release (MR) is observed when narrow-band noise flanker bands (FBs) are lower, rather than higher, than the signal frequency. This may be due to more effective signal coding on the highfrequency side of the excitation pattern (EP) [B. C. J. Moore and U. Jorasz, J. Acoust. Soc. Am. 100, 2373-2379 (1996)]. If so, masking of a high-frequency signal (asymmetric EP) should lead to a greater MR between FBs below and above the signal frequency than for a lowerfrequency signal (symmetrical EP). Masked signal thresholds were compared for pure-tone signals $(0.5,1$, or $2 \mathrm{kHz})$ masked by a narrow-band noise masker centered at the signal frequency [on-frequency band (OFB)] and two (three) FBs at 2-ERB intervals, below (above) the signal frequency. Masked signal thresholds were also measured in the absence of FBs. The OFBs and FBs were modulated at a modulation depth, $m$, of 0.7. FB envelopes were the same as each other and that of the OFB (comodulated, C), the same as each other but independent of OFB (incoherent, I), or not modulated (unmodulated, U). No clear relationship was found between signal EP symmetry and MR magnitude. MR is susceptible to the computation used, i.e., $[(\mathrm{I}+\mathrm{U})-\mathrm{C}]$ or $[\mathrm{I}-\mathrm{C}]$ as well as level cues.
2aPP24. Timing perception of tonal sequences connected with glides. Satomi Tanaka (Grad. School of Music, Kyoto City Univ. of Arts, 13-6 Kutsukake-chou, Oe, Nishikyo-ku, Kyoto-city, Kyoto 610-1197 Japan, m06902@kcua.ac.jp), Minoru Tsuzaki (Kyoto City Univ. of Arts), and Hiroaki Kato (ATR Cognit. Information Sci. Labs./NICT)

When we hear continuous sound signals, we extract the timing between each segment although each segmental boundary is acoustically unclear. In order to investigate on which points we perceive the temporal structures in sound signals, perceptual experiments were conducted to measure the sensitivity in detecting the temporal deviation by using sound signals containing frequency glides. The following question was raised: What would provide a more effective cue to detect a change in the temporal structures, the starting point or the ending point of glides? Five segments of steady parts, of which high and low were alternated, were connected with four frequency glides, while the amplitude was fixed. By randomizing the duration of glide parts and adjusting the duration of steady parts compemsatively, one can exclusively add a certain degree of fluctuation on the starting point or ending point of glides. The task was to detect a temporal deviation from an isochronous structure. The thresholds of detection were measured within three ranges of the center frequency. The detectability tended to be better under the starting point condition than under the ending point condition. The results were discussed in relation to a computational model of event detection. [Work supported by JSPS.]

2aPP25. Timing perception of sequences with no obvious onset: Isochrony detection in sequences of complex tones connected by formant glides. Minoru Tsuzaki (Kyoto City Univ. of Arts, NICT/ATR Spoken Lang. Commun. Labs., KCUA, 13-6 Kutsukake-cho, Oe, Nishikyo-ku, Kyoto 610-1197, Japan), Satomi Tanaka (Kyoto City Univ. of Arts, Japan), and Hiroaki Kato (ATR Cognit. Information Sci. Labs./ NICT)

To investigate the perceptual cues that detect arrivals of new auditory events, the detectability of deviation applied to an isochronous temporal structure was measured. The stimuli were sequences of complex tones whose formant frequencies alternate between two steady values. Five steady parts were connected by four formant glides. The isochronous structure was introduced exclusively in the intervals between the starting points of the glides or in the intervals between the ending points by adjusting the durations of the glides and the steady parts in a compensatory manner. Since the harmonic components stop and start one after the other during the glides, it is difficult to define the arrival of new events in an entirely physical view. The perceptual difference in detectability between the starting and ending conditions and its dependency on the frequency region could reveal the perceptual cues that are effective in extracting temporal relations of auditory events. The results will be compared with those obtained with sinusoidal stimuli whose frequencies were modulated in a similar way as the formant frequency in this paper, and they will be discussed by reference to a computational model of auditory event detection. [Work supported by JSPS No. 16200016.]

2aPP26. Human performance in detecting audio-visual asynchrony. Armin Kohlrausch, Steven van de Par (Philips Res. Labs. Eindhoven (WO02), HTC 36, NL-5656AE Eindhoven, The Netherlands, armin.kohlrausch@philips.com), Rob van Eijk (Techn. Univ. Eindhoven, NL-5600 MB Eindhoven, The Netherlands), and James F. Juola (Univ. of Kansas, Lawrence, KS 66045)

The perception of audio-visual delays by human observers is typically characterized by two quantities: the point of subjective equality (PSE) and the sensitivity to asynchrony. The PSE can be derived from a temporal order judgment (TOJ) procedure or from the outcomes of a synchronoussuccessive response paradigm. Sensitivity to asynchrony is derived from the steepness of the response curve in the TOJ paradigm. In this contribution we present data that show that PSE estimates derived from TOJ measurements are much more variable across observers than those based on synchronous-successive data. Two synchronous-successive methods were 
used with different response categories: (1) two categories: asynchronous, synchronous, and (2) three categories: audio first, synchronous, video first. Both synchronous-successive methods yielded similar results. A slight influence of stimulus type on PSE estimates derived from synchronoussuccessive data was observed. To conclude, we analyzed discriminability values obtained with a two-alternative forced-choice procedure. Discriminability is better near the edge than in the middle of the synchronous response category. This suggests that categorical perception might play a role in audio-visual synchrony perception.

2aPP27. The effect of marker duration on time shrinking. Yoko Fujishima and Yoshitaka Nakajima (Faculty of Inst. of Design, Kyushu Univ. 4-9-1 Shiobaru, Minami-ku, Fukuoka, 815-8540, Japan, fujishim@gsd.design.kyushu-u.ac.jp)

Time shrinking is an illusion of time perception. This illusion takes place in stimulus patterns of three successive sound bursts (markers) of equal duration marking two empty time intervals, the preceding time interval $(\mathrm{P})$ and the standard time interval $(\mathrm{S})$. When $\mathrm{S}$ is longer than $\mathrm{P}, \mathrm{S}$ is often underestimated. In this study, we examined whether the duration of the bursts (in this case, pure tones) could affect this illusion. Time shrinking always took place when $\mathrm{S}-\mathrm{P}$ was less than or equal to $60 \mathrm{~ms}$, and the marker duration did not affect the amount of underestimation of $\mathrm{S}$ in this range. However, the illusion took place only for the shortest marker duration of $20 \mathrm{~ms}$ when $\mathrm{S}-\mathrm{P}=80 \mathrm{~ms}$. The results roughly agreed with previous data. [Work supported by JSPS and 21st Century COE, Kyushu University.]

2aPP28. The role of spectral factors in the occurrence of the gap transfer illusion. Tsuyoshi Kuroda, Yoshitaka Nakajima (Faculty of Design, Dept. of Acoust. Design, Kyushu Univ., 4-9-1 Shiobaru, Minami-ku, Fukuoka 815-8540, Japan, kuroda@gsd.design.kyushu-u.ac.jp), Shimpei Tsunashima, Tatsuro Yasutake, and Shiro Mitsuba (Kyushu Inst. of Design, Minami-ku, Fukuoka 815-8540, Japan)

When a long ascending frequency glide with a gap at the temporal middle and a short continuous descending glide cross at their central position, observers report that the gap belongs to the short glide in spite of the physical position of the gap. This phenomenon is called the gap transfer illusion. We employed multiple-component crossing patterns and systematically varied the spectral slopes or formants of glides. In patterns that contained glides consisting of three harmonic components, the gap transfer illusion occurred when the crossing glides had equal spectral slopes, while the illusion was inhibited when the crossing glides had different spectral slopes. In patterns that contained 25 harmonic components representing formants of Japanese vowels, the gap transfer illusion took place only when the crossing glides had the same formants. Although Nakajima et al.
(2000) explained the gap transfer illusion in terms of the proximity of onsets and terminations, the present results show that it is also important to consider the role of spectral factors. [Work supported by JSPS and 21st Century COE, Kyushu University.]

2aPP29. An upper bound on the temporal resolution of human hearing. Milind N. Kunchur (Univ. of South Carolina, Dept. of Phys. \& Astron., Columbia, SC 29208, kunchur@sc.edu)

This work obtained an upperbound of about $5 \mathrm{~ms}$ for the temporal resolution of hearing by studying human discriminability for bandwidth restriction by low-pass filtering. While low-pass filtering affects both the spectrum and temporal definition of the signal, the spectral changes in this experiment fall below the known just noticeable differences, so that the threshold seems to reflect the neural temporal-discrimination of the auditory system, rather than its ability to distinguish spectral cues based on tonotopic excitation patterns. The present result shows that human temporal resolution extends down to time scales much shorter than found in the past. Rough estimates of the expected resolution, based on convergencies in the cochlear nucleus and subsequent stages, yields values in a range that is consistent with the present experimental result.

2aPP30. Models of musical timbre using cortical spectro-temporal receptive fields and temporal codes. Mounya Elhilali (Inst. for Systems Res., Dept. of Elec. and Comput. Eng., A.V. Williams Bldg., Univ. of Maryland, College Park, MD 20742, mounya@isr.umd.edu), Daniel Pressnitzer (CNRS Université René Descartes Paris, 75005 Paris, France), and Shihab Shamma (Univ. of Maryland, College Park, MD 20742)

It has been customary to propose several "descriptive" definitions to quantify timbre as one of the basic attributes of sound. Multidimensional scaling techniques have proposed several acoustic dimensions to characterize the timbre of subsets of sounds. Yet, in the general case, timbre is an intricate percept difficult to relate to concise acoustic cues. Timbre probably reflects the dynamic interactions between spectral and temporal characteristics of sound that play a crucial role in identifying natural sources and convey a wealth of information about the environment. Recent physiological investigations revealed possible neural mechanisms to extract and represent the spectral and temporal information in complex sounds. At the level of the primary auditory cortex, spectro-temporal receptive fields exhibit a multiscale representation that captures many aspects of dynamic sound spectra. In the current study, we explore the potential correspondence between these cortical properties and the identification of musical instruments. We investigate the use of such representation as a quantitative descriptor of musical timbre, as validated by behavioral data from human subjects. Additionally, we examine biologically plausible coding strategies in this multidimensional cortical mapping involving spike-timing information. 


\title{
Session 2aSC
}

\section{Speech Communication: Prosody (Poster Session)}

\author{
Victoria B. Anderson, Cochair \\ Univ. of Hawaii, Dept. of Linguistics, 1890 East West Rd., Honolulu, HI 96822 \\ Keikichi Hirose, Cochair \\ Univ. of Tokyo, Dept. Information and Communication Engineering, 7-3-1 Hongo, Bunkyo-ku, Tokyo 113-0033, Japan
}

\section{Contributed Papers}

\begin{abstract}
All posters will be on display from 8:00 a.m. to 12:00 noon. To allow contributors an opportunity to see other posters, contributors of odd-numbered papers will be at their posters from 8:00 a.m. to 10:00 a.m. and contributors of even-numbered papers will be at their posters from 10:00 a.m. to 12:00 noon.
\end{abstract}

2aSC1. The effects of speaking rate and tonal contexts on target undershoot of mandarin tone 3. Jia-Shiou Liao (Dept. of Linguist., Univ. of Texas, Calhoun Hall 501, 1 Univ. Station B5100, Austin, TX 78712-0198)

An investigation of the effects of speaking rate and tonal contexts on target undershoot of Mandarin tone 3 (low or low-falling-rising) was conducted. Data were from seven male and eight female subjects, all native speakers of Mandarin. The test phrase comprises three $/ \mathrm{ma} / \mathrm{s}$ in a row, which were put in a sentence to provide a natural context. The second $/ \mathrm{ma} /$ was always tone 3 , preceded and followed by tone 1 (high-level), tone 2 (mid-rising), tone 3 or tone 4 (high-falling), resulting in 16 distinct combinations. Subjects read sentences at two relatively different rates: slow and fast. The fundamental frequency (F0) and duration of each $/ \mathrm{ma} /$ were measured at the onset and offset of its vowel. In addition, the duration of the second /ma/ and F0 at its midpoint were measured. Quantitative analysis of the rate of change in F0 suggests, by and large, that the F0 control systems are not always the same in slow and fast speech within the same tonal contexts. Furthermore, undershoot phenomena occur more often statistically across a syllable boundary than within a syllable boundary. Moreover, the F0 control system produces rates with less random fluctuation across the syllable boundary than within the syllable boundary.

2aSC2. Analysis on the effects of tonal coarticulation at word and nonword syllable boundaries of Mandarin based on the tone nucleus model. Qinghua Sun, Hirose Keikichi, and Minematsu Nobuaki (Univ. of Tokyo, 7-3-1 Hongo, Bunkyo-ku, Tokyo 113-8656 Japan, qinghua@gavo.t.u-tokyo.ac.jp)

As a typical tonal language, Mandarin has four lexical tone types, each of which has its own intrinsic pattern when uttered in isolation. However, when produced in context, the tonal contours present many variations, even if we specifically address the tone nucleus, which is defined as a stable part in a syllable. This study describes how and to what extent the word boundary affects tonal coarticulation in continuous speech. Although several previous works have investigated how the prosodic features of syllables are affected by their surrounding syllable tone types, most of them selected nonsense syllables uttered in a carrier sentence as study targets, which is far from natural situation. In this paper, after preparing a Mandarin speech corpus, in which all tone-type combinations of disyllables are included with both intraword and interword cases, the degree of concatenation is compared for intraword and interword cases, viewing their tone component's F0 means and ranges of tone nuclei. All target di-syllables are uttered in a phrase. Results indicate that the degree in the intraword and interword cases differ greatly depends on the tone combination. The results were applied into our tone component prediction system, which is based on the tone nucleus model.

2aSC3. The command-response model for F0 contours and its application to phonetics and phonology of tone. Hiroya Fujisaki (Univ. of Tokyo, Tokyo, Japan)

The command-response model for the F0 contours, first proposed for nontone languages such an Japanese and English, has been extended by the author and his co-workers to cover F0 contour of tone languages and has been proved to be applicable to several dialects of Chinese including Mandarin, Cantonese, and Shanghainese, as well as other tone languages including Thai and Vietnamese. Namely, it allows one to approximate F0 contours of these languages/dialects with a very high accuracy using positive and negative tone commands. This paper first presents some results of analysis of F0 contours of these languages/dialects to demonstrate that the model can capture the acoustic-phonetic characteristics of their tones quite well. It then shows that coarse qualitative distinctions in the pattern of tone commands serve to distinguish tones within a language/dialect, as well as to distinguish tone systems of different languages/dialects. Thus the model proves to be a useful tool, not only in the studies of phonetic features of tones, but also in the studies of phonological structure of tone systems.

2aSC4. Tone sandhi effect on Chinese speech perception. Li Hsieh and Ya-Ju Yu (Dept. of Commun. Sci. and Disord., Wayne State Univ., 207 Rackham Hall, 60 Farnsworth, Detroit, MI 48202)

Mandarin Chinese has four tones with various sandhi processes. The third tone sandhi process is when a third tone (T3) becomes a second tone (T2) before another third tone (T3). Previous studies on third tone sandhi used connected speech in their experiments. This study examines the third tone sandhi effect on Chinese listeners in a nonconnected speech condition. Full-tone Chinese syllables were recorded in isolation. Ten Chinese listeners participated in tonal discrimination tasks which consisted of combinations of T2 and T3 (i.e., T22, T23, T32, T33). Three interstimulus intervals (ISIs) (i.e., 50, 250, $500 \mathrm{~ms}$ ) and three types of pair conditions (minimal-, rhyme-, mixed-pairs) were introduced in the experiments. The findings of this study confirm the phonological hypothesis that the third tone sandhi effect has a great impact across all the pair conditions. When discriminating T23, Chinese listeners had longer response times and more errors than other tone pairs. The phonological phenomenon of third tone 
sandhi persists regardless of connected or nonconnected speech conditions in Chinese spoken language. Differences in processing the tone sandhi pairs (T23) and the nontone sandhi pairs (T32) reveal the psychological reality of the third tone sandhi phonological rule.

2aSC5. A cross-language comparison of the use of stress in word segmentation. Michael D. Tyler (MARCS Auditory Labs., Univ. of Western Sydney, Bldg. 5, Bankstown Campus, Locked Bag 1797, Penrith South DC NSW 1797, Australia), Pierre Perruchet (Université de Bourgogne, Esplanade Erasme, 21000, Dijon, France), and Anne Cutler (Max Planck Inst. for Psycholinguist., 6500 AH Nijmegen, The Netherlands)

In spite of our illusions to the contrary, there are few acoustic cues to word boundaries in spoken language. While statistical probabilities between adjacent speech units provide language-general information for speech segmentation, this study shows that language-specific information may also play an important role. An $f 0$ excursion, a correlate of stress, was provided on the first or last syllable of each word in an artificial language. As expected, French listeners succeeded with final-syllable stress but not initial-syllable stress. However, Dutch listeners appeared to benefit from both initial and final stress, rather than initial stress only. When the results were split according to the language background of the talker (French or Dutch), it became clear that the Dutch listeners only benefited from initial stress when the talker was Dutch, but not when the talker was French. Given that the majority of Dutch listeners spoke French, it is suggested that they readily adapted their word segmentation strategies when faced with French-sounding input (i.e., French talker or final stress). In support of this, Australian English listeners with no knowledge of French clearly benefited from initial stress only, consistent with English stress patterning. [Work supported by Conseil Regional de Bourgogne, NWO, CNRS, NIHDC00403.]

2aSC6. Acoustic and spectral correlates of lexical stress. Anthony O. Okobi (Speech and Hearing Bioscience and Technol., Harvard-MIT Div. of Health Sci. and Technol., Cambridge, MA 02139)

Acoustic and spectral parameters that differentiate between primary and nonprimary stressed vowels were determined using two-syllable novel words with identical syllable compositions. The location of the high focal pitch accent within a declarative carrier phrase was varied using an innovative object naming task that allowed for a more natural and spontaneous manipulation of phrase-level accentuation. Results showed that when the high focal pitch accent was on the novel word, vowel differences in pitch prominence, intensity prominence, and vocal tract filter corrected amplitude of the first harmonic $\left(H 1^{*}\right)$ accurately distinguished primary from nonprimary stressed vowels. Vowel differences in first formant frequency, the spectral approximations of open quotient, $H 1^{*}-H 2 *$, and first formant bandwidth, $H 1^{*}-A 1^{*}$, also correlated with lexical stress under this condition. Correlates of lexical stress that were differentiating under all conditions tested were spectral tilt, $H 1^{*}-A 3^{*}$, noise at high frequencies, and duration. Furthermore, results indicated that these lexical stress vowel differences were augmented when the high focal pitch accent was on the novel word. This became apparent after a formula was devised to correct for the masking effect of phrase-level accentuation on the spectral tilt measurement. [Work partially supported by NIH T32-DC00038.]

2aSC7. The acoustic correlates of stress in compounds. Tuuli M. Adams (Linguist. Dept., New York Univ., 719 Broadway, 4th Fl., New York, NY 10003, tuuli.adams@nyu.edu)

The acoustic correlates of linguistic stress are traditionally considered to be intensity, pitch, and segment duration. The current study investigates the correlates of stress in English compounds such as "blackboard" by comparing their interaction with different intonational patterns. This study includes an experiment in which participants pronounced compounds in controlled prosodic and intonational environments and various changes in intensity $(\mathrm{dB})$, pitch $(\mathrm{Hz})$, and duration were recorded for each vowel. Compounds in English are generally said to have a definitive stress pattern that distinguishes them from phrasal sequences; for example, "greenhouse" (for plants) would be stressed only on the first syllable, and its counterpart "green house" (house that is green) has a more even stress pattern. However, until now there has been no empirical study that successfully identifies the acoustic cues that distinguish compounds from phrasal structures. Results of this experiment show that intensity, pitch, and duration participate in a form of cue trading to signify stress in different contexts. This experiment provides new evidence that the acoustic correlates of stress interact with different intonational environments in a consistent way that distinguishes compounds from phrases.

2aSC8. Second occurrence focus and the acoustics of prominence. Jonathan Howell (Dept. of Linguist., Cornell Univ., 203 Morrill Hall, Ithaca, NY 14850,jah238@cornell.edu)

Partee (1991) challenged the significance of the observation that certain adverbs (e.g., only) reliably associate with phonologically prominent words to truth-conditional effect, noting second occurrence (i.e., repeated or given) focus (SOF) appears to lack a phonological realization. Rooth (1996), Bartels (2004), Beaver et al. (2004), Jaeger (2004), and Fry and Ishihara (2005) argued that, while not intonationally prominent, an SOF word can be marked by increased duration and/or increased rms intensity. An acoustic study of verb-noun homophone pairs is reported. Three sophisticated speakers uttered five repetitions of the targets, embedded in discourses, in first occurrence (FOF), SOF, and unfocused (NF) contexts, both pre- and postnuclearly. Syntagmatic comparison shows a durational effect for focus and ordering in the FOF/NF case, yet only an effect of ordering in SOF/NF; similarly, an effect of spectral balance (H1-H3) was found for FOF/NF but none for SOF/NF. Results suggest, contrary to previous assumptions, that simple durational difference is not a straightforward cue of prominence. Sluijter and van Heuven (1996) and Campbell and Beckman (1997) have shown spectral balance calculated by various means is a cue to focus; its absence in these data raises doubts that prominence is reliably produced (let alone perceived) on SOF words.

2aSC9. Intonational distinctions between sentence types in North Kyungsang Korean. Hye-Sook Lee (Cornell Univ., 306 East State St., Apt. 305, Ithaca, NY 14850)

As a lexical pitch accent language, North Kyungsang Korean seems to display a very restricted distribution of intonation. This paper investigates intonational differences between statements and questions in North Kyungsang Korean. In particular, a variety of fo targets are compared between the sentence types, including pitch accent f0 peak, utterance-final edge, pitch range, and f0 valleys. Besides the acoustic analyses, perceptual identification tests are performed to test whether apparently diverging acoustic cues are perceptually significant. Based on ANOVA tests, it turns out that NK Korean questions can be significantly different from statements with respect to the utterance-final edge fo and the relative fo and timing of the rightmost f0 valley. Specifically, questions have higher f0 values at the right edge and higher and earlier occurrences of the rightmost f0 valley. Further, the forced-choice identification tests, whose input materials are manipulated in the similar set of acoustic variables, show that only the utterance-final edge fo is a strong perceptual cue to distinguish between questions and statements. In the meantime, the comparison of yes/no questions and wh-questions demonstrates that there is no common acoustic factor with which the two types of questions are significantly distinguishable. 
2aSC10. The degree of word-initial low tone in Japanese: Syntactic boundaries and speech rate. Hisao Tokizaki (Sapporo Univ., ULS Sumikawa 701, Sumikawa 5-3-8-1, Sapporo, 005-0005 Japan)

This study investigates the nature of word-initial low tone in Japanese. It will be shown that the degree of low tones depends on the speech rate and the number of syntactic boundaries between the word and the immediately preceding word. It will be argued that the phenomena can be explained naturally by boundary mapping from syntax onto phonology (Tokizaki, 1999, 2006). Pierrehumbert and Beckman (1988) and Selkirk and Tateishi (1988) argue that minor phrases are demarcated by initial low tone in unaccented words. Selkirk et al. (2003) argue that degree of low tone depends on whether the word is located at the left edge of a maximal projection of the lexical head (XP). The analysis of spoken data will be presented. Radio news and recited sentences are analyzed by Praat. The pitches of the first and the second mora of a word are measured and compared with the pitch of the high tone in the preceding word. The pitch difference of the first mora and the second is calculated. The result shows that our analysis makes a better prediction for the degree of word-initial low tone than previous analyses. [This project is supported by Grant-inAid for Scientific Research, JSPS, 2006.]

2aSC11. Variability and mismatch: Speakers' cues and listeners' cues. Yuki Hirose (Dept of Lang. Information Sci., The Univ. of Tokyo, 3-8-1 Komaba, Meguro-ku, Tokyo 153-8902, Japan)

The present study questions the natural assumption that the types of cues speakers use are common to those that listeners utilize. The leftversus right-branching ambiguity $(\mathrm{LB} / \mathrm{RB})$ in Japanese complex NPs such as the blue CD-genitive case was the material of interest. In a production study, accompanying visual contexts were manipulated with respect to visual ambiguity: whether there was only one possible target (referent) object corresponding to either RB or LB in each scene, or objects corresponding to both analyses were present in each scene and therefore the ambiguity remains in the visual scene. Phonetic analyses of the collected utterances revealed that when the visual information eliminated the ambiguity, the difference between the LB- and RB-intended utterances was modest and appeared mainly in $F 0$ cues; when the ambiguity remained in the visual context, the prosodic manipulation was made mainly on durational cues. Those utterances were then fed to a perception study. A multiple regression analysis indicated that the cues contributing to $\mathrm{RB} / \mathrm{LB}$ distinctions in listeners mainly showed up on durational cues, suggesting that most types of cues manipulated by speakers who were not aware of the ambiguity did not contribute to listeners' comprehension.

2aSC12. A perception study of syntactic disambiguation using resynthesized speech. Mariko Sugahara (Doshisha Univ., Kyoto, Japan, msugahar@mail.doshisha.ac.jp) and Yuki Hirose (The Univ. of Tokyo, Tokyo, Japan)

It is already well known that when a preceding word (W1) is accented, the following word (W2) is likely to undergo a postaccent downstep. Such a downstep is especially evident in a left-branching structure (henceforth $\mathrm{LB})$ as in [[W1 W2] W3], while it is mitigated in a right-branching structure (henceforth RB) as in [W1 [W2 W3]]. That is, ups and downs of the F0 peak of W2 disambiguate the RB and the LB structure when a postaccent downstep is available (Selkirk and Tateishi, 1991; Kubozono, 1993, Hirose, 2005). Two questions, however, emerge. One is whether or not the magnitude of W3's downstep would ever contribute to the branching disambiguation. The other is what would happen when all words are unaccented, i.e., when no postaccent downstep is available. We carried out a perception study using resynthesized speech as stimuli, to examine those questions. Our results show that neither the $\mathrm{W} 3$ peak values of a postaccent environment nor the W2 peak values of an unaccented environment had significant effect on the LB vs. RB disambiguation. Such results will be compared with Hirose's (2005) production and perception study on spontaneous speech.
2aSC13. The effect of segmental structure on the production of Japanese pitch-accent. Irina A. Shport (Linguist. Dept., Univ. of Oregon, 1290 Univ. of Oregon, Eugene, OR 97403, ishport@uoregon.edu)

Japanese pitch-accent is a fall in fundamental frequency $(F 0)$ after the accented mora. In current models of Japanese pitch-accent contrast, accented and unaccented pitch patterns are predicted to be similar across syllable structure type. This study provides evidence that the segmental make-up of words affects accentual patterns in Tokyo Japanese in terms of their $F 0$ contours. Six native speakers produced accented and unaccented words that varied in segmental type of the word-medial mora $(N=864)$. It was found that the pitch-accent contrast was produced differently in words with a short vowel in the first syllable, CVCVCV and CVC:V words (V-group), on one hand, and words with a longer voiced portion of the first syllable, $\mathrm{CV}: \mathrm{CV}$ and $\mathrm{CVNCV}$ words (V:/VN-group), on the other hand, $p>0.01$. In the accented condition, the average $F 0$ fall was $50 \mathrm{~Hz}$ for all, but the $F 0$ movement within the first syllable was different between the two word groups. In the unaccented condition, word-level, as well as intrasyllabic differences, were observed in $F 0$ contours. $F 0$ was rising in the V-group, whereas it was flat in the V:/VN-group. These results indicated that pitch patterns varied by segmental structure, more so for unaccented words.

2aSC14. Perception of Japanese pitch accent: Examination of minimal accent pairs. Yukiko Sugiyama (Dept. of Linguist., Univ. at Buffalo, SUNY, 609 Baldy Hall, Buffalo, NY 14260, ys8@buffalo.edu)

Perception of Japanese pitch accent was examined using 20 bimoraic/ disyllabic minimal accent pairs. A minimal accent pair refers to a pair of words that is identical except for their accent types. For example, /hana/ "flower" and /hana/ "nose" have the same phonemic sequence and the pitch pattern of low-high yet they differ in that "flower" has accent on the word-final mora while "nose" has no accent (unaccented). A preceding production study that utilized the same 20 pairs of words found that the maximum F0 in the word-final mora was reliably higher for the finalaccented words than the unaccented words and the F0 rise from the initial mora to the final mora was reliably larger for the final accented words than the unaccented ones when they were produced sentence-medially followed by a particle. However, the two accent types did not show any reliable difference when they were produced by themselves in isolation. This perception study accesses if the properties found to be related to Japanese pitch accent in production are actually used by listeners when they identify accent types. The relation between the production and perception of Japanese pitch accent will be discussed.

2aSC15. The phonetic and phonological triggers for downstep in Japanese: Evidence from perception. Takahito Shinya (Dept. of Linguist., 226 South College, Univ. of Massachusetts, Amherst, MA 01003, tshinya@linguist.umass.edu)

In Japanese the F0 peak of a word is downstepped after a lexically accented word. Lexical accent can be characterized by its phonological specification in the lexicon or by its phonetic F0 pattern. A perceptual experiment was conducted in order to observe how these two properties interact with each other as downstep triggers. The experiment tested whether listeners compensate for downstep exclusively based on the phonological information of lexical accent or on the phonetic cues available. Participants were asked to judge the relative prominence between two F0 peaks. Results suggest that in order for two words to be perceptually equal in prominence, the second F0 peak must be lower than a given first F0 peak. This difference between the two F0 peaks was significantly greater when the first word was phonetically accented than when it was phonetically unaccented, which suggests that the downstepped F0 peak is perceptually compensated. A similar effect was observed for the phonologically accented first peak, but the effect size was much smaller than the effect observed for the phonetically accented first peak. These results suggest that the primary trigger of downstep in Japanese is the F0 pattern of accented words. Lexical specification plays, at best, a secondary role. 
2aSC16. Pitch values in prominent and nonprominent sentence enders in Japanese. Yumiko Enyo (Dept. of Linguist., Univ. of Hawaii at Manoa, 1890 East-West Rd., Honolulu, HI 96822)

This study uses naturally occurring interview data to investigate properties of intonational prominence in Japanese sentence enders (SEs). Since Japanese intonation patterns generally observe downstep (Venditti, 2005), a sentence-ending AP (accentual phrase) is likely to have the lowest F0 in an utterance if it does not receive prominence. Data from one interviewee's responses, SEs in long IPs (more than 5 APs) and short IPs (one or two APs), are used for this analysis. Within the group of 28 short IPs, there were eight cases of the SE nandaroo "what is it, I wonder." Measurements of highest and lowest F0 within nandaroo indicate two distinctive F0 patterns, determined by whether the SE is prominent or nonprominent. (Prominence appears to be placed on an SE when its content is focused.) In prominent SEs, the intonation contour contains peaks that exceed three standard deviations of the speakers F0 average. However, prominent and nonprominent utterances are similar in their F0 minima. As a result, SEs with prominence typically show wider pitch range than those without prominence. Similar results are found for other SEs contained in both long and short IPs.

2aSC17. On the prosody control characteristics of nonverbal utterances and its application to communicative prosody generation. $\mathrm{Ke} \mathrm{Li}$, Yoko Greenberg, Nagisa Shibuya, Yoshinori Sagisaka (GITI Waseda Univ. 29-7 Bldg. 1-3-10 Nishi-Waseda, Shinjuku-ku, Tokyo 169-0051, Japan,rikoku@akane.waseda.jp), and Nick Campbell (NiCT/ ATR)

In this paper, prosodic characteristics of nonverbal utterances were analyzed using an $F 0$ generation model proposed by Fujisaki aiming at communicative speech generation. From the analysis, the different distributions of $F 0$ generation parameters have been observed for prototypical four dynamic patterns (rise, gradual fall, fall, and rise\&down). Since former works have shown that these differences can correspond to their impressions (such as confident-doubtful, allowable-unacceptable, and positive-negative) expressed by multi-dimensional vectors, we tried to make a computational model from impression vector to $F 0$ generation parameters. By employing a statistical optimization technique, we have achieved the mapping from impression vectors to prosody generation parameters. Perceptual evaluation tests using neutral words have confirmed the effectiveness of the mapping to provide communicative speech prosody. [Work supported in part by Waseda Univ. RISE research project of "Analysis and modeling of human mechanism in speech and language processing" and Grant-in-Aid for Scientific Research B-2, No. 18300063 of JSPS.]

2aSC18. Analyses of fundamental frequency contours for Japanese utterances that express several degrees of four emotions-joy, sadness, anger, and fear. Hiromi Kawatsu and Sumio Ohno (Grad. School of Bionics, Comput. and Media Sci., Tokyo Univ. of Technol., 1404-1 Katakura, Hachioji, Tokyo, 192-0982, Japan, kawatsu@so.cs.teu.ac.jp)

To give synthetic speech richer expression, prosodic features of utterances with various kinds of emotions were analyzed. Utterances that express four basic emotions with several degrees were collected as speech material: joy, sadness, anger, and fear. The fundamental frequency contours are analyzed based on a model for the process of generation. Changes in controlling parameters of the model were examined with regard to degrees of emotion. The baseline frequency increases as degrees of emotion increase. Especially for sadness and anger, its tendency is remarkable. Regarding phrase commands, the rate of occurrence increases as the respective degrees of emotion increase. The rates are affected by the kind of branch boundary in the grammatical structure and the number of morae from the immediately preceding phrase command. The change of the amplitude of phrase commands depends on the kind of position of grammatical structure. For the accent commands, timings of their onsets and offsets are almost constant for degrees of emotion. They depend on the accent types of prosodic words. The magnitude of the accent commands changes as degrees of emotion increase depending on the positions of prosodic words from the beginning of the utterance.

2aSC19. An acoustic functional grammar of English intonation. Hunter B. Hatfield (Univ. of Hawaii, 438 Launiu St., Apt. A, Honolulu, HI 96815, hunterh@hawaii.edu)

There has been a resurgence in recent years of linguistic models that directly embed exact physical and acoustic data right into the formalism. This work attempts to show that many aspects of speech perception and production can only be understood when the acoustic input is fully considered. In the case of speech perception, if there are abstractions, they are abstractions over acoustic input, not based on a priori phonological categories. The current paper extends this work into English intonation. The functional phonology of Paul Boersma, which incorporates exact frequency values into an optimality theoretic perception grammar, is extended to explain several basic facts about English intonation, including the derivation of categorical high and low tones from the acoustic signal, the relation of tones to hierarchically adjacent tones, and the affectual aspects of classically paralinguistic intonation. The primary advantage of this approach is that it allows a single model to handle both linguistic and paralinguistic intonation structure, a merger which is only possible due to the use of physical acoustic data in the model.

2aSC20. Does English have accent domains? Molly Shilman (Linguist. Dept., UCLA, Los Angeles CA 90095-1543, mshilman@ humnet.ucla.edu)

In ToBI systems, there are languages in which a pitch accent is the head of a particular level of phrase (accentual phrase) (e.g., French, Japanese), and those in which pitch accents are largely independent of phrasing (e.g., English, German). However, some theories of syntax-prosody mapping, such as Truckenbrodt's (1999), assume that a pitch accent is always the head of some prosodic domain. Such theories thus predict that every language with pitch accents should have a prosodic unit that is the domain of pitch accent. Previous work suggests that prosodic boundaries tend to be marked by several types of phonetic cues, including final lengthening (Wightman et al., 1992; Jun, 2005). This study uses a controlled production experiment to determine whether there are accent domains in English: speakers produce near-minimal triplets in which the syntactic relations between words are changed by context but the segmental string, location of word boundaries, and location of the single pitch accent within the target phrase all remain fixed. Productions are then compared for final lengthening at the location of target boundaries. Preliminary results suggest that there is an accent domain in English.

2aSC21. Testing the implicit prosody hypothesis: Data from English. Sun-Ah Jun (Dept. of Linguist., UCLA, Los Angeles, CA 90095-1543, jun@humnet.ucla.edu)

One of the recent theories in sentence processing hypothesized that the prosodic phrasing implicit to a certain syntactic structure determines how speakers interpret the structure. According to this implicit prosody hypothesis (IPH; Fodor, 1998, 2002), in a structure of relative clause (RC) with a complex head noun, speakers/languages tend to prefer low attachment (i.e., an RC is modified by the adjacent head noun) if the prosodic break adjacent to RC is smaller than that between the head nouns. However, speakers/languages tend to prefer high attachment (i.e., an RC is modified by the nonadjacent head noun) if the prosodic break adjacent to RC is greater than that between the head nouns. Jun and Koike (2003) and Jun and Kim (2004) have shown that Japanese and Korean speakers who prefer high attachment produced a bigger prosodic break adjacent to RC than between the head nouns, confirming the IPH. The current study examines the prosodic phrasing of English sentences to investigate whether speakers 
of American English, known to prefer low attachment, would produce a larger prosodic break between the two head nouns than that between the $\mathrm{RC}$ and the adjacent head noun. Data from 30 speakers will be discussed. [Work supported by UCLA Faculty Grants.]

2aSC22. Phrasal length and complexity effects on interphrase pause duration. Jelena Krivokapic (Dept. of Linguist., Univ. of Southern California, 3601 Watt Way, GFS 301, Los Angeles, CA 90089-1693,krivokap@usc.edu)

This study examines the effect of prosodic structure and phrase length on pause duration and the speech planning processes that such pauses can reveal. Subjects read sentences varying in two ways. First, each sentence included a pause bounded either by long intonational phrases both before and after it, medium phrases before and after, or short phrases before and after. Second, the prosodic complexity of the phrase on each side of the pause, i.e., before it and after it, was varied as to whether it was a branching intonational phrase or a nonbranching intonational phrase. Fourteen speakers read sentences synchronously in dyads so as to minimize variability due to speech rate and individual differences [Cummins, ARLO (2002), Cummins, J. Phonetics: (2003); Zvonik and Cummins, ICSLP (2002)]. The results for the dyads analyzed to date indicate that preboundary branching phrases are followed by longer pauses than nonbranching preboundary phrases. Further, surrounding long phrases lead to longer pauses than surrounding medium phrases, which in turn give rise to longer pauses than surrounding short phrases. Interaction effects of phrasal length and complexity will also be discussed. [Work supported by NIH.]

2aSC23. Speech timing: Final lengthening and syllable reduction in cerebellar pathology. Maureen A. Casper and Jennifer Geibel (State Univ. of New York @ New Paltz; 75 South Manheim Blvd., New Paltz, NY 12561)

The production of prosodic prominence may be considered the hallmark of normal speech motor control. Two theoretical views predict different relations between duration and formant and fundamental frequencies. The dynamic view of coordination predicts a disassociation between time and movement amplitude, while the clock theory of cerebellar movement poses a monotonic relation between duration and frequency. Phrasefinal lengthening and syllable reduction each were measured across a prosodic contrast design for the acoustic measures of syllable duration, $F 0$, $F 1$, and $F 2$. The speech characteristics of normal and cerebellar ataxic speakers were compared. In support of gestural dynamics, and following the kinematic findings for normal speakers, the acoustic findings showed longer duration and reduced frequency for phrase-final lengthening, and shorter duration and reduced frequency for syllable reduction. Cerebellar speakers produced longer durations for both events and increased variability for formant and fundamental frequency. This dissimilarity between normal prosodic production and that of cerebellar speakers implies a dynamic relationship between time and articulatory movement. In contrast to normal speech production, cerebellar speech is markedly slow and fails in the rapid adaptation to reduce frequency in phrase-final and reduced syllables.

2aSC24. Voice $F 0$ responses to actual versus consciously perceived auditory feedback pitch shifts. Theresa Burnett and Dana Madonia (Dept. of Speech \& Hearing Sci., Indiana Univ., 200 S. Jordan Ave., Bloomington, IN 47405)

The purpose of this study was to determine the effect of pitch shift discrimination ability on production of voice $F 0$ responses to auditory feedback pitch shifts. Subjects repeatedly vocalized /u/ into a microphone while listening to their voice fed back over headphones. During each trial, auditory feedback pitch was experimentally shifted upward or downward (randomly determined) by 50 cents for $100 \mathrm{~ms}$. Subjects subsequently pressed a button labeled up or down to indicate their perceived direction of voice feedback pitch shift. Voice output was recorded, F0 extracted, smoothed, time aligned to the onset of the pitch shift, and averaged. Button response accuracy was found to be at chance expectancy among the majority of subjects. Nevertheless, all subjects responded vocally to the pitch shifts by changing voice $F 0$ in the opposite direction, indicating that the direction of the shift was indeed processed by the auditory system. These results are consistent with the existence of preattentive mechanisms of audio-vocal control, and provide evidence for a mismatch between conscious and preattentive auditory processing of voice pitch feedback for the control of voice $F 0$. [Work supported by NINDS.]

2aSC25. Correlations between voice $F 0$ responses and manual reaction times to auditory feedback pitch shifts. Dana Madonia and Theresa Burnett (Dept. of Speech \& Hearing Sci., Indiana Univ., 200 S. Jordan Ave., Bloomington, IN 47405)

There appear to be two types of voice $F 0$ responses to voice auditory feedback pitch manipulations: early, opposing responses, and later responses that vary across subject and task. The purpose of this study was to determine whether either type correlates with volitional motor abilities. Subjects vocalized / $\mathrm{u} /$ into a microphone while listening to their voice fed back over headphones. Subjects also pressed a joystick button as rapidly as possible upon hearing any experimenter-generated auditory feedback pitch shift. Pitch shift stimuli were 50 cents upward or downward (randomized), and 100-ms duration. Voice was recorded, F0 extracted, smoothed, time aligned to the onset of the shift, averaged according to shift direction, and the onset of the first voice $F 0$ response was measured. Average manual reaction time was calculated. Findings indicated no significant correlation between manual responses and early voice $F 0$ response. However, later voice $F 0$ responses and manual reaction times were positively correlated. These results are consistent with the hypothesis that the early voice $F 0$ response is automatically generated, and later responses are under voluntary influence. Results also suggest that the variability of later voice $F 0$ responses may be a function of individual differences in volitional motor response abilities. [Work supported by NINDS.]

2aSC26. Frequency and amplitude derivatives as syllable-level $\boldsymbol{F 0}$ features. Mark Liberman and Jiahong Yuan (Univ. of Pennsylvania, Philadelphia, PA 19104)

Most descriptions of tone and intonation focus on the alignment of $F 0$ rises, falls, and inflection points relative to the phonetic unfolding of associated syllables. In this context, it is suggested that the relationship between proportional changes in $F 0$ (treated modularly to eliminate the effects of period doubling and halving) and proportional changes in amplitude (within the frequency bands typically dominated by energy from voicing) provides an interesting characterization of $F 0$ trajectories in relation to syllabic structure. Using data from conversational and broadcast speech in English and Chinese, parameters of this type (in combination with other acoustic measures) are used in identifying relevant features of tone and intonation.

2aSC27. Declination of subglottal pressure during speech and its relation to tone distribution. Helen M. Hanson, Janet Slifka, Stefanie Shattuck-Hufnagel (Speech Commun. Group, Res. Lab. of Electron., MIT, 77 Massachusetts Ave., Cambridge, MA 02139), and James B. Kobler (Massachusetts General Hospital, Boston, MA 02114)

The subglottal pressure contour $P_{s}$ for speech is considered to have three phases: initiation (rapid rise), working (level or slightly declining), and termination (rapid fall). The current work focuses on characterization of the working phase in terms of the distribution of pitch accents and of phrase and boundary tones. In particular, the degree of $P_{s}$ declination is studied. A measure of $P_{s}$ declination has proven difficult to define [cf. Strik and Boves, J. Phonetics 23, 203-220 (1995)]. Therefore, in pilot work, subjective ratings of degree of declination are made on a subset of 
a corpus in which the tone distribution is controlled. Significant variation in the degree of declination is observed among speakers. For example, $P_{s}$ for one speaker is relatively constant to slightly declining, while for another it is almost always sharply declining. For some speakers utterances with an early-occurring nuclear pitch accent (NPA) show greater degree of declination than utterances with a late-occurring NPA, as do utterances ending with high tones. Data for additional subjects and objective measures will be presented. The results have implications for models of speech production, and for applications such as computer speech synthesis and recognition. [Work supported by NIH Grants DC00075 and DC04331.]

2aSC28. Comparing methods for measuring pitch "elbows." Ryan Shosted, ${ }^{a}$ Alex Del Giudice, and Amalia Arvaniti (Dept. of Linguist., Univ. of California, San Diego, 9500 Gilman Dr., \#0108, La Jolla, CA 92093)

Currently, there is no standard method for determining the location where an F0 curve begins its upward or downward trajectory (sometimes referred to as the pitch "elbow"). It is generally accepted that elbows are hard to measure in a consistent way: some studies have used least-squares fitting methods, others have relied on visual judgments. This study uses a variety of data from English and Greek that exhibit different degrees of difficulty for locating the elbow. Automated results are derived from various implementations of two algorithms. The first algorithm uses linear least-squares fitting, where the intersection of two lines that best approximate the pitch contour denotes the point of the elbow. The second algorithm analyzes point-by-point changes. The results are then compared with those of human labelers. We present results from a series of statistical tests that estimate the robustness of variations on these two algorithms with respect to the performance of the human labelers. Our aim is to provide a method for measuring elbows that is robust and easy to implement and that can be used with different types of pitch contours, in order to improve accuracy of measurements. ${ }^{\text {a) }}$ Also at Univeristy of California, Berkeley.

2aSC29. Semi-automatic labeling of pitch accents in American English. Diana Stojanovic and Yohei Sakata (Dept. of Linguist., Univ. of Hawaii, 1890 East-West Rd., Honolulu, HI 96822)

This study provides a simple classification of several pitch accents that occur in American English, based on acoustic measurements of F0. Audio samples recorded by a female speaker and transcribed in the ToBI framework (Beckman, 1994) were used to develop five rules to recognize the pitch accents $\mathrm{H}^{*}, \mathrm{~L}^{*}, \mathrm{~L}+\mathrm{H}^{*}$, and $\mathrm{L}^{*}+\mathrm{H}$. Since no information about prosodic phrasing was given to the recognizer, some combinations with phrase accents were considered and contours then grouped into equivalent labels (e.g., \%HL* equivalent to $\mathrm{H}^{*} \mathrm{~L}-$ ). Based on the rules developed, a semi-automatic process of labeling pitch accents was implemented in Praat. Half of the audio samples were used to train the labeler, the other half to test it. The accuracy level achieved was $69 \%$. When effects of microprosody, creaky voice, or incorrect automatic pitch tracking were removed, the accuracy of classification into equivalent labels increased to $90 \%$. Two types of applications are proposed: semi-automatic computer labeling of intonation for larger quantities of data, and a tool to help human transcribers learn American English ToBI using Praat. In addition, the simple classification is the first step towards looking into speaker variability in realization of different contours, and typology of intonational contours in different languages.

2aSC30. Parametric spectrogram modeling of single and concurrent speech with spline pitch contour. Jonathan Le Roux, Hirokazu Kameoka, Nobutaka Ono, and Shigeki Sagayama (Grad. School of Information, Phys. and Computing, The Univ. of Tokyo. 7-3-1, Hongo, Bunkyo-ku 113-8656 Tokyo, Japan)

This paper proposes a novel general speech analysis framework consisting in a precise and parametric description of speech directly from the power spectrum, resulting in a new efficient pitch estimation method for both single and multiple speaker situations. The speech spectrum is mod- eled as a sequence of spectral clusters governed by a common pitch contour expressed as a spline curve. These clusters are isolated by an unsupervised 2-D time-frequency categorization of the energy density, and their common pitch contour is estimated simultaneously. A smooth pitch contour can thus be extracted on the whole utterance, linking together its voiced parts, while this is difficult or impossible for frame-by-frame algorithms. The proposed algorithm is compared to several existing methods on a database of single speaker speech, with very good results that place it among the most accurate ones. Moreover, its very high accuracy is shown on the estimation of multi-pitch contours of co-channel concurrent speech.

2aSC31. Robust $\boldsymbol{F 0}$ estimation based on complex speech analysis. Tatsuhiko Kinjo and Keiichi Funaki (Univ. of the Ryukyus, Okinawa, Japan)

This paper proposes a novel robust fundamental frequency $(F 0)$ estimation algorithm based on complex-valued speech analysis for an analytic speech signal. Since the analytic signal provides spectra only over positive frequencies, spectra can be estimated accurately at low frequencies. Consequently, it is considered that $F 0$ estimation using the residual signal extracted by complex-valued speech analysis can perform better for $F 0$ estimation than that for the residual signal extracted by conventional realvalued LPC analysis. In this paper, the autocorrelation function (AUTOC) weighted by a reciprocal of the AMDF is also adopted for the $F 0$ estimation criterion. The proposed $F 0$ estimation algorithm is evaluated using three criteria, AUTOC, AMDF, and the weighted AUTOC, with complexvalued residual extracted by complex-valued speech analysis. We also compared the proposed method with those for three signals: the speech signal, analytic speech signal, and LPC residual. Speech signals used in the experiments were corrupted by addition of white Gaussian noise whose noise levels are $10,5,0$, and $-5[\mathrm{~dB}]$. The performance was evaluated using the gross pitch error (GPE) and fine pitch error (FPE). The results demonstrate that this algorithm based on complex speech analysis can perform better than conventional methods in an extremely noisy environment.

2aSC32. Cross-linguistic examination of listeners frequency thresholds for musical tones and vowels. Daniel Pape (ZAS Berlin, Jgerstrasse 10-11, 10117 Berlin, Germany)

There is clear evidence that musical education increases the discriminability of the frequency threshold (dl). If musical education increases sensitivity, the question arises if listeners of a language that uses F0 for prosody are more sensitive to musical tones and vowels' dl thresholds. A perception experiment was conducted examining the dl of musical tones and vowels for both professional musicians and nonmusicians. The experiment was carried out cross linguistically: The languages include German and English (Germanic languages) and Italian, Portuguese, and Spanish (Romance languages). Results indicate that $\mathrm{dl}$ is significantly smaller for musicians compared to nonmusicians for all examined languages. The native vowel $\mathrm{dl}$ is significantly smaller for the Germanic languages. This is attributed to the more frequent use of F0 for prosody, thus claiming linguistic reasons. Surprisingly the $\mathrm{dl}$ for the musical tone in all languages is smaller for the Germanic musicians compared to the Romance musicians, although musical education and the stimulus were identical for all musically educated musicians. These results indicate an influence of the native language on the pitch accuracy of the listeners. Thus we give evidence that interference of speech pitch and musical pitch processing occurs, which supports the "common processing theory."

2aSC33. Peak alignment in accented Tolowa syllables. Christopher Doty (Dept. of Linguist., Univ. of Oregon, 1290 Univ. of Oregon, Eugene, OR 97403-1290, suomichris@gmail.com)

Tolowa (Athabaskan, Pacific Coast Group) has only three remaining fluent speakers near Crescent City, CA. Tolowa has prominent pitch contours that have been described both as an interaction of stress and vowel length and as lexically specified pitch accent. The present study examined 
the phonetic realization of these pitch contours. Fundamental frequency $(F 0)$ at its highest peak during the rhyme of accented syllables was measured, as was relative duration into the rhyme at which the peak occurred. Preliminary results indicated that a high pitch peak occurred approximately $20 \%-30 \%$ of the way into the rhyme of the syllable. In the case of long vowels in accented syllables, this led to a prominent peak which fell sharply during the second half of the rhyme. A similar peak and fall was seen in accented syllables closed by a voiced sonorant. In the case of accented syllables with short vowels closed by a voiceless consonant, the accent was realized as a rise in pitch leading to the final consonant. These results indicate that the phonetic target is a high peak $20 \%-30 \%$ into the rhyme; the target seems to not include the fall seen in rhymes with long vowels or those closed by voiced sonorants.

2aSC34. Production characteristics of accent in four dialects of Nahuatl. Susan G. Guion (Linguist. Dept., Univ. of Oregon, 1290 Univ. of Oregon, Eugene, OR 97403, guion@uoregon.edu), Jonathan D. Amith (Gettysburg College, Gettysburg, PA 17325), Christopher Doty, and Irina A. Shport (Univ. of Oregon, Eugene, OR 97403)

Most descriptions of Nahuatl (Uto-Aztecan, Mexico) describe regular penultimate accent, although production characteristics are unstudied. Recently, however, dialects from Guerrero state have been described with pitch-accent from historical coda breathy $/ \mathrm{h} /$ in nonfinal position [Guion and Amith, J. Acoust. Soc. Am. 117, S2490 (2005)]. In the current study, the acoustic correlates of this new pitch-accent, as well as penultimate accent, are investigated. Speakers $(n=6)$ from four dialects-two with innovated pitch-accent and two with only penultimate accent-were recorded. The production characteristics of fundamental frequency (F0), duration, and voice quality, as indexed by the difference between the first two harmonics (H1-H2), were investigated. Innovated pitch-accent was found to be cued solely by F0. Penultimate accent varied in its production by dialect. In dialects with only penultimate accent, production exhibited characteristics typical of stress-accent: Accented syllables had higher F0, longer durations, and more vocal effort (smaller H1-H2 differences) than unaccented syllables. Dialects with innovated pitch-accent had less stresslike penultimate accents, being cued primarily by F0, or with a relatively small durational difference, but no voice quality difference. This indicates that the innovation of a pitch-accent has changed the formerly stresslike penultimate accent into a more pitch-type accent.

2aSC35. Lexical stress without postlexical head-marking: Evidence from Tagalog. Victoria B. Anderson (Dept. of Linguist., Univ. of Hawaii, 1890 East-West Rd., Honolulu, HI 96822, vanderso@ hawaii.edu)

Jun (2005) compares prosodic prominence types in 21 languages representing diverse families (including Australian, Chinese, Germanic, Muskogean, Romance, and Semitic, but none from Austronesian). Jun observes an implicational relationship between the prominence type a language uses at the lexical level (i.e., lexical tone, stress or pitch accent) and the prominence type it uses postlexically (i.e., head-marking or edgemarking). Fourteen languages in the sample employ lexical stress; all use postlexical head-marking (i.e., postlexical pitch accent on a phrases most prominent syllable), and four additionally mark a phrases' edge(s). No stress language in the sample employs postlexical edge-marking alone. However, Tagalog may be such a language. Tagalog uses lexical stress (LAyas "leave;" laYAS “carefree"), but arguably does not use headmarking. This study examined map-task data from four native speakers of Tagalog. Syllables bearing main stress in a phrase, and occurring between zero and three syllables from the end of the phrase, were analyzed for pitch, intensity, and duration. Results show that although syllables with primary stress have increased intensity, and duration, pitch prominences do not reliably align with these syllables. Instead, low pitch reliably appears on the first syllable in an accentual phrase, while high pitch reliably appears on the last syllable.

2aSC36. Bengali intonational phonology. Sameer ud Dowla Khan (Dept. of Linguist., UC Los Angeles, 3125 Campbell Hall, UCLA, Los Angeles, CA 90095-1543)

Previous studies of Bengali intonation are primarily concerned with the mapping between syntax and intonation, the existence of accentual and phonological phrases, optionality in phrasing, and realization of focus. While these studies have been influential, they shy away from a full description of Bengali intonation, drawing from a small data set produced by a handful of speakers. At present, there is no comprehensive analysis of Bengali intonation that uses data collected from a wide range of speakers. The current study adopts the framework of intonational phonology (Pierrehumbert, 1980; Ladd, 1996) to develop such an analysis based on a large set of data from approximately 25 Bengali speakers. A pilot study measuring pitch and segment durations in 155 sentences suggests the existence of three pitch accent types (low, high, rising) and three levels of prosodic units whose right edges are marked by a boundary tone (intonation, intermediate, accentual). This suggests that Bengali differs from most other languages (Jun, 2005) in having both (1) contrastive pitch accents and (2) a small prosodic unit that is the domain of the pitch accent (i.e., the accentual phrase). Further analyses will illustrate the phonetic realizations of pitch accents and boundary tones in the language.

2aSC37. Prosodic correlates to meaning in infant-directed speech. Debora S. Herold (Dept. of Psych., Indiana Univ. Purdue Univ. at Indianapolis, 402 North Blackford St., Indianapolis, IN 46202, dssasso@iupui.edu), Laura L. Namy, and Lynne C. Nygaard (Emory Univ., Atlanta, GA 30322)

The present investigation examined the extent to which speakers produce reliable prosodic correlates to meaning across a range of semantic domains and whether parents use these kinds of cues to teach word meanings to children. In study 1 , acoustic properties including fundamental frequency $\left(F_{0}\right), F_{0}$ variation, duration, and amplitude of infant-directed utterances were examined. Speakers were asked to produce question phrases in which novel words were used to convey one of two meanings from a set of antonym pairs (e.g., big/small, yummy/yucky). Herold (2006) has shown that adult listeners can identify word meanings from the prosodic information contained in these utterances. Acoustic analyses revealed that each word meaning had a unique acoustic signature, and that related word meanings such as big and tall and small and short had similar acoustic profiles. Study 2 explored whether naturalistic parental speech to young children recruits similar acoustic properties in the service of providing prosodic cues to meaning. Results demonstrated that mothers systematically varied prosodic cues as a function of word meaning when producing speech to their children. These findings suggest that speech directed to infants contains reliable prosodic markers to word meaning and that mothers may use prosodic cues to differentiate word meanings in word learning contexts.

2aSC38. Influence of voice prosody on preferential behaviors. Naoki Hasui and Yugo Takeuchi (1-39-18 Sumiyoshi, Hamamatsu-shi, Shizuoka, 432-8011 Japan, gs06044@s.inf.shizuoka.ac.jp)

In mother-infant interactions, the mother talks in a special voice that is characterized by elevated fundamental frequency (pitch) and exaggerated intonation contours, and the infant turns a face toward the voice and smiles naturally. This case suggests that humans react automatically to the special prosody without understanding of the lexical meaning in the word. We propose a hypothesis that adults react automatically as in the case of the infant by not only a difference of prosody but also the situation that one is in. This study aims to figure out the function of prosody on voice 
cognition and utilize these findings for constructing the voice user interface systems with a low cognitive load for users. In this paper, using the virtual maze on a display, we gave the participants a task that they should escape as quickly as possible from the maze. In the situation where the participants choose the route on T-shaped intersection, we gave the participants two leading voices of different prosody, and observed the preferential behavior for the two voices. The experimental results indicate that the voice of the voice user interface systems synthesizing the high pitch or the high rate of speech is naturally accepted and can direct the users.

2aSC39. Utilization of acoustic cues in identifying speech intonation contrasts in cochlear implant recipients and listeners with normal hearing. Shu-Chen Peng (Dept. of Hearing and Speech Sci., Univ. of Maryland, College Park, MD 20742)

This study investigated the utilization of acoustic cues in identifying speech intonation contrasts in adult cochlear implant $(\mathrm{CI})$ recipients and normal-hearing $(\mathrm{NH})$ individuals listening to spectrally and temporally degraded stimuli. Fundamental frequency, intensity, and duration patterns of a disyllabic word, popcorn, were manipulated orthogonally, resulting in 360 resynthesized stimuli. In a two-alternative forced-choice task, CI and $\mathrm{NH}$ participants identified whether each stimulus was question-like or statement-like. Each $\mathrm{NH}$ listener also identified stimuli that were noise vocoded. Preliminary results from seven CI and four NH listeners indicated that (a) weighting of fundamental frequency in the identification of $\mathrm{CI}$ listeners was less pronounced than that of $\mathrm{NH}$ listeners, (b) unlike $\mathrm{NH}$ listeners who showed little reliance upon intensity or duration patterns in identifying unprocessed stimuli, CI users demonstrated systematic dependence upon intensity and duration cues in identifying the same set of stimuli, and (c) with noise-vocoded (spectrally and temporally degraded) stimuli, NH listeners' identification exhibited weighting patterns for inten- sity and duration cues in a way similar to that of CI listeners. Implications for the processing of multidimensional acoustic cues for speech intonation in normal and electrical hearing will be discussed. [Work supported by NIDCD-R01DC04786.]

2aSC40. The voices of anger and disgust: Acoustic correlates in three languages. Marc D. Pell, Areej Alasseri (School of Commun. Sci. \& Disord., McGill Univ., 1266 Pine Ave. West, Montreal, QC H3G 1A8, Canada, marc.pell@mcgill.ca), Sonja A. Kotz, and Silke Paulmann (Max Planck Inst. for Human Cognit. and Brain Sci., 04103 Leipzig, Germany)

Anger and disgust are believed to represent discrete human emotions with unique vocal signatures in spoken language. However, few investigations describe the acoustic dimensions of these vocal expressions, and some researchers have questioned whether disgust is robustly encoded in the vocal channel. This study sought to isolate acoustic parameters that differentiate utterances identified as sounding angry versus disgusted by listeners based on evidence from three separate languages: English, German, and Arabic. Two male and two female speakers of each language produced a list of pseudo-sentences (e.g., Suh fector egzullin tuh boshent) to convey a set of seven different emotions. The recordings were later judged by a group of native listeners to determine what emotional meaning was perceived from the prosodic features of each pseudo-utterance. Individual sentences identified systematically as conveying either anger or disgust (greater than $3 \times$ chance target recognition) were then analyzed acoustically for various parameters of fundamental frequency, amplitude, and duration. Analyses compared which acoustic parameter(s) were dominant for identifying anger versus disgust in each language, and whether these patterns appeared to vary across languages, with implications for understanding the specificity and universality of these emotion expressions in the vocal channel.

\title{
Session 2aSP
}

\section{Signal Processing in Acoustics, Architectural Acoustics, Engineering Acoustics, Psychological and Physiological Acoustics: Spatial Sound Processing, Control, and Performance Evaluation}

\author{
William L. Martens, Cochair \\ McGill Univ., Faculty of Music, 555 Sherbrooke St. W., Montreal, Quebec, H3A 1E3, Canada \\ Kazuhiro Iida, Cochair \\ Matsushita Electric Industrial Co., Ltd., AV Core Technology Development Ctr., 600 Saedo, Tsuzuki, \\ Yokohama 224-8539, Japan
}

Chair's Introduction-8:00

Invited Papers

8:05

2aSP1. Median plane localization using a parametric model of the head-related transfer function based on spectral cues. Kazuhiro Iida, Motokuni Itoh (AV Core Technol. Development Ctr., Matsushita Electric Ind. Co., Ltd. 600 Saedo, Tsuzuki, Yokohama, 224-8539 Japan, iida.kazuhiro@jp.panasonic.com), Atsue Itagaki, and Masayuki Morimoto (Kobe Univ. Rokko, Kobe, 657-8501 Japan)

The present study has two purposes. One is to examine a simulation method for localizing a sound image. The authors propose a parametric HRTF model to simulate vertical sound localization. The parametric HRTF is recomposed only of the spectral peaks and notches extracted from the measured HRTF, and the spectral peaks and notches are expressed parametrically with center frequency, level, and sharpness. The other purpose is to clarify the contribution of each spectral peak and notch as a spectral cue. Localization tests were carried out in the upper median plane using the subjects' own measured HRTFs and the parametric HRTFs with various combinations of spectral peaks and notches. The results show that the parametric HRTF recomposed using the first and second notches 
(N1 and N2) and the first peak (P1) provides almost the same localization accuracy as the measured HRTFs. Observations of the spectral peaks and notches indicate that N1 and N2 change remarkably as the source elevation changes, whereas P1 does not depend on the source elevation. In conclusion, N1 and N2 can be regarded as spectral cues, and the hearing system could utilize P1 as the reference information to analyze $\mathrm{N} 1$ and $\mathrm{N} 2$ in ear-input signals.

2aSP2. Evaluation of head-related transfer function on the opposite side to a sound source. Takanori Nishino (Ctr. for Information Media Studies, Nagoya Univ., Nagoya, Aichi, 464-8603 Japan), Takayuki Oda, and Kazuya Takeda (Nagoya Univ., Nagoya, Aichi, 464-8603 Japan)

A head-related transfer function (HRTF) is a complex acoustic transfer function because it includes the effect of reflection and refraction due to the head and ears. The HRTFs on the opposite side to a sound source have more complex characteristics and lower power than HRTFs that are on the same side as a sound source. Moreover, many previous studies about monaural sound localization showed that performances of sound localization on the opposite side were not precise. From these results, it can be assumed that the HRTFs on the opposite side are not necessary to use listener's own. In our experiments, several stimuli that convolved with the HRTFs were presented to evaluate individuality. The left ear HRTF was the subject's own, and the right ear HRTF was the subject's own and others. The differences between the subject's HRTF and others' HRTFs were evaluated using the spectral distortion score. The sound source azimuths were from 0 to $180 \mathrm{deg}$ at intervals of $30 \mathrm{deg}$; the front was $0 \mathrm{deg}$, the left side was $90 \mathrm{deg}$, and the back was 180 deg. The results shows that the right ear HRTFs between 30 and 150 deg azimuth have low individualities.

\section{8:45}

2aSP3. Classification head-related transfer functions for an individualization procedure by a tournament-style listening-test. Yukio Iwaya and Yôiti Suzuki (Res. Inst. of Elec. Commun., Tohoku Univ., 2-1-1 Katahira, Aoba-ku, Sendai, 980-8577, Japan)

Individualization of head-related transfer functions (HRTFs) is necessary for high-accuracy sound image localization in threedimensional spatial rendering by virtual auditory displays (VADs). However, measurement of HRTFs for each listener for all soundsource directions requires a special measuring apparatus and a long measurement time with a listener's physical load. The authors have therefore proposed an individualization method of HRTFs called Determination method of OptimuM Impulse-response by Sound Orientation (DOMISO), in which the best set of HRTFs is selected using a tournament-style listening test without any measurement. In DOMISO, our HRTF corpus, including more than 120 sets of measured HRTFs, is used. In this study, a classification method of these HRIRs is examined to select good seeds for the tournament. First, relationships among various parameters of HRTFs and individualization results by DOMISO are analyzed using multiple-correlation analysis. Second, using promising parameters, all sets of HRTFs in the corpus are classified as a structured tree. Third, using results of classification, listening tests by DOMISO procedure are performed using these HTRFs. Finally, a listening test of sound image localization is conducted with the individualized HRTFs to examine the effectiveness of the classification.

\section{9:05}

2aSP4. Development of the fast measurement system for the listener's own head-related impulse responses. Kimitoshi Fukudome (Dept. of Acoust. Design, Faculty of Design, Kyushu Univ., Shiobaru 4-9-1, Minami-ku, Fukuoka, 815 Japan, fukudome@design.kyushu-u.ac.jp), Yusuke Tashiro (Kyushu Inst. of Design, Minami-ku, Fukuoka, 815-8540 Japan), Kazuki Takenouchi, Toshiya Samejima, and Naoki Ono (Kyushu Univ., Minami-ku, Fukuoka, 815-8540 Japan)

Three-dimensional (3-D) spacial sound reproduction through headphones or earphones is best realized for subjects by the use of their own head-related impulse responses (HRIRs). Although HRIR measurements are so extremely time consuming, better externalized HRIRs for subjects must be collected in order to develop future 3-D applications. The fast measurement system for the listener's own HRIRs was constructed based on the continuous measurement method with a subject sitting on a servo-swiveled chair (SSC), and together with a loud speaker elevation arm controlled by a stepping motor. It can greatly reduce the subject burden in HRIR measurements. HRIRs for all azimuthal directions at 29 elevations can be measured in $1 \mathrm{~h}$, constituting a reduction by a factor of 3 of the amount of time required as compared to systems composed of an SSC and a conventional manual elevation system. In the presentation, principles of the fast measurement system and the specifications of the loud speaker elevation arm are described. Performance evaluations are presented in terms of the measurement accuracies by the signal-to-deviation ratio and the spectral distortion, and further the reproducibility in repetitive measurements. [Work supported by the specific project research in the Faculty of Design, 2005.]

2aSP5. Capture and rendering of spatial sound over headphones. Ramani Duraiswami, Dmitry N. Zotkin, and Adam O’Donovan (Perceptual Interfaces and Reality Lab., Inst. for Adv. Comput. Studies, Univ. of Maryland, College Park, MD 20742)

A theory for capturing an audio scene and then rendering it remotely over headphones is developed. The method relies on capture of a sound field up to a certain order in terms of spherical wave functions. Then, the captured sound field is convolved with the head-related transfer function and rendered to provide presence in the auditory scene. The sound-field representation is then transmitted to a remote location for immediate rendering or stored for later use. A system that implements the capture using a spherical array is developed and tested. Head-related transfer functions are measured using the system described in [D.N. Zotkin et al., J. Acoustic. Soc. Am. (to appear)]. The sound renderer, coupled with the head tracker, reconstructs the acoustic field using individualized head-related transfer functions to preserve the perceptual spatial structure of the audio scene. [Work partially supported by VA.] 
2aSP6. Acoustic rendering of a virtual environment based on virtual microphone control and binaural room scanning. Jonas Braasch (School of Architecture, Rensselaer Polytechnic Inst., 110 Eighth St., Troy NY 12180), William L. Martens, and Wieslaw Woszczyk (McGill Univ., Montreal, QC H3A 1E3, Canada)

Binaural room scanning (BRS) is a convolution technique that utilizes a set of spatially indexed impulse responses that are selected in response to listener head movements during virtual acoustic rendering, such that the direction of sonic elements is automatically updated according to the angle determined by a head tracker. Since the room impulse responses have to be measured for only a few loudspeaker positions, a very successful application of BRS is the simulation of a control room. In order to create a flexible headphone-based virtual environment, it is proposed to simulate the input signals for the BRS system using virtual microphone control (ViMiC). ViMiC renders a virtual environment by simulating the output signals of virtual microphones that can be used to address a surround loudspeaker system. The advantages of this approach are twofold. First, the measured impulse responses of the BRS system ensure high spatial density in overall reflection patterns, avoiding the typical gaps in between the early reflections that occur when using solely mirror images or ray tracing. Second, the resulting imaging is closer to common audio engineering practice, where a pleasant but plausible environment is favored over a more purely realistic rendering.

10:05-10:20 Break

\section{$10: 20$}

2aSP7. A comparison of listener loudspeaker preference ratings based on in situ versus auralized presentations of the loudspeakers. Sean Olive, Todd Welti (Harman Intl. Industries, Inc., 8500 Balboa Blvd., Northridge, CA 91329), and William Martens (McGill Univ., Montreal, PQ, H3A 1E3, Canada)

Auralization methods have several practical and methodological advantages for studying the perception of loudspeaker reproduction in rooms. For subjective measurements of loudspeakers, the auralization should be capable of eliciting the same sound quality ratings as those measured using an original acoustic presentation of a loudspeaker. An experiment was designed to test whether this is possible. Listeners gave preference ratings for both acoustic (in situ) and auralized double-blind presentations of four different loudspeakers, and the results were compared. The auralized presentations were generated from binaural room-scanned impulse responses of the loudspeakers convolved with the music test signals and presented over headphones equipped with a head-tracking device. For both in situ and auralized methods nine trained listeners gave preference ratings for four different loudspeakers using four different programs with one repeat (eight trials in total). The results show that the auralized and in situ presentations generally produced similar loudspeaker preference ratings.

\section{Contributed Papers}

\section{0:40}

2aSP8. Equalization of a one-dimensional sound field with a movable source. Xi Chen, Timothy W. Leishman, Scott D. Sommerfeldt, and Benjamin M. Faber (Acoust. Res. Group, Dept. of Phys. and Astron., Brigham Young Univ., Provo, UT 84602)

An experimental plane-wave tube with a movable side-branch loudspeaker was used to study simultaneous source and sound field equalization. The effects of equalization and inverse filtering were evaluated for different measured field quantities. Parametric equalization filters were designed based on acoustic pressure and total energy density at a point within the tube. An adaptive filter based on total energy density and the filtered- $x$ algorithm was also implemented. The results of the equalization methods are compared and their advantages and disadvantages are explained.

\section{$10: 55$}

2aSP9. Numerical calculation method of binaural signals suitable for auralization of a reflective sound field. Makoto Otani (Dept. of Intelligent Systems Design Eng., Toyama PRef. Univ., Imizu, Toyama 939-0398, Japan, otani@pu-toyama.ac.jp) and Shiro Ise (Kyoto Univ., Yoshida-Honmachi, Sakyo-ku, Kyoto City 606-8501, Japan)

Based on the Kirchhoff-Helmholtz integral equation, a numerical calculation method, which enables a wave-theory-based auralization of a sound field inside room, is proposed. Binaural signals are synthesized from pressures and particle velocities on the boundary surface and corresponding head-related transfer functions (HRTF). The proposed method can shorten the extra computation time that is required because of changes in numerical conditions caused by head movement and rotation. The boundary element method (BEM) was used to calculate surface values on sound-reflective objects. In addition, HRTFs for various source positions were calculated using a BEM-based HRTF calculation system [M. Otani and S. Ise, J. Acoust. Soc. Am. 119, 2589-2598 (2006)]. Results obtained using the proposed method are compared with those of the conventional BEM, whose boundary consists of a head and a wall, and an image source method, which does not incorporate sound wave motion. Those comparisons reveal that the proposed method can accurately simulate binaural signals, including indirect sound from reflective objects. In addition, interactions between the head and wall are discussed, which are omitted in any auralization method using HRTFs to synthesize binaural signals and have not been investigated.

\section{1:10}

2aSP10. A new digital module for variable acoustics and wave field synthesis. Diemer de Vries, Jasper van Dorp Schuitman (Lab. of Acoust. Imaging and Sound Control, Delft Univ. of Technol., P.O. Box 5046, 2600 GA Delft, The Netherlands), and At van den Heuvel (Acoust. Control Systems B.V., 3886 ZG Garderen, The Netherlands)

In the 1980s, wave field synthesis (WFS) was proposed by Berkhout [J. Audio Eng. Soc., 36, pp. 977-995 (1988)] as a new and powerful concept for spatial reproduction of sound fields without sweet-spotlimitations. Based on this concept, with implementation of simplifications not deteriorating the perceptual quality, a system for variable acoustics was developed: acoustic control system (ACS). The system has been widely applied as a solution for multipurpose halls. Until now, ACS processors have been built using analog electronic technology. Recently, however, a digital module has been designed that not only enables us to generate the ACS reflection patterns, but also to perform true WFS, e.g., for direct sound reinforcement with preservation of natural spatial properties. In the paper, design and specifications of the module will be discussed. Also, the potentials of integrated application of WFS and variable acoustics will be explained. 
2aSP11. Multichannel high sampling rate 1-bit audio with a high definition video recorder system. Jun-ichi Fujimori (Sound and Acoust. Group, Sound Technol. Development Ctr., Yamaha Corp., 203 Matsunokijima, Iwata, Shizuoka, Japan, fujimori-j@beat.yamaha), Yasuhiro Oikawa, and Yoshio Yamasaki (Waseda Univ., Tokyo, Japan)

Ideally, as much audio, video, and metadata information as possible should be acquired to produce archives using the most advanced technology available at the time so that maximum information can be inherited. On the other hand, it is not easy to develop both audio technology and video technology simultaneously or even to integrate them. For example, because we specifically examine audio technology, it is desirable to use video products in the market to achieve the goal. In this sense, we propose the use of IEEE1394 equipment and synchronize their components for integration. We developed a high sampling rate 1-bit audio AD/DA converter that is equipped with IEEE1394 and recording and playback software running on Linux. The software can synchronize a 1-bit audio stream using MPEG-TS of HD video to extend its audio quality using IEEE1394 isochronous streaming with 125-Es resolution. This integration of audio and video is achieved without modifying existing video products. This paper describes the system and the 1-bit audio AD/DA converter $(5.6 \mathrm{MHz}$ sampling $8 \mathrm{ch}$ in $8 \mathrm{ch}$ out or $4.8 \mathrm{MHz}$ sampling 16ch in 16ch out).
2aSP12. Audio noise reduction utilizing inharmonic-frequency analysis of generalized harmonic analysis (GHA). Teruo Muraoka (Res. Ctr. For Adv. Sci. and Technol., The Univ. of Tokyo, 4-6-1, Komaba, Meguro-ku, Tokyo, 153-8904, Japan) and Thoru Ifukube (The Univ. of Tokyo, Tokyo, 153-8904, Japan)

Audio noise reduction is a desired technology for better telecommunications. Currently, the technology is based mostly upon spectral subtraction methods. However, its fundamental problem of musical noise generation remains unsolved. Musical noise is produced by mismatching of instantaneous phases of presampled noise and target noise to be canceled, which causes noise-breathing effects synchronous with the object's intensity variation. Additionally, unnecessary frequency components inherent to Fourier analysis to resynthesis process are mixed in the processed output. For this reason, spectral subtraction is inapplicable to high-fidelity audio. The authors have devoted attention to inharmonic frequency analysis of generalized harmonic analysis (GHA), which was originally proposed by Wiener. The analyses were intended to examine stochastic signals utilizing harmonic frequency analysis. Results clarified that any signal can be represented as an almost periodic function whose frequency components have an inharmonic relationship. In 1993, Dr. Hirata proposed inharmonic frequency analysis based upon successive matching with frequency component templates, which is applicable to high-fidelity audio signal processing. This method came to be called GHA, following Wiener's theory. The authors mostly attempted noise reduction in damaged SP records of historical value, retrieving almost noise-free musical sounds.

\title{
Session 2aUW
}

\section{Underwater Acoustics and Acoustical Oceanography: Sediment Acoustic Processes: Quantifying the Effects of Sediment Properties on Dispersion, Attenuation, Reflection, Scattering, and Buried Target Detection I}

\author{
Eric I. Thorsos, Cochair \\ Univ. of Washington, Applied Physics Lab., 1013 NE 40th St., Seattle, WA 98105-6606 \\ Masao Kimura, Cochair \\ Tokai Univ., Dept. of Geo-Environmental Technology, 3-20-1 Shimizu-Orido, Shizuoka 424-8610, Japan
}

Chair's Introduction-7:50

Invited Papers

2aUW1. The Sediment Acoustics Experiment in 2004 (SAX04). Eric I. Thorsos, Kevin L. Williams, Dajun Tang, Steven G. Kargl Darrell R. Jackson, and Brian T. Hefner (Appl. Phys. Lab., Univ. of Washington, 1013 NE 40th St., Seattle, WA 98105-6698, eit@apl.washington.edu)

A major sediment acoustics experiment (SAX04) was conducted near the coast of northwestern Florida, USA during SeptemberNovember 2004. In addition to our laboratory, a number of other institutions participated, principally from the USA and Canada. SAX04 addressed sound penetration into, propagation within, and scattering from the shallow-water seafloor, as well as scattering from proud and buried targets. The sandy sediment at the experiment site has a critical angle of nearly $30 \mathrm{deg}$, convenient for study of acoustic penetration into sediments at grazing angles below the critical angle. A shallow-water depth of about $17 \mathrm{~m}$ allowed divers to be utilized in many aspects of the experimental work. In particular, divers deployed a 30-m rail system on the bottom for synthetic aperture sonar and other acoustic measurements. SAX99, conducted at a site nearby, showed the importance of scattering from ripples on high-frequency acoustic penetration at subcritical grazing angles. In part because of these findings, a new research initiative on sediment ripple formation, evolution, and decay was begun by ONR, and investigators from this program carried out studies at the SAX04 site. An overview will be given of the SAX04 measurement program. [Work supported by ONR.] 
2aUW2. Observations of seafloor ripples and a time-dependent ripple geometry model. Peter Traykovski (Appl. Ocean Phys. Dept., Woods Hole Oceanogr. Inst., Woods Hole, MA, 02543)

Recent seafloor acoustic scattering experiments and theoretical developments have shown that the presence of ripples on the seafloor can increase the amount of subcritical angle acoustic penetration into the seafloor. A ripple observation and acoustic scattering experiment (SAX04) was recently conducted near Fort Walton Beach, Florida. During this experiment a sidescan sonar on an REMUS AUV was used to observe ripple geometry from the nearshore (5-m depth) to the shelf break (50-m depth). The ripples were found to increase in wavelength from $20 \mathrm{~cm}$ in the nearshore to $1.2 \mathrm{~m}$ at the shelf break. The offshore ripples were a product of Hurricane Ivan, which had passed the site 2 weeks prior. Time series of ripple geometry, measured with a tripod-mounted rotary sidescan sonar and a two-axis rotary pencil beam sonar, from a coarse sand site at the Martha's Vineyard Coastal Observatory, also revealed long-wavelength $(0.6$ to $1.2 \mathrm{~m})$ relict ripples after wave events. Ripple geometry models that assume the ripples are in equilibrium with hydrodynamic forcing underpredict the wavelength of the relict ripples at both sites; thus a new model that allows for a time delay between ripple adjustment and forcing was developed. [Work supported by ONR.]

\section{$8: 35$}

2aUW3. Biodegradation of wave-formed sand ripples during SAX04 (Sediment Acoustics Experiment 2004). Alex E. Hay (Dept. of Oceanogr., Dalhousie Univ., Halifax, NS B3H 4JI, Canada)

The gradual decay of 50- to 70-cm-wavelength sand ripples over a 20-day period of continuous in situ observations is presented. The ripples formed midway through the SAX04 experiment during Tropical Storm Matthew, and the observation period began immediately following this event. Bed elevation profiles and planform images of the ripple field were obtained at centimeter-scale resolution using rotary sonars, and at millimeter-scale resolution using a laser-video bed profiling system. Low-frequency currents, wave orbital velocities, and near-bed turbulence were monitored simultaneously with colocated instruments. The observed rates of change of the principal components of the bed elevation spectrum and of the spatially integrated backscatter intensity differences are presented and compared to predictions made with a diffusivity-based model of the ripple decay process. The model-data comparisons yield values for the horizontal sediment diffusivity which are consistent with previous measurements using particle-counting techniques.

8:55

2aUW4. Velocity dispersion in water-saturated granular marine sediments. Masao Kimura (School of Marine Sci. and Technol., Tokai Univ., 3-20-1 Orido, Shimizu-ku, Shizuoka, Shizuoka 424-8610, Japan, mkimura@ scc.u-tokai.ac.jp)

The velocity dispersion in water-saturated granular marine sediments is an important subject that has remained unresolved in the community of sediment acoustics. Reported velocity dispersion results cannot be explained using the Biot model with the constant frame bulk modulus. The author showed that the values of the frame bulk modulus of water-saturated glass beads and beach sands are approximately $10^{9} \mathrm{~Pa}$ at a frequency of $500 \mathrm{kHz}$ and about ten times greater than those used typically in the community. Moreover, the author proposed a modified gap stiffness model incorporated into the Biot model (BIMGS model). Using this model, the author demonstrated that the frame bulk modulus has frequency dependence [M. Kimura, J. Acoust. Soc. Am. 1205 (2006)]. In this study, the BIMGS model is established using the measured results of the longitudinal wave velocities in glass beads saturated with alcohol-water mixed liquids. Then the velocity dispersion in glass beads and beach sands with different grain sizes are analyzed using the measured results of the longitudinal wave velocity at a much higher frequency and in the region of relaxation frequency. Finally, it is shown that the reported velocity dispersion can be explained using the BIMGS model.

\section{Contributed Papers}

\section{9:15}

2aUW5. Sound waves and shear waves in saturated unconsolidated sediments. Michael J. Buckingham (Marine Physical Lab., Scripps Inst. of Oceanogr., UCSD, 9500 Gilman Dr., La Jolla, CA 92093-0238)

A recent, continuum-mechanics theory of compressional and shear wave propagation in saturated unconsolidated sediments relies on grainto-grain interactions as the sole source of internal stress in the porous medium. On the basis of a statistical argument, nonlinear microscopic processes occurring at grain boundaries are incorporated into the intrinsically linear grain-shearing theory. The result is two sets of algebraic dispersion relationships, one of which represents a compressional wave and the other a shear wave. The shear wave is predicted to exist even in the absence of an elastic "frame." For both types of wave, the theory predicts logarithmic dispersion and a near-linear dependence of attenuation on frequency. The grain-shearing dispersion relations, which are functions not only of frequency but also of the geophysical parameters of the sediment (porosity, bulk density, grain size, and overburden pressure), show excellent agreement with multiple data sets that have appeared in the literature over the past half century. Since the physical mechanisms behind the grain-shearing theory and Biot's classical theory of wave propagation in porous media are quite different, some comments will be offered in an attempt to clarify the circumstances in which the respective theoretical approaches might be valid. [Research supported by ONR.]

\section{$9: 30$}

2aUW6. Frame virtual mass for unconsolidated granular media with slippage. Nicholas P. Chotiros and Marcia J. Isakson (Appl. Res. Labs., Univ. of Texas, P.O. Box 8029, Austin, TX 78713-8029)

A model of the grain contact physics in the presence of a propagating wave is extended to included losses due to slippage. Mechanical coupling between grains in a randomly packed unconsolidated granular medium is inherently stochastic. Grain-to-grain contact asymmetry leads to coupling of kinetic energy into orthogonal linear and rotational motion at the grain level, and partitioning of the energy. The result is an increase in inertia, which may be treated as an additional or virtual mass term for both shear and compressional waves. The virtual mass term can be evaluated, given the scintillation index of the compressional and shear contact stiffness, Poisson's ratio, and the average number of contact points at each grain. The effect of slippage at the grain-to-grain contact is examined. [Work supported by Office of Naval Research, Ocean Acoustics.] 
dicted by pseudospectral FDTD simulations that used colocated vibracore data as input. Robustness of the attenuation and dispersion measurements

2aUW7. A comparison of two observations of compressional wave velocity dispersion in marine sediments. Charles W. Holland (Appl. Res. Lab., The Penn State Univ., State College, PA), Altan Turgut (Naval Res. Lab., Washington, DC), Jan Dettmer, and Stan Dosso (Univ. of Victoria, Victoria, BC, Canada)

Compressional velocity dispersion exists in all marine sediments. If the dispersion is great enough, it may play a significant role in acoustic interaction with the seabed. On the other hand, if dispersion is weak, seabed models and databases can be substantially simpler. The ocean acoustics community is divided on this issue, in part because of the lack of observations. Here we compare two recent methods for measuring dispersion. One technique measures the broadband reflection coefficient (frequency dependence of the critical angle) and thus infers the velocity dispersion. The other technique measures time of flight in an in situ probe over a broadband of frequencies. Measurements on New Jersey Shelf (STRATAFORM area) are compared and implications for the frequency dependence of the compressional wave attenuation are discussed. [Work supported by the Office of Naval Research and NATO Undersea Research Centre.]

\section{0:00-10:15 Break}

\section{0:15}

2aUW8. Low-frequency laboratory measurements of sound speed in water-saturated granular sediments. Theodore F. ArgoIV (Appl. Res. Labs., Univ. of Texas, P.O. Box 8029, Austin, TX 78713-8029) and Preston S. Wilson (Univ. of Texas, Austin, TX 78712-0292)

Accurate knowledge of the acoustic behavior of water-saturated granular sediments is required for effective sonar operation in certain environments. Acoustic dispersion at frequencies below about $20 \mathrm{kHz}$ has been the topic of much recent study and debate. While the understanding of real ocean-bottom sediments in their full complexity is the ultimate goal, laboratory measurements are significantly less difficult and subject to less uncertainty. This plays an important role in sediment acoustics since the difference between the predictions of competing propagation models is only a few percent. A new laboratory impedance tube apparatus, which operates in the $0.5-10-\mathrm{kHz}$ frequency range, has been developed to address this issue. The device can be operated as a traditional acoustical impedance tube, in which water-borne sound waves interact with the water-saturated sediment. The device can also be operated as a drivingpoint impedance instrument, in which the mechanical input impedance of a column of water-saturated sediment is investigated. The effective-fluid sound speed is then inferred from the impedance measurements. Preliminary measurements of the sound speed in reconstituted natural sand sediments and artificial granular sediments obtained with the new apparatus will be presented and compared to existing theory. [Work supported by ONR.]

\section{0:30}

2aUW9. In situ acoustic scattering, attenuation, and dispersion measurements in marine sediments. Altan Turgut, Roger Gauss (Naval Res. Lab., Washington, DC 20375), Charles Holand (The Penn State Univ., State College, PA 16804), and Lisa Zurk (Portland State Univ., Portland, OR 97207)

The degree of acoustic velocity dispersion and nonlinear frequency dependency of attenuation in various types of sediments is investigated. A down-looking chirp sonar and a wideband acoustic probe system are used to measure compressional wave speed and attenuation within $2-150-\mathrm{kHz}$ frequency band. Measurements indicate slight velocity dispersion and nonlinear frequency dependency of attenuation in silty and sandy sediments that can be effectively modeled by an extended Biot theory (Yamamoto and Turgut, J. Acoust. Soc. Am. 83, 1744-1751 (1988)]. The degree of acoustic scattering due to volume inhomogeneities and surface roughness is also estimated from the incoherent components of the measured signals. Acoustic scattering results are favorably compared with those preby using chirp sonar and acoustic probe systems is also verified by the FDTD simulations [Work supported by the ONR.]

\section{0:45}

2aUW10. Weak consolidation model for wave propagation in ocean sediments. James Berryman (Lawrence Berkeley Natl. Lab., 1 Cyclotron Rd., Berkeley, CA 94720)

Within the context of Biot's theory of poroelasticity, both effective density approximations and grain-to-grain elastic and inelastic effects are incoporated into a complete theory of acoustic propagation through oceanbottom sands. The sedimentary material is assumed to be at best weakly consolidated. A variety of possible attenuation mechanisms are considered in order to explain ultrasonic data for both laboratory studies on glass beads saturated with water and silicon oil, as well as in situ data from the SAX99 experiment. An effective medium model is introduced to treat the weakly consolidated frame constants. Wave speeds and attenuations are explained to first order by the effective density approximation alone, but the remaining small discrepancies in velocity can be modeled successfully using the consolidation constants. Remaining attenuation discrepancies can then be fit using several methods including (a) increase of viscosity in fluid trapped between grains, (b) increase of viscosity due to high frequencies alone, and also (c) a new approach that specifically takes dynamicbut small amplitude-grain rearrangements into account. None of these mechanisms was taken into account in Biot's original low-frequency theory.

\section{1:00}

2aUW11. Frequency dependences of absorption and scattering components of attenuation for water-saturated sands. Keiichi Ohkawa (Tech. R\&D Inst., JDA, 5-2 Ichigayahonmuracho Shinjuku-ku, Tokyo, Japan)

Frequency dependences of attenuation of sound waves in watersaturated marine sands are discussed. Measured attenuation includes a scattering component, and this makes it difficult to understand the frequency dependences of attenuation. By removing the scattering component from the measured attenuation, the Kramers-Kronig (K-K) relationship gives the correct sound speed dispersion. Consequently, the intrinsic attenuation extracted from the data collected during SAX99 experiments follows the Biot theory for overall frequencies. This suggests that the attenuation coefficient of sound waves for water-saturated sands is not linear in $f$. The sound speeds recalculated via the $\mathrm{K}-\mathrm{K}$ relationship are also in excellent agreement with the Biot theory at high frequencies.

\section{1:15}

2aUW12. Dispersion and attenuation due to scattering from heterogeneities in the porosity of sand sediments. Brian T. Hefner, Darrell R. Jackson (Appl. Phys. Lab., Univ. of Washington, 1013 NE 40th St., Seattle, WA 98105-6698), Joseph Calantoni, and Allen H. Reed (Naval Res. Lab., Stennis Space Ctr., MS 39529)

Close packed granular materials are inherently heterogeneous at small length scales due to the random packing of the grains. These heterogenieties should manifest themselves in the frame moduli and the porosity of the medium. For sound propagation through fluid-saturated granular materials, the small scale of the heterogenieties may lead to scattering from the fast compressional wave into the slow compressional wave, increasing the attenuation of sound in the medium. This mechanism may account for the increase in high-frequency attenuation relative to expectations based upon Biot theory observed in recent measurements in sand and glass-bead sediments. Previously, we presented the results of a perturbation theory of sound propagation that accounted for losses due to scattering from heterogenieties in the bulk frame modulus [B. T. Hefner et al., J. Acoust. Soc. Am. 119, 3447 (2006)] and showed that the losses due to this mechanism were not enough to account for the measured attenuations. The theory has since been expanded to account for heterogenieties in the porosity. This 
theory relies on knowledge of the spatial statistics of the porosity and efforts are underway to measure these spatial variations in both simulations of glass beads and through CT scans of sand sediments. [Work supported by ONR.]

\section{1:30}

2aUW13. Measurements of the frequency dependence of sound speed and attenuation in a sandy sediment. Brian T. Hefner, Darrell R. Jackson, Kevin L. Williams, and Eric I. Thorsos (Appl. Phys. Lab., Univ. of Washington, 1013 NE 40th St., Seattle, WA 98105-6698)

Among the systems deployed during the sediment acoustics experiments, SAX99 and SAX04, were a fixed buried hydrophone array and a diver-deployed mobile array. The buried hydrophone array was used to measure the sound penetration into the sediment from a mobile source tower over a frequency range of $11-50 \mathrm{kHz}$ during SAX99. For very steep incident grazing angles well above the critical angle, the penetrating field is only weakly influenced by the surface roughness and can be used to determine the sound speed and attenuation in the sediment. For SAX04, the frequency range was extended such that the new range covered 2-50 $\mathrm{kHz}$. The diver-deployed array consisted of two sources and two receivers, which could be inserted into the sediment to a depth of $10 \mathrm{~cm}$ to measure sound speed and attenuation from $100-260 \mathrm{kHz}$ during SAX99. For SAX04, the frequency range was increased to $40-260 \mathrm{kHz}$ such that the measurements overlapped those made using the buried array. The sound speeds measured during both experiments using these systems are consistent with the predictions of Biot theory while the attenuation follows a linear frequency dependence at high frequencies. [Work supported by ONR.]

\section{1:45}

2aUW14. Frequency dependence of sound speed and attenuation in marine sediments. William M. Carey (Boston Univ., Boston, MA 02215), Ji-Xun Zhou (Georgia Inst. of Technol., Atlanta, GA 30332), and Allan D. Pierce (Boston Univ., Boston, MA 02215)

Zhou and Zhang [J. Acoust. Soc. Am. (2005) (2006)] have reviewed diverse data for propagation in sandy sediments. Attenuation for frequencies below $1 \mathrm{kHz}$ corresponds roughly to $\alpha=0.34\left(f / f_{o}\right)^{2} \mathrm{~dB} / \mathrm{m}$ with the reference frequency being $1 \mathrm{kHz}$. The best-fit exponent is slightly less than 2. Their review of results for frequencies above $10 \mathrm{kHz}$ suggests a substantially different frequency dependence. They considered two explicit models, the Biot model as parametrized and extended by Stoll and the BICSQS model of Chotiros and Isakson [J. Acoust. Soc. Am. (2004)]. In either case the fit of parameters to match sound speed dispersion resulted in an unsatisfactory fit of attenuation versus frequency, and conversely. The present paper reexamines this and contends that, while Biot's formulation is intrinsically correct at low frequencies, Stoll's parametrization is noncausal: Chotiros' model more nearly captures the correct physics in that it is intrinsically causal and includes relaxation processes. A considered extension is to include a continuous smear of relaxation processes (rather than just two) within the basic context of the original Biot model. This is shown to be wholly consistent with Kramers-Kronig relations and to have sufficient flexibility to fit the overall data set.

\title{
Session 2pAA
}

\section{Architectural Acoustics, Speech Communication, Musical Acoustics, Psychological and Physiological Acoustics, and Signal Processing in Acoustics: Special Session in Honor of Manfred Schroeder}

\author{
Ning Xiang, Cochair \\ Rensselaer Polytechnic Inst., 110 8th St., Greene Bldg., Troy, NY 12180 \\ Juergen Schroeter, Cochair \\ AT\&T Labs., Research, 180 Park Ave., Bldg. 103 D163, Florham Park, NJ 07932-0971 \\ Akira Omoto, Cochair \\ Kyushu Univ., Dept. of Acoustic Design, Shiobaru 4-9-1, Minami, Fukuoka 811-8540, Japan \\ Chair's Introduction-1:00 \\ Invited Papers
}

1:05

2pAA1. Manfred R. Schroeder's life in acoustics: Phases, reflections, and other contributions of a "perfect number." Birger Kollmeier (Medizinische Physik, Universitaet Oldenburg, D-26111 Oldenburg, Germany)

Number theory such as the rules to compose a perfect number (which is the sum of all its divisors, e.g., 6, 28, 496, 8128) constitutes only a small part of the many fields of research Manfred R. Schroeder mastered in his scientific life. After finishing his Ph.D. with Erwin Meyer in Goettingen in 1954, Schroeder joined Bell Labs where he eventually became director of acoustics and mechanics research. In 1969 he was also appointed professor of physics and director of the Drittes Physikalisches Institut in Goettingen. He contributed to many seminal publications and inventions in the areas of room acoustics, speech coding, physiological and psychological acoustics, and computer graphics. The Schroeder phases describe the quadratic initial phase relation of the harmonics in a tone complex to produce a maximum flat (speech) waveform. The Schroeder diffusors are reflection gratings based on his number theoretic considerations that suppress the specular reflections while distributing scattered sound into all different directions. The current talk will give an overview of Manfred Schroeder's life in acoustics and his various contributions, including some stories about his unique character. A special focus will be placed on the Schroeder phases and their current use in hearing research. 
2pAA2. Modification of time and frequency dimensions of speech. James L. Flanagan (Rutgers Univ., 26 Wolf Hill Dr., Warren, NJ 07059)

Vocal and auditory mechanisms impose constraints on speech signals. These constraints are central to techniques for coding and bandwidth compression, and hence to efficient transmission and storage. A key representation is the short-time Fourier transform, describing the signal in terms of two variables-time and frequency. This separation affords opportunity, within limits, to scale these information-descriptive parameters. This report recollects efforts (incentivized by bandwidth conservation) to modify speech in time and frequency. The "Analytic Rooter" [Schroeder et al., Proc. IEEE 55, 396-401 (1967)] divides bandwidth by a factor of 2, keeping temporal characateristics intact. The "Phase Vocoder" [J. Flanagan and R. Golden, Bell Syst. Tech. J. 45, 1493-1509 (1966)] allows nonrational manipulations in both time and frequency. And, "Silence Detection," based upon automatic step-size adjustment in adaptive differential PCM coding [Flanagan et al., IEEE Trans. Commun. COM-30, 336-345 (1982)], permits speed variations with constant bandwidth. Recordings illustrate the effects.

\section{$1: 45$}

2pAA3. Early linear predictive speech coding at Bell Telephone Laboratories and Electrical Communication Laboratory. Fumitada Itakura (Meijo Univ., Shiogamaguchi 1-501, Tempaku-ku, Nagoya 468-8502, Japan)

At the 6th ICA in Tokyo (1968), two papers on the linear predictive coding of speech were presented by Manfred Schroeder and by me in the same session. The first paper was on the adaptive linear predictive waveform coding for medium band coding (time domain modeling) and the latter was on the LPC vocoder based on the autoregressive spectral estimation for narrow-band coding (frequency domain modeling). Although their approaches were very different in both concept and scope, there were dramatic common points: the covariance and autocorrelation methods were used to solve normal equation to determine the LPC parameters and also the pitch period was extracted to reproduce speech periodicity in both systems. Subsequently the multipulse (1982), stochastic (1984), and code (1985) excitation LP were proposed at AT\&T BTL, whereas the transformation of LPC parameters to PARCOR (1969) and LSP (1975) and their efficient quantization were intensively studied at ECL of NTT. These efforts were eventually combined for practical implementations in the secure, cellular, and IP telephone in the 1980s. The brilliant ideas by Manfred Schroeder and his associates at BTL, in particular Bishnu Atal, have always been a helpful lighthouse to navigate the world-wide speech coding research in the right direction since 1980s.

2pAA4. Personal reflections on 20 years of research with Manfred R. Schroeder: From concert hall acoustics to code-excited linear prediction. Bishnu S. Atal (Dept. of Elec. Eng., Univ. of Washington, Seattle, WA 98185)

I joined Bell Laboratories, Murray Hill in 1961. Bell Labs then had an excellent environment for challenging researchers to find creative solutions to difficult problems in telecommunication. Coming from India, I was fortunate to have Manfred as my mentor. He and I collaborated on many interesting problems. Stereo sound systems in those days kept the sound confined between two speakers, and we wondered why we could not spread the sound beyond the speakers. We invented a system for creating signals at the human ears that removed this limitation, producing the beginning of surround sound. Vocoders could not synthesize natural-sounding speech, and Manfred always felt that the speech synthesis models used in vocoders were too rigid and inflexible. This kind of thinking led us to the path of adaptive predictive coding and we presented a paper on this topic at the first IEEE meeting on speech communication in Boston in 1967. We continued working together producing better and better speech quality on predictive coders. Our research led to the approach of code-excited linear prediction, which forms the basis of world-wide digital cellular standards.

2:25

2pAA5. The roles of models in speech communication research. Hiroya Fujisaki (Univ. of Tokyo, Tokyo, Japan)

The use of models is a key to development of science and technology. Only through modeling can one test various ideas, hypotheses, and theories against what one observes in nature. This paper first gives a systematic review of models that have been developed in the past on various aspects of speech communication, both between humans and between human and machine. These include speech production models, speech acoustics models, speech perception models, as well as language models and dialogue models. It will then describe briefly some of the models developed by the current author. Finally, it will discuss those aspects that have not been modeled so far, and present the author's prospects on how to model them. This paper is dedicated to Manfred Schroeder, whose distinguished contributions in many areas of acoustics were enlightening to a large number of fellow researchers.

\section{2:45}

2pAA6. Progress in electroacoustic transducer research. Gerhard M. Sessler (Darmstadt Univ. of Technol., Merckstrasse 25, 64283 Darmstadt, Germany, g.sessler@nt.tu-darmstadt.de)

Manfred Schroeder always had a vivid interest in the field of electroacoustic transducers. During his time as Director of Acoustics Research at Bell Laboratories, work on electret transducers was started by Jim West and the author in 1962 and the activities were extended over the years to electret materials, directional microphones, and microphone signal processing. Schroeder himself made some interesting contributions to this field, such as the design of directional microphones based on gradients of various orders with toroidal and unidirectional directivity patterns. Some of these devices were implemented with electret transducers. Work on directional microphones at Bell Labs continued as the author went to the Darmstadt University of Technology. There, studies on silicon transducers were initiated, with the first silicon condenser microphone described in 1983. Recently, a completely new and promising variety of piezoelectric transducers was implemented, consisting of charged cellular polymers. This is a new modification of the electret transducer principle developed in the 1960s at Bell Laboratories. 
2pAA7. Application of digital signal processing to architectural acoustics in the 1960s. James E. West (Johns Hopkins Univ., 3400 N. Charles St., Baltimore, MD 21218)

Digital signal processing techniques in architectural acoustics were first used to investigate the acoustical properties of Philharmonic Hall at Lincoln Center for the Performing Arts in New York City in the early 1960s. The management of Lincoln Center invited the Acoustics Research Department of Bell Labs, headed by Manfred Schroeder, to assist in an evaluation of the Hall because conventional measurement methods were unable to explain its subjective performance. Computer-generated specially shaped tone bursts were played and the sound pressures at different locations in the Hall were recorded and later converted into digital form for evaluation. Special filters were programmed to evaluate reverberation times based on different portions of the decay, and energy distribution in time, space, and frequency domains. Better insights into the relationships between subjective and physical measurements were made possible utilizing the power in digital signal processing.

2pAA8. New directions in room acoustics: Probabilities, computer simulation, number theory. Heinrich Kuttruff (RWTH Aachen Univ., D52066 Aachen, Germany, hku@akustik.rwth-aachen.de)

This paper highlights just a few of M. R. Schroeder's major contributions to room acoustics. In his first investigations Schroeder presented a new approach to the frequency response of rooms by recognizing its random character, at least in the range above a critical frequency limit (today known as the Schroeder frequency). Soon afterwards he began employing the then-new digital computer as a research tool. Together with B. S. Atal he simulated the sound transmission in rooms, and in 1961 both authors demonstrated what nowadays is known as auralization and cancellation of acoustical cross-talk between two loudspeakers. In 1967 they published probably the first paper on ray tracing. Furthermore, Schroeder contributed to measuring techniques, for instance by introducing the integrated impulse response method of recording sound decay. Another astounding achievement is Schroeder's invention of pseudorandom surface structures based upon number-theoretical schemes (Schroeder diffusers). Such devices may be used to scatter sound waves into all directions and hence to improve the acoustics of concert halls by creating lateral sound reflections. By his unconventional ideas and numerous innovations Manfred R. Schroeder has changed our understanding of sound transmission in rooms and initiated many new research activities.

4:00

2pAA9. Acoustic contribution to travel comfort in airplanes - insight delivered from room acoustics. Volker Mellert (Inst. f. Phys., Oldenburg Univ., 26111 Oldenburg, Germany)

Travel comfort in airplanes is affected by numerous parameters from which the acoustic environment provides a major contribution, as revealed in measurements of various symptoms of passenger and crew in long-haul flights. Tools known from room acoustics are useful to calculate and estimate an optimal acoustic design of the cabin interior, e.g., background noise levels must match the demand for privacy, or an appropriate STI ensures good public address and communication between the passenger and crew member. Many measures assume a statistical description of the acoustic field. But the strong frequency-dependant absorption leads to different Schroeder frequencies $f_{s}$, which indicate the transition from well-separated resonances to many overlapping normal modes. In consideration of the extended noise sources as boundary conditions it is useful to determine with $f_{s}$ the regimes of analytical solutions of the wave field within the cabin from a more costly numerical (FEM/BEM) approach.

\section{4:20}

2pAA10. Are concert halls random number generators? Jean-Dominique Polack (UPMC, CNRS, MCC, LAM, 11 rue de Lourmel, F-75011 Paris, France)

In 1954, Manfred Schroeder proved that sound fields in rooms are statistical in nature. Based on his subsequent work, several models of sound fields were derived, both in the frequency and the time domain, that make it possible to predict most of the properties of sound fields in rooms, including the major room-acoustical indices. One of these models, derived by the author, is based on random number generation, another field to which Manfred Schroeder contributed. This model is presented, and its predictions compared to measurements by A. C. Gade and G. W. Siebein in US concert halls (see Gade's presentation at Sabine Centennial symposium in June 1994). Predictions are also compared with current theories of reverberation. However, the model is not able to predict the modal behavior below the Schroeder frequency yet. The solution lays in the so-called semiclassical approximation, which is briefly presented. The case of rectangular halls, where the semiclassical approach is no longer an approximation, but is exact, illustrates how to reconcile Monte Carlo approaches, ray theory, and mode distribution.

\section{4:40}

2pAA11. Schroeder's phase in psychoacoustics. Armin Kohlrausch (Philips Res. Labs. Eindhoven (WO02), HTC 36, NL-5656AE Eindhoven, The Netherlands, armin.kohlrausch@philips.com)

I want to address Manfred Schroeder's contributions to psychoacoustics with a special emphasis on the period 1980 to 1990, when I worked with him at the Third Physical Institute in Goettingen. Among the psychoacoustic topics Schroeder worked on were binaural hearing, often in relation to room acoustics and stereophony, and monaural phase perception. The latter topic was first addressed in an ASA conference abstract from 1959, in which Schroeder emphasized the role of the signal's peak factor for the perceived timbre. This theme was taken up in the famous mathematical paper in 1970, in which Schroeder demonstrated that a quadratic relation between frequency and phase in a (flat-spectrum) harmonic complex allows one to synthesize a periodic signal with a flat temporal envelope 
and a quasilinear frequency modulation. Quite accidentically, it was found out in Bennett Smith's master thesis in 1984 that under specific circumstances, the input phase of the stimulus and the phase characteristic of a fixed point on the basilar membrane can nearly compensate each other. This interpretation, published in 1986 in J. Acoust. Soc. Am. with Schroeder as senior author, initiated a great number of perceptual and physiological studies on the phase characteristics of the inner ear.

5:00

2pAA12. Sequences and their consequences. Michael Vorlaender (Inst. fuer Technische Akustik, RWTH Aachen Univ., D-52056 Aachen, Germany)

Number theory is an inspiration for application in science and engineering. In acoustics, correlation sequences for measurement signals and for diffusors became famous, mainly due to innovative ideas of Professor Manfred Schroeder. [Number Theory in Science and Communication, 3rd ed. (Springer, Berlin, 1999).] Maximum-length sequences were implemented in many real-time analyzers for measuring impulse responses. Their autocorrelation properties are almost ideal and they are widely in use today. Benefits and limitations due to hardware constraints are briefly discussed. Another particular interesting sequence is the Fibonacci sequence. It can be created from a simple loop algorithm as a binary sequence. [H., Schmidt, "Eigenschaften und Realisierung von FibonacciFolgen.," Proc. DAGA88, Braunschweig, p. 621]. The ratio of zeros and ones is converging to the golden ratio, $1.6180 \ldots$. . Its spectrum consists of peaks at frequencies and with amplitudes corresponding to the golden ratio, too. When playing such sequences with certain clock frequency, a strange tonal sound can be heard. In psychoacoustic listening tests the pitch perception and pitch strength were investigated. Moreover, intervals and melodies were played by using a midi interface and new melodic and rhythmic pieces of Fibonacci music were created and checked in listening tests.

WEDNESDAY AFTERNOON, 29 NOVEMBER 2006

KOHALA/KONA ROOM, 1:00 TO 4:55 P.M.

\title{
Session 2pAB
}

\section{Animal Bioacoustics: Fish Bioacoustics: A Tribute to Arthur Myrberg II}

\author{
Richard R. Fay, Cochair \\ Loyola Univ. Chicago, Parmly Hearing Inst., 6525 N. Sheridan Rd., Chicago, IL 60626 \\ Arthur N. Popper, Cochair \\ Univ. of Maryland, Dept. of Biology, College Park, MD 20742 \\ Chair's Introduction-1:00
}

Invited Papers

1:05

2pAB1. Recent discoveries and continuing mysteries in the auditory systems of bony fishes. Peggy L. Edds Walton (Parmly Hearing Inst., Loyola Univ. Chicago, Chicago, IL 60626, peddswa@phi.luc.edu)

Recent research has revealed aspects of the auditory system that reflect the diversity of bony fishes as a group. This presentation will review some of the more exciting finds. For example, the long-standing classification of the hair-cell orientation patterns in saccules may require revision given the diversity of hair-cell orientations documented in closely related fishes recently. Hearing studies have shown that, although auditory specialist fishes (otophysines, with auditory accessories for detection of the pressure component of a sound wave) hear higher frequencies than auditory generalists (those that respond primarily to the particle motion component of a sound wave), work on the toadfish has shown that sensitivity at their best frequencies is not as different as once believed. However, a new kind of auditory specialist has emerged within the herrings. Some herring species respond to ultrasonic frequencies $(i 20 \mathrm{kHz})$, apparently via a specialized region of the utricle. The utricle may be the rising star of the fish auditory periphery as physiological and anatomical-projection studies in nonherring species indicate that the endorgan may serve both the vestibular and the auditory systems. Emerging insights into auditory processing among fishes will be discussed. [Research supported by the NIH.]

$1: 25$

2pAB2. Recent advances in hearing and sound communication by fishes. Friedrich Ladich (Dept. of Neurobiology and Behavior, Univ. of Vienna, 1090 Vienna, Althanstr. 14, Austria)

Acoustic displays have been observed in representatives of 30 or more families of bony fishes. Thanks to Art Myrberg's groundbreaking work, we have insights into the multitude of functions of acoustic signalling in fishes including mate choice, individual and species recognition, reduction of territory intrusions, and interception. Recent studies, many of which have arisen from Art's work, focus on ontogenetic and seasonal changes, female vocalizations, and effects of ambient and anthropogenic noise in hearing and sound communication. In croaking gouramis (labyrinth fishes), for example, ontogenetic development of acoustic communication depends on changes in sound parameters, increases in sound levels, and auditory sensitivities. The importance of seasonal changes in hearing was recently uncovered in toadfish and cyprinids. Generally male fish display acoustically to attract females, but the opposite may 
occur. Only female croaking gouramis vocalize during mating. Ecoacoustical investigations reveal that hearing specialists (cyprinids) are well adapted to the low ambient noise levels of standing freshwaters. They are heavily masked in flowing waters, unlike generalists, which are merely affected by ambient noise. Gaussian and ship noise diminishes a fish's ability to detect sounds and resolve temporal patterns, indicating that human-made noise affects acoustic orientation and communication in fishes. [Work supported by FWF.]

$1: 45$

2pAB3. Seasonal variation in the frequency response of the ear: Implications for communication. Joseph Sisneros (Dept. of Psych., Univ. of Washington, Seattle, WA 98195)

Seasonal variation in vocal-acoustic behavior is well known among nonmammalian vertebrates; however, studies of the neural mechanisms underlying such seasonal variation have focused mainly on the anatomical rather than the neurophysiological correlates of seasonal reproductive periodicity. In this talk, I present the neurophysiological evidence for seasonal variation in the frequency response of the ear in the plainfin midshipman fish (Porichthys notatus). Vocal communication is essential to the reproductive success of the seasonally breeding midshipman fish, which migrates during the late spring and summer from deep ocean sites into the shallow intertidal zone to breed. Female midshipman fish use the auditory sense to detect and locate males that are singing from their nests. The seasonal onset of male mate calling in the midshipman fish coincides with a dramatic increase in the range of frequency sensitivity of the female's saccule, the midshipman's main organ of hearing, thus leading to increased encoding of the male's mate call. I will present evidence for a steroid-dependent mechanism that leads to this form of auditory plasticity and discuss why this plasticity of peripheral auditory frequency sensitivity may represent an adaptation to enhance the acquisition of auditory information needed for mate identification and localization during the breeding season.

2:05

2pAB4. Directional hearing in sharks and other fishes. Anthony Hawkins (Loughine, Kincraig, Blairs, Aberdeen AB12 5YT, UK)

Art Myrberg and his co-workers were among the first biologists to obtain clear evidence that predatory sharks are able to locate sound sources at long distances. Previous experiments had shown that elasmobranches were relatively insensitive to sounds, and it had been suggested that fish were incapable of determining the direction of a sound source in the far field. It was subsequently shown that fish could discriminate between sounds from spatially separated sources in the horizontal and median vertical planes. They could even distinguish between identical sound sources at differing distances. It has been suggested that fish determine direction using particle motion detectors, the otolith organs of the inner ear. An array of vector detectors is capable of detecting the axis of propagation, but as they are inherently bidirectional there remains a problem in detecting the actual direction of the source. This paper considers the proposal that the ambiguities are resolved by phase comparison between the vector detectors and other detectors responding to the sound pressure incident upon the gas-filled swimbladde.

\section{Contributed Papers}

\section{2:25}

2pAB5. Sound production in two loricariid catfishes. Amanda L. Webb and Michael E. Smith (Dept. of Biol., Western Kentucky Univ., Bowling Green, KY 42101)

Many families of catfishes produce sounds via pectoral spine stridulation and/or swim bladder compression using sonic muscles linked to the swimbladder. Sound production capabilities in the catfish family Loricariidae has not yet been examined. We recorded sounds produced by two species of loricariid catfish, Otocinclus affinis and Pterygoplichthys gibbiceps. Both species produced short, broadband clicks via pectoral spine stridulation. Mean fundamental and dominant frequencies for $O$. affinis and P. gibbiceps were approximately 4000 and 8000 , and 600 and 1200 $\mathrm{Hz}$, respectively. Durations of sound pulses were generally shorter in $O$. affinis (2-12 ms) compared to P. gibbiceps (20-100 ms). Scanning electron microscopy was used to examine the dorsal process of the base of the pectoral spines of each species. Sound is produced as the ridges of the pectoral spine base slides past the channel of the pectoral girdle. Distances between dorsal process ridges of $O$. affinis and $P$. gibbiceps were approximately 50 and 160 microns, respectively, with the ratio of this distance to the fish total length being comparable between species. Relationships between spine interridge distance, pulse duration, and spectrum of the sounds produced will be discussed. [We thank John Andersland for SEM assistance.]

2:40

2pAB6. Sound production and hearing ability in the Hawaiian sergeant fish. Karen P. Maruska, Kelly S. Boyle, Laura R. Dewan, and Timothy C. Tricas (Dept. of Zoology, Univ. of Hawaii and Hawaii Inst. of Marine Biol., 46-007 Lilipuna Rd., Kaneohe, HI 96744, maruska@hawaii.edu)

Sounds provide important signals for inter- and intraspecific communication in fishes, but few studies examine behavioral contexts of sound production and hearing ability within a single species. This study characterized the sounds and their behavioral contexts in a wild population of Hawaiian sergeant damselfishes (Abudefduf abdominalis), and compared 
their features to hearing sensitivity measured by both auditory-evoked potentials (AEP) and single-cell recordings in the auditory midbrain. The sergeant fish produces low intensity ( $~ 85-115 \mathrm{~dB}$ rms re: $1 \mu \mathrm{Pa})$, lowfrequency $(\sim 100-400 \mathrm{~Hz})$ sounds during nest preparation, courtship an agonistic interactions. Both AEP and single-cell recordings show that fish are most sensitive to these frequencies $(80-300 \mathrm{~Hz})$ with best frequency at $100 \mathrm{~Hz}$. However, auditory thresholds to tonal stimuli were approximately $20 \mathrm{~dB}$ lower for single-cell recordings than those measured by AEP experiments. Further, midbrain auditory neurons were on average more sensitive to playbacks of conspecific sounds than to pure tones. These experiments show that the hearing ability of this damselfish closely matches the intensity and spectral characteristics of sounds produced in the wild. Multidimensional studies that incorporate analyses of and responses to biologically relevant natural sounds are necessary to understand acoustic processing and the evolution of acoustic communication in fishes.

\section{2:55-3:10 Break}

\section{3:10}

2pAB7. Sound production mechanism in carapid fish: First example with a slow muscle. Eric Parmentier, Jean Paul Lagardere, Pierre Vanderwalle, Jean-Baptiste Braquegnier (Lab de Morphologie Fonctionnnelle et Evolutive, Univ of Liege, Belgium), and Michael L. Fine (Virginia Commonwealth Univ., Richmond, VA 23284-2012)

Fish sonic swimbladder muscles are the fastest muscles in vertebrates and have fibers with numerous biochemical and structural adaptations for speed. Carapid fishes produce sounds with a complex swimbladder mechanism including skeletal components and extrinsic sonic muscle fibers with an exceptional helical myofibrillar structure. To study this system we stimulated the sonic muscles, described their insertion and action, and generated sounds by slowly pulling the sonic muscles. We find the sonic muscles contract slowly, pulling the anterior bladder and thereby stretching a thin fenestra. Sound is generated when the tension trips a release system that causes the fenestra to snap back to its resting position. The sound frequency does not correspond to the calculated resonant frequency of the bladder, and we hypothesize that it is determined by the snapping fenestra interacting with an overlying bony swimbladder plate. To our knowledge this tension release mechanism is unique in animal sound generation.

\section{$3: 25$}

2pAB8. Sound communication by the forceps fish, Forcipiger flavissimus (Chaetodontidae). Kelly S. Boyle and Timothy C. Tricas (Dept. of Zoology, Univ. of Hawaii at Manoa, 2538 McCarthy Mall, Edmondson 152, Honolulu, HI 96822)

The forceps fish, Forcipiger flavissimus (family Chaetodontidae), lacks the anterior swim bladder horns and laterophysic connection that are proposed to improve processing of acoustic signals in the genus Chaetodon. Despite the lack of hearing specializations, laboratory experiments using hydrophones synchronized to video revealed the forceps fish produce at least three sounds: (1) a pulse (21-ms duration, peak frequency $=318 \mathrm{~Hz}$ ) associated with rapid cranial elevation, (2) a low-frequency pulse (9-ms duration, peak frequency $=41 \mathrm{~Hz}$ ) associated with anal fin retraction and elevation, and (3) a high-frequency broadband click (3-ms duration, peak frequency $=7924 \mathrm{~Hz}$ ) associated with tail slap behavior. Auditory evoked potential experiments demonstrate that hearing thresholds for this species are 10-21 dB higher than Chaetodon in the 200-600-Hz band. Highspeed kinematic analyses show that motor movements associated with some acoustic behaviors are different than those in Chaetodon, thus the presence of sound production may be the ancestral state for the family but the mechanisms are divergent. Field observations on Hawaiian reefs reveal that forceps fish occur in pairs and trios that may be haremic, and use acoustic communication among group members.[Work supported by NSF IBN0137883 to TT.]
$3: 40$

2pAB9. Acoustico-lateralis communication in coral reef butterflyfishes. Timothy C. Tricas and Kelly S. Boyle (Dept. of Zoology and Hawaii Inst. of Marine Biol., Univ. of Hawaii at Manoa, Honolulu, HI 96822, tricas@ hawaii.edu)

Butterflyfishes are conspicuous members of coral reefs and well known for their pairing behavior and visual displays during social interactions. Members of the genus Chaetodon have a unique arrangement of the anterior swim bladder horns that connect with the lateral line (the laterophysic connection) and also project towards the inner ear, but functions for this putative acoustico-lateralis adaptation were not known. Field experiments demonstrate that the monogamous multiband butterflyfish, $C$. multicinctus, produces hydrodynamic pulses and sounds during territorial defense, and pulse trains that may be an alert call to the mate. In field choice tests, individuals could distinguish mates from nonmates and produced different calls to each, thus demonstrating the existence of mate recognition and context-specific sound production. Laboratory experiments show that sound production differs among sexes, hydrodynamic pulses are of low frequency $(<100 \mathrm{~Hz})$, and sounds have peak energy from $100-500 \mathrm{~Hz}$. Auditory evoked potential experiments demonstrate that the auditory system has best sensitivity from $200-600 \mathrm{~Hz}$ and that the swim bladder horns provide a $10-20-\mathrm{dB}$ gain within this band. These social sounds produced by Chaetodon can provide relevant stimuli to the auditory, vestibular, lateral line and possibly the laterophysic systems.

\section{$3: 55$}

2pAB10. Ultrasound hearing and response in alosine herrings: A short history of the pure and applied research to date. Carl R. Schilt (LGL Ltd., P.O. Box 416, North Bonneville, WA 98639)

In the early 1990s it was reported that downstream-migrating American shad (Alosa sapidissima) avoided a sonar-sampling beam with fundamental frequency over $150 \mathrm{kHz}$. Since then there has been a considerable body of laboratory and field work to determine which fishes have such hearing capacity, what the hearing limits are, what the hearing mechanisms may be, what might have led to selection for such capacity, and the utility of such stimuli to control the local distributions of fishes with such ultrasonic hearing. So far it seems that ultrasonic click aversion is limited to one subfamily of primarily anadromous herrings (family Clupeidae; subfamily Alosinae). The capacity to hear very high clicks and the propensity to swim away from the stimulus direction has led to experiments in shad protection, especially in hydropower and other industrial environments, which have been promising. This talk will review the history of both the pure and applied science on alosine herring ultrasound response and suggest possible research and application strategies for the future.

\section{4:10}

2pAB11. Continental shelf fish choruses in Australian waters. Robert D. McCauley (CMST Curtin Univ., G.P.O. Box U 1987, Perth 6845 Australia) and Douglas H. Cato (Defence Sci. \& Technol. Org., NSW)

Almost everywhere the authors have sampled ambient noise on Australia's continental shelves, in tropical and temperate waters, evening choruses have been recorded. Chorus maximum frequency varies from a few hundred $\mathrm{Hz}$ to a few $\mathrm{kHz}$ and may result from different species, but the consistency of the timing and similar chorus nature suggests that different source species are following similar behavior. Individual chorus signals are comprised of single pops or clicks of $<200$-ms duration, similar to those described from fish. We hypothesize that these chorus sources are nocturnally active fishes that mainly forage in the water column at night. In the Perth Canyon, Western Australia, shelf break choruses centered at 2 $\mathrm{kHz}$ have been measured since 1992. Here each evening the chorus begins shortly after dusk, reaches a maximum around 2 hours post dusk, drops to a lower level, rises again predawn, and then disappears during the day. Canyon net sampling through the water column has only recovered fish of the family Myctophidae as potential chorus sources. Long sea-noise time series records from the Canyon indicate significant seasonal trends in chorus levels and differences between years, possibly indicating that chorus activity reflects secondary productivity. 
2pAB12. Transmission of air gun array signals in Australian waters. Robert D. McCauley, Susan J. Rennie (Curtin Univ. of Technol., G.P.O. Box U 1987, Perth 6845, Australia), and John R. Hughes (Santos Ltd., Adelaide SA 5000, Australia)

Received air gun array signal parameters from 15 seismic surveys in Australian waters have been analyzed. Measurements include sources from 1115 to 4900 cui $(18.3$ to $80.3 \mathrm{~L}$ ), ranges of hundreds of meters to thousands of kilometers along travel paths of $15 \mathrm{~m}$ to full ocean depth. For any array there may be large $( \pm 9 \mathrm{~dB})$ difference in received level between consecutive signals, especially if two 3-D arrays are operated alternatively. All arrays demonstrate horizontal directivity, being typically omnidirectional below nominally $50 \mathrm{~Hz}$ but focusing higher frequency energy about their longer axis. Three-dimensional arrays tend to be square and 2-D arrays rectangular with the long axis across track; thus 2-D arrays are more focused. At a nominal range, differences of up to $40 \mathrm{~dB}$ were found when comparing small arrays operating in shallow water with large arrays in deeper water. For example, a 2500-cui array transmitting in $70 \mathrm{~m}$ water had no waterborne energy at $20-\mathrm{km}$ range whereas a 4900 -cui array operating on the shelf slope was detectable via a deep sound channel path at $2000-\mathrm{km}$. This large variation in received levels under different scenarios implies similar differences in environmental implications.
2pAB13. Distribution of static pressures on the skin surface of parallely swimming fish. Kazuhiko Anraku, Tatsuro Matsuoka, and Kenji Watanabe (Faculty of Fisheries, Kagoshima Univ., 4-50-20 Shimoarata, Kagoshima 890-0056, Japan, anraku@ fish.kagoshima-u.ac.jp)

Hydrodynamic static pressures on the body surface of fish generated by conspecifics parallely swimming were measured in a flume tank, and they were compared with those generated by changing the position of two individuals under different flow velocities. Two dead common carp (BL $=20 \mathrm{~cm}$ ), fixed in $20 \%$ of formalin, were used. Pressure was measured by a manometer connected to the ends of three aluminum pipes (inner diameter $=3 \mathrm{~mm}$ ), which were inserted perpendicularly to the body axis from right to left in the control fish. The openings of the pipes were located on head, trunk, and caudal regions, and each was on the infraorbital or trunk canal lateral lines. The target fish was moved on the horizontal $X Y$ plane using a traverse device. Although pressures changed depending on the regions measured, changes of pressure according to position and current velocity were relatively low in the caudal region. The absolute values of the pressure increased as flow velocity increased, and decreased with an increase in the distance between individuals. Relative positions when the maximum pressure changes were observed at each flow velocity did not change, indicating that pressures were largest when the maximum body width of the conspecific is closest.

\title{
Acoustical Oceanography and Underwater Acoustics: Acoustic Scattering by Aquatic Organisms IV
}

\author{
Kenneth G. Foote, Cochair \\ Woods Hole Oceanographic Inst., Woods Hole, MA 02543 \\ Masahiko Furusawa, Cochair \\ Tokyo Univ. of Marine Science and Technology, 4-5-7 Konan, Minato, Tokyo 108-8477, Japan
}

Chair's Introduction-1:00

Contributed Papers

1:05

2pA01. Swimbladders under pressure: Anatomical and acoustic responses by walleye pollock. John K. Horne, David Barbee (School of Aquatic and Fishery Sci., Univ. of Washingtons Box 355020, Seattle, WA 98195), Kouchi Sawada, Koki Abe, Kazuhiro Sadayasu, and Yoshimi Takao (Natl. Res. Inst. of Fisheries Eng., Kamisu-city, Ibaraki 314-0408, Japan)

Pressure influences echo intensities of swimbladdered fish through changes in swimbladder volumes and surface areas. Volume reduction is expected to correspond to Boyle's law (volume $\propto$ pressure $^{-1}$ ) but the resulting deformation in swimbladder surface area will largely determine target strength at geometric scattering frequencies. Dorsal and lateral radiographs of juvenile walleye pollock (Theragra chalcogramma) in a pressure chamber were used to image swimbladders from ambient to a maximum of 5-atm pressure. As pressure increased, dorsal swimbladder surface areas decreased at a constant rate among the three individuals. Swimbladder volume reduction rates were similar among individuals but less than that predicted by Boyle's law. Compression of swimbladders occurred dorsal-ventral, anterior-posterior, and laterally. Resulting swimbladder shapes became more spindlelike as pressure increased. KRM predicted target strengths at 38 and $120 \mathrm{kHz}$ decreased more rapidly at $3 \mathrm{~atm}$ above ambient than at lower pressures. [Work supported by ONR and NRIFE.]

\section{$1: 20$}

2pAO2. Modeling of the backscattering by swimbladder-bearing fish. Dezhang Chu, Timothy K. Stanton (Woods Hole Oceanograph. Inst., Woods Hole, MA 02543), J. Michael Jech (NOAA/Northeast Fisheries Sci. Ctr., Woods Hole, MA 02543), and D. Benjamin Reeder (Naval Postgraduate School, Monterey, CA 93943)

A composite scattering model has been developed to describe the backscattering by swimbladder-bearing fish. This model includes the influence of elongation of the swim-bladder not only on the resonance frequency, as do most of the existing scattering models, but also on the quality factor $\mathrm{Q}$ and the overall scattering level. In addition, within the region covering the transition from resonance scattering to geometric scattering, the effect of asymmetric cross section of the swimbladder has also been included to represent a more realistic shape of fish swimbladder. The model was compared with other scattering models, such as Kirchhoff ray mode (KRM) and Fourier matching method (FMM). Model predictions were then compared with field data collected with a new broadband towed system. For a single-species Atlantic herring school (Clupea harengus) with a measured length distribution, the predicted resonance scattering including resonance peak location and overall level matched the broadband acoustic data reasonably well. Some modeling issues will also be discussed. 
1:35

2pAO3. Target strength and behavior of swimming juvenile southern bluefin tuna in a cage estimated by echo-trace analysis. Koki Abe, Yoshimi Takao, Kazuhiro Sadayasu, Kouichi Sawada, and Kazuo Amakasu (Nat. Res. Inst. Fish. Eng., 7620-7 Hasaki, Kamisu, Ibaraki, 314-0408, Japan)

Echo-trace analysis (ETA) was applied to echoes from juvenile southern bluefin tuna (Thunnus maccoyii, SBT) swimming in a cage. The experiments were conducted offshore Esperance in Western Australia, using a split-beam echosounder operating at $70 \mathrm{kHz}$. The dimensions of a cylindrical cage were $7 \mathrm{~m}$ in diameter, $20 \mathrm{~m}$ in depth, and $8.5 \mathrm{~cm}$ mesh size of a net. A transducer was set at the center of a cage. The samples of SBT were caught by trolling near the study site. The age classes were 1 or 2 years old that were ranging 45 to $82 \mathrm{~cm}$ in fork length. Only one SBT was put into a cage at a time in order to obtain single echoes from one fish. As the swimbladder of juvenile SBT was not gas-filled in this stage, its target strength (TS) was much lower than TS of a same-size fish with gas-filled swimbladder. Because of low TS values, there were some difficulties to obtain high quality echo-traces continuously. So, preselections of high signal-to-noise and noninterference echoes from the echogram were needed before applying ETA. Swimming velocities and TS patterns in dorsal aspect were estimated by ETA.

\section{1:50}

2pAO4. An advanced 3-D scattering model for squid. Benjamin A. Jones, Andone C. Lavery, and Timothy K. Stanton (WHOI, Woods Hole, MA 02543-1050)

A three-dimensional, acoustic scattering model for squid has been developed that incorporates high-resolution, spiral computerized tomography (SCT) scans of squid morphology. This model numerically implements the distorted wave Born approximation (DWBA) for an inhomogeneous body using a combined ray trace and volume integration approach. SCT scans of squid, taken in air, revealed detailed morphology of the animal including the interior cavity of the mantle. The model accounts for these features with volume elements that are small (less than $1 / 20$ the wavelength) for the frequencies of interest $(0-120 \mathrm{kHz})$. Target strength predictions are compared with published data from live, freely swimming squid. Although only two materials, mantle tissue and seawater, are used to represent squid in this study, our model can be easily generalized to include many distinct, weakly scattering regions within a volume. Ranges of validity with respect to material properties and numerical considerations are explored using benchmark computations with such simpler geometries as spherical and cylindrical shells.

\section{2:05}

2pAO5. Target strength estimation of the large jellyfish Nemopilema nomurai. Miyuki Hirose (Grad. School of Fisheries Sci., Hokkaido Univ., 3-1-1, Minato Hakodate Hokkaido, 041-8611, Japan), Tohru Mukai, Kohji Iida (Hokkaido Univ., Hakodate Hokkaido, 041-8611, Japan), and Doojin Hwang (Chonnam Nat. Univ., Dundeok-dong, Yeosu Jeollanam-do 550-749, Korea)

The jellyfish Nemopilema nomurai, which reaches up to $2 \mathrm{~m}$ in diameter and $200 \mathrm{~kg}$ in weight, has occurred in large numbers in the Sea of Japan during the last several years and has had a negative effect on coastal fisheries in this region. Data on the abundance and distribution of jellyfish are needed to forecast when and where they will occur in coastal areas. Acoustic techniques are commonly used to study the distribution and abundance of fish and zooplankton because these techniques can survey large areas relatively quickly. However, before such surveys can be conducted, the acoustic characteristics of the target species must be known. In this study, the density of and speed-of-sound in live jellyfish were measured to clarify their acoustic characteristics using a theoretical scattering model. Jellyfish density was measured using the dual-density method, while the speed of sound was measured using the time-of-flight method. The acoustic characteristics were estimated with the distorted-wave Born approximation (DWBA) model using these material properties and the shape of free-swimming jellyfish. The results demonstrate the feasibility of investigating the abundance and distribution of jellyfish using acoustic methods. [Work supported by JSPS and KOSEF Core University Program on Fisheries Science (FiSCUP).]

$$
\text { 2:20 }
$$

2pAO6. Predictions of acoustic scattering by a fish school. Charles F. Gaumond and Ralph N. Baer (Naval Res. Lab., Washington, DC 20375)

A theory originally developed by Richard Love [J. Acoust. Soc. Am. 64, 571-580 (1978)] is used to model scattering by a school of fish. In order to incorporate propagation effects, this model has been integrated into a modified version of the RAM acoustic propagation model developed by Michael Collins. Running the integrated model over a sequence of frequencies results in predictions of the time and frequency response of broadband signals scattered by fish schools. Using an ensemble of fish school realizations lets us develop features that describe the response of a fish school to the acoustic signals. These features in turn allow separation of returns from a target from those from interfering schools. [Work supported by the Office of Naval Research.]

\section{2:35}

2pA07. Using acoustic backscatter to determine the size distribution of channel catfish in a commercial pond. John Heffington, James Chambers (Natl. Ctr. for Physical Acoust., The Univ. of Mississippi, 1 Coliseum Dr., Univ., MS 38677), David Heikes, Steve Pomerleau, and Eric Stone (The Univ. of Arkansas at Pine Bluff, Pine Bluff, AR 71601)

With increasing seafood demand, aquaculture is poised to become a major growth industry in the United States. In particular, channel catfish represents an approximately $\$ 400$ million industry. Pond management strategies, however, are compromised by high fish densities with large size distributions that result from years of continuous culture without a total harvest. Recent work has been presented on the Aquascanner SONAR system, which evaluates the total biomass of the pond but is less effective in determining the size distribution. To address this shortcoming, research colleagues at the University of Arkansas Pine Bluff are using a subsample seine net. Their method involves pulling a trawl across the pond to obtain a sample of the fish and manually weighing the fish in a time-consuming process to provide the size distribution. The work presented here attempts to use acoustic backscatter to determine the size distribution of the fish in lieu of the manual weight measurements. After being seined, the fish are allowed to swim back into the pond through a restrictive pipe and are pinged acoustically to determine their target strength and thus size. The experimental design of the system and results will be discussed. [Work Supported by the USDA.] 


\section{Contributed Poster Papers}

All posters will be on display from 1:00 p.m. to 5:00 p.m. Contributors of all papers will be at their posters from 4:25 p.m. to 5:15 p.m.

2pAO8. Swimbladder morphology and target strength of myctophid fish of the northwestern Pacific. Hiroki Yasuma, Kazushi Miyashita (Field Sci. Ctr. for the Northern Biosphere, Hokkaido Univ., 3-1-1, Minato, Hakodate, Hokkaido, 041-8611 Japan), Kouichi Sawada (Natl. Res. Inst. of Fisheries Eng., Hasaki, Kashima, Ibaraki, 314-0421 Japan), and Ichiro Aoki (Univ. of Tokyo, Bunkyo, Tokyo, Japan)

Swimbladder shapes of fish from 14 myctophid species captured around Japan were measured using an X-ray method to estimate the theoretical target strength. Using those $\mathrm{x}$-ray images, the fish were classified into bladder species and nonbladder species according to the presence of the gas. Five species had no swimbladder in any body size range examined. Others contained bladder and nonbladder fishes in relation to growth. The growth of swimbladder length was not proportional to that of body length (allometric growth) in some bladder fishes. Based on morphological parameters of the body and swimbladder, the relationships between body length and target strength from 38 to $200 \mathrm{kHz}$ were estimated using theoretical acoustical backscattering models. Estimated values of target strength showed greater than 10-dB differences between bladder and nonbladder fishes, even when the fish were of equivalent size. Because of the allometric growth of the swimbladder, values of the target strength were not proportional to the square of the body length in most bladder species. In addition, larger fishes (over $60 \mathrm{~mm}$ ) of Myctophum asperum showed regressive growth of the swimbladder (or target strength), suggesting that the use of the $20 \log$ relationship is inappropriate for bladder species of myctophid.

2pA09. In situ measurement of target strength, tilt angle, and swimming speed of Boreopacific gonate squid (Gonatopsis borealis). Kouichi Sawada, Hideyuki Takahashi, Koki Abe, and Yoshimi Takao (Natl. Res. Inst. of Fisheries Eng., Fisheries Res. Agency, 7620-7 Hasaki, Kamisu, Ibaraki, 314-0408, Japan)

Target strength (TS), mantle length (ML), swimming speed, and tilt angle of Boreopacific gonate squid Gonatopsis borealis were measured using an acoustical-optical composite system (J-QUEST) in the North Pacific Ocean. The J-QUEST consists of a quantitative echosounder operating at $70 \mathrm{kHz}$ and a high-sensitivity stereo video camera system installed in a housing resistant to pressure at the depth of $300 \mathrm{~m}$. In the daytime, Boreopacific gonate squids were seen at the J-QUEST depth of $230 \mathrm{~m}$ by the stereo video camera. Swift vertical movements were also observed on the echogram at the same time. Swimming speeds and tilt angles were estimated by tracking echoes from the same squid. Estimated average swimming speed was over $1 \mathrm{~m} / \mathrm{s}$ and tilt angles were almost vertical. Average TS calculated from squidlike echoes was $-54.1 \mathrm{~dB}$ and average ML calculated from the stereo video images was $23.9 \mathrm{~cm}$ with standard deviation of $5.9 \mathrm{~cm}$. Standardized TS by the squared ML was $-81.9 \mathrm{~dB}$. In the nighttime, a Boreopacific gonate squid (estimated ML $37 \mathrm{~cm}$ ) at the J-QUEST depth of $25 \mathrm{~m}$ was observed preying on one Japanese anchovy, Engraulis japonicus, in an aggregation with estimated average length of $12.3 \mathrm{~cm}$.
2pAO10. Target strength of Sargassum plant with vesicles containing gas. Kenji Minami (Hokkaido Univ., 3-1-1 Minato-cho, Hakodate, Hokkaido, 041-8611, Japan), Akira Hamano, Takeshi Nakamura (Natl. Fisheries Univ., Shimonoseki, Yamaguchi 759-6595, Japan), Yoshimi Takao (Fisheries Res. Agency, Kamisu, Ibaraki, 314-0408, Japan), and Kazushi Miyashita (Hokkaido Univ., Hakodate, Hokkaido, 041-8611, Japan)

Information about the target strength (TS) of a vesicle is important to estimate the acoustic scattering of Sargassum plants. Experimental TS (TSe) of a vesicle was obtained at 70 and $200 \mathrm{kHz}$ using quantitative echo sounders in a freshwater tank $(10 \times 10 \times 15 \mathrm{~m})$. The TSe of ten vesicles examined here were $-56.88-51.68 \mathrm{~dB}$ at $70 \mathrm{kHz}$ and $-60.30--50.90 \mathrm{~dB}$ at $200 \mathrm{kHz}$. The TSe's of small vesicles were $2.56--3.70 \mathrm{~dB}$ higher at 70 $\mathrm{kHz}$ than at $200 \mathrm{kHz}$, suggesting that these small vesicles were affected by the airbladder resonance. Theoretical TS (TSt) was estimated by airbladder-scattering models to confirm this effect. The gas radii of 418 vesicles (containing the experimented ten vesicles) were measured using a soft $\mathrm{x}$-ray system and applied to the theoretical models. Their radii were $0.52--6.00 \mathrm{~mm}$; calculated TSt matched with TSe. According to the theoretical model, the vesicles were not affected by the resonance when argument $k a$ is $k a>1$ (where $k$ is the wave number and $a$ is the radius). However, the vesicles were affected by the resonance when $k a<1$. That fact indicates that small vesicles ( $a$ is $1.22--2.38 \mathrm{~mm}$ ) at $70 \mathrm{kHz}$ were affected by the resonance.

2pAO11. Target strength of a mesopelagic fish Maurolicus japonicus at 38 and 120 kHz. Tadanori Fujino, Hiroki Yasuma, Kazushi Miyashita (Hokkaido Univ., 3-1-1, Minato-cho, Hakodate, Hokkaido, 041-8611, Japan, fnori@fish.hokudai.ac.jp), Kazuhiro Sadayasu, and Koki Abe (Fisheries Res. Agency, Hasaki, Kamisu, Ibaraki, 314-0408, Japan)

Target strength (TS) of Maurolicus japonicus was examined at 38 and $120 \mathrm{kHz}$ using experimental measurements (at $38 \mathrm{kHz}$ ) and theoretical swimbladder scattering models. Experimental measurements showed that the average TS for dominant size ( 32.1 to $34.8 \mathrm{~mm}$ standard length) were -60.5 to $-56.7 \mathrm{~dB}$, and the effect of the fish orientation on TS was slight. These results coincided with the model estimation, proving that swimbladder models are valid to estimate the TS of $M$. japonicus. The slight effect of the fish orientation on TS was presumed due to the oval shaped swimbladder with high aspect ratio, which is relatively little to the wavelength. This is a merit because tilt angle is usually a major source of TS variation. However, a little swimbladder would cause acoustic resonance at low frequencies, which leads to insatiability on TS according to depth change. Using the resonant model, relationships of the TS and standard length were estimated for depth 50,100,150,200, and $250 \mathrm{~m}$ at 38 and $120 \mathrm{kHz}$, respectively. While TS at $120 \mathrm{kHz}$ showed little change according to depth, TS at $38 \mathrm{kHz}$ changed with depth and showed larger TS than 120 $\mathrm{kHz}$ for each size. 
2pA012. Target strength pattern measurement of krill (Euphausia pacifica) and measuring limits in a small tank. Kouichi Sawada (Natl. Res. Inst. of Fisheries Eng., Fisheries Res. Agency, 7620-7, Hasaki, Kamisu, Ibaraki, 314-0408, Japan), Tohru Mukai (Hokkaido Univ., Hakodate, 041-8611, Japan), Kazuo Amakasu, Koki Abe, and Ken Ishii (Fisheries Res. Agency, Kamisu, Ibaraki, 314-0408, Japan)

Euphausia pacifica is an extremely important species for Japanfs northeast coastal fisheries and a key species for ecosystems in the northwest Pacific Ocean. Target strength (TS) of krill information have been required for acoustic surveys; they constitute a scale factor that is used to convert acoustic estimates of abundance. A precise TS pattern measurement system was constructed in a small tank. Target strength patterns of a target are measurable in the angle range of 60 deg at 1-deg steps. First, TS values of the thinnest fishing line with 53-mm diameter were measured as it was planned for use in tethering krill. TS measurement limits were also confirmed by measuring echo signals and noise signals. Maximum TS of the fishing line was $-95 \mathrm{~dB}$ and the measurement limit was $-98 \mathrm{~dB}$ at the frequency of $200 \mathrm{kHz}$ with $10 \mathrm{~dB}$ of signal-to-noise ratio (SNR). Second, we confirmed the validity of the target suspension method. The thinnest fishing line was penetrated into the krill body to tether it between two vertical fishing lines. Finally, TS patterns of 12 live krill were measured and compared with theoretical calculations using distorted wave Born approximation. Both agreed well.

\section{3:10-3:25 Break}

\section{Contributed Papers}

3:25

2pA013. Estimation of swimming behavior and backscattering pattern of Antarctic krill using echo-trace-analysis. Kazuo Amakasu (Natl. Res. Inst. of Fisheries Eng., Fisheries Res. Agency, 7620-7 Hasaki, Kamisu, Ibaraki, 314-0408, Japan, soundproc@yahoo.co.jp), Masahiko Furusawa (Tokyo Univ. of Marine Sci. and Technol., Minato-ku, Tokyo, 108-8477, Japan), and Sakiko Ando (Ocean High Technol. Inst. Inc., Nakano-ku, Tokyo, 164-0012, Japan)

Echo-trace-analysis was applied to single echo-traces of Antarctic krill to estimate in situ swimming orientation, swimming speed, and backscattering pattern. Acoustic data were collected with a split-beam echosounder operating at $70 \mathrm{kHz}$ on 11 January 2005 during the Southern Ocean survey by the training vessel Umitaka-maru of Tokyo University of Marine Science and Technology. While the acoustic data were collecting, the ship speed was 2 knots. A pulsewidth and a pulse-repetition-period of the echosounder were $0.6 \mathrm{~ms}$ and $0.27 \mathrm{~s}$, respectively. Krill aggregations were observed at the depth range $15-30 \mathrm{~m}$ from the hull-mounted transducer and at upper and lower edges of them some single echo-traces were visually recognized on echograms. In this work, these echo traces were analyzed. As preliminary results, the average swimming orientation was 4.0 deg with a standard deviation of $14.2 \mathrm{deg}$ and the average swimming speed was $0.33 \mathrm{~m} / \mathrm{s}$ with a standard deviation of $0.16 \mathrm{~m} / \mathrm{s}$. The average swimming orientation was close to the orientation observed with a video plankton recorder by Lawson et al. [J. Acoust. Soc. Am. 119(1), 232-242 (2006)]. The estimated backscattering patterns showed peaks at incident angles around -10 to $10 \mathrm{deg}$ and were similar to the patterns predicted by theoretical models.

\section{$3: 40$}

2pA014. Development and application of multifrequency acoustic techniques to the study of Antarctic krill distribution. Gareth L. Lawson, Peter H. Wiebe, Timothy K. Stanton, and Carin J. Ashjian (Woods Hole Oceanog. Inst., Woods Hole, MA 02543)

Multifrequency acoustic techniques were refined and applied in a study of the distribution of Antarctic krill. Acoustic, video, and net data were collected in a continental shelf region west of the Antarctic Peninsula in the falls and winters of 2001 and 2002. Improved parametrization of a theoretical model of krill target strength was achieved through direct measurement of all model parameters. Krill visual acuity was used to determine a threshold level of volume backscattering strength for identifying krill aggregations. Differences in mean volume backscattering strength at 120 and $43 \mathrm{kHz}$ further served to distinguish krill from other sources of scattering. Inversions were performed of mean volume backscattering strength $(43,120,200$, and $420 \mathrm{kHz})$ in these acoustically identified ag- gregations to estimate the weighted mean length and density of krill. Inversion results were comparable to net samples for estimated length, but acoustic estimates of krill density exceeded those from nets by one to two orders of magnitude, likely due to avoidance and differences in the volumes sampled by the two systems. Application of these methods to survey data demonstrated strong spatial and temporal variability in the horizontal and vertical distribution of krill aggregations, consistently associated with food availability, temperature, and predators.

\section{$3: 55$}

2pA015. Acoustic studies investigating the distribution and abundance of krill in critical blue whale habitat in the Monterey Bay submarine canyon. Louise P. McGarry, Charles H. Greene (Cornell Univ., Snee Hall, Ithaca, NY 14853), Peter H. Wiebe (Woods Hole Oceanog. Inst., Woods Hole, MA 02543), and Donald A. Croll (Univ. of California, 100 Shaffer Rd., Santa Cruz, CA 95060)

Blue whales, the largest animal on the planet, are frequently found in concentrations at the ocean surface in areas over thick sound-scattering layers of krill. In this study, the dive behavior of a blue whale was investigated in the context of zooplankton acoustic backscatter data concurrently collected in the Monterey Bay submarine canyon. The whale dive behavior revealed that the whale spent greater than 50 percent of its time in the region of the most intense backscatter, suggesting threshold foraging behavior. In order to describe the prey field within the canyon, additional day and night acoustic surveys with MOCNESS net tows in the canyon and along its perimeter were collected. Here, a three-dimensional characterization of the prey field is developed using the acoustic data. Further work will be undertaken to estimate the relative biomass of the zooplankton taxa generating the acoustic backscatter within and without the intense sound-scattering layer, and to model the acoustic backscatter that would be expected to be generated from the zooplankton specimens collected: the forward problem. [This work is supported by the ONR.]

\section{$4: 10$}

2pA016. Multiple-angle acoustic scattering and classification of zooplankton. Paul L. D. Roberts and Jules S. Jaffe (Marine Physical Lab, Scripps Inst. of Oceanogr., La Jolla, CA 92093-0238)

The use of multiple-angle acoustic scattering to discriminate between two ecologically important classes of zooplankton' copepods and euphausiids, is proposed. The distorted wave Born approximation is used to model the multiple-angle scatter from a linear array of transducers designed to be practical for field deployment. Using a large set of noisy training and test data generated from uniformly random length and threedimensional orientation distributions from each class of scatterers, the 
performance of classification algorithms in several feature spaces is evaluated. The results show a marked improvement in classification performance as additional angles, and larger angular separations, are included in the classifier. Interestingly, even in the case of uniformly random three- dimensional scatterer orientation, low classification error $(\sim 5 \%)$ can be obtained. These results hold promise for substantially improving the classification of fluidlike zooplankton in situ using multiple angle scatter obtained with a simple collection geometry.

WEDNESDAY AFTERNOON, 29 NOVEMBER 2006

KAHUKU ROOM, 1:00 TO 3:15 P.M.

\title{
Session 2pBBa
}

\section{Biomedical Ultrasound/Bioresponse to Vibration: Topical Meeting on Shock Wave Therapy II}

\author{
S. H. R. Hosseni, Cochair \\ Tohoku Univ., Inst. of Fluid Science, 2-1-1 Katahira, Aoba, Sendai 980-8577, Japan \\ Robin O. Cleveland, Cochair \\ Boston Univ., Aerospace and Mechanical Engineering, 110 Cummington St., Boston, MA 02215
}

\section{Contributed Papers}

\section{1:00}

2pBBa1. Radiation force imparted on a kidney stone by a Dopplermode diagnostic pulse. Oleg A. Sapozhnikov (Ctr. for Industrial and Medical Ultrasound, Appl. Phys. Lab., Univ. of Washington, 1013 NE 40th St., Seattle, WA, 98105), Leonid A. Trusov (Moscow State Univ., Moscow 119992, Russia), Alexander I. Gromov (Mandryka 2nd Central Clinical Military Hospital, Moscow 107014, Russia), Neil R. Owen, Michael R. Bailey, and Lawrence A. Crum (Univ. of Washington, Seattle, WA, 98105)

Detection of kidney stones and estimation of their sizes is an important part of the lithotripsy treatment. Fluoroscopy is often used to target stones, but not every stone is radio-opaque and, in addition, fluoroscopy produces ionizing radiation. Acoustic waves offer an alternative way to visualize stones. The acoustic impedance of kidney stones typically differs significantly from that of surrounding tissue. A useful consequence of the impedance mismatch is the possibility to target stones with diagnostic mode ultrasound. Another consequence is that radiation force pushes the stone. Stone displacement may be responsible for the twinkling artifact that has been observed by several authors in color Doppler mode of ultrasound imaging. This effect can be used to detect not only renal and ureteral stones, but also calcifications in other organs (e.g., breast). In this paper we model the radiation force associated with the Doppler diagnostic pulse. The problem is divided into three parts: (1) acoustic scattering; it is solved in finite differences; (2) radiation force calculation; (3) stone velocity estimation supposing the stone sits in soft tissue. [Work supported by NIH DK43881, DK55674, NSBRI SMS00402, and RFBR.]

\section{$1: 15$}

\section{2pBBa2. Numerical solutions of the} Khokhlov-Zabolotskaya-Kuznetsov (KZK) equation satisfying the Rankine-Hugoniot condition. Gianmarco Pinton and Gregg Trahey (Duke Univ., 136 Hudson Hall, Box 90281, Durham, NC 27708, gfp@duke.edu)

A numerical method that solves the KZK equation and satisfies the Rankine-Hugoniot relation for shock-wave propagation is described and characterized. By comparison with a known planar solution, it is demonstrated that current numerical methods in both the time and frequency domains predict a shock front that is stationary relative to the propagation phase and that the proposed method predicts the correct speed. These methods are then compared in the context of shock-wave lithotripsy and high intensity focused ultrasound. At the focus, axisymmetric shock-wave lithotripter simulations show that the Rankine-Hugoniot method predicts peak positive pressures that are $20 \%$ smaller, peak negative pressures that are $10 \%$ larger, and a full width at half maximum that is $45 \%$ larger.
High-intensity focused ultrasound simulations for an axisymmetric transducer in water have even larger variations in peak positive pressures and intensity but smaller variations in peak negative pressure. Simulations for a rectangular transducer in tissue, where absorption is more prominent, exhibit smaller but significant variations in peak pressures and intensity. Satisfying the Rankine-Hugoniot condition plays an important role in numerical simulations of the KZK equation. [This work was supported by NIH Grants R01-HL075485 and 1R01-CA114093-02.]

\section{$1: 30$}

2pBBa3. Shock-wave energy deflection due to the presence of bone. Thomas Matula, Juan Tu, Michael Bailey (Appl. Phys. Lab, Univ. of Washington, 1013 NE 40th St., Seattle, WA 98105), Kirsten Fagnan, and Randy LeVeque (Univ. of Washington, Seattle, WA 98105)

The physical interaction of shock waves with musculoskeletal tissues is inherently different from other high-pressure acoustic therapies. Whereas high-intensity focused ultrasound and lithotripsy focus their energy in regions of soft tissue, musculoskeletal shock-wave therapy (SWT) involves focusing shock waves (SWs) near or at bones. The presence of bones will cause reflection, refraction, and diffraction of acoustic energy. In our analysis of cavitation generated during clinical SWT treatment, we noticed that the cavitation was occurring away from the SW focus. We confirmed this by performing an in-vitro experiment on a bone embedded in gel. We also quantified the deflection angle as a function position near a bone in water: A talus was manufactured using rapid prototyping. It was placed near the focus of an electrohydraulic SW device. To visualize the pressure field, a high-speed camera imaged the cavitation field generated around the focus. As the bone was moved closer to the focus, the cavitation field (and thus, the pressure field) deflected away from the bone. The deflection angle was measured as a function of relative distance between the bone and focus. Numerical simulations were performed to model the deflection of energy away from the bone.

\section{$1: 45$}

2pBBa4. A sensitive, broadband polyvinylidine difluoride (PVDF) hydrophone for accurate characterization of shock waves. Adam D. Maxwell, Brian E. MacConaghy (Ctr. for Industrial and Medical Ultrasound, Appl. Phys. Lab., Univ. of Washington, Seattle, WA), Oleg A. Sapozhnikov (Moscow State Univ., Moscow, 119992, Russia), and Michael R. Bailey (Univ. of Washington, Seattle, WA)

Few hydrophones are capable of measuring high-intensity fields such as shock waves accurately. One of the more reliable is the fiber-optic probe hydrophone. However, this system is expensive and insensitive. We 
created a new PVDF hydrophone and compared it with a fiber-optic system. The hydrophone consisted of a $25-\mu \mathrm{m}$-thick PVDF membrane with a 0.5 -mm active element and a preamplifier, which were each held in separate attached polycarbonate housings. The amplifier had adjustable gain and could account for membrane resonance to flatten the frequency response. A model of the frequency response for the system was developed, which agreed well with the measured response. Shock waves were measured in two Dornier HM-3 clones and an electromagnetic lithotripter. Measurements were also recorded using a 2-MHz focused piezoceramic source and a broadband PVDF source. Shock-wave measurements closely matched those recorded by the fiber-optic hydrophone and calculations made with a KZK-type model. Very little damage to the membrane was found after applying several thousand shock waves. This new membrane hydrophone is robust and sufficiently accurate to measure high-intensity fields, while greatly reducing cost, increasing sensitivity, and simplifying measurements of shock waves. [Work supported by NIH DK43881, DK55674, and NSBRI SMS00402.]

\section{2:00}

2pBBa5. Calculation and measurement of acoustic scatter to assess fragmentation in shock wave lithotripsy. Neil R. Owen (Ctr. for Industrial and Medical Ultrasound, Appl. Phys. Lab., Univ. of Washington, 1013 NE 40th St., Seattle, WA 98105), Oleg A. Sapoznikov, Michael R. Bailey, and Lawrence A. Crum (Univ. of Washington, Seattle, WA 98105)

Shock wave lithotripsy (SWL) is currently conducted with little feedback on whether kidney stones are breaking. To determine if fragmentation could be assessed, acoustic scatter from intact and fractured stone models was calculated numerically and measured in vitro. Acoustic scatter from the stones, which were modeled with glass spheres, was calculated numerically using a linear elastic model, initialized with known elastic constants, and propagated from the stone model surface using the Helmholtz-Kirchhoff integral. Experimentally, shock waves were generated with a research lithotripter and scatter was measured with a broadband, spherically focused receiver. Calculated and measured results agreed well in the time domain. In frequency, power spectra were integrated to find energy and showed that scatter from the fractured stone model had higher energy in specific frequency bands that were related to the reverberation period. High-speed photography indicated that cavitation did not adversely affect the analysis of scatter. In this work it was possible to distinguish between the intact and fractured stone models. This method could be applied to stones that fragment gradually under the application of shock waves and potentially be used to estimate fragment size, and therefore the endpoint of therapy. [Work supported by NSBRI SMS00402 and NIH DK43881.]

\section{2:15}

2pBBa6. Bubble responses to lithotripsy shock waves. Wayne Kreider, Michael R. Bailey (Ctr. for Industrial and Medical Ultrasound, APL, Univ. of Washington, 1013 NE 40th St., Seattle, WA 98105), Oleg A. Sapozhnikov (Moscow State Univ., Moscow 119992, Russia), and Lawrence A. Crum (Univ. of Washington, Seattle, WA 98105)

The responses of bubbles subjected to a lithotripsy shock wave have been investigated numerically and experimentally to elucidate the role of heat and mass transfer in the underlying dynamics of strongly excited bubbles. Single spherical bubbles were modeled as gas-vapor bubbles by accounting for liquid compressibility, heat transfer, vapor transport, vapor trapping by noncondensable gases, diffusion of noncondensable gases, and heating of the liquid at the bubble wall. For shock-wave excitations, the model predicts bubble growth and collapse, followed by rebounds whose durations are significantly affected by vapor trapping. To experimentally test these predictions, bubble rebound durations were measured using passive cavitation detectors, while high-speed photographs were captured to evaluate the local cavitation field and to estimate radius-time curves for individual bubbles. Data were acquired for bubbles in water with varying temperature and dissolved gas content. Measurements verify that vapor trapping is an important mechanism that is sensitive to both temperature and dissolved gas content. While this work focuses primarily on individual bubbles, some bubble cloud effects were observed. Analysis with a simple multibubble model provides noteworthy insights. [Work supported by NIH T32-EB001650, NIH DK43881, and NSBRI SMS00402.]

\section{$2: 30$}

2pBBa7. Millisecond initiation of boiling by high-intensity focused ultrasound in tissue-mimicking phantoms. Michael S. Canney, Michael R. Bailey (Ctr. for Industrial and Medical Ultrasound, APL, Univ. of Washington, 1013 NE 40th St., Seattle, WA 98105-6698), Vera A. Khokhlova (Moscow State Univ., Moscow 119992, Russia), and Lawrence A. Crum (Univ. of Washington, Seattle, WA 98105-6698)

Nonlinear propagation effects leading to shock formation at the focus of high-intensity focused ultrasound (HIFU) treatments can accelerate heating and cause rapid boiling in tissue. Boiling can be utilized for targeting the treatment with B-mode ultrasound and should be taken into account when planning the treatment, because bubbles reflect ultrasound and thereby displace and distort the lesion shape. In these experiments, an HIFU transducer of 2-MHz frequency, $4-\mathrm{cm}$ aperture, and $4.5-\mathrm{cm}$ focal length was used to investigate heating effects from shock formation in tissue-mimicking phantoms. The time required to attain $100{ }^{\circ} \mathrm{C}$ at the focus was calculated with weak shock theory from the peak amplitudes calculated with a KZK-type model, and time to boiling was measured by high-speed video and a 20-MHz passive cavitation detector (PCD) for different values of phantom absorption (both lower than tissue absorption) and HIFU power (100-200 W). Boiling was observed in $3 \mathrm{~ms}$ at the highest power level used by the observation of visible bubbles and by a significant change in the PCD time signal and spectrum. [Work supported by NIH DK43881, NSBRI SMS00402, and RFBR.]

\section{2:45}

2pBBa8. Characterization of the YDME FEP-BY02 tumor therapy system. Bryan Cunitz (Ctr. for Industrial and Medical Ultrasound, Appl. Phys. Lab., Univ. of Washington, Seattle, WA 98105), Stuart Mitchell, Yak-Nam Wang, Barbrina Dunmire, and Peter Kaczkowski (Univ. of Washington, Seattle, WA 98105)

The YDME FEP-BY02 is an extracorporeal tumor therapy device that has been in clinical use in China for several years. In the USA, this device is currently under investigation for the treatment of pancreatic cancer. Prior to being submitted for an application for an Investigation Device Exemption (IDE) with the FDA, the device will be required to undergo a thorough characterization of all systems involved in patient treatment. The purpose of this research is to characterize the FEP-BY02 and obtain data for an IDE application to the FDA for the treatment of pancreatic cancer using the FEP-BY02. A series of acoustical characterization, in vitro, and ex vivo tests are being conducted to validate the company's preclinical and clinical experience in China. Comparisons between three methods (radiation force balance, fiber-optic hydrophone, and scattering hydrophone) for measuring/determining focal intensities will be used to assess the validity of the measurement techniques and evaluate acoustic output over the functional input range of the FEP-BY02. Transparent gels impregnated with thermally sensitive markers will be used to visualize lesion formation and evaluate lesion characteristics with respect to input parameters. Thermocouples implanted in tissue mimicking thermal gels and ex vivo tissue will be use to evaluate the thermal response with respect to input parameters. Preliminary data indicate that the therapeutic ultrasound treatment system forms consistent lesions at the desired target.

\section{3:00}

2pBBa9. Acoustic virtual laboratory: A modeling tool for high-intensity focused ultrasound applications. Francesco P. Curra, Steven G. Kargl, and Lawrence A. Crum (Ctr. for Industrial and Medical Ultrasound, Appl. Phys. Lab., Univ. of Washington, Seattle, WA)

The field of high-intensity focused ultrasound (HIFU) is emerging with strong potential and broad medical applications. Characterized by its ability to penetrate at depth inside the body without harming intervening tissue, therapeutic ultrasound has posed the basis for a new array of nonin- 
vasive therapies. However, the inherent complexity of biological media and the nonlinear nature of ultrasound propagation at HIFU regimes make optimization and control of the therapy still a challenging task. In this respect, an accurate modeling tool, the Acoustic Virtual Laboratory (AVL), will be presented for solving multidimensional HIFU problems in complex geometries that can greatly assists in predicting HIFU applications effects and, therefore, help in the optimization and control of the treatment. AVL consists of 2-D, 2.5-D (cylindrical symmetry), and 3-D coupled solutions for acoustic and elastic wave propagation in heterogeneous, lossy, bubbly, and porous media with the bioheat equation for temperature estimation. It includes linear and nonlinear wave propagation, arbitrary frequency power law for attenuation, and can account for multiple reflections and backscattered fields. Sample results for tumor ablation, acoustic hemostasis, and propagation through porous bones will be illustrated. [Work supported by US Army Medical Research Acquisition Activity and NIH NCRR Grant R21 RR 21472.]

WEDNESDAY AFTERNOON, 29 NOVEMBER 2006

KAHUKU ROOM, 3:30 TO 5:45 P.M.

\title{
Session 2pBBb
}

\section{Biomedical Ultrasound/Bioresponse to Vibration: Biomedical Imaging and Microscopy}

\author{
Suk Wang Yoon, Cochair \\ Sung Kyun Kwan Univ., Dept. of Physics, 300 Chunchun dong, Jangan Ku, Suwon 440-746, Rep. of Korea \\ Yoshifumi Saijo, Cochair \\ Tohoku Univ., Dept. of Medical Engineering and Cardiology, 4-1 Soiryomachi, Aoba-ku, Sendai, 980-8575, Japan
}

\section{Contributed Papers}

\section{3:30}

2 pBBb1. Temperature dependence of acoustic properties of human tissues with a high-resolution ultrasound backscatter microscope. Jee Woo Park (Acoust. Res. Lab., Dept. of Phys., SungKyunKwan Univ., Suwon 440-746, Republic of Korea), Jiehui Liu, Byoung Nam Kim, and Suk Wang Yoon (SungKyunKwan Univ., Suwon 440-746, Republic of Korea)

The B-mode in vitro images of human skin and ocular tissues are obtained using a high-resolution ultrasound backscatter microscope (UBM) at the frequencies of 40, 80, and $100 \mathrm{MHz}$. It shows that a highresolution UBM can visualize more detailed superficial microstructures of skin and eye at higher frequencies. In order to improve high-frequency ultrasound images, we have investigated the acoustic properties, such as sound velocity, sound attenuation, and backscatter coefficient, of human tissues with this UBM system over the frequency range from 25 to 110 MHz. Their acoustic properties are also investigated as a function of temperature in the range from $20^{\circ} \mathrm{C}$ to $65^{\circ} \mathrm{C}$, which may provide bases for ultrasound image monitoring of thermal therapy. [Work supported by Strategic National R\&D Program of Ministry of Commerce, Industry and Energy (MOCIE), Republic of Korea.]

\section{3:45}

2pBBb2. Calibration for biological acoustic impedance microscope. Naohiro Hozumi (Aichi Inst. of Technol. 1247 Yakusa-cho, Toyota, 470-0392 Japan), Satoshi Terauchi, Ayumi Kimura, Masayuki Nagao, Sachiko Yoshida (Toyohashi Univ. of Technol.), Kazuto Kobayashi (Honda Electron.), and Yoshifumi Saijo (Tohoku Univ.)

The presentation deals with the calibration for a biological acoustic impedance microscope. A block of tissue was placed on a plastic substrate, and an acoustic pulse focused on the interface was transmitted from the rear side of the substrate. The reflection was received by the same transducer and interpreted into local acoustic impedance. An acoustic impedance image was obtained by mechanically scanning the transducer. A reference material was simultaneously observed in order to perform calibration. A water droplet was employed as a reference material, however, it often entered into the interface between the substrate and tissue, leading to the reduction of the image quality. Therefore, several kinds of compounds were investigated. Silicone rubber was relatively homogeneous and stable in acoustic impedance, after a certain period of curing time. However, its dispersion with the scale of focal spot size was as large as $0.02 \mathrm{Ns} / \mathrm{cm}^{3}$, suggesting that a sufficient number of points should be measured and averaged in order to obtain a precise reference signal. Finally, change in local acoustic impedance of cerebella tissue of a rat as a function of postnatal days was quantitatively assessed for each independent layer like molecular layer, granular layer, and white matter.

\section{4:00}

2pBBb3. Ultrasonic evaluation of blood coagulation from stroke patients. Ying Li, Dong-Guk Paeng (Oceanic Information and System Eng., Ocean College, Cheju Natl. Univ., JEJU, Korea, 690-756), Sitaramanjaneya Anjaneya Reddy Guntur, Min Joo Choi, Young Ree Kim, and Jae-Chul Choi (Cheju Natl. Univ., JEJU, Korea, 690-756)

It is already known that the ultrasound speed and attenuation coefficient are increasing during the blood clotting process. A pulse echo system was set up to measure the sound speed and attenuation coefficient of human blood at $37 \mathrm{C}$. From ten healthy volunteers, the blood was taken to measure sound speed and attenuation coefficient during blood coagulation without and with heparin. For ten stroke patients, sound speed and attenuation were measured with aPTT or PT for comparison. The variation of sound speed and attenuation coefficient of blood after adding heparin was small. Sound speed of plasma from healthy volunteers was increased to 7 from $1535 \mathrm{~m} / \mathrm{s}$ over $50 \mathrm{~min}$ without heparin, but to $1 \mathrm{~m} / \mathrm{s}$ during the first $5 \mathrm{~min}$ for heparin-added blood. Sound speed of plasma from stroke patients was changed little compared to that from healthy volunteers without heparin. Attenuation coefficient was increased linearly during plasma coagulation for both volunteers and stroke patients, but its temporal slopes were much smaller for stroke patients. The blood samples from the stroke patients that investigated how heparin affects sound speed and attenuation as a function of time before and after taking the heparin and the results will be discussed.

\section{$4: 15$}

2pBBb4. Correlation between the degree of hemolysis and acoustic impedance, speed of sound, and attenuation in flowing whole blood. Adam White and Constantin-C. Coussios (Inst. of Biomed. Eng., Dept. of Eng. Sci., Univ. of Oxford, 17 Parks Rd., Oxford OX1 3PJ, UK, constantin.coussios@eng.ox.ac.uk)

The effect of increasing levels of mechanical hemolysis on the propagation of $15-\mathrm{MHz}$ ultrasound in flowing whole blood at $37^{\circ} \mathrm{C}$ was investigated. An experimental setup was developed, which enabled the continuous measurement of attenuation, speed of sound, and acoustic impedance 
in porcine whole blood under conditions of laminar flow and mean shear rates well above the threshold for red cell aggregation. Starting with an initial hematocrit of $35 \%$, an increasing volume fraction of the blood in the flow loop was hemolyzed by exposure to very high shear stresses. The attenuation coefficient at $15 \mathrm{MHz}$ was found to vary between $1 \mathrm{~dB} / \mathrm{cm}$ for $0 \%$ hemolysis (no red cell damage) to $5 \mathrm{~dB} / \mathrm{cm}$ for $75 \%$ hemolysis, remaining approximately constant for higher hemolysis levels. Speed of sound decreases from $1561 \mathrm{~m} / \mathrm{s}$ for healthy blood to $1545 \mathrm{~m} / \mathrm{s}$ for $80 \%$ hemolysis. Finally, acoustic impedance was also found to vary from a minimum of $1575 \mathrm{Kg} / \mathrm{m}^{2}$ to $1589 \mathrm{Kg} / \mathrm{m}^{2}$ between $15 \%$ and $90 \%$ hemolysis. These results show great promise towards solving the inverse problem, namely developing a technique capable of deducing the degree of hemolysis noninvasively and in real time based on measured changes in ultrasound parameters.

\section{4:30}

2pBBb5. Multimodality tissue-type imaging of prostate cancer. Ernest Feleppa, Shreedevi Dasgupta, Sarayu Ramachandran (Riverside Res. Inst., 156 William St., New York, NY 10038, feleppa@rrinyc.org), Christopher Porter, David Dail, and Marc Lacrampe (Virginia Mason Medical Ctr., Seattle, WA 98101)

Unlike many tumors, prostate cancer cannot be imaged reliably by any single, existing, conventional modality. This makes guiding biopsies, targeting therapy, and monitoring effects problematic. Therefore, our objective is to improve significantly the depiction of prostate cancers using multimodality, multifeature techniques combined with nonlinear classifiers such as artificial neural networks (ANNs) and support vector machines (SVMs). Our previous studies show encouraging potential for tissue typing and imaging based on ultrasound spectrum analysis using ANNs and SVMs that produce ROC-curve areas of 0.84 to 0.88 compared to 0.64 for conventional ultrasound imaging. Similar studies by others have shown that magnetic-resonance spectroscopy can produce comparable ROCcurve areas based on the ratio of choline and creatine to citrate. Because these two modalities sense entirely distinct properties of tissue, i.e., microscopic mechanical properties by ultrasound and chemical properties by magnetic resonance, their combination should markedly improve classification performance in distinguishing between cancerous and noncancerous prostate tissue. We have undertaken preliminary studies incorporating these two modalities. These studies demonstrate the feasibility of coregistering features from each modality and generating new, combinedmodality images that exploit the attributes of both modalities.

\section{$4: 45$}

2pBBb6. Validating the theoretical framework relating ultrasonic spectrum-analysis parameters to scatterer properties. Shreedevi Dasgupta, Ernest Feleppa, Jonathan Mamou (Riverside Res. Inst. 156 William St., NY 10038), and Mark Rondeau (Cornell Univ., Ithaca, NY)

Existing theoretical frameworks for ultrasound spectrum analysis relate properties of backscattered echo signals from tissues to scatterer properties. However, the reliance of these frameworks on numerous key assumptions may not be appropriate for evaluating tissue properties. The objective of our studies is to validate those assumptions or reduce dependence on them so that estimates of scatterer properties can be made with greater confidence. Ultrasound rf data were acquired from isolated, uveal melanoma cells suspended in liquid media using center frequencies of 10 and $35 \mathrm{MHz}$. Average cell size was $12 \mu \mathrm{m}$ and concentrations ranged from $1.3 \times 10^{8}$ cells $/ \mathrm{ml}$ to $2.0 \times 10^{6}$ cells $/ \mathrm{ml}$. Theoretical spectralparameter values predicted by the Lizzi framework were calculated using known values for size and concentration and published values for sound propagation velocity and acoustic impedance. Normalized spectral parameters were computed from the experimental rf data using standard Fourier methods. Predicted and experimental values for the slope, midband, and intercept parameters agreed to within $11 \%$. Initial results tend to validate the theory at these scatterer concentrations. Future work will provide more insight into scattering behavior under a greater variety of conditions, e.g., scatterer sizes and concentrations.
2pBBb7. Alias-free estimation of blood velocity using wideband pulse transmission. Naohiko Tanaka (Faculty of Systems Eng., Shibaura Inst. of Technol., 307 Fukasaku, Saitana 337-8570, Japan, tanaka@se.shibaura-it.ac.jp)

Blood velocity information that is obtained from a pulsed Doppler system is applied to clinical diagnoses of the circulatory system. However, the conventional pulsed Doppler technique has a fundamental limitation on Doppler velocities: blood velocity is measured erroneously when it is greater than the Nyquist limit. This phenomenon is called aliasing. This study is aimed at expanding the range of measurable blood velocities while avoiding aliasing. A new technique proposed here is based on wideband pulse transmission and phase correction. A series of short pulses with a wide frequency band is transmitted in this technique. Received echoes are Fourier transformed and converted to the cross spectrum. The phase of the cross spectrum is proportional to the frequency and the blood velocity. When the blood velocity is greater than the Nyquist limit, a part of the phase of the cross spectrum is larger than $\pm \pi$ and is wrapped in $\pm \pi$. The wrapped phase data are corrected using wideband echo information to avoid aliasing. Then the gradient of the corrected phase is estimated and converted to the blood velocity. Simulation results show that the proposed technique triples the velocity range.

\section{$5: 15$}

2pBBb8. Continuous delay estimation with polynomial splines. Gianmarco Pinton and Gregg Trahey (Duke Univ., 136 Hudson Hall, Box 90281, Durham, NC 27708, gfp@duke.edu)

Delay estimation is used in biomedical ultrasonics to determine blood flow, phase aberration corrections, and elastographic images. The accuracy of the algorithms developed to determine these delays depends on noise, bandwidth, and delay range. Subsample delay resolution is required in most cases. Two new algorithms that use cubic polynomial splines to continuously represent the delay and the apply pattern matching functions are introduced. The continuous analytic expressions for the pattern matching functions are used to calculate the delay. Normalized cross correlation and the sum of the squared error are implemented. These algorithms are compared to conventional delay estimators, such as discrete normalized cross correlation, autocorrelation, and to another spline-based method. Simulations compare the performance across noise, delay, and bandwidth. The proposed algorithms perform better, in terms of bias and jitter. For example, the jitter at a 0.5 subsample shift is $2.3 \times 10^{-3}$ subsample shifts for interpolated normalized cross correlation, and $3.5 \times 10^{-4}$ and 4.0 $\times 10^{-4}$ for the two proposed algorithms. The computational requirements of the new algorithms are also considered and are shown to be competitive with the interpolated normalized cross correlation at low interpolation values and smaller at large interpolation values. [This work was supported by NIH grants R01-HL075485 and 1R01-CA114093-02.]

\section{$5: 30$}

2pBBb9. Erythrocyte aggregability from human, horse, and rat blood by ultrasound backscattering measurements. Kwon-Ho Nam (Interdisciplinary Postgraduate Program in Biomed. Eng., Cheju Natl. Univ., 66 Jejudaehakno, Jeju-si Jeju-do, South Korea, 690-756), DongGuk Paeng, Tae-Hoon Bok, and Min Joo Choi (Cheju Natl. Univ., 66 Jeju-si Jeju-do, South Korea, 690-756)

A pulse-echo system was used in a cylindrical chamber with a stirring magnet and a $5-\mathrm{MHz}$ focused transducer for studying erythrocyte aggregation from human, horse, and rat blood. The highest backscattering power was measured from horse blood and followed by human and rat blood both at stasis and under shear forces. In human and horse blood, the backscattered power (BP) was decreased with stirring speed and saturated within $1 \mathrm{~min}$. The BP was increased to $20 \mathrm{~dB}$ when blood flow was stopped. Since the aggregation of rat erythrocytes in autologous plasma is very low and barely detectable by the ultrasound $\mathrm{BP}$, rat plasma was 
replaced by phosphate buffered saline containing polyvinylpyrrolidone 360 in order to enhance the aggregation. In conclusion, this simple in vitro ultrasound measurement system requires a small amount of blood, which may provide an alternative method to investigate the mechanisms and physiological roles of erythrocyte aggregation. Furthermore, the rat erythrocytes in polymer solution may be useful for an automatic measurement system to examine the bioactive materials that affect aggregation in preclinical studies.

WEDNESDAY AFTERNOON, 29 NOVEMBER 2006

OAHU ROOM, 1:00 TO 4:45 P.M.

\title{
Session 2pEA
}

\section{Engineering Acoustics: Ultrasonic Functional Devices II}

\author{
Gary W. Elko, Cochair \\ mh acoustics LLC, 26 Blackburn Pl., Summit, NJ 07901 \\ Jun Kondoh, Cochair \\ Shizuoka Univ., Graduate School of Science and Technology, Johoku 3-5-1, Hamamatsu-shi, Shizuoka 432-8561, Japan
}

\section{Invited Papers}

1:00

2pEA1. Development of shear-horizontal-surface-acoustic wave (SH-SAW) sensor for liquids. Jun Kondoh (Grad. School of Sci. and Technol., Shizuoka Univ., Johoku 3-5-1, Hamamatsu-shi, Shizuoka 432-8561, Japan) and Showko Shiokawa (SAW\&SPR-Tech. Corp., Hamamatsu-shi, Shizuoka 432-8011, Japan)

Acoustic-wave devices have been applied as chemical and physical sensors for gas and liquid phases. Since the surface acoustic wave (SAW) on a $36^{\circ}$-rotated Y-cut X-propagating $\mathrm{LiTaO}_{3}$ single crystal (36YX.LT) is in the shear horizontal (SH) mode, SH-SAW devices have been applied as liquid-phase sensor. In this paper, we present fundamental properties of the SH-SAW sensor and its applications. When a liquid is loaded on the SH-SAW propagation surface, evanescent fields of the SH displacement and piezoelectric potential penetrate into the liquid. Evanescent fields are influenced from the liquid, so phase velocity and amplitude of the SH-SAW are perturbed. By detecting the change, liquid-phase sensor is realized. Based on the theoretical calculation, the SH-SAW sensor is influenced by liquid properties, i.e., density and viscosity products, relative permittivity, and conductivity. For simultaneous detection of these properties, a three-channel SH-SAW sensor is fabricated. Evaluation of mixed solutions of glucose and ethanol is carried out using the sensor. The results indicate that the three-channel SH-SAW sensor can be applied to a fermentation monitoring system.

\section{$1: 20$}

2pEA2. Lead zirconate titanate thick-film ultrasonic transducer for 1- to $50-\mathrm{MHz}$ frequency bands fabricated by hydrothermal method. Mutsuo Ishikawa, Minoru Kurosawa (Interdisciplinary Grad. School of Sci. and Eng. Tokyo Inst. of Technol. Kurosawa lab. G2-32, 4259 Nagatutamati, Midori-ku, Yokohama, Kanagawa 226-8502), Tomohito Hasegawa, Hiroshi Kitsunai, Akito Endoh, Shinichi Takeuchi (Toin Univ. of Yokohama), Takeyoshi Uchida, and Tsuneo Kikuchi (Natl. Inst. of Adv. Industrial Sci. and Technol.)

This prototype ultrasonic transducer had about a 30- $\mu \mathrm{m}$ thick hydrothermal PZT layer on a 50- $\mu \mathrm{m}$ thick titanium substrate. An active area of this transducer was $3.5 \mathrm{~mm}$ in diameter. Material constants of this hydrothermal PZT thick film were measured. Electromechanical coupling coefficient kt was over 0.25 and acoustic impedance was 6 Mrayl. A mechanical resonance frequency in the thickness mode of the prototype ultrasonic transducer was approximated by a mason's equivalent circuit using the thickness and the material constants of the hydrothermal PZT film and the Ti substrate. The prototype ultrasonic transducer had a double broad peak spectrum of about 15 and $30 \mathrm{MHz}$ in simulation. Also, these broad peaks were a half wavelength and a quarter wavelength resonance frequency in a combined structure of the hydrothermal PZT thick film and the Ti substrate. The prototype ultrasonic transducer transmitted ultrasound in water. Experimental results were the prototype ultrasonic transducer radiated ultrasound at the frequency range from 1 to $50 \mathrm{MHz}$ as wideband using respective resonance frequency. A reason for the wideband characteristics radiation of ultrasound was the prototype ultrasonic transducer has low acoustic impedance and double broad peak.

\section{Contributed Papers}

1:40

2pEA3. Equivalent network representation for length-extensional vibration modes in a side-plated piezoelectric bar with a varying parameter. Ken Yamada (Dept. of Electron. Eng., Faculty of Eng., Tohoku Gakuin Univ., 1-13-1 Chuo, Tagajo 985-8537, Japan)

Equivalent network representation is derived for length-extensional vibration modes in a side-plated piezoelectric bar with varying parameters along it. In contrast to findings of a former report [Yamada et al., IEEE Trans. UFFC 48(2), 613-616 (2001)], which dealt with the longitudinal piezoelectric effect, the bar in this study is presumed to be excited through a transverse piezoelectric effect. The network comprises an electrical transmission line of length corresponding to the bar length and an electrical port with a capacitor that is linked to the line at both its ends via two ideal transformers. Frequency characteristics of the input electric admittance of the bar with a sinusoidally varying piezoelectric parameter, which shows the unique feature of the vibrator, is demonstrated using equivalent network analysis. The possibility of applying this network for modeling the interdigital transducer for excitation and detection of surface acoustic waves (SAWs) is discussed. 
2pEA4. Measuring micro liquid volume by acoustic resonant technique. Makoto Wakabayashi, Daisuke Koyama, Kentaro Nakamura, and Sadayuki Ueha (P\&I Lab. Tokyo Technol., 4259-R2-26, Midori-ku, Yokohama, Kanagawa 226-8503, Japan)

A method to measure micro liquid volume less than $300 \mathrm{ln}$ with the accuracy of $0.1 \%$ is highly needed in biogenetic and biochemical industries. The authors propose a novel method to estimate micro liquid volume in a small well using acoustic resonance. In our experiments, liquid is in a container $6.5 \mathrm{~mm}$ in inner diameter and $9 \mathrm{~mm}$ in height, and the sound pressure near the well is measured by sweeping the illuminating sound field from 6 to $11 \mathrm{kHz}$. According to the relationship between the resonance frequency and the volume, we can successfully estimate the small volume with the accuracy of $0.1 \%$ and an excellent repeatability. For simultaneous multipoint measurements, we experimentally investigate the effect of adjacent wells on resonant frequency of the target well. It is revealed that the resonance frequency shift due to the mutual coupling between the adjacent wells is negligible if the well interval is less than 5 $\mathrm{mm}$.

\section{2:10}

2pEA5. A multidimensional overview for ultrasonic motor driven loudspeaker development. Hirokazu Negishi, Juro Ohga (Dept. of Electron. Systems Eng., Essex Univ., 1-37-14 Koyabe Yokosuka 238-0026, Japan, hiro.note@jcom.home.ne.jp), and Juro Ohga (Shibaura Inst. of Technol., Tokyo 108-8548, Japan)

Twelve years of history for the development activity of the ultrasonic motor driven loudspeaker show a multidimensional aspect. Three main axes are geography, frequency, and mechanical impedance. Geography came first since initial conception was in the UK, yet the prototyping was in Japan. The origin was an idea for a stepping motor driven digital loudspeaker that was to address improving abnormalities coming from the fundamental resonant frequency, the so-called "F0." Consultation revealed the idea was weak but unexpectedly "ultrasonic motor" was recommended. This led the prototyping to be in Japan, where the invention and development of the motor took place. The second is frequency. For audible sound reproduction, inaudible ultrasonic frequency is employed. Although combination of these two regions in frequency is not new for sound reproduction, a couple between the bottom end of the audible sound reproduction and the ultrasonic motor is completely new for this arena. The third is mechanical impedance since convention is low whereas this one is high. Actually, this is the core of the attempt as most abnormalities around "F0" stem from weak mechanical impedance. Other axes also exhibit contrast against conventions such as linearity, controlling, driving, and lifetime of the motor itself, as well as configuration of the sounding mechanism.

\section{2:25}

2pEA6. Loudspeaker using continuous revolution of a piezoelectric ultrasonic motor. Yuta Ohnuma, Yusuke Iwaki, Juro Ohga (Shibaura Inst. of Technol., 3-7-5, Toyosu, Koto-ku, Tokyo 135-8548, Japan, m106020@sic.shibaura-it.ac.jp), Hirokazu Negishi (Essex Univ., Yokosuka 238-0026, Japan), and Kazuaki Maeda (TOA Corp., Japan)

Volume displacement of a direct radiator loudspeaker diaphragm is inversely proportional to the square of its signal frequency. The lowest frequency of a flat response is restricted by the diaphragmfs fundamental resonance frequency. Therefore, a loudspeaker diaphragm for radiation of a low-frequency signal shall have large amplitude and low resonant frequency. The authors propose the application of a piezoelectric ultrasonic motor for this purpose. It produces a completely controlled large output force because its output mechanical impedance is much greater than any conventional transducer or motor. An ultrasonic motor whose stator is connected to a direct-radiator loudspeaker cone diaphragm by a rod and whose rotor is loaded by a heavy metal ring rotates with a constant veloc- the diaphragm because a heavy ring with large inertia tends to rotate with constant velocity. This paper reports construction of a practical experimental model and its output sound pressure characteristics.

\section{2:40-3:00 Break}

\section{3:00}

2pEA7. Removal of thin hard coating on glass substrate by collision of nanometer-sized particles accelerated in high-intensity ultrasonic field. Naoya Kudo and Kazunari Adachi (Faculty of Eng., Yamagata Univ., 4-3-16 Jounan, Yonezawa, Yamagata, 992-8510 Japan, ere69123@dip.yz.yamagata-u.ac.jp)

The aim of this study is to establish a fundamental recycling technology for optical lenses. In industry, a common conventional technique for cleaning the surface of a small object such as an optical lens is through the use of ultrasonic cavitation. However, the intensity of shock waves caused by the cavitation is difficult to control. The authors have considered that the substances that adhere to lens surfaces, such as hard optical coatings and stains, are removable using collisions, against the lens surface, of many tiny particles that are accelerated by a high-intensity ultrasonic field. Some physical parameters that dominate the removal effect have been changed systematically to illustrate the surface-refreshing process for lenses in the experiments. The authors worked to experimentally clarify the particle collision effect on the removal. In addition, the acoustic field was analyzed using finite-element method. The authors have also attempted to elucidate the principle of the removal technique through comparison of experimental results with those obtained through numerical simulation. A precise elucidation, however, remains as an important problem to be solved.

\section{3:15}

2pEA8. Atomization of high-viscosity materials by one-point convergence of sound waves radiated from an aerial ultrasonic source using a square transverse vibrating plate. Hikaru Miura (Dept. of Elec. Eng., College of Sci. and Technol., Nihon Univ., 1-8-14, Kanda Surugadai, Chiyoda-ku, Tokyo 1018308, Japan)

It is difficult to atomize high-viscosity materials such as oil without contact; however, it is possible to do so using intense aerial ultrasonic waves. As a sound source for that purpose, an aerial ultrasonic source with $20-\mathrm{kHz}$ resonance frequency was used along with a square transverse vibrating plate that has the nodal mode of vibration amplitude in the shape of a lattice. The source radiates four-directional sound waves in the far field. To obtain high sound pressure in the near field, radiated sound waves were converged using several flat boards and four parabolic reflective boards placed near the source. Sound pressure reaches $6.5 \mathrm{kPa}$ (S.P.L. 170 $\mathrm{dB}$ ), a very high level, at the convergent point. Scattering and atomization of the high-viscosity materials were examined at the point of convergence. Results show the following. Atomization by a sound wave is possible in the case of high-viscosity materials. The higher the sound pressure, the shorter the beginning time of atomization becomes. The distribution of the atomized particles diameter is $0.05-0.22 \mathrm{~mm}$ at the movement viscosity of $10000 \mathrm{cSt}$.

2pEA9. Ultrasonic complex vibration converters using different sound velocity metal ring pairs with slanted thickness. Jiromaru Tsujino, Tetsugi Ueoka, Goh Kishimoto, and Rohei Karatsu (Kanagawa Univ., 3-27-1 Rokkakubashi, Kanagawa-ku, Yokohama 221-8686, Japan)

The ultrasonic complex vibration systems are effective for various high power applications. New complex vibration converters using different sound velocity metal ring pairs with slanted thickness are proposed and studied. Two different sound velocity rings with a slanted surface along the diameter are superimposed. The converter ring pair may be installed in any place of a longitudinal vibration system and driven by a longitudinal vibration source. The system is driven transversally according 
to traveling time difference between both sides of the converter. The vibration rod vibrates in elliptical to circular locus in the case where these two natural frequencies are adjusted as phase difference is near to $90 \mathrm{deg}$. Thus, various one-dimensional complex vibration systems are obtained. Piezo-ceramics cut or polarized slanted may be used. The 18-mm-diam converter for $27-\mathrm{kHz}$ system consists of a phosphor bronze and stainless steel rings. The rings are cut slanted, 1.5 to $6.5 \mathrm{~mm}$ in thickness at outer diameter, and superimposed to an 8-mm-thick ring. Two or three converters are available for more complicated vibration modes. Using 27 - and $150-\mathrm{kHz}$ complex vibration systems, 1.0 -mm-thick aluminum plates and coated thin copper wires for IC cards were welded directly with material strength.

\section{$3: 45$}

2pEA10. Nondestructive inspection system for corrosion detection in fuel tank liners. John W. Doane, Michael D. Gray, and Jayme J. Caspall (Georgia Inst. of Technol., 771 Ferst Dr., Atlanta, GA 30332, jd37@mail.gatech.edu)

An ultrasonic, nondestructive inspection system for the early detection of corrosion in fuel tank aluminum liners was developed and tested. A Labview-controlled scanner, which rode on the tank's resident rib braces, housed a $10-\mathrm{MHz}$ transducer and a miniature video camera. The bidirectional (axial, azimuthal) scan resolution was controlled by adjusting the surface standoff distance of the focused transducer to produce variable-area interrogation sites. The phenomenon of increased backscatter from corrosion-roughened surfaces was exploited to detect the presence of corrosion in its early stages. The transducer acted as both transmitter and receiver, capturing the echoes from the subject surface while the video camera captured an image of the surface under acoustic inspection. The acoustic and visual data were overlayed to form a composite image of the entire inspection surface. The system was field-tested using two tanks pulled from active duty at the Marine Corps maintenance facility in Cherry Point, NC. [Work supported by JCAA.]

\section{4:00}

2pEA11. Evaluation of the static stress exerted on the clamping bolt in the structure of a bolt-clamped Langevin-type transducer. Toru Takahashi and Kazunari Adachi (Adachi Lab., 8-312, Faculty of Eng., Yamagata Univ., 4-3-16 Jonan, Yonezawa, Yamagata, 992-8510, Japan, g02239@dipfr.dip.yz.yamagata-u.ac.jp)

Bolt-clamped Langevin-type transducers (BLTs) must possess various characteristics depending on the field in which they are used. It has already been clarified by the authors that prestress or bearing stress imposed on piezoelectric elements in a transducer should be sufficiently large to compensate for their low tensile strength. For this study, we measured the strain on the clamping bolt surface using strain gauges pasted on it to evaluate the static prestress that changes with the turning angle of the metal block in clamping. We calculated the strain for some BLTs of identical shape using the finite-element method (FEM) and measured it to confirm the validity of the prestress calculation that was achieved previously by the authors. Unfortunately, a large difference in the strain between the calculated and measured results was observed. We cited three possible reasons for that huge discrepancy. Among them, plastic deformation of the screw thread might most influence the result. As long as the design problem of BLTs pertaining to the prestress remains unresolved, we cannot take full advantage of the excellent electromechanical transduction efficiency of BLTs for high-power ultrasonic applications.

\section{$4: 15$}

2pEA12. Multilayered high-performance transducer using polyurea thin film. Marie Nakazawa, Masaya Tabaru, Daisuke Koyama, Kentaro Nakamura, Sadayuki Ueha (Tokyo Tech., 4259 R2-25, Nagatsuta, Midori-ku, Yokohama, Kanagawa 226-8503, Japan, nakazawa@sonic.pi.titech.ac.jp), and Akihiro Maezawa (Konica Minolta M.G. INC., Tokyo, Japan)

Polyurea thin film exhibits useful characteristics as a high-frequency ultrasonic transducer such as a low acoustic impedance of $70 \%$ of that of PVDF, considerably high piezoelectric constants compatible with conventional piezoelectric polymers, and a higher Curie temperature. A polyurea thin film transducer working at over $100 \mathrm{MHz}$ can be made on any surfaces through vapor deposition polymerization with a high controllability of thickness. To enhance the transducer performance, a multilayered configuration is studied in this study. A special twin-vacuum-chamber is used for laminating the polyurea layers and the aluminum electrodes alternately. We fabricate two- and three-layered transducers with $1.5-\mu \mathrm{m}$ polyurea thin films as well as single-layer transducers. To evaluate the transducer performances, we measure the capacitances, the electrical admittances, the coupling factors, and the Q values. The pulse/echo measurements are also conducted to determine the transmission and receiving characteristics of the transducers using a glass plate as a reflector. The results of the pulse/ echo experiments show that the received voltage amplitude of the multilayered transducer is higher than that of the single-layer transducer.

\section{$4: 30$}

2pEA13. Analysis of fluid-borne coupling in transducer arrays. Kassiani Kotsidou and Charles Thompson (Ctr. for Adv. Computation and Telecommunications, Univ. of Massachusetts Lowell, 1 Univ. Ave, Lowell, MA 01854)

In this work, we will analyze fluid-borne coupling between adjacent elements of a transducer array. In the case of a transducer having low inertia mass, such coupling can result in a reduction in the radiation efficiency and the directional fidelity of the array. The coupling factor and the mutual radiation impedance have been investigated by Lord Rayleigh (1903), Wolff and Malter (1929), and R. I. Pritchard (1960) for the case of an array comprised of ideal piston radiators. These results will be used as a starting point for our work. The effect of the inertia impedance of the transducer and the control of the special distribution of the radiated field is considered. 
IAO NEEDLE/AKAKA FALLS ROOM, 1:00 TO 3:30 P.M.

\title{
Session 2pEDa
}

\section{Education in Acoustics: Education in Acoustics for Children}

\author{
Takayuki Arai, Cochair \\ Sophia Univ., Dept. of Electrical and Electronics Engineering, 7-1 Kioi-cho, Chiyoda-ku, Tokyo 102, Japan \\ Ralph T. Muehleisen, Cochair \\ Illinois Inst. of Technology, Civil and Architectural Engineering, 3201 S. Dearborn St., Chicago, IL 60616
}

Chair's Introduction-1:00

Invited Papers

1:05

2pEDa1. Sound education in urban environment. Yoshio Tsuchida (Kanazawa Inst. of Technol., 7-1 Ohgigaoka, Nonoichi, Ishikawa, 921-8501, Japan)

There are various viewpoints for education and there are various levels for a sound. We have some cases to teach a "sound." A certain person teaches a sound as music, another person teaches it as physics. But education is not teaching. It is more important to feel and enjoy. Then, one's sensitivity for a sound will be nourished. Experimental listening is called Sound Education based on soundscape. The case of a workshop on Sound Education is addressed in this paper. Sound Education finally aims at improvement of our environment. Many activities of Sound Education were devised by R. Murray Schafer [A Sound Education, 100 Exercises in Listening and Soundmaking, Arcana Editions]. Some activities were devised by Joseph Bharat Cornell as Nature Game [Sharing Nature With Children (Sharing Nature Series), Dawn Publications.] Sound Education has several aims. It differs according to the group that sponsors it. Here are the workshops executed by me. One aim for environmental preservation, and another one aim for community design.

2pEDa2. Music as a vehicle to do science in the elementary classroom. Uwe J. Hansen (Dept. of Phys., Indiana State Univ., Terre Haute, IN 47809)

During the past several years, a number of workshops were conducted for public school teachers at the elementary levels. Training and materials were provided to help teachers use music as a vehicle to introduce science concepts. Tools used in these workshops include a long spring to introduce standing waves and the concept of harmonics, a monochord to relate frequency ratios to musical intervals, and simple computer software to introduce wave addition and spectral analysis. All of these will be demonstrated and ways of introducing them in the elementary classroom will be discussed. [These workshops were supported with ASA and Eisenhower funds.]

\section{$1: 45$}

2pEDa3. Exciting demonstration in acoustics by high-school teachers' group: "Stray Cats." Kanako Ueno (Inst. of Industrial Sci., Univ. of Tokyo and Tech. Committee on Education in Acoust. of ASJ, Komaba 4-6-1, Meguroku, Tokyo, 153-8505, Japan), Takayuki Arai, Fumiaki Satoh, Akira Nishimura, and Koichi Yoshihisa (Tech. Committee on Education in Acoust. of ASJ, Meguroku, Tokyo, 153-8505, Japan)

In Japan, to get students interested in a subject, high-school teachers often form a group to share their ideas and inventions on education. "Stray Cats" is one of the most active groups in physics. The group has been proposing many exciting demonstrations, which were contrived to support students' learning process with intuitive understanding of physics. Here, instead of using commercial equipment, they developed simple teaching tools that show physical phenomena in an exciting and attractive way, using quite common materials and daily goods. For example, the velocity of sound is measured by a pipe filled with a gas (air, $\mathrm{CO}_{2}$, helium, etc), setting a loudspeaker and two microphones in the pipe. Interference of sounds is demonstrated by two pipes with attached cone-shaped horns at one end, which collect a source sound in different phase, and merging them into one at the other end, which produce louder or quieter sound as a result of interference. Hitting or rubbing different length of aluminum rods aids students' understanding of longitudinal waves and transverse wave with the relationship between rod's length and pitch, as well as a pleasant experience with beautiful tones. These educational tools will be presented with videos taken with the Stray Cats group. 
2pEDa4. An acoustics education outreach program for young children. Ralph T. Muehleisen (Civil and Architectural Eng., Illinois Inst. of Technol., Chicago, IL 60616, muehleisen@iit.edu)

With limited time for science in the preschool and primary classroom, and limited science education of many preschool and primary school educators, the need for science professionals to engage in outreach education is ever increasing. Additionally, there is an increasing need for children to be aware of the problems of noise exposure, especially with regard personal sound systems. In this talk, a one-hour acoustics education outreach program developed for preschool and early primary school children is presented. In the program, children are involved in a discussion of the human auditory system and the dangers of noise exposure, engage in listening exercises using a keyboard synthesizer, construct a small musical instrument, and finally play the musical instrument with the rest of the class in a marching band.

2:25

2pEDa5. Let children experience speech science. Takayuki Arai (Dept. of Elec. and Electron. Eng., Sophia Univ., 7-1 Kioi-cho, Chiyoda-ku, Tokyo, 102-8554 Japan)

An education in acoustics is important for students of different ages: college, high school, middle school, and potentially even elementary school students, for whom such hands-on models are the most important. Because speech communication is a basic human activity, even for children, we expect that even the youngest students to be interested in speech science. With this in mind, we were motivated to develop intuitive and effective models for teaching speech science to all types of students. Our physical models of the human vocal tract [T. Arai, J. Phonetic Soc. Jpn., 5(2), 31-38 (2001)] have recently been installed in an exhibition in a museum in Japan. Children can watch, listen to, and touch the models. Our recent head-shaped and lung models [T. Arai, Acoust. Sci. Technol. 27(2), 111-113 (2006)] are also useful for educating students, because they are intuitive to produce a systematic and comprehensive education from the lungs to the head. Furthermore, we recently designed a sliding three-tube (S3T) model that has a simple mechanism for producing several different vowels [T. Arai, Acoust. Sci. Technol (to be published)]. This model is being used for an activity at a science workshop for children. [Work partially supported by JSPS.KAKENHI (17500603).]

\section{Contributed Papers}

\section{2:45}

2pEDa6. Development of educational software that supports study of sound environment for children. Hiroyuki Sakai (Ctr. for Promotion of Excellence in Higher Education, Kyoto Univ., Yoshida-Nihonmatsu, Sakyo, Kyoto 606-8501, Japan), Tadashi Shirakawa (NPO Onbunken, Tokyo, Japan), and Munetaka Tanaka (Sound Process Design, Inc., Tokyo, Japan)

A sound map, on which observed sounds are drawn as visual symbols, is well known as one of the nature games. Sound educational software, cultivating children's ecological view through their daily experiences focusing on sounds, was developed by using the sound map concept. Children record observed sounds through a sound-walk in outdoors into PC, and learn the sound environment through their collaborative work including classification, analysis, and group discussions in a workshop or a class. In a traditional approach on the field of educational technology, a lot of teaching materials introducing novel ICTs (information and communication technologies) are developed, but it is rather important to support usefulness for users (children and teachers), promote motivations for their learning, and support the smooth learning progressive process. In this presentation, the development concept of the software for the sound environment study based on socio-cultural approach is discussed, while an actual use example of this software is demonstrated.

\section{3:00}

2pEDa7. Stimulating children's interest in acoustics using models and toys. M. G. Prasad (Dept. of Mech. Eng., Stevens Inst. of Technol., Hoboken, NJ 07030)

Sound is an important means for sensory perception. Children, being naturally curious and sensitive, respond very well to sound. Simple acoustical toys attract their curiosity and attention. The use of simple sound- or music-producing tools draws their attention and then they ask questions.
In answering their questions simple principles of acoustics and scientific reasoning can be taught to them. Simple principles such as tone change due to change of volume in a resonator, the role of length in the sound produced by a tube, the role of damping in cutting down vibration and sound, etc. can be taught to the children. Also, the description of noise being unwanted sound can be demonstrated using simple mechanical models with comparison to desirable sound as music through instruments such as the flute. A model of the human ear can be used to describe the way the sound is perceived. The use of models and encouraging children to ask questions will further enhance their curiosity. The author will share his experiences gained through making presentations for elementary school children.

\section{3:15}

2pEDa8. Re-equipping the University of Sydney's acoustics laboratory for teaching after a fire. Ken Stewart and Densil Cabrera (Faculty of Architecture, Univ. of Sydney, NSW 2006, Australia)

For some decades, the University of Sydney has taught and conducted research in architectural and audio acoustics from the Faculty of Architecture's acoustics laboratory. In October 2005, a fire in the anechoic room destroyed the laboratory mainly through heat and smoke damage. Recovery has involved a reconsideration of the systems used for teaching and research, and this presentation outlines our approach to this. One consideration is that measurement systems should be relatively self-contained, so that setup time for measurements is minimized, and several setups can be used simultaneously for hands-on teaching. New equipment tends to be computer based, which has the advantages of much greater measurement power, automation, recording, and interfacing, but can be less intuitive for students to understand than individual analog components. This presentation compares the preexisting and renovated laboratory, especially in terms of teaching strategies in audio and acoustics using technical equipment. We present examples of previous and envisaged student projects to illustrate how such infrastructure can support learning. 
IAO NEEDLE/AKAKA FALLS ROOM, 3:45 TO 4:45 P.M.

\title{
Session 2pEDb
}

\section{Education in Acoustics: Take 5's}

Uwe J. Hansen, Cochair

64 Heritage Dr., Terre Haute, IN 47803

Kentaro Nakamura, Cochair

Tokyo Inst. of Technology, Precision and Intelligence Lab., 4259 Nagatsuta, Midori-ku, Yokohama, Kanagawa 226-8503, Japan

Chair's Introduction-3:45

Your favorite acoustics teaching devices. Bring a short demonstration, teaching device, or video, to share with your colleagues. A brief, descriptive handout is encouraged. Sign up at the door for a 5-minute slot. If you bring more than one demo, sign up for two non-consecutive slots.

\section{WEDNESDAY AFTERNOON, 29 NOVEMBER 2006}

MONARCH ROOM (ROYAL HAWAIIAN), 1:20 TO 4:15 P.M.

\section{Session 2pMU}

\section{Musical Acoustics: Comparing Asian and Western Instruments II}

\author{
Thomas D. Rossing, Cochair \\ Stanford Univ., CCRMA, Dept. of Music, Stanford, CA 94305 \\ Tomoyasu Taguti, Cochair \\ Konan Univ., 8-9-1 Okamoto, Higashi-ku, Kobe, 658-8501, Japan \\ Invited Papers
}

$1: 20$

2pMU1. Acoustical characteristics of Chinese stringed instruments and their Asian relatives. Akiko Odaka (Dept. of Musicology, Tokyo Natl. Univ. of Fine Arts and Music, 10-8 Uenokoen, Taito, Tokyo 110-8714, Japan) and Shigeru Yoshikawa (Kyushu Univ., Fukuoka 815-8540, Japan)

In Asia, there are many relative musical instruments that derive from the same origin or share a similar structure. In spite of their similarities, they show distinct differences from each other in terms of their musical usages, playing techniques, and acoustic effects. This varies with each musical genre, musical structure, social background, and acoustic taste swayed by those elements. People often add special devices to musical instrument or improve them so as to realize their acoustical ideas. For example, the shamisen and biwa, Japanese plucked lutes, have devices to allow strings to vibrate against the neck-wood, creating a reverberant high-frequency emphasis called sawari, which is of special value in each performance. A device that creates a similar effect, called jawari in Hindi, is also found in the sitar, a traditional stringed instrument of India. On the other hand, the Chinese sanxian, one of the relatives of the Japanese shamisen lacks this device. In this presentation, specific features of various musical instruments are introduced from both musicological and acoustical viewpoints. In particular, Chinese stringed instruments are focused on and compared with those of Japan, Korea, and India for investigation of their acoustical effects.

\section{1:40}

2pMU2. Vibro-acoustic measurement of several biwas. Tomoyasu Taguti (Konan Univ., 8-9-1 Okamoto, Higashinada, Kobe, Japan) and Kakuryo Tohnai (Nerima, Tokyo, Japan)

The biwa is a family of Japanese lutes that have been played for more than 1000 years. This paper reports some measurements on the vibro-acoustic characteristics of several biwas of different types. The main concern is the similarity and difference of the fukuju-to-table transfer mobilities among the individual biwas. Here, the fukuju is a kind of bridge whose shape and way of 
installation differ distictively from those common to the majority of other lute families found in many countries in the past as well as in the present time. The measurement revealed that in most cases the fukujus have two major vibration modes, to be labeled the "twist" and "flap" modes, with the corresponding resonance frequencies in the regions of about 0.9 to $1.2 \mathrm{kHz}$ and of 1.4 to $1.6 \mathrm{kHz}$, respectively, though the exact figures depend on the physical size and shape. Their resonance property takes an important roll to determine the fukuju-to-table transfer mobility as the coupled vibroacoustic system with the table. The fukuju-to-table mobilities were compared with the spectra of the corresponding plucked sounds.

2pMU3. The acoustics of the hammered dulcimer and similar instruments. David Peterson (Univ. of Central Arkansas, Conway, AR 72032)

The hammered dulcimer, an antecedent of the piano, apparently originated in the Middle East sometime around the 14th century. A typical instrument has 23 to 30 courses of strings, two to four strings per course, and is played with two wooden hammers. As in the yang qin and other Asian dulcimers, bridges separate some string courses into two notes. In American instruments the treble bridge creates a musical fifth, which facilitates the speedy playing of diatonic scales and also creates a strong harmonic structure to the tone. In response to player demand, modern builders have used improved or new materials (glues, woods, composites, plastic) and better design to create a lighter and less resonant instrument with a greater musical range. Dr. Peterson will demonstrate various playing styles.

2:20

2pMU4. Development of a carbon-fiber-reinforced composite for guitar soundboards: Investigation of bracing effect by experiment and simulation. Akira Okuda and Teruaki Ono (Faculty of Engineeing, Gifu Univ., Yanagido Gifu-City, Japan 501-1193, ono@cc.gifu-u.ac.jp)

A carbon-fiber-reinforced polyurethane foam composite whose physical and acoustic properties closely resemble those of wood for stringed-instrument soundboards was developed. A guitar with such a composite-made soundboard produced an almost identical tone to that produced with a wooden soundboard. The procurement of wood for soundboards, however, has not yet become very difficult. To accelerate the production and use of guitars with composite soundboards, it is necessary to create an attractive guitar that has characteristic and unique tones that cannot be realized on a wooden soundboard, making use of composite characteristics of design freedom. Wooden top boards, soundboards, have bracing that has traditionally and experientially been glued; however, the effects of bracing have never been clarified. In this study, the bracing effect on the frequency response and vibration mode in a guitar top board was investigated using experimentation and simulation. From the results obtained, a composite-made soundboard with no bracing, which produces more harmonics than a wooden one with bracing, was simulated as an example.

\section{2:40}

2pMU5. On the factors for detecting mistuned conditions and guiding their corrections for the Koto tuning. Tamaki Ando (Tokyo Natl. Univ. of Fine Arts and Music, 12-8 Ueno Park, Taitou-ku Tokyo, Japan 110-8714, an_tamaki@yahoo.co.jp)

By observations at the Koto tuning practice, it is realized that the bridge is moved in the period of about $400 \mathrm{~ms}$, which is too short to feel the sensation of beat. Then, two kinds of listening tests were planned. The test sounds composed of two Koto sounds which should be tuned in one octave interval were presented at the same time like a part of actual Koto tuning process. The lower tone was at $196.9 \mathrm{~Hz}$, the pitch of the higher one was varied in the range of 20 cents. Their length from the beginning was varied from 50 to $500 \mathrm{~ms}$. The first experiment is for the judgment of the right or wrong tuning (higher or lower). The second one is for the tone color impression using 13 pairs of adjectives. The results of these experiments show that reasonable pitch judgments can be done for the test sounds longer than $200 \mathrm{~ms}$ by well-experienced Koto players, and the test sounds are clearly distinguished from each other by their tone color difference, whereupon in the Koto tuning by expert players, the direction and degree of mistuning are found to be judged at the initial stage of Koto sounds by the tone color impression.

\section{3:00-3:15 Break}

\section{Contributed Papers}

3:15

2pMU6. Acoustics of the haegeum, a Korean string instrument. Junehee Yoo (Dept. of Phys. Education, Seoul Natl. Univ., Seoul 151-742, Republic of Korea, yoo@snu.ac.kr) and Thomas D. Rossing (Stanford Univ., Stanford, CA 94305)

The Korean haegeum, closely related to the Chinese erhu and Japanese kokyo, dates back to the Goryeo Dynasty. It is played with a horsehair bow that is inserted between two strings attached to a small barrel-shaped resonator. A bow made up of a bamboo stick with lengths of horsehair is held by the right hand of the performer. The two strings are generally tuned to $\mathrm{A} 3 \mathrm{~b}$ and $\mathrm{E} 4 \mathrm{~b}$ and the resonator is tuned to about $300 \mathrm{~Hz}$. The resonator has a paulownia wood plate at one end on which the bridge rests. By means of holographic interferometry, we compare modes of vibration in resonators of bamboo, ailanthus, birch, and ash.

\section{$3: 30$}

2pMU7. Inharmonic sounds of bowed strings in Western music and Beijing opera. Chen-Gia Tsai (Grad. Inst. of Musicology, Natl. Taiwan Univ., No. 1, Sec. 4, Roosevelt Rd., Taipei 106, Taiwan, tsaichengia@ntu.edu.tw)

Due to the nonlinear bow-string interaction, inharmonic sounds can be produced by bowed strings. Such sounds are characterized by subharmonics, sidebands, and noises. Bow location, bow speed, and bow pressure on the string have been found critical to producing inharmonic sounds on the violin or the cello. In the accompaniment music of Beijing opera singing, 
inharmonic sounds produced by the major bowed-string instrument (the jinghu) are sometimes called "blossoming tones." They are played with a special touch technique of the left hand, and a chaotic behavior of string vibration arises from a nonlinear boundary condition with unilateral con- straints. The spectra of blossoming tones can be viewed as high-pass noises, with the cutoff frequency of about $1.6 \mathrm{kHz}$. Blossoming tones are sophisticatedly used for emotional expressions in Beijing opera. Their harsh quality recalls some pathological human voices.

3:45-4:15

Mini-concert of Koto music by Tamaki Ando

WEDNESDAY AFTERNOON, 29 NOVEMBER 2006

WAIANAE ROOM, 1:00 TO 3:45 P.M.

\title{
Session 2pPA
}

\section{Physical Acoustics and Noise: Sonic Boom From Supersonic Aircraft and Projectiles II}

\author{
Victor W. Sparrow, Cochair \\ Pennsylvania State Univ., Graduate Program in Acoustics, 316B Leonhard Bldg., University Park, PA 16802 \\ Kazuyoshi Takayama, Cochair \\ Tohoku Univ., Biomedical Engineering Research Organization, 2-1-1 Katahira, Aoba, Sendai 980-8577, Japan
}

\section{Invited Papers}

1:00

2pPA1. Realism assessment of sonic boom simulators. Brenda M. Sullivan (NASA Langley Res. Ctr., M.S. 463, Hampton, VA 23681, brenda.m.sullivan@nasa.gov), Patricia Davies (Purdue Univ., West Lafayette, IN 47907-2031), Kathleen K. Hodgdon (Penn State Univ., State College, PA 16804-0030), Joseph Salamone (Gulfstream Aerosp. Corp., Savannah, GA 31407), and Anthony Pilon (Lockheed Martin Aeronautics Co., Palmdale, CA 93599)

The perceived realism of booms reproduced in three simulators, at NASA, Lockheed Martin, and Gulfstream Aerospace, was studied. The NASA and Lockheed facilities are loudspeaker-driven, airtight concrete structures that enable reproduction of the entire spectrum, including the very lowest frequencies. The Gulfstream facility is enclosed within a trailer and consists of a folded horn as well as loudspeakers. This creates the sonic boom waveform as a traveling wave but is unable to reproduce the lowest frequencies. In the tests, subjects experienced in listening critically to real sonic booms heard reproductions in the simulators of sonic booms recorded outdoors. Tests comparing the NASA/Gulfstream simulators and the Lockheed/Gulfstream simulators used the same outdoor sonic boom waveforms, though each simulator uses its own preprocessing to equalize the waveforms for presentation. The tests showed that the presence of ground reflections, very low frequencies, or recording hiss were not factors in realism ratings. However, the inclusion of some of the postboom noise heard after the boom itself was found to be extremely important for a realistic boom simulation.

$1: 20$

2pPA2. Sonic boom perception: Live versus simulator playback. Kathleen Hodgdon, Tom Gabrielson (Appl. Res. Lab., Penn State, University Park, PA 16802), Anthony Atchley (Penn State University Park, PA), Brenda Sullivan (NASA Langley Res. Ctr., Hampton, VA 23681), Joe Salamone, and Chris Babcock (Gulfstream Aerosp. Corp., Savannah, GA 31407)

A research team conducted a study at NASA Dryden Flight Research Center to compare the perception of real versus simulated booms. The field test included an assessment of noise measurement systems and a subjective jury trial on perception of sonic booms heard outdoors. Seventeen sonic booms were recorded under the flight path of four separate flights of three F-18s, using various microphones and recorders at multiple ground and ear level locations. Waveform analysis was conducted comparing the recordings as a function of location and measurement system. Eighteen subjects listened to live sonic booms, then rated the realism of the playback of a selection of those booms reproduced in the Gulfstream Supersonic Acoustic Signature Simulator II several hours later. The subjects also provided adjective descriptors of boom perceptions. The booms selected for the jury trial presented a variety of boom characteristics that included postboom noise. The subjects rated the playback as realistic, contributing to the validation of the simulator for use in future testing. Waveform comparisons and results from the field measurements and jury trial will be presented. [Work sponsored by FAA/NASA/TC PARTNER Center of Excellence.]

\section{$1: 40$}

2pPA3. Preliminary work on the development of a lexicon for supersonic aircraft sound. Andrew Marshall, Patricia Davies (Purdue Univ., 140 S. Intramural Dr., West Lafayette, IN 47907-2031, aimarsha@ purdue.edu), Brenda Sullivan (NASA Langley Res. Ctr., Hampton, VA 23681), and Anthony Pilon (Lockheed Martin Aeronautics Co., Palmdale, CA 93599)

A series of tests was conducted in three sonic boom simulators to test how expert subjects perceived the realism of the sound playback. Subjects heard sounds (recorded sonic booms) in two simulators and rated them on a realism scale. On completion of this realism test, subjects were asked to write down descriptions of the sounds and also of real booms (indoor and outdoor) that they had heard in the past. They then listened to 14 sounds and, after each sound, wrote down adjectives and phrases describing the sound 
characteristics. The 14 sounds were also analyzed and sound attribute metrics calculated for the main part of the boom as well as for the after-boom noise. The results of this analysis were compared to the words used to describe the sounds, and a preliminary set of words is proposed for inclusion in a lexicon for supersonic aircraft sound.

2:00

2pPA4. Human response to low-intensity sonic booms heard indoors and outdoors. Brenda M. Sullivan, Jacob Klos, Ralph D. Buehrle, David A. McCurdy (Structural Acoust. Branch, NASA Langley Res. Ctr., Hampton, VA 23681, brenda.m.sullivan@nasa.gov), and Edward A. Haering, Jr. (NASA Dryden Flight Res. Ctr., Edwards, CA 93523-0273)

A house on Edwards Air Force Base, CA, was exposed to low-intensity N-wave sonic booms during a 3-week test period in June 2006. The house was instrumented to measure the booms both inside and out. F-18 aircraft were flown to achieve a variety of boom overpressures from approximately 0.01 to $0.06 \mathrm{psf}$. During 4 test days, 77 test subjects heard the booms while seated inside and outside the house. Using the Magnitude Estimation methodology and artificial reference sounds, the subjects rated the annoyance of the booms. Since the same subjects heard similar booms both inside and outside the house, comparative ratings of indoor and outdoor annoyance were obtained. Preliminary results from this test will be presented.

2:20

2pPA5. Building structural acoustic response to aircraft sonic booms. Ralph D. Buehrle, Jacob Klos, Brenda M. Sullivan (Structural Acoust. Branch, NASA Langley Res. Ctr., Hampton, VA 23681), and Edward A. Haering, Jr. (NASA Dryden Flight Res. Ctr.)

As part of the NASA Low Boom/No Boom flight test project, a series of low-amplitude sonic-boom tests was completed over a 3-week period in June of 2006. This series of flight tests was designed to evaluate indoor/outdoor human subjective response, structural acoustic building response, and the effects of atmospheric turbulence for low-amplitude sonic booms characterized by overpressures in the nominal range of 0.1 to 0.6 pounds per square foot (psf). Low-amplitude sonic booms were generated by F-18 aircraft using dive trajectories to produce a range of overpressures at the Edwards Air Force Base housing area. In addition, straight and level supersonic flights were used to generate normal level (nominally $1.4 \mathrm{psf}$ ) sonic-boom overpressures at the housing area. This report will describe the structural acoustic building response measurements obtained during this flight test project. A single-family ranch-style home was instrumented with nearly 300 microphone and accelerometer sensors to determine the incident pressure and the building's structural acoustic response. Data were acquired for over 100 sonic-boom events. The test setup will be described and analysis of the measurements will be presented.

2:40

2pPA6. Modifications to the Gulfstream sonic boom simulator for the enhancement of low-boom simulations. Chris Babcock and Joe Salamone (Gulfstream Aerosp. Corp., P.O. Box 2206, Savannah, GA 31402)

A procedure is presented to enhance the simulation of measured low booms. Qualitative results show the SASSII, as originally designed, successfully reproduced the realism of full scale $\mathrm{N}$ waves. The simulator did not meet the realism level of the full-scale $\mathrm{N}$ waves when trying to reproduce the realism of lower amplitude sonic booms referred to as low booms. Data acquisition hardware was upgraded. Time alignment of each driver was performed with a different technique. Equalizer and amplifier programming was modified for each driver to provide a higher signal-to-noise ratio necessary to increase the realism of measured low booms in the simulator. A multipoint equalization was performed with a modified listening area. An iterative method rather than direct method was used to solve for the system of equations generated by the Weiner filtering scheme. The results are evaluated quantitatively by comparing the perceived loudness, A-weighted, and C-weighted sound-exposure levels of the full-fidelity waveform compared with the system output in the listening area.

3:00-3:15 Break

\section{Contributed Papers}

\section{3:15}

2pPA7. Prediction and measurement of sonic booms generated by the launch of a Minuteman II missile. Steven D. Pettyjohn (The Acoustcs \& Vib.. Group, 5700 Broadway, Sacramento, CA 95820-1852)

The Air Force wanted to launch a Minute II missile full range. Predictions were $15.9 \mathrm{psf}$ of over pressure at the sonic boom focus using similitude based on Saturn 5 data. Since this is at the threshold where single strength glass could break, a decision was made to predict over pressure using a NASA program. This program predicted a level of $16.1 \mathrm{psf}$ over pressure, just $1.3 \%$ greater than the original estimate. Confirmation was made by measuring the sonic boom from the launch out of Vandenberg Air Force Base. Seven small boats equipped with sound-activated tape recorders were placed along the flight path with some boats placed at the predicted focus point based on the weather forecast provided by the Air Force. Based on work by A.D. Pierce, two distinct N-wave types were expected. One would represent the path from where the missile first reached sonic speeds and would propagate along a fairly shallow path with respect to the earth's surface. The second wave type would arrive from a much higher elevation in the flight path and would propagate through many atmospheric layers. Weather forecasts were incorrect, but the results show the two shock wave types.

\section{3:30}

2pPA8. Modeling and measurements of nonlinear spherically divergent $N$ waves in air. Vera A. Khokhlova, Mikhail V. Averiyanov, Petr V. Yuldashev (Dept. of Acoust., Faculty of Phys., Moscow State Univ., Moscow 119992, Russia, vera@acs366.phys.msu.ru), Sebastien Ollivier, and Philippe Blanc-Benon (LMFA, Ecole Centrale de Lyon, 69134 Ecully Cedex, France)

Propagation of nonlinear $N$ pulses in relaxing medium was studied experimentally and numerically. An electric spark source was used in laboratory-scale experiments in ambient air to obtain spherically divergent shock pulses of $30-\mathrm{s}$ duration and $1000-\mathrm{Pa}$ peak pressure at $15-\mathrm{cm}$ distance from the source. Pressure waveforms were measured by the broad- 
band $\frac{1}{8}$-in. microphone at the distances varying from $15 \mathrm{~cm}$ to $2 \mathrm{~m}$ from the spark source. A numerical solution was obtained for an initial analytic $N$ waveform at the same distances based on the modified Burger's equation for nonlinear spherical waves in dissipative medium with relaxation. The duration of the initial $N$ wave was defined by matching the zero values of the corresponding analytic measured spectra; the amplitude was obtained by matching the experimental and theoretical nonlinear propaga- tion curves for the $N$-wave duration. The results of modeling were found to be in good agreement with the experimental data. A frequency response of the microphone was obtained at various distances as a ratio between the measured and modeled spectra and showed a good reproducibility. The relative effects of relaxation, nonlinearity, and microphone filtering on the $N$-waveform parameters are discussed. [Work is supported by RFBR and INTAS grants.]

\title{
Session 2pPP
}

\section{Psychological and Physiological Acoustics: Physiology, Psychoacoustics, Noise, Prostheses (Poster Session)}

\author{
Deborah A. Fantini, Cochair \\ Univ. of Essex, Dept. of Psychology, Wivenhoe Park, Colchester CO4 3SQ, U.K. \\ Kaoru Ashihara, Cochair \\ AIST, Tsukuba Central, 6 Tsukuba, 1-1-1 Higashi Ibaraki 305-8566, Japan
}

\section{Contributed Papers}

\begin{abstract}
All posters will be on display from 1:00 p.m. to 5:30 p.m. To allow contributors an opportunity to see other posters, contributors of odd-numbered papers will be at their posters from 1:00 p.m. to 3:15 p.m. and contributors of even-numbered papers will be at their posters from 3:15 p.m. to 5:30 p.m.
\end{abstract}

2pPP1. Umbo vibration in response to two-tone stimuli measured in human subjects. Diana Turcanu, Ernst Dalhoff, and Anthony W. Gummer (Univ. Tuebingen, HNO-Klinik, Elfriede-Aulhorn-Str. 5, 72076 Tuebingen, Germany)

The aim of these experiments was to noninvasively investigate cochlear mechanics near auditory threshold in human subjects by measuring the vibration response of the umbo. The vibration measurement setup consists of a custom-built laser Doppler vibrometer (LDV), employing a conventional heterodyne interferometer, for which all optical and electronic parts were tailored to the problem of measuring picometer-sized vibrations in the presence of large, extraneous movements in the order of $0.1 \mathrm{~mm}$ (e.g., heart beat, breathing, or swallowing). The cubic distortion product (DP) of the otoacoustic emissions (OAEs) in response to sound stimulation with two tones was measured as vibration on the umbo. For stimulus frequencies, $\mathrm{f} 1$ and $\mathrm{f} 2$, with $\mathrm{f} 2 / \mathrm{f} 1=1.2$ and $\mathrm{f} 2=4-9.5 \mathrm{kHz}$, and intensities $\mathrm{L} 1$ and $\mathrm{L} 2$, with $\mathrm{L} 1=0.4 \mathrm{~L} 2+39 \mathrm{~dB}$ and $\mathrm{L} 2=20-65 \mathrm{~dB}$ SPL, the vibration amplitudes were $1-8 \mathrm{pm}$ in subjects $(N=20)$ with hearing loss no more than $25 \mathrm{~dB}$ SPL. Application of this vibration technique to a recently proposed method [P. Boege and T. Janssen, J. Acoust. Soc. Am. 111, 1810-1818 (2002)] of objectively estimating hearing threshold by means of extrapolating DPOAE growth functions showed a standard deviation of the estimate of only $8.6 \mathrm{~dB}$.

2pPP2. Optical imaging of neural activity to vocalized sounds in the guinea-pig auditory cortex. Junsei Horikawa, Takashi Hatta, Shunji Sugimoto (Toyohashi Univ. of Technol., Toyohashi 441-8580, Japan), Yutaka Hosokawa (Ryukyu Univ., Okinawa 903-0215, Japan), and Michinori Kubota (Tokyo Med. \& Dental Univ., Tokyo 101-0062, Japan)

Spatiotemporal neural activity in response to vocalized sounds (calls) was investigated in the auditory cortex of anesthetized guinea pigs using an optical imaging technique with a voltage-sensitive dye (RH795). Guinea-pig calls were recorded digitally and presented to the ear from a loudspeaker located contralaterally to the recording cortex. Pure tone (PT), click (CLK), and white noise (WN) stimuli were used for comparison. The response in the primary (AI) and dorsocaudal (DC) fields of the auditory cortex to a PT appeared at the stimulus onset and in the corresponding frequency band, followed by nonsimultaneous lateral inhibition in the adjacent frequency bands. The responses to CLK and WN stimuli appeared in a wide area of the fields corresponding to the wide frequency components of the stimuli. The response to calls changed spatially and temporally because the components' frequencies changed over time. Strong responses occurred in the frequency bands corresponding to prominent instantaneous frequency components. These results suggest that activity to calls in AI and DC fields is formed by spatiotemporal excitatory and inhibitory interactions between the frequency bands responding to instantaneous frequency components in the calls. [Work supported in part by the 21st century COE program "Intelligent Human Sensing" from the MESSC, Japan.]

2pPP3. Extraction of sounds' time-frequency features in the auditory cortex. Shunji Sugimoto, Yuuta Suzuki, Hiroyuki Tanaka (Toyohashi Univ. of Technol., Toyohashi 441-8580, Japan, sugimoto@tutkie.tut.ac.jp), Michinori Kubota (Tokyo Med. \& Dent. Univ., Tokyo 101-0062, Japan), and Junsei Horikawa (Toyohashi Univ. of Technol., Toyohashi 441-8580, Japan)

Although "time-frequency feature extraction" is a fundamental cue that is used to recognize speech sounds, its physiological mechanisms remain largely unknown. This research is aimed at understanding what time-frequency features of sounds are extracted by auditory cortical neurons. We investigated spatiotemporal response patterns in the guinea-pig auditory cortex, using an optical recording method (MiCAM Ultima; Brain Vision) with a voltage-sensitive dye (RH795). Experiments were performed under anesthesia (ketamine, $100 \mathrm{mg} / \mathrm{kg}$, and xylazine, $25 \mathrm{mg}$ / $\mathrm{kg}$ ). Pure tones (PTs), frequency-modulated (FM) sounds with various modulation rates, and their combinations were presented to the animals. Cortical activation was induced at the onset and offset of PTs along the 
isofrequency contours. The onset response was followed by strong inhibition. In contrast, FM sounds evoked an additional activation moving across the isofrequency contours in the cortex, with locations corresponding to the instantaneous frequency input of the FM sweep. Such a response became more evident when the FM sweep rate increased. Based on these results, we discuss physiological mechanisms by which sounds with a rapid frequency modulation lead to "time-frequency feature extraction" in cortical neurons. [Work supported in part by the 21 st century COE program "Intelligent Human Sensing” from the MESSC, Japan.]

2pPP4. An attempt to measure the hearing thresholds for highfrequency sounds by using the auditory brain-stem response. Kazumasa Hosokawa (Musashi Inst. of Technol., 1-28-1 Tamatsutsumi, Setagaya-ku, Tokyo, 105-8557 Japan), Kaoru Ashihara (AIST, Tsukuba, Ibaraki, 305-8566 Japan), and Shogo Kiryu (Musashi Inst. of Technol., Setagaya-ku, Tokyo, 105-8557 Japan)

Recently, devices using high-power ultrasounds are increasing. However, influences of high level ultrasounds on human are not fully understood. Psychophysical studies show that the hearing thresholds exceed 80 $\mathrm{dB}$ SPL at a frequency above $20 \mathrm{kHz}$. It is reported that high frequency sounds above $20 \mathrm{kHz}$ affect activities in the human nervous system. In order to examine whether the peripheral auditory system can be activated by high-frequency sounds, an attempt is made to measure the auditory brainstem response (ABR) for the frequency range between 4 and $20 \mathrm{kHz}$. In preliminary experiments, the ABR was measured for a tone pip with the center frequency of $14 \mathrm{kHz}$. However, the ABR was not clearly observed above $16 \mathrm{kHz}$. The thresholds determined by the ABR were approximately $40 \mathrm{~dB}$ larger than the psychophysical thresholds. The ABR was evoked by low-frequency components of the tone pip at the high presentation levels.

2pPP5. Distortion product otoacoustic emission fine structure as an early hearing loss predictor. Karen Reuter and Dorte Hammershøi (Dept. of Acoust., Aalborg Univ., Fredrik Bajers Vej 7, B5, 9220 Aalborg Øst, Denmark, kr@acoustics.aau.dk)

Otoacoustic emissions (OAEs) are a promising method to monitor early noise-induced hearing losses. When distortion product otoacoustic emissions (DPOAEs) are obtained with a high-frequency resolution, a ripple structure across frequency can be seen, called DPOAE fine structure. In this study DPOAE fine structures are obtained from 74 normalhearing humans using primary levels of L1/L2 $=65 / 45 \mathrm{~dB}$. The subjects belong to groups with different ages and exposure histories. A classification algorithm is developed, which quantifies the fine structure by the parameter's ripple place, ripple width, ripple height, and ripple prevalence. Temporary changes of the DPOAE fine structure are analyzed by measuring DPOAE both before and after exposing some of the subjects to an intense sound. The characteristic patterns of fine structure can be found in the DPOAE of all subjects, though they are individual and vary from subject to subject within groups. The results do not indicate that the DPOAE fine structure alters with the state of hearing, as it is suggested in the literature. The data analysis is still in process at this stage.

2pPP6. The behavior of the distortion product otoacoustic emissions in response to contralateral stimulation by stationary and nonstationary signals. Olimpia Szczepaniak (Div. of Rm. Acoust. and Psychoacoustics, Adam Mickiewicz Univ., Umultowska 85, 61-614 Poznan, Poland, oli0809@interia.pl)

The main aim of the study was to determine the DPOAE of the cochlea in the case of contralateral stimulation by stationary and nonstationary signals. The analysis was performed for $3 f_{1}-2 f_{2}, 2 f_{1}-f_{2}, 2 f_{2}-f_{1}$ intermodulation distortion products on a group of 20 normal hearing subjects $(\mathrm{NH})$ and ten patients with low UCL (uncomfortable loudness level). The optimized primary tone level setting $\left(L_{1}=0.4 L_{2}+39 \mathrm{~dB}\right)$ was used. Each $\mathrm{NH}$ subject was characterized by the I/O function of otoacoustic emission (40-90 dB SPL) and dp-grams for the three levels of two-tone $(85 / 75,75 / 65$, and 65/55 dB SPL) for frequencies between 1 and $12 \mathrm{kHz}$. Parameters for subjects with UCL were individually selected. The suppression of otoacoustic distortion products and the intergroup correlation of the slopes of I/O function with various contralateral stimulators were discussed. Results obtained from the experiment were compared between the two groups studied.

2pPP7. A functional MRI study on auditory feedback control of pitch. Akira Toyomura (RIES, Hokkaido Univ., Sapporo 060-0812 Japan /JST, toyomu@es.hokudai.ac.jp), Sachiko Koyama, Tamaki Miyamoto, Atsushi Terao (Hokkaido Univ., Sapporo 060-0812, Japan), Takashi Omori (Tamagawa Univ., Tamagawa 194-8610, Japan), Harumitsu Murohashi, and Shinya Kuriki (Hokkaido Univ., Sapporo 060-0812, Japan)

Perception of one's own speech plays an important role in fluent speech production. In this study, we conducted a functional magnetic resonance imaging (fMRI) experiment to delineate the neural mechanism for auditory feedback control of pitch, using a transformed auditory feedback (TAF) method. Subjects ( $n=12$, right handed) were required to vocalize /a/ for $5 \mathrm{~s}$ while hearing their voice through a headphone. In the TAF condition, the feedback voice pitch was shifted randomly up or down from the original pitch two or three times. The subjects were required to hold the pitch of the feedback voice constant by changing the pitch of the original voice. In the non-TAF condition, the pitch of feedback voice was not modulated and the subjects merely vocalized /a/ continuously. A contrast between TAF and non-TAF conditions showed some significantly greater activations in the TAF condition $(p<0.05$ corrected). In the right hemisphere, the supramarginal gyrus, the prefrontal area, the anterior insula, and the superior temporal gyrus showed greater activation. In the left hemisphere, only the premotor area showed greater activation. This result suggests that auditory feedback of pitch is controlled mainly by the right hemispheric network. [Work supported by JST.]

2pPP8. Mechanisms of bone-conducted ultrasonic perception assessed by electrophysiological measurements in human. Seiji Nakagawa (Natl. Inst. of Adv. Industrial Sci. and Technol. (AIST), 1-8-31 Midorigaoka, Ikeda, Osaka 563-8577, Japan, s-nakagawa@aist.go.jp) and Aya Nakagawa (Natl. Inst. of Adv. Industrial Sci. and Technol. (AIST)/ Suita Municipal Hospital, Osaka, Japan)

Several studies have reported that bone-conducted ultrasound (BCU) is perceived even by the profoundly sensorineural deaf; however, the mechanisms involved remain unclear. We previously reported some unique characteristics of BCU perception: (1) the pitch of BCU is about ten-odd $\mathrm{kHz}$ and is independent of its frequency, (2) the dynamic range of $\mathrm{BCU}$ is narrow, less than $20 \mathrm{~dB}$, and (3) BCU mask $10-15-\mathrm{kHz}$ air-conducted sounds. In this study, cortical magnetic field $(\mathrm{N} 1 \mathrm{~m})$, middle latency (MLRs) and auditory brainstem responses (ABRs) and electrocochleogram (EcochG) evoked by BCU in human were recorded to clarify the neural pathway for BCU. Substantial MLRs and ABRs (wave-I-V) were evoked by BCU as well as by air-conducted sound. Although a clear N1m response was elicited by $\mathrm{BCU}$ and its equivalent current dipoles (ECDs) were estimated in the auditory cortices, ECD locations for BCU did not follow the tonotopic organization at the cortical level. Both wave I of $\mathrm{ABRs}$ and $\mathrm{AP}$ of EcochG were observed clearly, indicating that the auditory nerve was substantially activated. These results suggest that BCU goes through the normal auditory pathway-there are no special organs for BCU perception; however, unique processes may exist in the cochlea. 
2pPP9. Interaction between the tonotopic and periodic information in recognition of "melodic" contours. Toshie Matsui, Chihiro Takeshima (Kyoto City Univ. of Arts, Grad. School of Music, 13-6, Kutsukake-cho, Oe, Nishikyo-ku, Kyoto 610-1197, Japan, m04905@kcua.ac.jp), and Minoru Tsuzaki (Kyoto City Univ. of Arts, Kyoto 610-1197, Japan)

It is known that the tonotopic and periodic aspects of sounds are separated in the auditory sense. To investigate the manner of representing these two aspects, a psychological experiment was designed. The stimuli are sequences of vowels, each of which changes its fundamental frequency and "size," i.e., the centroid of its formants, using a STRAIGHT vocoder. Participants are asked to respond if the transient patterns of the stimuli are "clockwise" or "counterclockwise" in a two-dimensional space, one of whose coordinates is the periodicity and the other of which is the size. Results indicate that the judgment was not so easy as in the case in which one would judge the transition patterns of points in a two-dimensional visual space. This inference implies that the two attributes might be represented as values on two completely independent scales, but not like the values in two coordinates of a single point.

2pPP10. A preliminary study of construction of a musical mood scale. Junko Matsumoto (Nagano College of Nursing, 1694, Akaho, Komagane, Nagano, 399-4117, Japan, matsumoto@nagano-nurs.ac.jp)

In this study to construct a mood scale that was specific to listening to music, appropriate items were selected preliminarily. Three hundred three university students were presented 70 adjectives by a questionnaire and asked to rate how frequent they feel each mood after listening to music in their daily lives on a four-point scale. A factor analysis were completed on the mood items and yielded five factors indicating suffering, comfortableness, solemnness and aggression, calmness, and sadness, respectively. Items with high loading on these factors were selected to construct a musical mood scale and any of them were modified because they might have an obscure meaning.

2pPP11. Sensitivity analysis to evaluate the benefits and risks of using prior information in adaptive psychometric procedures. Jeremiah Remus and Leslie Collins (Dept. of Elec. and Comput. Eng., Duke Univ., Box 90291, Durham, NC 27708)

Numerous adaptive psychometric procedures have been developed based on a Bayesian framework. In these procedures, a key component for estimating the targeted parameter and determining the next stimulus value is the parameter probability distribution. The presence of a parameter probability distribution introduces the opportunity to include prior information via initialization of the parameter probabilities. However, differences in the techniques for parameter estimation and stimulus selection may determine how various psychometric procedures benefit from accurate prior information or recover from inaccurate prior information. In this study, several adaptive psychometric procedures using a Bayesian framework were investigated through simulation of a psychophysical task. The simulations included prior information with varying degrees of accuracy and varying levels of initial confidence. Results suggest that the benefits of prior information are dependent on the specific psychometric procedure and the inclusion of prior information may influence the final choice of an appropriate psychometric procedure.

2pPP12. Observed variability in $d^{\prime}$ for yes-no and two-interval forced choice procedures. Walt Jesteadt, Lori Leibold, Samar Khaddam, Jessica Messersmith, and Melissa Krivohlavek (Boys Town Natl. Res. Hospital, 555 N. 30th St., Omaha, NE 68131)

The sampling distribution of $d^{\prime}$ estimates can be determined from knowledge of the true $d^{\prime}$ and the number of trials per estimate [J. Miller, Percept. Psychophys. 58, 65-72 (1996)]. Five subjects were tested in a fixed-level intensity discrimination task to obtain estimates of the observed variance of $d^{\prime}$ for both the yes-no and two-interval forced-choice
(2IFC) procedures over a wide range of $d^{\prime}$ values. The stimuli were $2-\mathrm{kHz}$ sinusoids, $300 \mathrm{~ms}$ in duration with 10 -ms ramps, with differences in intensity ranging from 0.1 to $4.9 \mathrm{~dB}$, centered on $70 \mathrm{~dB}$ SPL. An estimate of $d^{\prime}$ was computed for each of ten 100-trial blocks at each intensity difference for each procedure. Psychometric functions, based on 14000 trials per function, were well fitted using intensity differences expressed in units of $\mathbf{\Delta} \mathbf{L}$ and were steeper for 2IFC than for yes-no. The variance of $d^{\prime}$ estimates exceeded the variance of the sampling distribution by a factor of 2.1 for yes-no and a factor of 3.1 for 2IFC, with learning effects contributing to the additional variance in some subjects. For a given value of $d^{\prime}$, the variance of $d^{\prime}$ estimates was approximately equal in yes-no and 2IFC. [Work supported by NIH/NIDCD.]

2pPP13. Neural correlates of psychometric functions for auditory detection. Huanping Dai (Div. of Commun. Disord., College of Health Sci., Univ. of Wyoming, Laramie, WY 82071)

The psychometric function in a 2IFC task, which describes the relation between the percentage of correct responses $(\mathrm{pc})$ and the signal strength $(x)$, can be expressed using the Gaussian probability function $(\phi)$ with a threshold $(\alpha)$ and a slope $(\beta)$ parameter, $p c=\phi\left[(x / \alpha)^{\beta} / 2^{1 / 2}\right]$. For auditory detection, the psychometric functions exhibited a wide range of slopes in different conditions, such as tone in noise $[\beta \approx 1, \mathrm{D}$. M. Green, M. J. McKey, and J. C. R. Licklider, J. Acoust. Soc. Am. 31, 1446-1452 (1959) $]$ and tone in quiet $[\beta \approx 2$, C. S. Watson, J. R. Franks, and D. C. Hood, J. Acoust. Soc. Am. 52, 633-643 (1972)] by listeners with acoustic hearing, and detection by listeners with electrical hearing $\left[\beta_{\max } \approx 10\right.$, G. S. Donaldson, N. F. Viemeister, and D. A. Nelson, J. Acoust. Soc. Am. 101, 3706-3721 (1997)]. To understand the mechanisms underlying the change in the slope of the psychometric function, the performance of a neural detector engaged in the same tasks as the listeners was analyzed, and the neural correlates of the psychometric function, the neurometric function, was derived. Under the assumption that the detection was based on spike counts of the neuron(s) tuned to the signal frequency, the derived neurometric function showed slope values in good agreement with those obtained from the listeners.

2pPP14. Perceptual organization in loudness enhancement and decrement. Deborah A. Fantini (Dept. of Psych., Univ. of Essex, Wivenhoe Park, Colchester, CO4 3SQ, England) and Christopher J. Plack (Lancaster Univ., Lancaster, LA1 4YF, England)

Loudness enhancement is an increase in the loudness of a target tone that occurs when a more intense inducer tone either precedes or follows the target. Loudness decrement is a similar effect in the opposite direction. The present work investigates the role of perceptual organization in this paradigm by employing a capture tone presented simultaneously with the inducer. In a pair of experiments, the inducer, target, and comparison tone were always $1 \mathrm{kHz}$. The capture tone, when present, was either 2.0 or 0.5 $\mathrm{kHz}$ (an octave above or below the target). A 10-ms inducer tone immediately preceded a $10-\mathrm{ms}$ target, with a 10 -ms comparison tone presented $850 \mathrm{~ms}$ later. Listeners were instructed to ignore the inducer and to judge whether the target or comparison tone was louder. Equal-loudness matches were estimated using two randomly interleaved adaptive tracks, one converging on the just-louder threshold and the other on the just-quieter threshold. Results from both enhancement and decrement experiments indicate that the addition of a capture tone significantly reduces the magnitude of the effect, however it does not eliminate the effect completely. These findings indicate that perceptual organization does play a role in the effects of an inducer tone on loudness judgments. 
2pPP15. Determining the area of the sweet spot in a surround loudspeaker setup for various microphone techniques. Nils Peters (Music Technol. Area, Schulich School of Music, McGill Univ., 555 Sherbrooke St. West, Montreal, PQ H3A 1E3, Canada, nils.peters@mcgill.ca) and Jonas Braasch (Rensselaer Polytechnic Inst., Troy, NY 12180-3590)

Several types of microphone techniques exist to record music performances for surround-sound reproduction. Variations between different techniques are found in the distance and angle between the microphones, and the choice of directivity patterns. All the arrays are targeted to produce an accurate spatial impression at the sweet spot. The aim of this investigation is to determine how different microphone techniques affect the size of the sweet spot, the area in which the spatial cues are reproduced with sufficient accuracy. In particular, the common belief that spaced techniques lead to larger sweet-spot areas than coincidence and nearcoincident techniques is investigated. For this purpose, impulse responses (IR) of different microphone arrays are measured in a concert hall. Afterwards, test sounds are convolved with the measured IRs and presented through a surround loudspeaker setup in a control room. A dummy head is used to record the reproduced sound fields at different positions inside the listening area. In a psychoacoustic experiment, listeners are asked to rate the different recordings according to the spatial impression provided by different recording techniques. The results of the listening test will be presented and compared to signal analyzes of a binaural model.

2pPP16. Perceptual dimensions of impulsive-source active sonar echoes. Jason E. Summers, Derek Brock, Brian McClimens, Charles F. Gaumond, and Ralph N. Baer (Naval Res. Lab., Washington, DC 20375-5350)

Recent findings [J. Pitton et al., J. Acoust. Soc. Am. 119, 3395(A) (2006)] have joined anecdotal evidence to suggest that human listeners are able to discriminate target from clutter in cases for which automatic classifiers fail. To uncover the dimensions of the perceptual space in which listeners perform classification, a multidimensional scaling (MDS) experiment was performed. Subjects rated the aural similarity between ordered pairs of stimuli drawn from a set of 100 operationally measured sonar signals, comprising 50 target echoes and 50 false-alarm clutter echoes. Experimental controls were employed to evaluate consistency in judgments within and between subjects. To ensure that dimensions were discovered rather than imposed [Allen and Scollie, J. Acoust. Soc. Am. 112, 211-218 (2002)], subjects were neither trained in classification nor made aware of the underlying two-class structure of the signal set. While training improves classification performance, prior work suggests that both expert and nave listeners use the same perceptual space [R. Mackie et al., ONR Tech. Rep. 2723-1, NR 197-060/4-18-79 (1981)]. Results of the MDS study are presented, including an evaluation of the number of perceptual dimensions and their interpretation in terms of perceptual and physical attributes of the signals. [Work supported by ONR.]

2pPP17. Remote psychoacoustic testing using the Internet. Alastair C. Disley, David M. Howard, and Andrew D. Hunt (Dept. of Electron., Univ. of York, Heslington, York, YO10 5DD, UK)

A number of recent listening tests by the authors and others have begun to use the Internet as a medium for remotely conducting psychoacoustic listening tests. While this offers a solution to the problem of gathering enough highly trained listeners in one geographic location, it results in a reduction in control of factors such as the listener's immediate environment. A recent experiment by the authors [A. C. Disley, et al., "Spectral correlations of timbral adjectives used by musicians," J. Acoust. Soc. Am. 119, 3333 abstract only (2006)] offered the opportunity to examine and quantify the effect of remote testing via the Internet. Twenty four listeners took an Internet-based listening test on ten separate occasions, alternating between a controlled environment and an uncontrolled environment. Listeners were given instructions about their uncontrolled listening environments, and data subsequently collected demonstrate good but not universal compliance with those instructions. Comparison of experimental results between the different test environments shows very little difference, with a slight reduction in the controlled environment to the listeners' self-assessed confidence in their judgments. The techniques, advantages, and disadvantages of the Internet as a medium for remote psychoacoustic testing are discussed.

2pPP18. +Gz acceleration modifies low-frequency perception. Patrick M. B. Sandor, Lionel Pellieux (IMASSA-SC, F91223, Bretigny-sur-Orge, France), and Christophe Dehlinger (LEGTP Stanislas, F67160 Wissembourg, France)

Hypergravity is associated with maneuvering acceleration $(+\mathrm{Gz})$ in fighter aircraft and can be simulated in a human centrifuge, two noisy environments where classical audiometric data in silence cannot be obtained. Yet under $+4-\mathrm{Gz}$ acceleration, with masking noise, the hearing threshold just superior to the masking noise upper limit frequency was reported to be slightly reduced and therefore hearing paradoxically enhanced [Sandor et al., Aviat. Space Environ. Med. 75, 9525 (2004)]. It was analyzed as an indirect evidence of attenuation of low-frequency perception in relation with the shape of the masking noise and the alteration of associated upwards spread of masking effect. A pressure modification in cochlea was supposed to occur under $+\mathrm{Gz}$, due to transmission of intracranial pressure through the cochlear duct, as observed in a clinical case [Portier et al., Ann. Otol. Rhinol. Laryngol. 111, 817-820 (2002)]. We developed a finite element model of cochlear mechanics revealing a shape modification of the apical side of cochlea under the pressure modification compatible with $+4-\mathrm{Gz}$ acceleration. Manoussaki and colleagues [Phys. Rev. Lett. 96(8), 088701 (2006)] emphasize the role of cochlea curvature in low frequency perception. Our experimental and computational results reinforce this role of cochlea curvature in low frequency perception.

2pPP19. Predictors of auditory performance in hearing-aid users: The role of cognitive function and auditory lifestyle. Martin D. Vestergaard $^{\text {a) }}$ (Ørsted-DTU Acoust. Technol., Tech. Univ. of Denmark, DK-2800 Lyngby, and Res. Ctr. Eriksholm, Kongevejen 243, DK-3070 Snekkersten)

Within clinical audiology, it is often observed that patients who are expected to perform the same differ in auditory performance. Hearing-aid users may be dissatisfied with their instruments while they score satisfactorily in objective tests, or they may be satisfied with their instruments while no objective benefit can be measured. It has been suggested that lack of agreement between various hearing-aid outcome components can be explained by individual differences in cognitive function and auditory lifestyle. We measured speech identification, self-report outcome, spectral and temporal resolution of hearing, cognitive skills, and auditory lifestyle in 25 new hearing-aid users. The purpose was to assess the predictive power of the nonauditory measures while looking at the relationships between measures from various auditory-performance domains. The results showed that only moderate correlation exists between objective and subjective hearing-aid outcome. Different self-report outcome measures showed a different amount of correlation with objective auditory performance. Cognitive skills were found to play a role in explaining speech performance and spectral and temporal abilities, and auditory lifestyle was correlated with self-report outcome. However, overall the predictive leverage of the various measures was moderate, with single predictors explaining only up to 19 percent of the variance in the auditory-performance measures. ${ }^{\text {a) }}$ Now at CNBH, Department of Physiology, Development and Neuroscience, University of Cambridge, UK. 
2pPP20. Perception and production of virtual pitch by absolute-pitch musicians. I-Hui Hsieh and Kourosh Saberi (Dept. of Cognit. Sci., Univ. of California at Irvine., Irvine, CA 92697, ihsieh@uci.edu)

The ability of absolute pitch (AP) and control (non-AP) musicians to identify and produce musical pitch from long-term memory was investigated in three experiments. In experiment 1 , subjects identified the pitch of either a missing-fundamental complex or a highpass-filtered piano note. Unlike control subjects, AP subjects were highly accurate (i85\%) in identifying virtual pitch even when cues were restricted to the 4th and 5th harmonics. In a second experiment, subjects produced, within $3 \mathrm{~s}$, the pitch of a randomly selected musical note without an external acoustic reference through either (1) adjusting a octave-range slider or (2) voicing/ singing the target note. AP subjects, but not controls, were highly accurate (0.3 semitone) in the adjustment task. However, both AP and non-AP subjects were extremely accurate (i.e., $\mathrm{SD}=0.97,0.90$ semitone error, respectively) in voicing the required pitches as determined from FFTs of the recorded voiced notes. In experiment 3 , subjects identified pitch of solfeggio syllables at mismatched note frequencies, created by altering the sampling rate of digitally recorded voiced labels. Identification accuracy by AP subjects declined to $65 \%$, supporting a cognitive/linguistic interference model. Implications of these findings for models of absolute pitch encoding will be discussed. [Work supported by NSF.]

2pPP21. Pitch estimation of bone-conducted ultrasound. Kazuhito Ito and Seiji Nakagawa (Natl. Inst. of Adv. Industrial Sci. and Technol., 1-8-31 Midorigaoka, Ikeda, Osaka 563-8577, Japan, keith.ito@aist.go.jp)

Although ultrasound is perceptible through bone conduction, its perception mechanism has been clarified only slightly. The subjective pitch of bone-conducted ultrasound (BCU) sounds like a tone or harmonic sound of ten-odd kilohertz, independent of its own frequency. It has been difficult to determine the frequency of the pitch precisely because the pitch varies among listeners and with the position and strength of BCU stimulation, moreover; $\mathrm{BCU}$ hearing is not masked well by air-conducted (AC) band-pass noise. In this study, the BCU pitch-perception mechanism was investigated using beats with $\mathrm{AC}$ tones. The $\mathrm{AC}$ tones of $10-17 \mathrm{kHz}$, for which the presence of the pitch of $\mathrm{BCU}$ had been expected, were presented sequentially together with $\mathrm{BCU}(30 \mathrm{kHz})$ stimulation. Listeners were asked whether or not they were able to hear beats. For comparison, bone-conducted (BC) harmonic sounds of audible frequency components (e.g., 11-16 kHz, 1-kHz step) were also investigated. Results showed that no beat was heard between the $\mathrm{AC}$ tones and the $\mathrm{BCU}$, whereas beats between the $\mathrm{AC}$ tones and the $\mathrm{BC}$ harmonic sounds were heard, suggesting the possibility that the pitch-perception mechanism of $\mathrm{BCU}$ is different from those of $\mathrm{AC}$ and $\mathrm{BC}$ sounds in this audible frequency area.

2pPP22. Off-frequency listening in a pitch matching experiment. Veronica Eckstein and Bruce G. Berg (Dept. of Cognit. Sci., Univ. of California Irvine, 3151 Social Sci. Plaza, Irvine, CA 92697)

A pitch-matching paradigm was used to investigate the pitch of threetone complexes. For different conditions, the central tone was 200, 1000, or $2000 \mathrm{~Hz}$ with the side tones spaced by either 30 or $45 \mathrm{~Hz}$. The tones were of equal intensity or a signal was added in phase to the central tone with a level of 5,0 , or $5 \mathrm{~dB}$ relative to the side tones. Listeners adjusted the frequency of a pure tone to match the pitch of the complex. According to a model known as the envelope weighted average of the instantaneous frequency (EWAIF) [L. Feth, Percept. Psychopys. 15, 375-378 (1974)], the pitch should be approximately equal to the center frequency for all conditions. Contrary to this prediction, increasing the bandwidth or decreasing the signal level systematically lowered the pitch. However, the results are consistent with EWAIF calculations if the stimuli are first passed through the skirt of an auditory filter. This method may provide an alternative technique for estimating the filtering properties of the auditory system. [Work supported by Center for Hearing Research, UCI.]
2pPP23. Sample discrimination of frequency differences with ipsilateral and contralateral distracters. Donna L. Neff, Walt Jesteadt, and Lori J. Leibold (Boys Town Natl. Res. Hospital, 555 N 30th St., Omaha, NE 68131)

This experiment examined the effect of ipsilateral and contralateral tonal distracters on sample discrimination of frequency differences. In the 2AFC baseline task, four listeners indicated which of two intervals, each containing a two-tone target complex, was higher in pitch. The pairs of target tones were drawn from Gaussian frequency distributions centered at low-, middle-, and high-frequency regions. Two distracter tones were then added, one from each of the two nontarget frequency regions (e.g., low targets, middle and high distracters). Distracter tones were either randomly drawn from a Gaussian frequency distribution or fixed in frequency at the mean of the distribution. Across conditions, targets were in the left or right ears, with distracters in the ipsilateral or contralateral ear. There was little effect of target ear or effect of adding fixed-frequency distracters in either ear. Effects of random-frequency distracters varied across subjects and frequency regions, with both ipsilateral and contralateral distracters showing large effects for some subjects and conditions. Distracters were least effective with middle-frequency targets. The ability to use the ear as a channel appears to depend on both the relative variability and relative frequency regions of the targets compared to the distracters. [Work supported by NIDCD.]

2pPP24. Dynamic center of gravity effects: Matching virtual frequency glides. Evelyn M. Hoglund, Lawrence L. Feth (Dept. of Speech and Hearing Sci., The Ohio State Univ., Columbus, OH 43210), and Ashok K. Krishnamurthy (The Ohio State Univ., Columbus, OH 43210)

Lublinskaja (1996) demonstrated that changing the amplitudes of tworesonance Russian vowels over time leads listeners to hear a glidelike transition that follows the dynamic center of gravity (COG). Anantharaman (1998) investigated COG transitions for sinusoidal signals by asking subjects to match frequency-modulated (FM) to virtual frequency (VF) glides. The amplitudes of two tones were simultaneously modulated to produce a percept of a glide with rising pitch, similar to that of an FM sweep, although the frequencies remained fixed. However, listeners might perform Anantharaman's task by simply attending to the location of one of the anchor tones without perceiving the virtual glide, as the frequencies of the two component tones were changed to alter the frequency span of the VF glide. This study modified that work by fixing the anchor frequencies, but confirmed the potential for procedural artifacts. Two additional experiments eliminated FM signals altogether and asked listeners to match VF glides to VF glides. Even with anchor frequency roving, subjects were able to match the VF glide trajectories for frequency separations up to 6 to 8 ERB. Results were compared with predictions from the perceptual spectral centroid model. [Research supported by a grant from NIH/NIDCD R01-DC006879.]

2pPP25. Spectrotemporal structure influences spatial release from informational masking in reverberant settings. Erol Ozmeral, Priyanka Zutshi, Virginia Best, and Barbara Shinn-Cunningham (Dept. of Cognit. and Neural Systems, Boston Univ., 677 Beacon St., Boston, MA 02215, ozmeral@cns.bu.edu)

Spatial separation between target and masking sources improves target identification due to both changes in energy (acoustic benefit) and the ability to focus attention on the target (spatial benefit). Studies of speech intelligibility show that while acoustic benefits are reduced by reverberation, spatial benefits remain robust [Kidd et al., Acustica 114, 526-536 (2005)]. The current study extends a previous study of nonspeech communication calls [birdsong; Best et al., J. Acoust. Soc. Am 118, 3766-3773 (2005)] to explore whether, like speech, the spatial benefit for these signals is robust in reverberation. It was found, however, that reverberation decreased both acoustic and spatial benefits for birdsong identification. To examine whether the decrease in the spatial benefit was due to spectrotem- 
poral differences between speech and birdsong, songs were slowed down to half the original rate (making them more similar to speech). For these songs, the spatial benefit was preserved in reverberation. These results suggest that spatial benefits in reverberation are less effective for rapidly fluctuating signals, presumably because reverberation smears their temporal structure, impairing segregation of competing syllables. Thus, the relatively slow fluctuations in speech may be important for its resilience to interference from other talkers in reverberant environments. [Work support by Grants AFOSR-FA9550-04-1-0260 and NIDCD (P30) DC04663.]

2pPP26. Effect of number of masking talkers on masking of Chinese speech. Xihong $\mathrm{Wu}$, Jing Chen, Zhigang Yang, and Liang Li (Speech and Hearing Res. Ctr., Peking Univ., Beijing, China, 100871, wxh@cis.pku.edu.cn)

In this study, speech targets were nonsense sentences spoken by a Chinese female, and speech maskers were nonsense sentences spoken by other one, two, three, or four Chinese females. All stimuli were presented by two spatially separated loudspeakers. Using the precedence effect, manipulation of the delay between the two loudspeakers for the masker determined whether the target and masker were perceived as coming from the same or different locations. The results show that the one-talker masker produced the lowest masking effect on Chinese speech. When the number of masking talkers increased progressively to 2,3 , and 4 , even though informational masking progressively decreased, energetic masking progressively increased, leading to an increased total masking effect on targets. A new form of calculation of the speech intelligibility index confirmed an increase of energetic masking as the masking-talker number increased, even when the long-term average signal-to-noise ratio was unchanged. Some differences between Chinese speech masking and English speech masking were revealed by this study. [Work supported by China NSF and Canadian IHR.]

2pPP27. The role of fundamental frequency and target segregation in the presence of same-band and different-band maskers. Nandini Iyer, Douglas Brungart, and Brian Simpson (Air Force Res. Lab., 2610 Seventh St., Bldg 441, Area B, WPAFB, OH 45431, nandini.iyer@wpafb.af.mil)

A number of recent studies on multitalker speech perception have been based on envelope-modulated, sine-wave-excited speech similar to the kind used to simulate the processing used in cochlear implants. Although these modulated sine-band signals are highly intelligible in isolation, they are typically much harder to segregate in multitalker listening tasks than normal speech. In this experiment, we examined the extent to which the difficulty in segregating these signals could be attributed to the absence of the fundamental frequency $F 0$ that is present in natural speech. Psychometric functions were measured with speech and noise maskers for three types of signals: (1) natural speech; (2) sine-excited cochlear implant speech (CIS); and (3) F0-excited CIS, which was generated in the same way as the CIS but with a simulated glottal pulse train as an excitation source. The results show that performance was better with the $F 0$-excited CIS than with the sine-excited CIS in all conditions, including those where the target and masking talkers overlapped in frequency. Performance was also generally better when the $F 0$-excited CIS was generated with $F 0$ values from different-sex rather than same-sex talkers. The results confirm the importance of $F 0$ cues in the segregation of competing speech signals.

2pPP28. Effect of peripheral interference and attentional loss on divided listening to multiple simultaneous talkers. Takayuki Kawashima (Dept. of Cognit. and Behavioral Sci., Univ. of Tokyo, 3-8-1 Komaba, Meguro-ku, Tokyo, 153-8902 Japan, ckawa@mail.ecc.u-tokyo.ac.jp) and Takao Sato (Univ. of Tokyo, Bunkyo-ku, Tokyo, 113-0033 Japan)

Effects of two factors, peripheral interference (masking) and attentional loss, that may impose restriction on divided listening were investigated using Japanese words. In each trial in the divided listening task, simultaneous multiple voices were presented first, then followed by a tar- get voice produced by one speaker. Participants reported whether the target was included in the multiple voices or not. When the number of talkers (manipulated from one to six) was more than three, the estimated hit ratio was lower than unity, and decreased monotonically as the number of talkers increased. To investigate factors inducing the decrement, participants were also tested when the target was presented before the multiple voices. In this condition, decrement of the performance as a function of the number of talkers was gradual, compared to that in the divided listening condition. Decrement of the performance in the pretarget condition was thought to come mainly from peripheral interference, and the difference between the two tested conditions was interpreted as representing the cost in dividing attention. The result suggests that failure in divided listening to three or four talkers largely comes from the attentional factor, although the role of peripheral interference becomes larger when there are more talkers.

2pPP29. Change deafness and how the auditory system organizes sounds. Melissa K. Gregg and Arthur G. Samuel (Dept. of Psych., State Univ. of New York, Stony Brook, Stony Brook, NY 11794-2500, mgregg@ic.sunysb.edu)

Change blindness, or the failure to detect changes to a visual scene that seem blatantly obvious after the fact, has been demonstrated in a variety of different situations. Exploration of auditory failures to detect change has been far less studied, even though demonstrations of change deafness could have important theoretical implications. Four experiments were conducted to explore the processes involved in change deafness by measuring explicit change detection as well as auditory object encoding of natural auditory scenes. Considerable change deafness occurred, even though auditory objects were encoded quite well. Change detection performance was better when stimuli were not presented at distinct spatial locations (experiment 1) than when they were presented with spatial cues (experiment 2). Familiarity with the objects did not affect detection or recognition performance. In order to establish an effective cue for auditory detection and recognition, performance was assessed when auditory stimuli were presented in distinct regions of perceptual space based on fundamental frequency and harmonicity (experiments 3 and 4). Implications for the mechanisms responsible for change deafness and auditory sound organization are discussed.

2pPP30. Sensitivity to increments analyzed separately for each level in a roving paradigm. Anne-Marie Bonnel and Ervin Hafter (Dept of Psych., Univ. of California at Berkeley, 3210 Tolman Hall, Berkeley, CA 94720-1650)

Studies of level discrimination using a dual-task paradigm have shown no cost of shared attention when levels were roved, so that comparisons between targets and standards were in sensory-trace memory, but clear evidence of a cost when levels were fixed, and comparisons seemed based on context-coded memory. Interest in the effects of these two types of memory led here to an auditory single-task in which a trial consisted of a standard tone set to level (I), followed by a target that was (I) alone or (I+\&\#916;I). Durations were 50 or $200 \mathrm{~ms}$; standards ranged from 50 to $70 \mathrm{~dB}$ SPL. In line with the view that the near miss to Weber's Law is negligible over this range, fixed standards provided constant thresholds ( $\log \& \# 916 ; \mathrm{I} / \mathrm{I})$. As typical, overall performance was reduced by roving, but a surprising result was that the magnitude of this loss depended on (I). Thus, roving had the least effect with the highest value of (I); then thresholds rose steeply as (I) was reduced. Independent of this slope, a wider range of roving produced a greater overall loss. This is discussed in terms of an additive noise, possibly connected to the use of trace memory. 
2pPP31. Factors influencing the auditory and cross-modal attentional blink. Adelbert W. Bronkhorst, Marieke van der Hoeven (TNO Human Factors, Kampweg 5, 3769 DE Soesterberg, The Netherlands, adelbert.bronkhorst@tno.nl), Jan Theeuwes, Erik van der Burg, and Thomas Koelewijn (Vrije Univ., 1081 BT Amsterdam, The Netherlands)

When an observer has to report two targets ( $\mathrm{T} 1$ and $\mathrm{T} 2$ ) embedded in a stream of distracters, an attentional blink can occur. This is the degradation in performance for $\mathrm{T} 2$ when $\mathrm{T} 1$ and $\mathrm{T} 2$ are presented sufficiently close in time (within about $500 \mathrm{~ms}$ ). The attentional blink paradigm has been extensively used within the visual domain as a tool to study bottlenecks in the processing of sensory information. Several studies have shown that an attentional blink can also occur during auditory or crossmodal presentation. However, these blinks are smaller and more stimulus dependent than visual blinks. In our research we have found a robust blink when $\mathrm{T} 1$ and $\mathrm{T} 2$ are tones embedded in distracters tones, but no blink when $\mathrm{T} 2$ is replaced by a visually presented letter among different distractor letters. The cross-modal blink is also absent when both $\mathrm{T} 1$ and $\mathrm{T} 2$ are digits. We do find a clear cross-modal blink when a visual T1 with increased difficulty (three letters or a word) is combined with a T2 consisting of a spoken letter. Our results indicate that there are modality-specific as well as amodal bottlenecks and that the latter occur when stimuli are processed on a verbal or semantic level.

2pPP32. Reproducible maskers reveal similar mechanisms predicting multiple-burst-same and multiple-burst-different informational masking conditions. Robert H. Gilkey (Wright State Univ., Dayton, $\mathrm{OH}$ 45435), Christine R. Mason, and Gerald Kidd, Jr. (Boston Univ., Boston, MA 02215)

Gilkey, Mason, and Kidd [J. Acoust. Soc. Am. 109, 2468 (2001)] found that although informational masking has typically been thought to depend more strongly on the variability in the ensemble of masking stimuli presented across trials, trial-by-trial performance was strongly related to the specific masker waveform presented on each trial. Moreover, hit and false-alarm rates to individual masker stimuli under multiple-burstsame (MBS) conditions were well predicted by the patterns of energy in those stimuli. Hit and false-alarm rates under the multiple-burst-different (MBD) condition were more difficult to predict. This paper reports on further efforts to model those data. The results indicate that performance under both MBS and MBD conditions can be predicted using a linear combination of envelope statistics computed from seven narrow frequency bands surrounding the $1000-\mathrm{Hz}$ signal frequency. MBS responses are related to the average height of the envelope in these bands and MBD responses are related to the standard deviations of the envelopes. [Work supported by NIH/NIDCD.]

2pPP33. The application of the equal energy hypothesis (EEH) to interrupted, intermittent, and time-varying non-Gaussian noise exposures. Roger P. Hamernik, Wei Qiu, and Robert I. Davis (Auditory Res. Lab., State Univ. of New York at Plattsburgh, 101 Broad St., Plattsburgh, NY 12901)

Industrial data and animal research show that non-Gaussian (nonG) noise exposures are more hazardous to hearing than energy equivalent Gaussian $(\mathrm{G})$ exposures. A statistical metric, kurtosis $[b(t)]$, was shown to order the severity of noise-induced trauma following a nonG exposure relative to an energy and spectrally equivalent $\mathrm{G}$ exposure. Four groups of chinchillas were exposed to one of four different nonG interrupted, intermittent, and time-varying (IITV) noise paradigms over 19 days at an Leq $=103 \mathrm{~dB}(\mathrm{~A}) \mathrm{SPL}$, with $b(t)=25$ or 50 . Each daily exposure consisted of two 4.25-h periods with an hour break. Each 4.25-h exposure was interrupted for $15 \mathrm{~min}$ and each 5-day sequence was separated by a 2-day break. Each daily IITV exposure followed one of two different SPL temporal patterns that varied between 90 and $108 \mathrm{~dB}(\mathrm{~A})$. All IITV exposures produced a toughening effect that did not alter the degree of noise-induced trauma. NonG noise produced as much trauma as a $\mathrm{G}$ exposure at $110 \mathrm{~dB}$.
Despite very different temporal paterrns for the $b(t)=50$ exposures, trauma was the same. Thus within a common class [i.e., the same Leq and $b(t)]$ of nonG, IITV exposure, the EEH may apply. [Work supported by NIOSH.]

2pPP34. The application of statistical learning models to the prediction of noise-induced hearing loss. Wei Qiu, Jun Ye, and Roger P. Hamernik (Auditory Res. Lab., State Univ. of New York at Plattsburgh, 101 Broad St., Plattsburgh, NY 12901)

Three powerful nonlinear statistical algorithms [a support vector machine (SVM), radial basis function network (RBFN), and regression tree] were used to build prediction models for noise-induced hearing loss (NIHL). The models were developed from an animal (chinchilla) database consisting of 322 animals exposed to 30 Gaussian and non-Gaussian noise conditions. The inputs for the models were either energy or energy plus kurtosis. The models predict inner hair cell (IHC) loss, outer hair cell (OHC) loss, and postexposure threshold shift (PTS) at 0.5, 1, 2, 4, and 8 $\mathrm{kHz}$. The models incorporating both energy and kurtosis improved the prediction performance significantly. The average performance improvement for the prediction of IHC loss was as much as 55\%, for OHC loss it was $66 \%$ and for PTS, $61 \%$. The prediction accuracy of SVM and RBFN with energy plus kurtosis for all three outputs (predictions) was more than $90 \%$ while for the regression tree model it was more than $85 \%$. Energy is not a sufficient metric to predict hearing trauma from complex (nonGaussian) noise exposure. A kurtosis metric may be necessary for the prediction of NIHL. [Research supported by NIOSH.]

2pPP35. The development of models for the prediction of noiseinduced hearing loss. Wei Qiu, Jun Ye, and Roger P. Hamernik (Auditory Res. Lab., State Univ. of New York at Plattsburgh, 101 Broad St., Plattsburgh, NY 12901)

Three statistical learning models were developed to predict noiseinduced hearing loss (NIHL) from an archive of animal noise exposure data, which contains 936 chinchillas exposed to various noise environments. The following models were constructed: (i) A support vector machine model with a nonlinear radial basis function kernel. (ii) A multilayer perceptron network model and (iii) a radial basis function network model. In addition to frequency-specific energy metrics, noise exposure parameters and biological metrics such as kurtosis, noise type, and pre/ postexposure hearing thresholds were used as inputs to the model. There were several indices of auditory trauma at specific audiometric test frequencies that were to be predicted by the models: e.g., noise-induced permanent threshold shift, percent outer hair cell loss, and percent inner hair cell loss. The average prediction accuracy for the three models was better than $80 \%$. These results demonstrate the feasibility of developing such models for the prediction of NIHL in humans. [Research supported by NIOSH.]

2pPP36. Noise-induced hearing loss from non-Gaussian equal energy exposures. Robert I. Davis, Wei Qiu, and Roger P. Hamernik (Auditory Res. Lab., State Univ. of New York at Plattsburgh, 101 Broad St., Plattsburgh, NY 12901)

Data from several different exposures [Hamernik et al., J. Acoust. Soc. Am. 114, 386-395 (2003)] showed that, for equivalent energy [Leq $=100 \mathrm{~dB}(\mathrm{~A})]$ and spectra, exposure to a continuous, non-Gaussian (nonG) noise produces greater hearing and sensory cell loss in the chinchilla than a Gaussian (G) noise. The statistical metric, kurtosis, could order the extent of the trauma. We extend these results to Leq $=90$ and $110 \mathrm{~dB}(\mathrm{~A})$, non-Gaussian noises generated using broadband noise bursts, and bandlimited impacts within a continuous $\mathrm{G}$ background noise. Data from nine new experimental groups with 11 or 12 chinchillas/group will be presented. Evoked response audiometry established hearing thresholds and surface preparation histology quantified sensory cell loss. There were clear 
intensity-related effects. At the lowest levels there were no differences in the trauma produced by $\mathrm{G}$ and nonG exposures. At Leq $=90 \mathrm{~dB}(\mathrm{~A})$, nonG exposures produced increased trauma relative to equivalent $\mathrm{G}$ exposures. By removing energy from the impulsive transients by limiting their bandwidth, trauma could be reduced. The use of noise bursts to produce the nonG noise also reduced the amount of trauma. A metric based on kurtosis and energy may be useful in modifying existing exposure criteria. [Work supported by NIOSH.]

2pPP37. The application of the equal energy hypothesis (EEH) to interrupted, intermittent and time-varying Gaussian noise exposures. Wei Qiu, Roger P. Hamernik, and Robert I. Davis (Auditory Res. Lab., State Univ. of New York at Plattsburgh, 101 Broad St., Plattsburgh, NY 12901)

Interrupted, intermittent, time-varying (IITV) noise exposures may reduce the susceptibility of the cochlea to hearing loss through toughening or conditioning effects. Six groups of chinchillas were exposed to a broadband Gaussian IITV noise over the course of 19 days at an Leq=101 or $106 \mathrm{~dB}(\mathrm{~A})$ SPL. All exposures at a given Leq had the same total energy. Each daily exposure consisted of two 4.25-h periods with an hour break in between. Each 4.25-h exposure was interrupted for $15 \mathrm{~min}$ and each 5-day sequence was separated by a 2-day break. Daily IITV exposures at $101 \mathrm{~dB}$ varied between 90 and $108 \mathrm{~dB}(\mathrm{~A})$, while those at $106 \mathrm{~dB}(\mathrm{~A})$ varied between 80 and $115 \mathrm{~dB}(\mathrm{~A})$. For the IITV exposures three different variations in the SPL temporal profile were used. Five-day continuous, equal energy exposures at 100 or $106 \mathrm{~dB}(\mathrm{~A})$ SPL served as control conditions. The IITV exposures produced up to $40 \mathrm{~dB}$ toughening that did not have any effect on thresholds or sensory cell losses. There were some differences in the permanent threshold shift and cell loss across equal energy exposures but the differences were small. The EEH may be valid for Gaussian IITV exposures. [Work supported by NIOSH.]

2pPP38. Temporal characteristics of extraction of size information in speech sounds. Chihiro Takeshima (Grad. School of Music, Kyoto City Univ. of Arts, Kyoto 610-1197, Japan, ctakeshima@yahoo.co.jp), Minoru Tsuzaki (Kyoto City Univ. of Arts, Kyoto 610-1197, Japan), and Toshio Irino (Wakayama Univ., Wakayama 640-8510 Japan)

We can identify vowels pronounced by speakers with any size vocal tract. Together, we can discriminate the different sizes of vocal tracts. To simulate these abilities, a computational model has been proposed in which size information is extracted and separated from the shape information. It is important to investigate temporal characteristics of the size extraction process. Experiments were performed for listeners to detect the size modulation in vowel sequences. All the sequences had six segments. Each segment contained one of three Japanese vowels: "a," "i," and "u." Size modulation was applied by dilating or compressing the frequency axis of continuous, STRAIGHT spectra. Modulation was achieved by changing the dilation/compression factor in sinusoidal functions. The original $F 0$ pattern of the base sequence, except for warping of the time axis, was used for all stimuli. The minimum modulation depth at which listeners were able to detect the existence of modulation was measured as a function of the modulation frequency. The results will be compared with low-pass characteristics in a temporal modulation transfer function obtained with the amplitude-modulated noise. They will be discussed in relation to a computational model based on the Mellin transformation.

2pPP39. She hears seashells: Detection of small resonant cavities via ambient sound. Ethan J. Chamberlain, Lawrence D. Rosenblum, and Ryan L. Robart (Dept. of Psych., Univ. of California, Riverside, Riverside, CA 92521)

There is evidence that blind listeners can detect the presence of obstacles based on how the obstacles structure the ambient sound in a quiet room [Ashmead et al., J. Vis. Impair. Blindn. 9, 615 (1998)]. Potentially, this ability is based on auditory sensitivity to the buildup of ambient wave interference patterns in front of the obstacle. This effect is not unlike how the internal structure of a seashell amplifies a room's ambient sound to emulate the sound of the ocean. Experiments were conducted to determine whether unpracticed, sighted listeners were sensitive to this information. Blindfolded listeners were placed in a quiet room and were asked to determine the location of a small resonant cavity placed next to either their left or right ear. Results revealed that listeners were very accurate at this task. Follow-up experiments examined the limits of this sensitivity as well as its acoustical support. These findings suggest that unpracticed listeners might be sensitive to subtle changes in the ambient acoustic structure of a quiet environment.

2pPP40. Effects of listening environment on speech recognition in noise. Linda Thibodeau and Tina Keene (Univ. of Texas at Dallas, 1966 Inwood Rd., Dallas, TX 75235)

The ability to recognize speech in noisy situations varies among individuals despite normal hearing. Although some of the variation may be related to physiological differences, some may be attributed to environmental experiences. The purpose of this investigation was to examine how everyday listening experiences were related to speech recognition in noise abilities. Using the Hearing in Noise Test, reception thresholds for speech were compared for listener groups who differed in their preferred listening levels for music and signal-to-noise ratios for daily communication. Results suggested that speech recognition in noise was related to listening levels. These results have implications for the use of assistive devices to improve the signal-to-noise ratio in adverse communication situations.

2pPP41. Comparing vowel formant thresholds from two tasks: Classification versus 2-alternative forced choice (2AFC) adaptive tracking. Eric Oglesbee (Dept. of Linguist., Indiana Univ., Bloomington, IN 47405, eoglesbe@indiana.edu) and Diane Kewley-Port (Indiana Univ., Bloomington, IN 47405)

Accurate classification of vowels in American English is difficult because of the number of acoustically similar vowels. Previous experiments in our lab [Kewley-Port et al., J. Acoust. Soc. Am. 118, 1929-1930 (2005)] described a new method to obtain formant thresholds using a classification task rather than the more typical 2AFC task. The goal of the present experiment was to directly compare formant thresholds estimated from a classification task with a modified $2 \mathrm{AFC}$ paradigm using adaptive tracking. Stimuli, generated by STRAIGHT, consisted of separate continua for formants F1 and F2 based on natural productions of "bid" shifted to "bed," and "cut" shifted to "cot." Separate 7- and 14-step continua were created for the classification and 2AFC tasks, respectively. Eight subjects participated first in the classification task, followed by the 2AFC task. Classification threshold results here replicated those observed in our previous experiments. Within-subject comparisons of the classification and 2AFC data refined our method for calculating a classification threshold from logistic psychometric functions. Individual thresholds calculated using this refined method were very similar to those from the $2 \mathrm{AFC}$ task. Results demonstrate the viability of using a more natural, single-interval classification task to estimate formant discrimination thresholds. [Work supported by Grant NIHDCD-02229.]

2pPP42. Cochlear and cognitive models for speech enhancement. Melissa Dominguez (DBK Acoust., 110 Village Station Ln., Grayslake, IL, 60030), Jeff Bondy, and Andrew Dittberner (GN Resound, Glenview, IL, 60026)

There has been a long running interest in using cochlear models for audio processing. Psychophysical masking stemming from normal cochlear operation is used as the basis for most audio compression circuits, while the cochleas mel-frequency response is mimicked by most automatic speech recognition circuits. An area that has been less well explored 
in audio processing is the auditory brain. Recent research shows that cognitive abilities are a good predictor of peformance on many hearing tasks, especially those which are more complex and realistic. By extending a nonlinear, adaptive cochlear model with cognitive modules a novel tool for sound assessment is made that allows an estimate of cognitive load. Quantitative offline benefit has never been modeled before. We test the new predictor on noise reduction circuits in hearing aids. This family of algorithms is well known to not increase intelligibility but has been shown to reduce perceived noisiness. This reduction is proposed as equivalent to reducing cognitive load. The elderly, with their heavier reliance on high level processing, could greatly benefit from the reduction in cognitive load. A new algorithm for reducing cognitive load is compared to spectral subtraction algorithms.

2pPP43. Cross-modal plasticity and the sensitive period for cochlear implantation. Kristi A. Buckley, Emily A. Tobey (The Univ. of Texas at Dallas, 1966 Inwood Rd. Dallas, TX 75235), and Anu Sharma (The Univ. of Colorado at Boulder)

Previous research suggests prelingually deaf individuals who receive a cochlear implant early in childhood develop better oral communication skills than those who receive their implant later. However, the mechanisms influencing this sensitive period are not well understood. This investigation explores one possible mechanism limiting the sensitive period for cochlear implantation, cross-modal plasticity. Cross-modal plasticity occurs when one sensory system recruits cortical processing resources of another absent or damaged sensory system. This reallocation of cortical processing resources may influence the ability of the auditory system to respond to restored auditory sensation through the electrical stimulation of a cochlear implant. In order to investigate the possible relationship between cross-modal plasticity and the sensitive period for cochlear implantation, we examined the visual evoked potential (VEP) to peripheral motion in prelingually deaf children and adults, and normal-hearing children and adults. The VEP response source is compared across ages and groups. Preliminary data from adults indicate the source of the VEP response in deaf individuals originates from the temporal lobe, compared to the parietal lobe in normal hearing individuals, suggesting evidence of cortical reorganization in deafness. [Work funded by NIH F31DC007007 (K. Buckley, P.I.), R01DC04558 (E. Tobey, P.I.), and R01DC006257 (A. Sharma P.I.).]

2pPP44. Predictions of Mandarin Chinese tone identification in cochlear implants using particle filters. Kenneth D. Morton, Jr., and Leslie M. Collins (Dept. of Elec. and Comput. Eng., Duke Univ., 130 Hudson Hall, Box 90291, Duke Univ., NC 27708)

The spectral information provided in current cochlear implants is inadequate for reliable speech perception in tonal languages. Speech comprehension in a tonal language, such as Mandarin Chinese, requires recognition of lexical tones. New strategies for sound processing may allow the transmission of more spectral information and may therefore improve Mandarin Chinese tone recognition. Four cochlear implant speech processing strategies were examined in this study. Each provides a different amount of spectral information. Automated pattern recognition of Mandarin Chinese tones processed using cochlear implant acoustic models can be used as a means of testing the abilities of each of the algorithms to transmit the necessary spectral information for accurate tone perception and may be used to focus future Mandarin Chinese tone recognition studies in cochlear implant subjects on the most promising speech processing strategies. Classification based on spectral features derived from particle filtering is compared to classification derived from other features in an effort to determine the necessary spectral information for accurate tone classification in both quiet and noisy conditions. Automated classification results will be compared to the results of a listening experiment using normalhearing subjects and acoustic models.
2pPP45. Simulating the effect binaural (electric and acoustic) hearing on Mandarin speech recognition under background noise for patients with cochlear implant (CI). Chao-Min Wu and Wei Jen Cheng (Dept. of Elec. Engr., Natl. Central Univ., 300, Chung-Da Rd., Chung-Li, Taiwan 320)

This study evaluated the effects of binaurally combined electric and acoustic hearing on Mandarin speech recognition under simulated background noise for normal-hearing $(\mathrm{NH})$ listeners and $\mathrm{CI}$ recipients of the Nucleus-24 device with contralateral hearing aid (HA). Chinese word and tone recognition were measured in seven $\mathrm{NH}$ subjects using simulated four-channel continuous interleaved sampler (CIS) type speech processing strategy under three different conditions, namely, (1) only simulated (CI) signals with modulated Gaussian white noise for monaural stimulation, (2) simulated (CI) signals with modulated Gaussian white noise and simulated HA signals (low-pass speech signals with cutoff frequency of $500 \mathrm{~Hz}$ ) for monaural stimulation, and (3) simulated (CI) signals with modulated Gaussian white noise to one ear and simulated hearing aid (HA) signals to the other ear for dichotic stimulation. For comparison purposes, Chinese word and tone recognition were also measured in three CI users using advanced combination encoding (ACE) strategy. Preliminary results showed that the Chinese word and tone recognition rates of the NH listeners and CI recipients are statistically significantly improved (word and tone recognition rates were improved from $3.85 \%$ to $60.86 \%$ and $40.14 \%$ to $97.43 \%$ for $\mathrm{NH}$ listeners and $20 \%$ to $37.5 \%$ and $50 \%$ to $90 \%$ for CI recipients).

2pPP46. Pitch discrimination in children using cochlear implants. Amy McKinnon and Michael Kiefte (School of Human Commun. Disord., Dalhousie Univ., 5599 Fenwick St., Halifax, NS, B3H 1R2 Canada)

Research in pitch perception by cochlear implant users has focused primarily on postlingually deafened adults. Little is currently known regarding the pitch perception abilities of those who are implanted at a very early age, yet many children receive cochlear implants when they are less than 2 years old. Given the effects of brain plasticity, it is conceivable that pitch perception by early cochlear implant users may be much better than that of their older counterparts. This study examines pitch discrimination thresholds in young cochlear-implant users between the ages of 4 and 16 . A computer game was developed to determine difference limens for fundamental frequency in vowellike stimuli at three referent frequencies: 100, 200, and $400 \mathrm{~Hz}$. Two different tasks were employed: pitchdiscrimination, in which subjects were asked whether two stimuli are same or different, and pitch ranking, in which participants determine which of two stimuli is higher in pitch. Normal-hearing matches were tested to control for maturational effects, and adult cochlear implant users were also tested to compare findings with other studies. [Work supported by the Nova Scotia Health Research Foundation.]

2pPP47. Improving the voice pitch discrimination threshold through cochlear implants. Shizuo Hiki (Waseda Univ., Tokorozawa, Japan, hiki@waseda.jp) and Masae Shiroma (Natl. Univ. of Health and Welfare, Ohtawara, Japan)

Aiming at the maximum use of linguistic information conveyed by the periodicity pitch of speech sound through cochlear implants, the possibility of improving the voice pitch discrimination threshold was examined for cochlear implantees: three acquired deaf adults and four congenitally deaf children. Female and male adult speech samples of vowel /a/ were analyzed and resynthesized using the STRAIGHT computer program to shift the original voice pitch ( $\mathrm{c} 1$ and $\mathrm{c}$ ) one octave upward and downward with a half-step interval on the musical scale. By presenting those synthetic speech samples in pairs, the discrimination threshold of the voice pitch interval was observed. Association of the speech samples to the picture of a piano keyboard was helpful for conceptualizing the voice pitch change, especially for children. Preliminary observation results suggested that the discrimination threshold can be improved by less than one 
whole step by adults and two whole steps by children. Those figures were promising because, even for Japanese word accent and Chinese tones, which were conveyed by the precise changes in voice pitch, it was ascertained by acoustical analyses of both their production and perception that discrimination of a half-step difference was small enough to recognize their pitch patterns.

2pPP48. Implementing a psychophysical interface for the sound processor for electrical and acoustic research version 3 (SPEAR3). Joshua S. Stohl, Chandra S. Throckmorton, and Leslie Collins (Duke Univ., 130 Hudson Hall, Box 90291, Durham, NC 27708; lcollins@ee.duke.edu)

Current speech processing strategies use amplitude-modulated fixed rate pulse trains to communicate information. Using acoustic models, researchers have recently demonstrated that variable stimulation rate strate- gies may provide an improvement of speech perception in noise and music perception [Nie et al., 2005; Stohl and Collins, 2006; Throckmorton et al. (2006) (in press)]. It has been hypothesized that successful implementation of these strategies will require psychophysics-based tuning. An experimental interface is needed to implement such psychophysical experiments, and most interfaces provided by implant manufacturers do not provide the stimulus design flexibility necessary. HearWorks provides the SPEAR3 developmental sound processor, which is capable of dynamically updating the stimulation rate and offers bimodal and bilateral configurations. Assembly and Visual Basic code is included with the SPEAR3, but must be modified and expanded to implement dynamic stimulus presentation and graphical user interfaces for specific psychophysical tasks. Using data collected from Nucleus CI 24 implant users, we will demonstrate that the SPEAR3 may be used to implement general psychophysical experiments. The ability of the SPEAR3 speech processor to implement variable stimulation rates is critical to future psychophysical testing of variable stimulation rate processing strategies.

WEDNESDAY AFTERNOON, 29 NOVEMBER 2006

REGENCY BALLROOM (ROYAL HAWAIIAN), 2:00 TO 4:45 P.M.

\author{
Session 2pSA \\ Structural Acoustics and Vibration: Scattering, Diffraction, Radiation, and Damping \\ Sabih I. Hayek, Cochair \\ Pennsylvania State Univ., Dept. of Engineering Science and Mechanics, 212 Earth Engineering Science Bldg., \\ State College, PA 16802-6812 \\ Katsuji Akamatsu, Cochair \\ Machinery Acoustics, 1-1-2-314 Obanoyama, Shinohara, Nada-ku, Kobe 657-0015, Japan
}

\title{
Contributed Papers
}

2:00

2pSA1. Phased array steering of structure-borne sound radiated from a flexible beam by active modal control. Alfred Tan (Dept. of Aerosp. Eng., Tokyo Metropolitan Univ., 6-6 Asahigaoka, Hino-shi, Tokyo, 191-0065, Japan), Dunant Halim (The Univ. of Adelaide, SA 5005, Australia), and Nobuo Tanaka (Tokyo Metropolitan Univ., Hino-shi, Tokyo, 191-0065, Japan)

This paper analytically demonstrates the implementation of a control strategy to actively steer the structure-borne sound radiated from a simply supported beam structure, which is excited by an arbitrary monofrequency force. The surface of the beam is discretized into many finite elemental sound radiators that correspond to their respective nodal points. The dynamics of the beam structure are identified in order to modify the modal vibration characteristic for beamsteering purpose. The acoustic power is formulated in terms of its velocity according to the Rayleigh expression. The key to the control strategy is to combine the a priori knowledge of the structural modal characteristic with the sensors measurements. As a result, this is used to control the normal surface vibration velocity profile of the beam structure into a set of congruent elemental sound radiators, each differing only by successive delayed phase in order to steer the radiated sound beam pattern into a desired far field direction. The arrangement of the sensors/actuators on the beam structure is designed to instill such a unique set of elemental sound radiators. Numerical studies are performed to evaluate the effectiveness of the proposed sound steering concept into the far-field hemisphere. [Work supported by JSPS.]

\section{2:15}

2pSA2. Effects of material orientation on the radiation efficiency of baffled plates. J. Gregory McDaniel (Dept. of Aerosp. and Mech Eng., Boston Univ., 110 Cummington St., Boston, MA 02215, jgm@bu.edu)

The orientation of an anisotropic material may have profound effects on acoustic radiation from the structure. The present work explores these effects for the case of a thin anisotropic plate in an infinite rigid baffle. An understanding of the acoustic radiation, in terms of the radiation efficiency, is begun by considering two effects. The first is that of wave steering, in which the direction of structural wave propagation is changed by the material orientation. The second is that of wave mixing, in which the the relative mixture of propagating and evanescent waves is changed by the material orientation. The effect on radiation follows from the fact that radiation from propagating and evanescent waves is known to be dramatically different. The relative importance of frequency and excitation is also explored in the context of these two effects. These results are expected to lead to improved designs of composite structures at no additional cost-simply by changing the orientation of the material. [Work supported by ONR.] 
2:30

2pSA3. A method to measure the equivalent acoustic impedance of a scatterer. Seung-ha Lee (Ctr. for Noise and Vib. Control, Dept., of Mech. Eng., KAIST, Sci. Town, Daejeon, 305-701, Korea, seung-habird@kaist.ac.kr)

The procedures of simplifying scatterers' geometry and finding its equivalent acoustic impedance are proposed by using scattering holography and multiple multipole expansions. The scattered sound field depends on the incident field, the geometry, and the physical property of the scatterer, which can be measured by using tools such as a set of microphones and a 3-D scanner. Once the sound field over a surface is measured, the whole sound field of 3-D space can be reconstructed by acoustic holography. However, predicting near-field sound by holography is inefficient in practice, because huge numbers of measurement points are required to describe the complex sound field in the vicinity of a scatterer. With this regard, multiple multipole expansions are employed to reduce the number and the size of elements of a scatterer necessary to express the measured scattered field. Therefore it is equivalent to simplifying the geometry of a scatterer, and enables us to predict the sound pressure and velocity, as well as acoustic impedance, on the scatterers' surface. Using the proposed method, it is demonstrated that the equivalent acoustic impedance can be obtained. [Work supported by the BK21 project initiated by the Ministry of Education and Human Resources Development of Korea.]

\section{2:45}

2pSA4. Acoustic backscattering from fluid-filled submerged prolate spheroidal shells. Jeffrey E. Boisvert (NAVSEA Newport, Newport, RI 02841) and Sabih I. Hayek (Penn State Univ., University Park, PA 16802)

The equations of motion for nonaxisymmetric vibration of prolate spheroidal shells of constant thickness were derived using Hamilton's principle [S. I. Hayek and J. E. Boisvert, J. Acoust. Soc. Am. 114, 27992811 (2003)]. The shell theory used in this derivation includes shear deformations and rotatory inertias. The shell displacements and rotations were expanded in infinite series of comparison functions. These include associated Legendre functions in terms of the prolate spheroidal angular coordinate and circular functions in the azimuthal angle coordinate. The fluid-filled shell is insonified by a plane wave with an arbitrary angle of incidence. The external and internal fluid loading impedances were computed using an eigenfunction expansion of prolate spheroidal wavefunctions. Far-field backscattered acoustic pressure spectra are presented as a function of the angle of incidence for several shell thickness-to-half-length ratios ranging from 0.005 to 0.1 , and for various shape parameters, $a$, ranging from an elongated spheroidal shell $(a=1.01)$ to a spherical shell $(a \sim 100)$. A comparison of the backscattering from fluid-filled and empty shells is presented at selected plane wave incident angles. [Work supported by the ONR/ASEE Summer Faculty Research Program and the NAVSEA Newport ILIR Program.]

\section{3:00}

2pSA5. Modeling a magnetorheological elastomer's transient stiffness parameters. Anne-Marie Lerner and Ken Cunefare (Georgia Tech., ken.cunefare@me.gatech.edu)

Semiactive vibration absorbers are tunable vibration absorbers whose natural frequencies can change controllably to minimize vibration better than their passive counterparts, which can be used to reduce occupant vibration in cars. The variable natural frequency is achieved by using a dynamically tunable spring. In order to use a material as a dynamically tunable spring, its transient parameters must be understood and controlled, so as not to lead to instability. This work characterizes the behavior of magnetorheological elastomers (MREs), which are a type of ferromagnetic-elastomer composites whose tensile/compressional stiffness increases as an applied magnetic field is increased. If the transition from one stiffness state to another is too slow, the MRE can have a detrimental effect on vibration control. Transient behavior of MREs with different concentrations of ferromagnetic material and different geometric shapes was recorded empirically, and the MRE stiffness change in response to a change in magnetic field was modeled as a function of ferromagnetic content, MRE geometric shape, and the change in magnetic field strength. MREs in this configuration exhibited natural frequencies in the $30-65-\mathrm{Hz}$ range, where MREs were able to change their natural frequency by up to $17 \%$.

\section{3:15-3:30 Break}

\section{$3: 30$}

2pSA6. Vibration absorption by an undamped beam element. Ilker Murat Koc, Adnan Akay (Dept. of Mech. Eng., Carnegie Mellon Univ., Pittsburgh, PA 15213), and Antonio Carcaterra (Univ. of Rome, La Sapienza, 00184, Rome, Italy)

Through two complementary approaches, using modal response and wave propagation, this presentation will show the conditions under which a decaying impulse response, or a nearly irreversible energy trapping, takes place in a linear conservative continuous system. The results show that the basic foundation of near-irreversibility or pseudo-damping rests upon the presence of singularity points in the modal density of the systems. To illustrate the concept of pseudo-damping in detail, a simple undamped beam is modified to introduce a singularity point in its modal density distribution. Simulations show that by a suitable application of a compressive axial force to an undamped beam on an elastic foundation, the impulse response of a beam shows the characteristics of a nearly irreversible energy trap and a decaying impulse response. Attaching such a modified beam to a single degree of freedom oscillator shows energy trapping by the beam producing pseudo damping. [Research carried out while AA served at NSF.]

\section{$3: 45$}

2pSA7. Pseudo-damping in undamped plates and shells. Antonio Carcaterra (Dept. of Mech. and Aeronautics, Univ. of Rome, La Sapienza Via Eudossiana, 18, 00184, Rome, Italy) and Adnan Akay (Carnegie Mellon Univ., Pittsburgh, PA 15213)

Pseudo-damping is a counter-intuitive phenomenon observed in a special class of linear structures that exhibit an impulse response characterized by a decaying amplitude, even in the absence of any dissipation mechanism. The conserved energy remains within but designated parts of the system. Pseudo-damping develops when the natural frequency distribution of the system includes condensation points. The recently formulated theoretical foundation of this phenomenon, based on mathematical properties of special trigonometric series, makes it possible to describe a class of mechanical systems capable of displaying pseudo-damping characteristics. They include systems with discrete oscillators and onedimensional continuous beamlike structures already reported by the authors in recent studies. This paper examines development of pseudodamping phenomenon in two-dimensional structures, using plates and shells as examples, and shows how a preloaded plate on an elastic foundation can lead to pseudo-damping. Moreover, in the case of curved shell elements examined here, pseudo-damping can result due to the curvature of the structure, which naturally introduces condensation points in the modal density. [Research carried out while AA served at NSF.]

\section{4:00}

2pSA8. Fundamentals of apparent damping phenomena in linear conservative systems. Antonio Carcaterra (Dept. of Mech. and Aeronautics, Univ. of Rome, La Sapienza Via Eudossiana, 18, 00184, Rome, Italy) and Adnan Akay (Carnegie Mellon Univ., Pittsburgh, PA 15213)

This presentation addresses a class of irreversible phenomena that can develop in linear conservative systems and provides a theoretical foundation to explain the underlying principles. Recent studies have shown that energy can be introduced to a linear system with near irreversibility, or energy within a system can migrate to a subsystem nearly irreversibly, even in the absence of dissipation. Inspired by the properties of probability distribution functions, the general formulation developed here is based on particular properties of harmonic series, which form the common basis of 
linear dynamic system models. The results demonstrate the existence of a special class of linear nondissipative dynamic systems that exhibit nearly irreversible energy exchange and possess a decaying impulse response. The formulation and its results also support the recent studies that observed near irreversibility and apparent damping in several dynamic systems and provide a common theoretical foundation for such behavior. [Research carried out while AA served at NSF.]

\section{4:15}

2pSA9. Using an enhanced constrained layer damping model to assess relative damping contributions. Zhengchao Xie and W. Steve Shepard , Jr. (Dept. of Mech. Eng., The Univ. of Alabama, Box 870276, Tuscaloosa, AL 35487, sshepard@eng.ua.edu)

Constrained layer damping (CLD) is an effective passive vibration control approach in which damping comes from the core layer's deformation. In the most general sense, this deformation will include shear, longitudinal extension, and transverse compression deformations. In this work, new analytical models and their single-layer finite-element (FE) implementations are used to examine the dependence of these types of damping on the frequency and the core layer's thickness for different boundary conditions. This information can be important in cases where a simplified model is needed with an acceptable error. The sandwich beam's response is examined by using various tools in order to determine the important aspects of the model, with particular emphasis on the relative importance of each of the three types of damping. Then, frequency responses found using different single-layer finite elements are compared for different boundary conditions. By using both the analytical models and FE method results, guidance is provided on how to choose an appropriately simplified model or finite element for more practical analyses.

\section{4:30}

2pSA10. Effect of burst-modulated sinusoidal dither waveforms on the effectiveness of dither forces to eliminate friction-induced oscillations. Jeff W. Badertscher, Kenneth A. Cunefare, Al A. Ferri, and Michael M. Michaux (771 Ferst Dr., Rm. 205, Altanta, GA 30332)

This paper examines the effectiveness of high-frequency dithercancellation techniques using burst-modulated signals. The classic singledegree-of-freedom (SDOF), mass on moving belt, model is analyzed using the method of averaging and numerical integration of system dynamics. Two different friction laws are used to model the contact point, both exhibiting a negative friction coefficient-velocity relationship. Recent results [M. Michaux and A. Ferri, Proceedings of IDETC05 Paper no. DETC2005-84491 (2005)] found that sinusoidal dither forces could stabilize or destabilize such a system, depending on the system and frictional characteristics as well as the dither signal amplitude and frequency. This paper extends this previous analysis to burst-modulated sinusoidal dither signals. Earlier experimental research has shown burst-modulated dither signals to be an effective method of suppressing automotive brake squeal. It is found that, for a given amplitude and frequency, burst signals are also capable of stabilizing the system. Individual effects of each burst parameter on system stability are established.

WEDNESDAY AFTERNOON, 29 NOVEMBER 2006

MOLOKAI ROOM, 1:00 TO 2:00 P.M.

\title{
Session 2pSCa
}

\section{Speech Communication: First Language Acquisition (Poster Session)}

\author{
Megha Sundara, Cochair \\ Univ. of Washington, Inst. for Learning and Brain Science, Box 357988, Seattle, WA 98195-7988 \\ Kazuhiko Kakehi, Cochair \\ Chukyo Univ., Tokodachi 101, Toyota 470-0393, Japan
}

\section{Contributed Papers}

\begin{abstract}
All posters will be on display and all contributors will be at their posters from 1:00 p.m. to 2:00 p.m. To allow for extended viewing time, posters will remain on display until 5:30 p.m.
\end{abstract}

2pSCa1. When is a phonological rule acquired? A look at children's production on syllable-final nasals in Taiwan Mandarin. Renee Hung (Dept. of English, Natl. Taiwan Normal Univ., 162 Hoping E. Rd., Sec. 1, Taipei, Taiwan 106, reneejh@ @otmail.com) and Janice Fon (Natl. Taiwan Univ. Taipei, Taiwan 106)

Syllable-final nasals, $/ \mathrm{n} /$ and $/ \mathrm{N} /$, tend to be merged when they are after /i/ and $ə$ in Taiwan Mandarin, but in different directions. For $/ \mathrm{i} /, / \mathrm{n} / \mathrm{is}$ merged with /N/, while for $\partial$, /N/ is merged with /n/ (Chen, 1991; Tse, 1992; Hsu, 2005). This study thus aims to examine whether children in Taiwan merge syllable-final nasals in Mandarin the way adults do. Fifty Grade-2 and Grade- 6 students would read monosyllabic words, containing 3 [vowels $(/ \mathrm{i} /, 2, / \mathrm{a} /)] \times 4$ (tones) $=12$ minimal pairs differing only in nasal endings. They would repeat the words twice, once in Zhuyinfuhao, a local phonetic system, and once in traditional Chinese characters. Preliminary results indicated that Grade-2 children treated the /eN/ words very similarly to adults, although the occurrences of nasal merge were less frequent in the Zhuyinfuhao set than in the character set. For vowel /i/, however, merging of both directions could be found in both Mandarin characters and Zhuyinfuhao.

2pSCa2. Phonetic development of word-initial Korean stops in young Korean children. Minjung Kim and Carol Stoel-Gammon (Dept. of Speech \& Hearing Sci., Univ. of Washington, 1417 NE 42nd St., Seattle, WA 98105-6246, minjungk@u.washington.edu)

A number of studies have investigated developmental patterns of stops in various languages through the instrumental analysis; however, very little is known about phonetic characteristics of Korean stops in speech of young Korean children. In Korean, stops are produced at three places of articulation (labial, alveolar, velar); at each place, there is a three-way contrast, yielding nine stops in word-initial position. All stops are voiceless and are differentiated by several acoustic parameters including VOT, f0 of the following vowel, and the amplitude difference of the first and 
second harmonics $(\mathrm{H} 1-\mathrm{H} 2)$ at the voice onset of the following vowel. The present study examines developmental aspects of word-initial Korean stops by measuring values of VOT, f0, and $\mathrm{H} 1-\mathrm{H} 2$ at the onset of the following vowel in words produced by Korean children aged 30, 36, 42, and 48 months. Findings will focus on the phonetic features used by Korean children to contrast the three-way stops and on comparisons between the phonetic patterns of stops produced by Korean children and Korean adults.

2pSCa3. Patterns of anticipatory lingual coarticulation in typically developing children. Shawn Nissen, Kurtt Boucher, and Mathew Parson (Dept. of Commun. Disord., Brigham Young Univ., Provo, UT 84602)

Many studies have examined the patterns of speech coarticulation in adults and younger speakers; however previous findings have not established a consistent developmental perspective. There are contrasting views on whether children at early stages of development demonstrate more, less, or about the same amount of anticipatory coarticulation than adults. This study will focus on describing the patterns of anticipatory lingual coarticulation in the obstruent productions of three groups of children between 3 and 6 years of age $(N=42)$ and one comparison group of adults $(N=14)$. Frequency measures for the first and second formants were extracted from multiple tokens of a centralized and unstressed vowel (schwa) spoken prior to two different sets of productions. The first set of productions consisted of the target vowel followed by a series of real words containing an initial $\mathrm{CV}(\mathrm{C})$ syllable, while the second set consisted of nonword productions with a relatively constrained phonetic context. Formant values were transformed to a perceptually normalized scale (ERB) prior to statistical analysis. Analysis of variance was utilized to determine if the formant frequencies varied systematically as a function of age, gender, and phonetic context. [Work supported by research funding from Brigham Young University.]

2pSCa4. Developmental vowel acquisition data obtained by a bandfiltering analysis method: Results from Hungarian children. Krisztina Zajdó (Div. of Commun. Disord., Univ. of Wyoming, Dept. 3311, 1000 E. Univ. Ave., Laramie, WY 82071, kzajdo@uwyo.edu)

A recently developed band-filtering analysis method (van der Stelt et al., 2003, 2005; Zajdó et al., 2004) that minimizes the dependence of the results on fundamental frequency allows for a dependable identification of spectral measures in children's vowels. In the scripts, selection of segments with certain acoustic features (e.g., sound quality, F0) is followed by the calculation of spectral envelopes between $0-7 \mathrm{kHz}$, resulting in 40 filter values per spectrum. An intensity matrix is produced with data from each 40 filters. Data reduction is achieved through prinicipal component analysis. Results indicate that the first two principal components are related to the $F 1$ and $F 2$ of vowels, both in adults (Pols, 1977) and children (Zajdó et al., 2005). Analyses of unlabeled vowel productions in Hungarian-speaking children at 2;0,3;0 and 4;0 years show that the first two eigenvectors account for $>50 \%$ of the variability in all age groups. By using these eigenvectors, age-specific reference planes are created. Mapping phonemically labeled vowel measures onto the 4; 0-year-old's plane from each age group suggests that children produce decreasingly overlapping vowel categories as they age. With development, vowel categories are increasingly specified, covering more distinct areas of the acoustic vowel space. [Work supported by UW.]

2pSCa5. The acquisition of speech rhythm by bilingual Spanish- and English-speaking four- and five-year-old children. Ferenc Bunta (Dept. of Commun. Sci., Temple Univ., Weiss Hall, 1701 North 13th St., Philadelphia, PA 19122, fbunta@temple.edu) and David Ingram (Arizona State Univ., Tempe, AZ 85287)

This study investigates the acquisition of speech rhythm by bilingual Spanish- and English-speaking children, comparing their performance to that of monolingual age-matched peers in both languages, and to monolingual and bilingual adults. Participants included younger children (be- tween 3 years, 9 months and 4 years, 5 months, 15 days), older children (between 4 years, 6 months, 18 days and 5 years, 2 months), and adults (over 18 years). A sentence elicitation task of 26 sentences per language was used to obtain 64 samples that yielded over 15000 duration measurements of vocalic and intervocalic intervals. The duration measurements were entered into the normalized vocalic and intervocalic pairwise variability index (PVI) measures based on Grabe and Low (2002). The results indicated that younger bilingual children displayed distinct speech rhythm patterns for their target languages and deviated from their monolingual English-speaking peers. Older bilingual children also produced significant separation of speech rhythm, and differences between older bilingual children and their monolingual peers speaking English were also found. Comparisons between younger and older bilingual children indicated an effect for the vocalic PVI measure, but not for the intervocalic PVI, providing partial support for age differences for bilingual children. Bilingual adults showed separation of their languages and performed similarly to their monolingual peers, overall.

2pSCa6. Comparative study of perception of Japanese dialectal accent in children. Ayako Shirose (Waseda Univ., Mikajima 257915, Tokorozawa 3591129, Japan) and Kazuhiko Kakehi (Chukyo Univ., Tokodachi 101, Toyota 4700393, Japan)

This paper reports the acquisition course of perception of Japanese dialectal accent. We carried out an experiment of accent perception for native speakers of Japanese dialects, namely, Tokyo dialect (Standard Japanese) and Kagoshima dialect. Then we compared results between the two dialects. Experimental stimuli were real words with several types of synthesized accent patterns. Results revealed that the Kagoshima children and adults accepted both the native Kagoshima accent and the Tokyo accent, whereas the Tokyo children and adults accepted only the native Tokyo accent. These results suggest that the Kagoshima speakers had insensitivity to the perception of accent. In our previous studies, the Kagoshima children showed different characteristics of accent production from the Tokyo and Kyoto children: the Tokyo and Kyoto children acquired their native accent correctly, whereas the Kagoshima children failed to produce it. [Shirose et al., J. Cognit. Sci. 2(1), 33-44 (2001)]. The insensitivity of accent perception of both Kagoshima adults and children might be one factor contributing to results of accent production. These intriguing parallels of accent perception and production will be discussed inclusively.

2pSCa7. Infants' attention to repeated speech and musical patterns. Gerald W. McRoberts (Haskins Labs., 300 George St., Ste. 900, New Haven, CT 06511, mcroberts@haskins.yale.edu) and Colin Dawson (Dept. of Psych., 1503 E Univ. Blvd., Univ. of Arizona, Tucson, AZ 85721)

Previous investigations have shown that infants older than 5 months listen longer to speech with repeated utterances than to speech without utterance repetition. The present studies investigated whether infants also attend preferentially to repeated musical patterns. Musical phrases of four and five notes and ending with a rest were composed using several pitch contours and rhythmic patterns. Experiments 1 and 2 compared trials in which phrases were immediately repeated (AABBCC), to trials in which the same phrases occurred without repetition $(\mathrm{ABCDE})$. In the first experiment, no listening preference for either trial type was found for 6- or 9-month-old infants. In experiment 2 , silence was added after each phrase to assist infants in parsing the phrases. Infants again failed to show a listening preference. In experiment 3, trials of speech, half with repeated utterances and half without repeated utterances (from previous experiments), were interspersed with trials of repeated and nonrepeated music. Nine-month-olds demonstrated a preference for the repeated trials of both speech and music over nonrepeated trials of the same type. The fact that a preference for musical repetition is only obtained through induction (from verbal repetition) suggests that repeated speech may hold a special attentional status for infants. [Work was supported by Grants NIH_R15/ DC005947 and NIH_R01/DC00403.] 
2pSCa8. Infants listening to songs: Exploring a processing trade-off between phonetic and pitch patterns. Gina C. Cardillo and Patricia K. Kuhl (Inst. for Learning and Brain Sci., Box 357988, Univ. of Washington, Seattle, WA 98195, ginacc@u.washington.edu)

This investigation explored 11-month-olds' responses to changes in the phonetic (lyric) and pitch (melody) content of songs. Sixty infants were tested using a between-subjects auditory preference paradigm. After being familiarized with a four-note song, infants were presented with both the familiar song and a new song that contained either a novel syllable or pitch order. In experiment 1, infants preferred listening to a novel melody when the phonetic content was redundant, but showed no preference when the identical melody change occurred in the presence of variegated phonetic content. In experiment 2, we tested the hypothesis that infants presented with songs containing variegated phonetic information would attend to changes in words but not melodies. Infants marginally preferred listening to novel songs when only the lyrics changed, but did not show a preference when only the melodies changed. Taken together, the results are consistent with a priori predictions, and suggest that even though infants can recognize melodic changes when they are presented in isolation, identical changes become more difficult to detect when more complex phonetic information is introduced. Thus, neural resources at this age may be more heavily committed to processing phonetic information than pitch information when both are present. [Work supported by the J.S.McDonnell Foundation, NIH.]

2pSCa9. Cognitive influences in infant speech perception. Barbara $\mathrm{T}$. Conboy, Jessica Sommerville, Josie Randles, Caryn Deskines, and Patricia K. Kuhl (Inst. for Learning and Brain Sci., Univ. of Washington, UW Box 357988, Seattle, WA 98195-7988)

Speech perception during the first year reflects increasing attunement to native language phonetic features, but the mechanisms underlying this development are not well understood. Reductions in non-native phonetic discrimination have been linked to improvement in native phonetic discrimination and later vocabulary growth (Kuhl et al., 2005), and performance on nonlinguistic tasks (Lalonde and Werker, 1995). The present study examined links between native and non-native voice onset time discrimination, receptive vocabulary (MacArthur-Bates CDI), and cognitive control abilities at 11 months. Infants $(n=18)$ completed a doubletarget conditioned head turn task and two nonlinguistic tasks requiring attentional control and resistance to irrelevant cues (means-end and detour-reaching object retrieval). Infants with CDI scores above the median showed higher native discrimination scores, $t(16)=2.150 .05$, but no group differences for the nonnative contrast. Infants with scores above the median on either cognitive task showed worse discrimination of the nonnative contrast (means-end, $t(15)=2.27 p 0.04$; detour-reaching, $t(15)$ $=3.49, p .01$ ), but no group differences for the native contrast. These results suggest that cognitive control plays a role in infants' ability to ignore acoustic cues that are irrelevant to their native languages phonemic categories. [Work supported by NICHD (Grant HD37954) and a UW NSF Science of Learning Center Grant (LIFE).]

2pSCa10. Distinguishing voices: Are infants' abilities affected by age or language experience? Megha Sundara and Patricia K. Kuhl (Inst. for Learning \& Brain Sci., Univ. of Washington, Box 357988, Seattle, WA 98195-7988)

We know surprisingly little about infants' ability to encode voice information when listening to speech. Research with infants has focused on their ability to recognize their mother or fathers' voice and distinguish it from an unfamiliar voice. However, communication consists of interactions with unfamiliar speakers as well. Adults are well able to extract information about the speakers gender, size, age, dialect, etc., even from short samples of an unfamiliar persons voice. In experiment 1 , we tested 4- and 12-month-old English-learning infants on their ability to distinguish an unfamiliar male and female voice when listening to Hindi monosyllabic words. Results indicate that the 12-, but not the 4-month-olds can distin- guish an unfamiliar male and female voice when listening to Hindi words. In experiment 2, we tested 4- and 12-month-old English-learning infants on their ability to distinguish an unfamiliar male and female voice when listening to English monosyllabic words. Now, both 12- and 4-month-olds are able to distinguish the voices. Together, these results indicate that infants' ability to distinguish voices is affected by age as well as their language experience. We are now testing adults to determine if the effects of language experience on the ability to distinguish voices persists even after development.

2pSCa11. The bilingual brain: A comparison of native and non-native speech perception in monolingual and bilingual infants. Patricia $\mathrm{K}$. Kuhl, Sharon Coffey-Corina, Denise Padden, and Maritza Rivera-Gaxiola (Inst. for Learning \& Brain Sci., Univ. of Washington, Seattle, WA 98195, pkkuhl@u.washington.edu)

Infants raised in monolingual families are equally good at native and non-native speech discrimination early in life. By 12 months, performance on native speech has significantly improved while non-native performance declines. We tested bilingual American infants at 7 and 11 months of age on native (/ta-pa/) and non-native (Mandarin affricate-fricative) contrasts used in the monolingual tests. Phonetic discrimination was assessed using behavioral (conditioned head-turn) and brain (event-related potential) measures. The MacArthur Communicative Development Inventory estimated infants developing language skills at 14, 18, 24, and 30 months of age. The monolingual data [Kuhl et al., Language Learning and Development (2005)] demonstrated that at 7 months of age, infants native and non-native speech perception skills predicted their later language development, but differentially. Better native phonetic perception predicted more rapid language development between 14 and 30 months, whereas better non-native phonetic perception predicted slower language development over the same time period. Performance of bilingual infants on the same speech perception tasks, and their patterns of language growth between 14 and 30 months, will be compared to those of the monolingual infants, and a model that accounts for the results will be described. [Work supported by NSF, NIH, and the Human Frontiers Science Program.]

2pSCa12. Aussie, Aussie, Aussie, Oi-Oi-Oi: Infants love an Australian accent. Christine Kitamura (MARCS Auditory Labs., Univ. of Western Sydney, Locked Bag 1797, Penrith South DC NSW 1797, Australia, c.kitamura@uws.edu.au), Robin Panneton (Virginia Tech, Blacksburgm, VA 24061-0436), Anna Notley, and Catherine Best (Univ. of Western Sydney, Penrith South DC NSW 1797, Australia)

This study examines infant's attention to dialect differences following Nazzi et al.'s [J. Mem. Lang., 43, 1-19 (2000)] finding that American 5-month-olds can discriminate British- and American-English. Using a serial preference task, 48 6-month-old Australian and American infants heard sentence sets spoken in Australian- and American-English. Results showed that at 6 months, American infants listen longer to Australian than American sentences, but Australian infants show no preference. By 8 months, American infants also show no preference. The developmental lag suggests Australian infants have more exposure to the American dialect (e.g., television programs) than American infants to the Australian dialect. Thus, it is predicted, with less linguistic experience, Australian 3-montholds will show a dialect preference comparable to American 6-month-olds. Data from 14 3-month-olds support this hypothesis; moreover, they also listen longer to Australian than American sentences. Together the results imply that the ability to generalize across two dialects is a function of experience, and that, with age, infants filter out irrelevant phonetic information and cluster American and Australian dialects into the same group. Future directions of this study are discussed as are the reasons why Australian English is the dialect of preference for infants from both dialect environments. 
2pSCa13. Word extraction in adult-directed and infant-directed speech in African-American-English-learning infants. Shiri KatzGershon and Jean Andruski (Dept. of Audiol. Speech Lang. Pathol., Wayne State Univ., 207 Rackham, 60 Farnsworth St., Detroit, MI 48202)

Although there is evidence that infant-directed speech (IDS) facilitates word extraction in Standard-American-English (SAE)-learning infants, this effect has not been tested in African-American-English (AAE)learning infants. An important question that remains unaddressed is whether infants are better tuned to word boundary cues in their native dialect than a non-native dialect. Specifically, do infants extract words more efficiently from IDS in their native dialect than from IDS in a nonnative dialect. This study compares the ability of eight-month-old AAElearning infants to extract words from AAE and SAE IDS. The head-turn preference procedure was used to measure infants' memory for nonsense words embedded in sentences in AAE versus SAE. Infants were familiarized for 2 min with six sentences containing nonsense words and were then presented with both familiar and unfamiliar nonsense words carrying matching prosodic features. The difference in looking times between the familiar and unfamiliar nonsense words was used as a measure of word extraction. Results so far demonstrate an advantage of IDS over adultdirected speech in SAE. IDS in AAE is less advantageous than IDS in SAE. Acoustic analysis shows that the prosodic features of target nonsense words in SAE may make them more salient to infants than target words in the AAE sample.

2pSCa14. Exploring and learning the consequences of vocalizations in early infancy. Francisco Lacerda and Lisa Gustavsson (Dept. of Linguist. Stockholm Univ., SE-106 91 Stockholm, Sweden)

The ability to discover and learn about the consequences of own vocalizations is currently being studied in three groups of infants, 3-5-, 6-8-, and 9-11-month olds, exploring a situation that resembles an audiovisual memory game. The infants are tested individually, in a quiet and dimmed studio. They sit in front of a black computer monitor connected to an eye-tracking system. At randomly selected intervals, a circle appears in the center of the screen and expands and shrinks rapidly to a bright circle to signal that an observation trial is in progress. If the infant vocalizes during the trial, an animated actor emerges after about 200-ms delay at screen coordinates that are contingent on spectral characteristics of the initial part of the infant's vocalization. The actors brightness and time on display is controlled by the vocalizations overall intensity. By analyzing the changes in predictive looking behavior that probably occur during the session, we expect to gather valuable information on the infant's capacity to learn about consequences of their vocalizations at different ages. [Work supported by grants from the Bank of Sweden Tercentenary Foundation (MILLE, K2003-0867) and EU NEST program (CONTACT, 5010).]
2pSCa15. The acoustic characteristics of infant cries. Naoto Yamane and Yoko Shimura (The United Grad. School of Education, Tokyo Gakugei Univ., 4-1-1 Nukuikitamachi, Koganei, Tokyo, 184-8501, Japan, r052004s@u-gakugei.ac.jp)

The present study measured the acoustic characteristics of infant cries. Twenty-eight infants participated, ranging from 2 to 23 months of age. Crying was elicited when their mother moved to a distance, leaving them in the lap of unknown person. The cries were recorded with a microphone placed $1 \mathrm{~m}$ from the infant's mouth in an anechoic room. Analysis of the recorded cries revealed four patterns: cries were classified as either voiced or breathy, and as higher or lower than $7 \mathrm{kHz}$. Certain cry characteristics were later rated as more stress-causing, suggesting they could potentially elicit more abuse reactions. The recorded cries were presented to adults and breathy cries with a high-frequency component were labeled as emergency cries and as likely to cause high stress levels in parents and caregivers. In Japan, living spaces have recently begun changing such that environments are more echoic, perhaps exacerbating negative reactions to high-frequency components of infant cries.

2pSCa16. Discovering place and manner features-What can be learned from acoustic and articulatory data? Ying Lin (Dept. of Linguist, Univ. of Arizona, Tucson, AZ 85721) and Jeff Mielke (Univ. of Ottawa, Ottawa)

Theories of phonological acquisition often assume that features are innate and universal, and establishing distinctive features requires the availability of minimal pairs. In this study, we explore an inductive approach to feature discovery without resorting to minimal pairs. Our basic assumption is that learners have access to a wide variety of phonetic information, and so feature induction can proceed through recursive clustering of the data. To test our hypothesis, we used as our data acoustic and articulatory measurements made simultaneously from a set of CVC syllables. The articulatory data are obtained with ultrasound imaging, and the vocal tract is characterized by a vector of cross distances from the tongue body to a reconstructed palate. The raw articulatory data are then mapped to a lower dimensional space using principle components, on which clustering is carried out. Experiments using acoustic and articulatory data suggest that these two sources of information lead to the discovery of place and manner features, respectively, thus bringing support to an alternative theory of feature learning. 


\title{
Session 2pSCb
}

\section{Speech Communication and Psychological and Physiological Acoustics: Second Language Acquisition I}

\author{
Jared C. Bernstein, Cochair \\ Ordinate Corporation, 800 El Camino Real, Ste. 400, Menlo Park, CA 94025 \\ Reiko Akahane-Yamada, Cochair \\ ATR, 2-2-2 Hikaridai, Seika-cho, Soraku-gun, Kyoto 619-0288, Japan
}

Chair's Introduction-2:15

Invited Papers

2:20

2pSCb1. Second-language speech perception: The modification of automatic selective perceptual routines. Winifred Strange (Ph.D. Program in Speech and Hearing Sci., City Univ. of New York Grad. School, 365 Fifth Ave., New York, NY 10016, strangepin@aol.com)

It is well established that late second language (L2) learners show significant difficulties in categorizing L2 consonants and vowels that do not occur in the native language (L1), or that occur, but differ in their phonetic realization in L1 and L2. These perceptual problems underlie (some) L2 production difficulties (accented L2 speech); lead to L2 comprehension difficulties, especially in nonoptimal listening environments; and may persist even after years of immersion in the L2 environment. However, it has been shown that intensive laboratory training (listening with feedback) can lead to improved perception of difficult non-native contrasts that: (a) generalizes to novel speakers and stimulus materials, (b) persists over months, and (c) produces improvements in production of trained segments. In the model presented here, changes with experience in patterns of phonetic perception are conceived of as reflecting the modification of language-specific (L1) selective-perceptual routines used to extract phonologically relevant information efficiently from continuous speech input. Theoretical and methodological issues will be discussed regarding (a) the conceptualization of (changes in) the structure of phonetic/phonological categories, (b) empirical assessment of phonetic perceptual learning, (c) operational definitions of mastery of L2 phonological categorization, and (d) implications for improving phonetic perceptual training techniques. [Work supported by NIDCD.]

2:40

2pSCb2. STRAIGHT as a research tool for L2 study: How to manipulate segmental and supra-segmental features. Hideki Kawahara (Faculty of Systems Eng., Wakayama Univ., 930 Sakaedani, Wakayama 640-8510, Japan) and Reiko Akahane-Yamada (ATR, Sorakugun, Kyoto 619-0288, Japan)

A morphing algorithm based on STRAIGHT speech manipulation system [Kawahara et. al., ICASSP (2003)] has a potential importance in L2 study, since it generates stimulus continuum with quality close to natural speech. The morphing procedure yields an equally spaced continuum in an abstract parameter domain, which is spanned by fundamental frequency, STRAIGHT's timefrequency representation (a variant of smoothed spectrogram), the periodicity map, and the time-frequency coordinate. Instead of manipulating all these parameters at once, it is useful to manipulate each parameter independently. This individual parameter manipulation can also be generalized to coupled manipulation by introducing new parameters defined using a set of parameters. This extension enables us to manipulate segmental and supra-segmental features instead of manipulating abstract physical parameters. For example, coupling energy envelope, fundamental frequency, and temporal axis stretching factor yield access to a prosodic feature that is basically supra-segmental. Manipulation of spectral landmark positions yields access to a phonological feature that is basically segmental. A preliminary test results using the right-light continuum sets generated using a STRAIGHT-based method and the Klatt synthesizer will also be presented.

3:00

2pSCb3. Structural representation of the pronunciation and its application to computer-aided language learning. Nobuaki Minematsu (Grad. School of Frontier Sci., The Univ. of Tokyo, 5-1-5, Kashiwanowa, Kashiwa-shi, Chiba, 277-8562, Japan)

The conventional speech applications have been developed by looking at sound substances of speech and this strategy is derived from acoustic phonetics. However, the speech representation of this framework, e.g., spectrogram, is inevitably with nonlinguistic factors such as speakers and microphones. Then, all the developed systems come to select users, where the systems may work fine with a major part of the users but still work poorly with the others. The author believes that this situation should be avoided for educational applications because users don't know whether they are outliers or not. Recently, the author proposed another framework of developing speech applications by looking at only sound contrasts and this strategy is derived from physically and mathematically interpreting structural phonology. On this new framework, nonlinguistic factors such as speakers and microphones are mathematically 
removed from speech as pitch information can be removed by smoothing the spectrogram. This talk shows what is possible by the new framework, which completely discards the absolute acoustic properties such as formants and spectral envelopes. In other words, in the new framework, an utterance is modeled as organized pattern of sounds although it has been modeled just as a linear string of sounds in the conventional framework.

$3: 20$

2pSCb4. Using speech recognition for just-in-time language learning. Maxine Eskenazi, Antoine Raux, and Elizabeth Harris (Carnegie Mellon Univ., 4619 Newell Simon Hall, 5000 Forbes Ave., Pittsburgh, PA 15213)

While many language learning systems rely on the student to be a dedicated learner, the opportunity to teach language is present outside the classic teaching situation, in all everyday oral and text-related activities. Teaching language in real situations, where the new knowledge is needed and used immediately, has great potential for robust learning: items are retained over a long period of time, make learning other items easier, and are generalized to other situations. Giving immediate and effective error detection and corrective feedback is a technical challenge for language modeling, spoken dialogue structure, and targeted speech synthesis. We will discuss just-in-time language learning and then present one type of just-in-time learning, lexical entrainment, where the automatic system detects an oral error and then respeaks the utterance that contained the error in corrected form, emphasizing the corrected portion. To test the effectiveness of this approach, the Lets Go spoken dialogue system that furnishes bus information to Pittsburghers was modified to predict errors from a variety of non-native speakers of English. A study of the effectiveness of lexical entrainment for a group of non-native users will be described. [This work is sponsored by NSF Grants 0208835 and IIS0096139.]

\title{
$3: 40$
}

2pSCb5. Theory and method in automatic evaluation of spoken language proficiency. Jared Bernstein (Ordinate Corp., $800 \mathrm{El}$ Camino Real, Menlo Park, CA 94025)

Several kinds of theory (linguistic, psycholinguistic, and sociolinguistic) have been used to justify different methods of language proficiency assessment or to explain patterns of performance observed in proficiency tests. After reviewing some traditional methods of testing spoken language performance (e.g., structured interviews, listening comprehension, word identification in noise, and elicited imitation), the assumptions underlying these methods are compared. When the practicality, reliability, and validity of assessment methods are evaluated, differences in the associated theories become clearer. A low perplexity testing method is presented that scores a set of context-free speech-interactive tasks using speech recognition and analysis techniques to measure aspects of the linguistic and phonetic content of spoken responses. Data from several experiments comparing interview testing with low-perplexity testing on populations of second language speakers (of English, Spanish, and Dutch) suggest that nearly all the information that expert judges extract about levels of communicative competence can be accounted for in terms of automaticity of spoken language processing. Furthermore, measurement techniques that are feasible in low-perplexity testing provide much greater precision in the spoken language proficiency reporting scale than was previously feasible, opening up a range of new applications.

WEDNESDAY AFTERNOON, 29 NOVEMBER 2006

HONOLULU ROOM, 1:00 TO 5:00 P.M.

\section{Session 2pSP}

\section{Signal Processing in Acoustics: Spatial Speech, Non-Destructive, Time Reversal}

\author{
Elizabeth A. McLaughlin, Cochair \\ Naval Undersea Warfare Center, 1176 Howell St., Newport, RI 02841 \\ Yoshifumi Chisaki, Cochair \\ Kumamoto Univ., Faculty of Engineering, 2-39-1 Kurokami, Kumamoto 860-8555, Japan
}

Contributed Papers

1:00

2pSP1. Evaluation of spatial impression of sound in horizontal and depth directions for the purpose of developing an advanced multichannel live sound reproduction system. Hiroyuki Okubo, Toshiyuki Nishiguchi, Reiko Okumura, and Kimio Hamasaki (Human \& Information Sci., NHK Sci. \& Tech. Res. Labs., 1-10-11 Kinuta Setagaya, Tokyo 157-8510 Japan)

A prototype of an advanced multichannel live sound reproduction system was developed. In this system, multiple loudspeakers are arranged in the horizontal and depth directions in front of listeners to reproduce a sense of sound depth and width [K. Hamasaki et al., Proc. 119th AES Convention, (2005) p. 6559 ]. Using this prototype, a number of subjective tests were conducted to evaluate the listeners sense of sound depth and width. The results revealed that while the direct sound source was perceived near the predicted horizontal location, the sense of depth varied largely from subject to subject. Another listening test was conducted in which reflections from the upper-front of the listeners were reproduced. Simulated reflections of a virtual space were divided into 11 directional room impulse responses (RIRs). The RIRs were convoluted with individual sound sources (male vocals), and subjects compared their spatial impressions of the sound sources reproduced with and without such reflections. The results of this listening test revealed that the feeling of sound depth was one of the factors of spatial impressions and it increased when the reflections from the upper-frontal direction were reproduced. 
2pSP2. Precise position measurement of objects using a coded acoustic signal in an indoor environment. Kuramitsu Nishihara (Grad. School of Sci. and Technol., Chiba Univ., 1-33 Yayoi-cho Inage-ku, Chiba, 263-8522 Japan), Tadashi Yamaguchi, and Hiroyuki Hachiya (Chiba Univ., Chiba, Japan)

Acoustic sensing in the air might serve as an effective technique for acquiring information about an object, such as its distance, shape, and surface configuration. Nevertheless, it is difficult to identify acoustic waves clearly because many mirror reflections or environmental noises exist in the air. In addition, acoustic measurement requires a longer time than measurements using lasers or electromagnetic waves because sound propagates much more slowly than light. For this study, we attempted highly precise position measurement using an $M$-sequence signal in an indoor environment. The degrees of $M$-sequence were 7-14; the transmitting frequency was $25 \mathrm{kHz}$. A signal reflected from various angles was received by moving the receiving unit, which contained three microphones. Measurement results with the $M$-sequence signal showed that the signal-to-noise ratio $(\mathrm{S} / \mathrm{N})$ improved $20-40 \mathrm{~dB}$ compared with a burst wave. Furthermore, the distance resolution improved when the $M$-sequence degree was changed. The distance and the angle of multiple objects installed indoors or in walls were detectable as a result of construction of a two-dimensional plane based on received signals.

\section{1:30}

2pSP3. Self-similar analysis of the room impulse response. Yong Tang, Hideo Shibayam, Wakako Tanaka, and Tooru Itakura (Dept. of Commun. Eng., Shibaura Inst. of Technol., 3-7-5 Toyosu Koutou-ku, Tokyo, 135-8548 Japan,m603101@ sic.shibaura-it.ac.jp)

Important information of the room acoustic characteristics is contained in the room impulse response. We analyze the time-space correlation of the impulse response by the self-similarity method. The room impulse response is divided into the frame of the length of the fixed time. Attractors are composed by affine transformation of each frame. The attractors that make the Hausdorff distance become the shortest between the standard frame and attractors are decided. The impulse response is reconstructed by using these attractors. In the self-similar analysis, the frame length is decided from the period of the Hausdorff distance between the initial sound and the reflection sound. We show results for the relation between the room acoustic characteristics and the attractors calculated from the self-similar analysis.

\section{1:45}

2pSP4. Application of dynamic synapse neural networks on gunshot recognition. Alireza A. Dibazar, Hyung Ook Park, Walter M. Yamada, and Theodore W. Berger (Dept. of Biomed. Eng., Univ. of Southern California, 1042 Downey Way, DRB140, Los Angeles, CA 90089-1111, dibazar@usc.edu)

Identification of acoustic signals in noisy environments remains one of the most difficult of signal processing problems, and is a major obstacle to the high degree of accuracy and speed needed to identify suspicious sounds in high-security, high-safety environments. We have previously developed an acoustic recognition capability using a novel, biologically based Dynamic Synapse Neural Network (DSNN) technology. The DSNN-based technology has been demonstrated to classify target sounds with a high degree of accuracy, even in high noise conditions. In this paper we focus on extending the acoustic recognition capability of DSNNs to the problem of gunshot recognition. In order to recognize and localize the event, an array of four microphones is used for sound input. For localization purpose, time-delay estimation algorithm (TDE) is employed for triangulation. We have developed stand-alone, portable, and cost-efficient hardware with which both recognition and localization can be performed. In field-testing, the system classifies and localizes over $90 \%$ of the trainedfor sounds. Performance for firecracker, starter pistol, 9-mm, and 44caliber, explosion/firing sounds was also tested.
2pSP5. Effects of reproduction methods and audio coding on word intelligibility with competing talkers. Kenji Ozawa and Junichi Nakagai (Interdisciplinary Grad. Sch. of Med. and Eng., Univ. of Yamanashi, 4-3-11 Takeda, Kofu, 400-8511 Japan, ozawa@yamanashi.ac.jp)

Effects of reproduction methods and high-efficiency audio coding on word intelligibility were examined in a tele-collaboration system. Monaural, stereophonic, and binaural methods were studied. Transform domain weighted interleave vector quantization (TwinVQ) [Iwakami et al., Proc. ICASSP'95, pp. 937-940 (1995)] was used as the audio coding method. Intelligibility tests using word lists, in which word familiarity was controlled, were conducted under six conditions combining three reproduction methods with two conditions, i.e., with or without audio coding. In the experiments, three talkers pronounced different words simultaneously; a listener was then asked to selectively hear the word of a designated talker. Analyses of variance of the experimental results showed that intelligibility for the binaural method was higher than for the other two reproduction methods in both conditions with or without audio coding. Moreover, the benefit of the binaural method was demonstrated because intelligibility for the method with audio coding was higher than that for the stereophonic method without coding, whereas the amount of audio data in the former method was much smaller than in the latter, indicating that the binaural method with high-efficiency audio coding is useful for a tele-collaboration system. [Work supported by JSPS: KAKENHI (17560336) and RIEC, Tohoku Univ. (H18/A10).]

\section{2:15}

2pSP6. Environmental adaptation of hidden Markov models in an automatic sounds recognition system used for surveillance applications. Asma Rabaoui, Zied Lachiri, and Noureddine Ellouze (ENIT (Ecole Nationale des Ingenieurs de Tunis) Rue Ibn Mandhour, Residence Sana, Bloc Yasmine, App10, ARIANA 2080 Tunisie)

Generally, the main goal of an automatic sound recognition system is to analyze in real time the sound environment of a habitat and to detect the abnormal sounds that could indicate a distress situation. In a surveillance application, the most encountered problem is the background noise that can be captured with the sounds to be identified. Thus, we propose to use a multistyle training system based on HMMs: one recognizer is trained on a database including different levels of background noises and is used as a universal recognizer for every environment. In order to enhance the system robustness, we will explore different adaptation algorithms that reduce the environmental variability. The algorithms closely examined are maximum likelihood linear regression (MLLR), maximum a posteriori (MAP), and the MAP/MLLR algorithm that combines MAP and MLLR. Experimental evaluation illustrates a recognition improvement over the baseline system (i.e., none adapted system) results. By using 156 adaptation scenes, the error rate of the adapted recognizer improves over the baseline system by more than $30 \%$ for rather all the considered algorithms, even under important noise degradation conditions.

\section{$2: 30$}

2pSP7. Passive acoustic fiber Bragg grating sensor measurements of Green function in an instrumented part. Karl A. Fisher, Sean K. Lehman, and David H. Chambers (Lawrence Livermore Natl. Lab., L-333, 7000 East Ave., Livermore, CA 94550)

The development of fiber Bragg grating (FBG) sensors for acoustic measurements permits the instrumentation of manufactured composite parts for the purposes of nondestructive evaluation (NDE) and lifetime monitoring. The parts can be designed from conception with an array of FBG acoustic receivers integrated into its internal structure for the purpose of multistatic data collection for NDE and monitoring of internal changes. The FBG array is accessed via a port on the outer surface and requires no additional power demands on the operation of the part. Data collection is accomplished by activating sources on the surface of the part and measuring the fields along the FBG array. We are particularly interested in Green function (GF) estimation of a FBG instrumented composite structure. By 
passively measuring diffuse acoustic fields throughout the volume, we use a technique developed by Lobkis and Weaver [J. Acoust. Soc. Am. 110(6), 3011-3017 (2001)] to estimate the GF within the part and monitor it over time. We describe FBG sensor array operation, review the theory of diffuse field GF estimation, and present examples in heterogeneous multilayered structures. [This work was performed under the auspices of the U.S. DOE by University of California Lawrence Livermore National Laboratory under Contract No. W-7405-Eng-48.]

\section{$2: 45$}

2pSP8. Super-resolution algorithms for ultrasonic nondestructive evaluation imagery. Grace Clark (Univ. of California Lawrence Livermore Natl. Lab., 7000 East Ave., L-130, Livermore, CA 94550, clark9@1lnl.gov) and Jessie Jackson (Univ. of California Lawrence Livermore Natl. Lab., Livermore, CA 94550)

The spatio-temporal range resolution of ultrasonic measurements is often severely limited by the inherent band-limited spectral transfer function of ultrasonic transducers. This is manifested as ringing in the time domain. Super-resolution algorithms and a new MATLAB-based software system are presented for improving spatial resolution in one-dimensional (1-D) pulse-echo signals (A-scans). Two-dimensional (2-D) images (Bscans) and 3-D volumes are formed from suites of processed 1-D signals. The algorithms solve two inverse problems that are ill-posed and illconditioned. Regularization techniques and prior constraints are used to ensure useful results in practice. Given a noisy reflection signal (A-scan) $u(t)$ and an associated reference signal $x(t)$, the algorithms first solve the system identification problem to produce an optimal least-squares bandlimited estimate $\hat{h}(t)$ of the impulse response of the material under test. Next, an algorithm based upon the method of alternating orthogonal projections produces a spectrum-extrapolated version $\hat{h}_{e}(t)$ of the impulse response estimate. This estimate, when used in place of the raw reflection signal $u(t)$, enhances the spatio-temporal resolution by mitigating the transducer distortion. The efficacy of the algorithms is demonstrated on real data from controlled experiments. Three different practical applications are presented, showing improved location of material interface boundaries. [Work supported by Grant UCRL-ABS-222494.]

\section{3:00-3:15 Break}

\section{$3: 15$}

2pSP9. Nondestructive technique to detect a crack in a concrete block by precise estimation of the surface wave. Takeshi Murakami, Toyota Fujioka, Yoshifumi Nagata, and Masato Abe (Dept. of Comput. and Information Sci., Faculty of Eng., Iwate Univ., Ueda 4-3-5, Morioka City, Iwate, Japan)

This paper describes a method to estimate a crack's position in a concrete block using several accelerometers. An array of accelerometers is attached to the concrete block and an impact is made on the concrete surface with a small hammer. If a crack exists, a reflection wave is generated at the crack position. Conventional methods to estimate the reflection point may seem to be useful for detection. Since the concrete block is elastic, however, it has three wave propagation modes: the surface wave mode, the primary wave mode, and the secondary wave mode. We cannot estimate the position using conventional methods because the necessary primary wave mode is weaker than the surface wave mode. To estimate the crack position precisely, we have already proposed two methods to eliminate the surface wave and side wall reflections. However, since the elimination is insufficient, they sometimes yield a peak at a position where there is no crack. Therefore, this paper proposes a method to estimate the surface wave more precisely to suppress such a peak. Some experiments were carried out, yielding better results.
3:30

2pSP10. Applying broadband beam-based decomposition of the time reversal operator (BBDORT) to midfrequency active sonar systems. David M. Fromm (Naval Res. Lab., 4555 Overlook Ave. SW, Washington, DC 20375-5350)

This paper reports on progress developing broadband beam-based decomposition of the time-reversal operator (BBDORT) for midfrequency hull-mounted active sonar systems. BBDORT combined with backpropagation has been previously demonstrated to localize a mid-water-column target in shallow water using a vertical source-receiver array [Fromm and Gaumond, J. Acous. Soc. Am. 118, 2039]. However, the array spanned the water column, and choosing an optimal beam set (to scan for targets in a short period of time) can make as much as 30-dB target improvement over nonoptimal beam sets. Current research efforts will address: sourcereceiver array configurations and tactical frequencies of Navy operational interest, evaluating performance in different environments, and determining efficient algorithms to optimize beam sets. [Work supported by the Office of Naval Research.]

\section{$3: 45$}

2pSP11. Properties of focused time reversal operator in random media. Jean-Luc Robert (Philips Res. North America, 345 Scarborough Rd., Briarcliff Manor, NY 10510, jean-luc.robert@philips.com) and Mathias Fink (Universite Paris 7, 75005 Paris, France)

FDORT (focused DORT) is an alternative of the DORT method using focused transmits instead of single-element transmits. In the case of wellresolved point scatterers, the FDORT eigenvectors may be understood as invariants of the time reversal operator. However, if the medium is a collection of many subresolution scatterers (speckle), a different interpretation is more helpful. It is shown that, in speckle, the FDORT matrix may be interpreted statistically as a spatial correlation matrix, whose coefficients are the correlation between signals received by pairs of elements. The matrix is fully described by the Van Cittert Zernike theorem. The first eigenvector still has focusing properties, and the eigenvalues can be related to the so-called focusing criterion. Applications to aberration estimation in medical ultrasound are presented.

\section{4:00}

2pSP12. Spatio-temporal invariants of the time-reversal operator. Jean-Luc Robert (Philips Res. North America, 345 Scarborough Rd., Briarcliff Manor, NY 10510, jean-luc.robert@philips.com) and Mathias Fink (Universite Paris7/ESPCI, 75005 Paris, France)

The DORT method gives the spatial invariants of the monochromatic time-reversal operator, at a given temporal frequency. It allows the monochromatic Green functions of point scatterers to be extracted from a data set. However, in acoustics, broadband signals are usually used and thus, it is often interesting to have the invariants in the time domain, or to know the evolution of the monochromatic Green function with frequency. It is not always easy, especially in complex media, because the sequence of the DORT eigenvectors (the invariants) changes as a function of the frequency. Thus, an invariant corresponding to a given scatterer may be the first at low frequencies, but the second at higher frequencies. Therefore, it is difficult to reconstruct a spatio-temporal Green function from monochromatic invariants. We introduce here the spatio-temporal time-reversal operator as a linear operator described by a fourth-order tensor, and deduce a method to obtain its spatio-temporal invariants. The method reduces to a more straightforward matrix decomposition, and thus can be easily implemented. Properties of the invariants are described, and applications to focusing in complex media are presented. 
position. This correlation often shows a multimodal structure due to the nonlinear parameter dependence: in addition to a mainlobe around the true source position, there are unpredictable prominent high sidelobes elsewhere. For a well-conditioned problem, in the absence of noise the peak output is guaranteed to occur at the true source position. In the presence of noise, we will probably have some peak outputs around the sidelobes, introducing a large localization error. Therefore, output ambiguity structure is a very important factor in the development of any matched-field algorithm operated in low-SNR scenarios. To analyze the ambiguity behavior, a quantitative approach for error analysis has previously been developed in the context of the maximum likelihood estimate (MLE) with spatially white noise. In this talk, generalization to including spatially correlated noise field introduced by discrete interferences and/or surface distribution sources is discussed.

\section{$4: 45$}

2pSP15. Multiuser communications using passive time reversal. Heechun Song, William S. Hodgkiss, William A. Kuperman, Tuncay Akal (MPL/SIO, UCSD, La Jolla, CA 92093-0238), and Mark Stevenson (NATO Undersea Res. Ctr., La Spezia, Italy)

A recent paper [Song et al., IEEE J. Ocean. Eng. 31, 165-173 (2006)] demonstrated multipleinput/multiple-output (MIMO) multi user communications in shallow water using active time reversal where the time reversal array (i.e., base station) sent different messages to multiple users simultaneously over a common bandwidth channel. Passive time reversal essentially is equivalent to active time reversal with the communications link being in the opposite direction. This paper describes MIMO passive time reversal communications, which enables multiple users to send information simultaneously to the time reversal array. Experimental results at 3.5 $\mathrm{kHz}$ with a $1-\mathrm{kHz}$ bandwidth demonstrate that as many as six users can transmit information over a 4-km range in 120-m-deep shallow water using QPSK modulation, achieving an aggregate data rate of $6 \mathrm{kbits} / \mathrm{s}$. Moreover, the same data rate has been achieved at $20 \mathrm{~km}$ range by three users using 16-QAM modulation.

\title{
Underwater Acoustics and Acoustical Oceanography: Sediment Acoustic Processes: Quantifying the Effects of Sediment Properties on Dispersion, Attenuation, Reflection, Scattering, and Buried Target Detection II
}

\author{
Eric I. Thorsos, Cochair
}

Univ. of Washington, Applied Physics Lab., 1013 NE 40th St., Seattle, WA 98105-6606

Masao Kimura, Cochair

Tokai Univ., Dept. of Geo-Environmental Technology, 3-20-1 Shimizu-Orido, Shizuoka 424-8610, Japan

\section{Contributed Papers}

1:00

2pUW1. Concerning the sediment volume contribution to scattering in SAX04 (Sediment Acoustics Experiment 2004) sediment: Sound speed and bulk density fluctuations. Kevin Briggs (Seafloor Sci. Branch, Naval Res. Lab., Stennis Space Ctr., MS 39529-5004)

Sound speed and bulk density profiles measured from SAX04 sediment cores are used to estimate correlation lengths of fluctuations in the sound speed and the bulk density. Sound speed is measured from 5.9-cmdiameter cores at $1-\mathrm{cm}$ intervals and bulk density is measured at $2-\mathrm{cm}$ intervals from the same cores. Previous estimates of these parameters from experimental data involved fewer realizations (cores), limited sampling areas, and samples without mud flasers within the sand sediment. Values of correlation length may vary according to location within the SAX04 site. Correlation length values estimated with Burg's algorithm from sediment sound-speed fluctuations averaged $2.24 \mathrm{~cm}$, from sediment bulk density fluctuations averaged $2.53 \mathrm{~cm}$. Ranges of values of correlation length estimated from bulk density fluctuations from locations inside the experiment site are used to present a range of volume scattering strength predictions, given the other parameters of sediment sound speed, sound attenuation, density ratio variance, and ratio of sound speed to density fluctuations used by the perturbation approximation to the composite roughness model for each location. At the SAX04 site, interface scattering 
dominated over volume scattering, despite the presence of mud inclusions with strong sound speed and bulk density contrasts. [Work sponsored by ONR.]

\section{1:15}

2pUW2. The effects of layering on reflection coefficient and transmission measurements from a sand/water interface at the Sediment Acoustics Experiment 2004 (SAX04). Marcia Isakson (Appl. Res. Labs., Univ. of Texas, Austin, TX 78713)

During the Sediment Acoustics Experiment 2004 (SAX04), transmission measurements from 4.5 to $50 \mathrm{kHz}$ were taken on an eight-transducer array buried at an angle of $15 \mathrm{deg}$ using a source mounted on a remotely operated vehicle (ROV). Simultaneously, the reflection coefficient was measured using a three-receiver vertical line array (VLA). Reflection coefficient measurements at angles from 10 to 70 deg were obtained. Environmental characterization of the area has revealed layering effects such as a surficial mud layer, mud inclusions, and depth-dependent sediment properties such as porosity. The consequence of this layering on the variability of the amplitude of reflection coefficient measurements and the time of arrival of transmission measurements will be investigated. [Work supported by ONR, Ocean Acoustics.]

\section{$1: 30$}

2pUW3. Measurement and modeling of elliptical particle motion in the seabed. John C. Osler, David M. F. Chapman, Paul C. Hines, Jeffrey Scrutton (DRDC Atlantic, P.O. Box 1012, Dartmouth, NS B2Y 3Z7, Canada, john.osler@drdc-rddc.gc.ca), and Anthony P. Lyons (The Penn State Univ., State College, PA 16804)

As part of the SAX04 experiment conducted off the coast of Florida in the Gulf of Mexico, four vector sensors containing three-axis accelerometers and pressure sensors were buried in the seabed. These served as the receivers to measure sediment sound-speed dispersion using a variety of techniques. One technique involved the generation of spherical waves from a point source in water and transmitted into the seabed in the frequency band 800 to $3000 \mathrm{~Hz}$. In this geometry, the contribution of inhomogeneous waves to the field results in elliptical-rather than longitudinal - particle motion. The orientation of the elliptical orbit varies with the source-receiver geometry, frequency, and the sediment sound speed, thereby allowing measurements of sediment sound-speed dispersion. However, the orientation of the ellipse major axis is not always aligned with the direction of wave propagation suggested by Snell's law. Measurements were made at several angles approaching the nominal critical angle in order to address the trade-off between increased sensitivity of the measurement versus greater departure from Snell's law. Measurements and modeling of the elliptical particle motion are compared to ensure that the sediment sound-speed estimates account for any bias created by the inhomogeneous waves. [Work partially supported by ONR.]

\section{1:45}

2pUW4. Characterization and modeling of synthetic aperture sonar image statistics. Anthony P. Lyons, Shawn F. Johnson, Douglas A. Abraham (The Penn State Univ., Appl. Res. Lab., State College, PA 16804, ap12@psu.edu), and Kevin L. Williams (Univ. of Washington, Seattle, WA 98105)

Interest in the application of synthetic aperture sonar (SAS) to seafloor mapping and object detection has been increasing in recent years. Because images of the seafloor obtained with SAS systems can sometimes exhibit large pixel-to-pixel fluctuations, robust seafloor segmentation or target detection still requires a thorough understanding of the SAS image statistics and their relation to seafloor parameters. As part of the SAX04 sediment acoustics experiment, acoustic data suitable for SAS processing were collected using a bottom-mounted rail system off the coast of Florida in the Gulf of Mexico in October, 2004. This unique data set has been used to explore several important aspects of SAS image statistics including their dependence on system resolution and on the correlation structure of SAS reverberation due to ripples that were present at the study site. Models of the reverberation envelope probability distribution function (pdf) based on the $K$-distribution have been found to well describe the reverberation environment for systems with lower resolutions than possible with broadband SAS systems. Characterization and modeling of SAS image statistics based on the $K$-distribution and using ground truth collected during SAX04 will be presented. [Work performed under ONR Grants N0001404-1-0013 and N00014-06-1-0245.]

\section{2:00}

2pUW5. Modeling the effect of multipath on high-resolution synthetic aperture sonar image statistics. Shawn F. Johnson, Anthony P. Lyons, Douglas A. Abraham (Appl. Res. Lab., The Penn State Univ., State College, PA 16804, sfjohnson@psu.edu), and Kevin Williams (Univ. of Washington, Seattle, WA 98105)

Recent analysis of Synthetic Aperture Sonar data collected during the Office of Naval Research sponsored SAX04 experiment in the Gulf of Mexico during October 2004 has shown that the processed image has varying amplitude statistics as a function of range. Since syntheticaperture sonar maintains a constant range-independent resolution cell size, unlike traditional sonar systems, one would expect that the statistics of the image would be based solely on the seafloor properties. The K-distribution shape parameter was estimated as a function of range and increases at discrete ranges corresponding to the onset of multi-path propagation (low shape parameter values represent a heavy tailed distribution). At these points of increase in shape parameter, scattered returns arising from additional propagation paths arrive with the result that two or more parts of the seafloor are contributing to the same sonar resolution cell, which leads to a larger K-distribution shape parameter than would be expected when only the direct path contributes. Model results using the sonar system geometry and interface scattering theory will be used to illustrate this effect. [Work supported by ONR Codes 321 and 333. First author supported by a National Defense Science and Engineering Graduate Fellowship.]

\section{2:15}

2pUW6. Synthetic aperture sonar imaging of buried spheres and cylinders. Steven G. Kargl and Kevin L. Williams (Appl. Phys. Lab., 1013 NE 40th St., Seattle, WA 98105)

During SAX04, synthetic aperture sonar (SAS) was used to detect buried spheres where the nominal grazing angle of incidence from the SAS to the point above a sphere was well below the sediment's critical grazing angle. SAS images from other measurements below the critical angle on buried spheres and finite cylinders in the NSWC-PC test pond facility have also produced target detections. Numerical simulations will be discussed that accurately predict the observed signal to noise (SNR). Simulations include estimates of reverberation from the rough seafloor, and the subcritical penetration through the seafloor, scattering from a target, and propagation back to the SAS. Required material parameters were obtained from environmental measurements including profiles of the small-scale surface roughness and the superimposed ripple structure. These simulations, the sonar equation results, and the model/measurement comparisons over the frequency range of $10-50 \mathrm{kHz}$ further support sediment ripple structure as the dominant mechanism for subcritical penetration in this range. [Work supported by ONR.]

$$
\text { 2:30 }
$$

2pUW7. Measurement of scattering wave from a buried object using an underwater vehicle. Koichiro Tanaka, Taku Suto, and Takahiro Fukai (Ishikawajima-Harima Heavy Industries Co., Ltd., 1, Shin-Nakahara-Cho, Isogo-Ku, Yokohama 235-8501, Japan, kouichirou_tanaka@ihi.co.jp)

Measurements of a scattering wave from a buried object were carried out at a site in Tokyo Bay. The buried object was set below the bottom with about 10-m water depth. A crane suspended a subbottom profiler to insonify the object. In these measurements, the medium frequency (less than $10 \mathrm{kHz}$ ) was used to penetrate near the critical angle. Also, when the object shape is a cylinder and so on, the scattering wave appears strongly 
in the specific direction, which is with the shape of the object and the physical relationship of the source and the object. The direction where this scattering wave appears is strongly different from the source position, and it is difficult to catch in the mono-static position. Especially, because the level of the scattering wave becomes low in the case of the scattering from the object under the seafloor, it is favorable if the scattering wave can be measured in the direction with this strong level. Therefore, to catch this strong scattering wave, an underwater vehicle was used. A hydrophone array was attached at the head of the underwater vehicle to accumulate over a series of pings. This report deals with the way and results of these measurements.

\section{2:45}

2pUW8. Buried target detection with a synthetic aperture sonar. John Piper (NSWC-PC, 110 Vernon Ave., Panama City, FL 32407)

During SAX04 the NSWC-PC dual-frequency synthetic aperture sonar collected data from a buried target field. Detections of a buried fluid-filled sphere from above critical angle averaged $34 \mathrm{~dB}$ and from below critical angle averaged $24 \mathrm{~dB}$ with a ripple field with predominant $42-\mathrm{cm}$ spacing. These results are compared to simulated PCSWAT results.

\section{3:00-3:15 Break}

\section{$3: 15$}

2pUW9. Using a dual-band synthetic aperture sonar for imaging various seafloor compositions. John S. Stroud, Daniel Brown, Jr., Daniel Cook, Jose Fernandez (NSWC-PC Code HS11, 110 Vernon Ave., Panama City, FL 32407), Kerry Commander (Office of Naval Res., Arlington, VA 22203), David Kolesar, and Tom Montgomery (Appl. Res. Lab, State College, PA 16804)

The Small Synthetic Aperture Minehunter (SSAM) is a synthetic aperture sonar mounted aboard a 12.75-in. REMUS600 unmanned underwater vehicle transmitting both low $(10-50 \mathrm{kHz})$ and high $(105-135 \mathrm{kHz})$ frequency bands. The sonar and the vehicle are highly integrated, which allows precise pulse timing, attitude correction, and object geolocation. Operating simultaneously in both bands produces two independent images of each scene. The upper band is capable of $2.5-$ by $2.5-\mathrm{cm}$ resolution that provides high-fidelity images of proud targets. The lower band, processed to 7.5 - by $7.5-\mathrm{cm}$ resolution, adds clutter reduction as well as capabilities against buried targets. The dual bands of this sonar have proved to be a robust solution for minehunting in the littorals. In the past 18 months, SSAM has been deployed nine times. In these tests, the system has operated against bottom types including mud, sand, rippled sand, layered mud and sand, posidonia, and coral. Imagery from sea tests showing all of these bottom types will be presented. [The authors would like to acknowledge support from the ONR, Code 32.]

2pUW10. Performance of a buried object scanning sonar against targets buried in sand at the SAX-04 (Sediment Acoustics Experiment 2004) site. Steven Schock (Dept. of Ocean Eng., Florida Atlantic Univ., 777 Glades Rd., Boca Raton, FL 33431, schock@oe.fau.edu)

The performance of synthetic aperture processing for imaging buried targets in the band of 3 to $20 \mathrm{kHz}$ is quantified by measuring the SNR of 3-D acoustic images of targets buried in sand at the SAX-04 site. A buried object scanning sonar consisting of an omnidirectional projector and a 40-channel hydrophone line array is towed within $10 \mathrm{~m}$ of the seabed at various horizontal offsets to buried targets to measure the SNR of target echoes as a function of grazing angle. Detections of the targets occurring at grazing angles smaller than the critical angle suggest that buried targets can be detected using the evanescent boundary wave thereby improving the range of the sonar for detecting shallow-buried targets. Acoustic images of buried targets also show that backscattering from sand ripples, sediment variability, and sediment layer interfaces limit the performance of the sonar for detecting and imaging buried objects.
$3: 45$

2pUW11. The design of a boundary element package for treating acoustic scattering from buried objects. Ralf Burgschweiger, Martin Ochmann (Univ. of Appl. Sci., Dept. of Mathematics, Phys., Chemistry, TFH Berlin Luxemburger Strasse 10, D-13353 Berlin, Germany), and Bodo Nolte (Federal Armed Forces Underwater Acoust. and Marine Geophys. Res. Inst. (FWG), D-24148 Kiel, Germany)

The target strength of objects placed in water and partially buried in the sediment are calculated by means of a boundary element solver. For this reason, a boundary element code with suitable pre- and postprocessor is developed. With the help of the preprocessor, the scattering objects within the transition range between fluid and sediment can be modeled. The preprocessor can manage large and complex meshes (more than 100000 elements) and is able to import NASTRAN and ANSYS meshfiles. It automatically generates closed geometric meshes. These meshes are used as database for the BEM calculations. For reducing computing time, the boundary element solver runs on a high number of personal computers in parallel equipped with Windows- or Linux-operating systems. These calculator hosts will receive their task from the preprocessor using TCP/IP-based interprocess communication. The parallel calculations can be performed as a service in the background and remotely administrated by the postprocessor. The postprocessor polls the results from the calculator hosts, delivers them to the appropriate projects, and provides visualization and analysis of the results. The efficiency of such a parallel processing boundary element package will be demonstrated for the acoustic scattering at simple test objects consisting of a high number of surface elements.

\section{4:00}

2pUW12. Subcritical grazing angle detection of targets buried under a rippled sand interface. Joseph L. Lopes, Carrie L. Dowdy, Raymond Lim (Naval Surface Warfare Ctr., Code HS11, 110 Vernon Ave., Panama City, FL 32444), Dajun Tang, Kevin L. Williams, and Eric I. Thorsos (Univ. of Washington, Seattle, WA 98105-6698)

Controlled acoustic measurements were conducted to investigate subcritical detection of targets buried in sand having a rippled sediment-water interface. The measurements were performed in a freshwater test-pond with a $1.5-\mathrm{m}$ layer of sand on the bottom. Bottom ripple was formed by scraping the sand with a machined rake. Broadband (10 to $50 \mathrm{kHz})$ transducers were placed onto an elevated rail that translated horizontally permitting data to be processed using synthetic aperture sonar (SAS) techniques. Targets included silicone oil-filled spheres and flat-endcapped, solid aluminum cylinders. Data were acquired for various ripple wavelengths, heights, and orientations. For each bottom configuration, the seabed roughness over the buried target was measured to verify the ripple parameters and to estimate the small-scale roughness spectrum. This information was used in models predicting backscatter signal levels from the target and bottom. Results for spherical targets have generally been good. However, when cylindrical targets were inspected, model agreement was variable for reasons not fully understood. Characteristics of the target return are found to depend sensitively on ripple height and wavelength, and bottom parameters. Results are discussed with an eye towards understanding previously unexplained field test detections of buried cylindrical targets. [Work supported by ONR and SERDP.]

\section{$4: 15$}

2pUW13. Scattering of evanescent waves incident on targets in a simulated sediment. Curtis F. Osterhoudt and Philip L. Marston (Dept. of Phys. and Astron., Washington State Univ., Pullman, WA 99163)

When a sound beam in water is incident on a sediment at a sufficiently small grazing angle, the resulting wave field in the sediment is complicated, even for the case of flat, fluidlike sediments. The wave field in the sediment for a sound beam from a simple transducer has an evanescent component and diffractive components. This situation has been experimentally simulated by using a combination of previously described immiscible liquids [Osterhoudt et al., J. Acoust. Soc. Am. 117, 2483 (2005)]. Measured wave-field features are similar to those seen in a wave-number- 
integration-based synthesis (using OASES) for a related problem. Targets are placed in the evanescent wave field to simulate scattering by targets buried in marine sediments. Features of the observed backscattering are relevant to detecting and clarifying objects in sediments. Measurements include the resonant excitation of the organ-pipe mode of open water-filled cylinders previously studied with free-field illumination [Osterhoudt and Marston, J. Acoust. Soc. Am. 113, 2334 (2003)]. The degradation of the backscattering with increasing target depth and with target tilt were measured and modeled. Simple approximate models of these properties are facilitated by the localized coupling processes for the excitation of (and radiation by) organ-pipe modes. [Work supported by ONR.]

\section{4:30}

2pUW14. Observations of subcritical acoustic penetration of a sandy seafloor. Darrell R. Jackson, Brian T. Hefner, Kevin L. Williams, and Eric I. Thorsos (Appl. Phys. Lab., Univ. of Washington, 1013 NE 40th St., Seattle, WA 98105-6698,drj@apl.washington.edu)

During the sediment acoustic experiments, SAX99 and SAX04, buried hydrophone arrays were used in conjunction with sources mounted on a movable tower to study subcritical acoustic penetration. The seafloor at the SAX99 site displayed prominent, regular sand ripples of 48-cm wavelength. These caused downward diffraction of sound incident below the critical angle, resulting in elevated sound-pressure fields in the sediment. These measurements were made over the frequency range $11-50 \mathrm{kHz}$ and were in substantial agreement with a first-order perturbation model. For $\mathrm{SAX} 04$, the frequency range was extended down to $2 \mathrm{kHz}$, but the site had weaker, more disorganized ripples. As expected, this resulted in weak acoustic penetration, with the pressure field primarily due to the evanescent wave. To further test the penetration model, artificial ripples of wavelength 50 and $75 \mathrm{~cm}$ were created by divers. These ripples increased sound penetration levels by $5-10 \mathrm{~dB}$ when the incidence azimuthal direction was normal to the ripple crests. The diffraction angles of the penetrating sound were found to be consistent with the predictions of the perturbation model. [Work supported by ONR.]

\section{$4: 45$}

2pUW15. Backscattering from sediments: Comparison of scattering strengths from a simple sand and from a sand/mud environment. Kevin Williams, Eric Thorsos, Dajun Tang, and Darrell Jackson (Appl. Phys. Lab., Univ. of Washington, 1013 NE 40th St., Seattle, WA 98105-6698,williams@apl.washington.edu)

Two sediment acoustics experiments were carried out within $1 \mathrm{~km}$ of each other off the coast of Florida, the first in 1999 and the second in 2004. Though close in space the sediment was very different due to the effects of Hurricane Ivan in 2004. Hurricane Ivan resulted in the deposition of mud patches on top of the medium sand. Subsequent weather events deposited sand on top of these patches. The backscattering at each site will be presented for frequencies from 20 to $1000 \mathrm{kHz}$ and grazing angles from 20 to $45 \mathrm{deg}$. At angles below the critical angle (30 deg) and frequencies below $150 \mathrm{kHz}$ the backscattering measured in the two experiments is consistent given the difference in the sediment roughness spectra.
Above the critical angle, however, the backscattering measured in the 2004 experiment is several dB higher than in the 1999 experiment and increases immediately above the critical angle instead of decreasing. This difference is attributed to an increase in sediment volume scattering in 2004. From 150 to $1000 \mathrm{kHz}$ the data from both years indicate a change in scattering mechanism below the critical angle to a mechanism differing from sediment interface roughness scattering. [Work supported by ONR.]

\section{5:00}

2pUW16. Measurements of interface roughness and examination of near bottom interface properties. Dajun Tang, Brian Hefner, Kevin Williams, and Eric Thorsos (Appl. Phys. Lab., Univ. of Washington, Seattle, WA 98105)

A series of measurements was made of sediment properties during SAX04 over a 40-day period using IMP2, a sediment conductivity system; a stereo camera system made fine-scale interface roughness measurements toward the end of this period. During this period, several weather events (including Hurricane Ivan and a subsequent tropical storm) altered the sediment properties. The large, nonsymmetric ripples resulting from the hurricane decayed to a more symmetric form over time, while mud suspended in the water column was found first on top of the sand and then trapped just beneath the interface. Comparison of the interface roughness power spectra from IMP2 and the stereo camera shows good agreement at overlapping spatial scales and the stereo measurements extend the IMP2 estimation of the power spectra to a much higher spatial frequency. Complementary cores taken by a team from the Naval Research Laboratory at the same sites where IMP2 was deployed confirmed the depth and location of the imbedded mud found by IMP2. The implications of these environmental measurements to acoustic backscattering will be discussed. [Work supported by the ONR.]

\section{$5: 15$}

2pUW17. Laboratory study of high-frequency scattering from watersaturated granular sediments. Anatoliy Ivakin (APL-UW, 1013 NE 40th St., Seattle WA 98105), Jean-Pierre Sessarego, and Regine Guillermin (CNRS/LMA, 13402 Marseille cedex 20, France)

Sound backscattering and reflection from water-saturated granular sediments at frequencies from $200 \mathrm{kHz}$ to $7 \mathrm{MHz}$ were studied in controlled laboratory conditions. Two kinds of well-sorted sandy sediments, fine and coarse sands, and two kinds of glass beads with corresponding sizes were chosen for the study. The two types of sand had narrow lognormal size distributions of particles with the mean diameters 0.25 and 1.5 $\mathrm{mm}$ for fine and coarse sand, respectively. The sediments were degassed and their surface was flattened carefully. In these conditions, the grainscattering mechanism can be considered as a dominating factor controlling incoherent component of the field scattered from the sediment. Frequency dependencies for the backscattering strength at various grazing angles and the reflection coefficient at normal incidence were measured. The effects related to the sediment grain size are analyzed and their possible applications to remote sensing of marine sediments are discussed. [Work supported by ONR and CNRS.] 